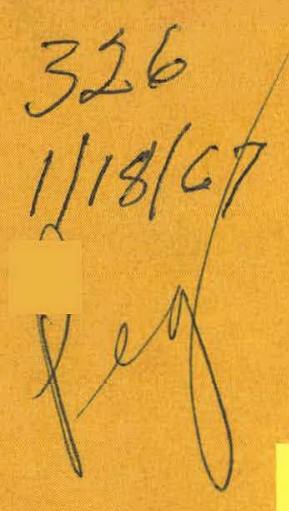

IN-1015

December 1966

MASTE

\title{
TABULATION OF THE
}

TOTAL. NEUTRON CROSS SECTION OF ${ }^{232} \mathrm{U}$

O. D. Simpson, M. S. Moore and J. R. Berreth

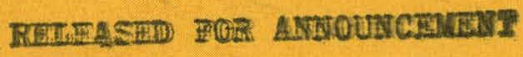

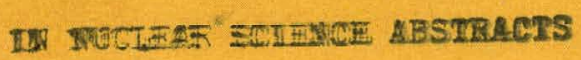

IDAHO NUCLEAR CORPORATION

NATIONAL REACTOR TESTING STATION

IDAHO FALLS, IDAHO 


\section{DISCLAIMER}

This report was prepared as an account of work sponsored by an agency of the United States Government. Neither the United States Government nor any agency Thereof, nor any of their employees, makes any warranty, express or implied, or assumes any legal liability or responsibility for the accuracy, completeness, or usefulness of any information, apparatus, product, or process disclosed, or represents that its use would not infringe privately owned rights. Reference herein to any specific commercial product, process, or service by trade name, trademark, manufacturer, or otherwise does not necessarily constitute or imply its endorsement, recommendation, or favoring by the United States Government or any agency thereof. The views and opinions of authors expressed herein do not necessarily state or reflect those of the United States Government or any agency thereof. 


\section{DISCLAIMER}

Portions of this document may be illegible in electronic image products. Images are produced from the best available original document. 
PRINTED IN USA. PRICE \$3.00. AVAILABLE FROM THE CLEARINGHOUSE FOR FEDERAL SCIENTIFIC AND TECHNICAL INFORMATION, NATIONAL BUREAU OF STANDARDS,

U. S. DEPARTMENT OF COMMERCE, SPRINGFIELD, VIRGINIA 22151

\section{LEGAL NOTICE}

This report was prepared as an account of Government sponsored work. Neither the United States, nor the Commission, nor any person acting on behalf of the Commission:

A. Makes any warranty or representation, express or implied, with respect to the accuracy, completeness, or usefulness. of the information contained in this report, or that the use of any information, apparatus, method, or process disclosed in this report may not infringe privately owned rights; or

B. Assumes any liabilities with respect to the use of, or for damages resulting from the use of any information, apparatus, method, or process disclosed in this report.

As used in the above, "person acting on behalf of the Commission" includes any employee or contractor of the Commission, or employee of such contractor, to the extent that such employee or contractor of the Commission, or employee of such contractor prepares, disseminates, or provides access to, any information pursuant to his employment or contract with the Commission, or his employment with such contractor. 


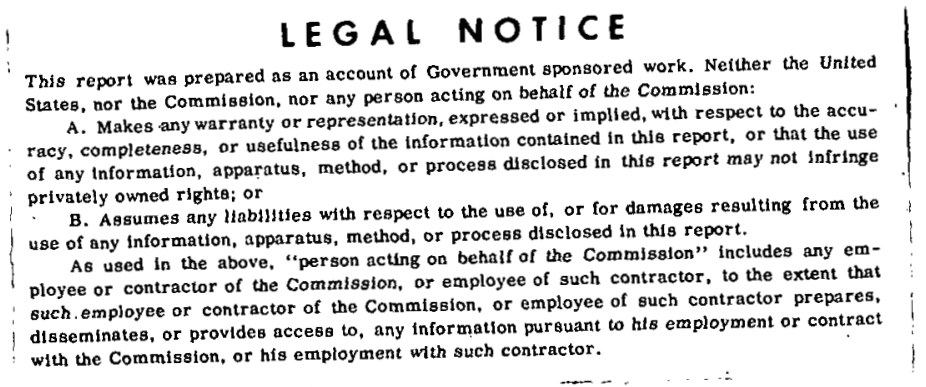

\section{LEGAL NOTICE}

an account of Government sponsored wor ing

B. Any information, apparatus, michod, or process disclose As used in the above, "person acting on behall of the Coh contrat to the extent tha such employee or contractor of the Commlssion. or employee of auch ch with the Commission, or his empioyment with such contractor.
IN -1015

Issued: December 1966

Physics

TID-4500

CFSTI PQICES

H.C. 3.00 , MN . 65

TABULATION OF THE TOTAL NEUTRON CROSS SECTION OF ${ }^{232} \mathrm{U}$

O. D. Simpson, M. S. Moore and J. R. Berreth

RELEASED FOR ANNOUNCEYENT

IN NUCLEAR SCIENCE AESTRACTS

IDAHO NUCLEAR CORPORATION

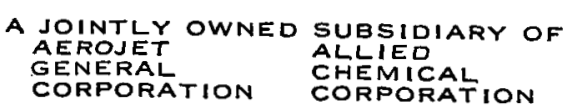
$\begin{array}{ll}\text { GENERAL } & \text { CHEMICAL } \\ \text { CORPORATION CORPORATION }\end{array}$

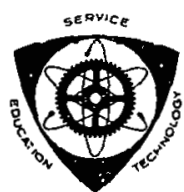

U. S. Atomic Energy Commission Research and Development Report Issucd Under Contraot $\Lambda \mathrm{T}(10-1)-1230$ Idaho Operations Office 
THIS PAGE

\section{WAS INTENTIONALLY LEFT BLANK}


Tabulation of the Total Neutron Cross Section of ${ }^{232} \mathrm{U}$

O. D. Simpson, M. S. Moore ${ }^{\ddagger}$ and J.R. Berreth

\section{Abstract}

The total neutron cross section of ${ }^{232} U$ has been measured from 0.01 to $10,000 \mathrm{eV}$ using the Materials Testing Reactor (MTR) fast chopper. A $2200 \mathrm{~meter} / \mathrm{sec}$ total neutron cross section of $165 \pm 10 \mathrm{barms}$ was determined. Multilevel parameters are listed for resonances below $30 \mathrm{eV}$. Results of the analysis indicate that two fission channels are needed to describe the experimental data. A tabulation of the data is given in the attached appendix.

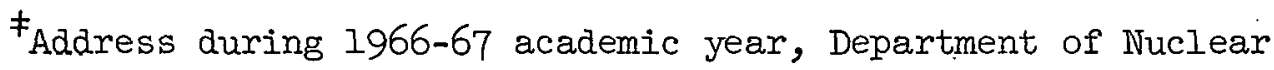
Engineering and Science, Rensselaer Polytechnic Institute, Troy, New York. 
Table of Contents

Abstract. . . . . . . . . . . . . . . . . . $\frac{\text { Page }}{\text { No }}$

I. Introduction. . . . . . . . . . . . . . . . : I

II. Experimental Procedure. . . . . . . . . . . . 2

III. Analysis. . . . . . . . . . . . . . . . . 7

IV. Discussion. . . . . . . . . . . . . . . . . . . 9

V. Acknowledgments . . . . . . . . . . . . . . . 1.1

References. . . . . . . . . . . . . . . . . . 13

List of Tables

Table I - Measured Total Neutron Cross Section of ${ }^{232}$ U. . . $16^{\circ}$

Table II - Calculated Neutron Cross Sections fur 232 U. . . . 17

Table III - ${ }^{232}$ U Neutron Resonance Parameters for a Multilevel Two-Channel Fit ............. 18

Table IV - ${ }^{232}$ U Neutron Resonance Parameters for a Multilevel One-Channel Fit................. 19

List of Figures

Figure 1 - Total Neutron Cross Section From 0.01 to $1.0 \mathrm{eV}$. . 20

Figure 2 - Total Neutron Cross Section From 1 to 10,000 eV. •. 21

Figure 3 - The Difference in Cross Section Between Two ${ }^{23}$ U
Samples. . . . . . . . . . . . . 22

Appendices

Appendix A - Tabulation of the Total Neutron Cross Section For Different, $232_{\mathrm{T}}$ Samplea . . . . . . . .

Appendix B - Tabulation of the Total Neutron Cross Section of ${ }^{232} \mathrm{U}$ from 0.01 to $10,000 \mathrm{eV}$......... 
Tabulation of the Total Neutron Cross Section of ${ }^{232} \mathrm{U}$

O. D. Simpson, M. S. Moore and J. R. Berreth

\section{Introduction}

The low-energy neutron cross sections of ${ }^{232} \mathrm{U}$ are of interest for several reasons. In the ${ }^{232} \mathrm{Th}-{ }^{233} \mathrm{U}$ breeding cycle, ${ }^{232} \mathrm{U}$ is formed in relatively low concentrations (approximately 50-100 ppm of the uranium produced), primarily as a result of the reaction ${ }^{232}$ Th $(n, 2 n){ }^{23 I_{T h}}$ followed by beta decay to ${ }^{231} \mathrm{~Pa}$ and then by slow neutron capture. The presence of ${ }^{232} U$ in reactor-produced ${ }^{23} \mathrm{U}$ may cause a serious fabrication and handling hazärd for ${ }^{233} \mathrm{U}$-fueled reactors. This is because ${ }^{232} \mathrm{U}$ decays with a 72 year half-life to 1.9 year ${ }^{228}$ Th, the parent of a shortlived decay chain ending in ${ }^{208} \mathrm{~Pb}$ with the emission of a highly penetrating $2.62 \mathrm{MeV}$ gamma ray. The existence of small quantities of ${ }^{232} \mathrm{U}$ in ${ }^{233_{U}}$ fuel requires that remote facilities be used when fabricating and haruling such fuel. The cross sections of ${ }^{2.32} \mathrm{U}$ are of interest in predicting ${ }^{232} \mathrm{U}$ burn-out relative to that of ${ }^{233} \mathrm{U}$ and the radiation hazards which may be involved in handling recycled $233_{U}$ reactor fuel. The fact that there are no reactors in existence at present which use $233_{U}$ as fuel is probably due in part to the presence of ${ }^{232} \mathrm{U}$ in such fuel.

The cross sections of ${ }^{232} \mathrm{U}$ are also of physical interest. The most commnn fissilc nuclides, ${ }^{23} 3_{\mathrm{U}},{ }^{235} \mathrm{U},{ }^{239} \mathrm{Pu}$ and ${ }^{241} \mathrm{Pu}$ are even-odd isotopes, and the low-energy neutron resonance structure is very difficult to analyze because it is so complex. The compound nucleus formed by adding a slow neutron to one of these common fissile nuclides is eveneven, and there are two possible spin states. The resonances are closely 
spaced, and strong interference is observed in the fission component. It is almost impossible to obtain a meaningful analysis of such data without some a priori information about the resonance spins. In order to further the understanding of the fission process, one should also investigate the resonance behavior of an even-even fissile target nuclide such as ${ }^{232} \mathrm{U}$. Here, since the initial target spin is $0^{+}$, only one compound nucleus spin state is formed, $1 / 2^{+}$. The resonances arc widely spaced, and it is of interest to know what kind of interference effects, if any, can be observed in the fission of such an even-odd compound nucleus.

\section{Experimental Procedure}

The ${ }^{232} \mathrm{U}$ used in these measurements consisted of approximately $1 \mathrm{~g}$ of material obtained from Oak Ridge National Laboratory (ORNL) in the form of uranium oxide, $\mathrm{U}_{3} \mathrm{O}_{8}$. It had been prepared by reactor irradiation of ${ }^{231} \mathrm{~Pa}$ and subsequent separation of the ${ }^{232} \mathrm{U}$ produced. The isotopic composition of the material was as follows: $99.24 \%{ }^{232} \mathrm{U}, 0.7 \mathrm{~m} 233_{\mathrm{U}}$, with traces of ${ }^{235} \mathrm{U}$ and ${ }^{238} \mathrm{U}$.

From this material, samples were prepared for transmission measurements, which were made with the Materials Testing Reactor (MTR) fast chopper and associated radioactive-sample changer. (I). The prucedure used in preparing uniform samples, by mixing weighed quantilles of sample material with aluminum powder and pressing the mixture into a solid compact, has been described in detail ${ }^{(2)}$. Three samples of ${ }^{232} \mathrm{U}_{3} \mathrm{O}_{8}$ in Al were prepared in this way, having inverse thicknesses of 2205 , 576.9 and 556.6 barms/atom of ${ }^{232} \mathrm{U}$ at the time of preparation. During one phase of the experiment, the two thicker samples were combined to 
form a sample having an inverse thickness of 283.2 barns/atom. The thinnest sample was prepared from the $\mathrm{U}_{3} \mathrm{O}_{8}$ as it was received from ORNL; however, the two thicker samples were reprocessed before packaging to remove the ${ }^{228}$ Th and other daughter products. This was carried out as follows: The $\mathrm{U}_{3} \mathrm{O}_{8}$ was dissolved in $8 \mathrm{~N} \mathrm{HCl}$ with a trace of $\mathrm{HNO}_{3}$. This uranium solution was then loaded onto a Dowex 1-X8 column. The 228 Th was washed through the column with succeeding washes of $8 \mathrm{~N} \mathrm{HCl}$. The uranium was then eluted with a water wash, precipitated, filtered, dried and ignited to the oxide at $800^{\circ} \mathrm{C}$.

Details of the experimental procedure have been discussed elsewhere ${ }^{(3)}$, and will only be summarized here as follows: The ${ }^{232} \mathrm{U}$ sample was placed in the shielded sample changer at the point where the neutron beam enters the chopper rotor. The sample and open blank were cycled alternately into the beam at 10 -minute intervals, as were ${ }^{10} \mathrm{~B}$ filters and beam-blocking devices to measure backgrounds ${ }^{(4)}$. The times of flight of the neutrons over a 20 meter flight path were measured with a 4096channel analyzer.

Data were collected at intervals of a few weeks to a few months, over a period of two years, in order to observe the effects of radioactive decay of ${ }^{232} \mathrm{U}$ and buildup of daughter products. The total neutron cross section of ${ }^{232} \mathrm{U}$, as determined from the transmission data, is shown in Figs. 1 and 2. A tabulation of the data are given in Appendices $A$ and $B$.

In all the measurements taken on the ${ }^{23 \varepsilon} \mathrm{U}$ samples, there was observed an anomalous cross-section contribution, which raised the offresonance cross section from the expected 10-15 barns to between 30 and 54 barns, depending on the sample. Tffccts of this nature have been 
noted previously when making transmission measurements on highly radioactive samples; in particular, very much the same effect was observed for ${ }^{238} \mathrm{Pu}$ and ${ }^{182} \mathrm{Ta}$ samples $(5,6)$.

A correction for this anomalous cross section was carried out, under the assumption that it was due to a contaminant. One sample had a v.a.1. Iey crows sectiun of 54 barns and another, a valley cross section of 36 barns. It was assumed that the difference in these two cross sections gives the energy dependence of this contaminant. The energy dependencc of this cross section is shown in Fig. 3. The results indicate that the anomalous cross section is due primarily to a hydrogenous substance. (It can only be conjectured what the source of this contaminant might be: perhaps water or a hydrocarbon depositing on the sample f'rom radiation destruction of the neoprene o-ring gasket.) The shape of the contaminant curve was assumed to be given by this difference, and to be the same for all samples. Before the correction could be made, however, it was necessary to know how much of the contaminant was present in earh of the samples.

We chose to make the correction by normalizing the contaminant cross section as shown in Fig. 3 for each of the different samples to give total cross-section values for ${ }^{232} \mathrm{U}$ which were self-consistent over the region of energies covered. Three representative energy regions were considered: thermal (near $0.025 \mathrm{eV}$ ), the resulved resonance region (a few ev), and the unresolved resonance region (above $1 \mathrm{keV}$ ).

(1) In the thermal energy region, Halperin et al (7) found the radiative capture cross section of ${ }^{232} \mathrm{U}$ to be $78 \pm 4$ barns. Elson et al ${ }^{(8)}$ measured the fission cross section to be $81 \pm 15$ barns. The present data can be used as evidence that the correction for a non $1 / \mathrm{v}$ behavior 
of the absorption cross section in the region near $0.025 \mathrm{eV}$ does not change these numbers significantly. It was assumed that the absorption cross section of ${ }^{232} \mathrm{U}$ at $0.0253 \mathrm{eV}$ is given by $159 \pm 16$ barns, the sum of these two components. A scattering cross section at $0.0253 \mathrm{eV}$ of $14 \pm 5$ barns was estimated by using the two channel multilevel parameters (to be described in Section III below) and by assuming a potential scattering cross section of $15 \pm 5$ barns. The expected scattering cross section is lower than the potential scattering cross section because of the destructive interference of potential scattering with resonance scattering of the $5.9 \mathrm{eV}$ resonance.

(2) In the region of $1-3 \mathrm{eV}$, there is a valley in the cross section of ${ }^{232} \mathrm{U}$. An analysis of the observed resonance structure of ${ }^{232} \mathrm{U}$ (Section III) gave a value of $4 \pm 2$ barns for the absorption cross section in this valley, and a value of $12.5 \pm 5.0$ barns for the scattering cross section.

(3) At neutron energies above $1 \mathrm{keV}$ the resonances can no longer be observed as such, because uf resolution broadening. From nur.tear systematics, a value of $1.0 \pm 0.2 \times 10^{-4}$ can be expected for the neutron strength function $\left\langle\Gamma_{n}^{O} / D>(9)\right.$. If one assumes a level spacing at $I \mathrm{keV}$ to be the same as at lower energies, one can calculate an average resonance cross section of $13 \pm 3$ barns.

A linearized least-squares program (10) was used to estimate how much hydrogenous contaminant was present in each of the samples. It was assumed that the measured total cross section $\left(\sigma_{n T}\right)_{i j}$ for the $i$ th sample and for the $j$ th ncutron energy region could be written as 


$$
\left(\sigma_{n T}\right)_{i j}=\left(\sigma_{n X}\right)_{j}+\left(\sigma_{n n}\right)_{j}+k_{i}\left(\sigma_{n c}\right)_{j},
$$

where $\left(\sigma_{n X}\right)_{j}$ is the absorption cross section of ${ }^{232} U$ in the energy region of interest, $\left(\sigma_{n n}\right)_{j}$ is the scattering cross section, $\left(\sigma_{n c}\right)_{j}$ is the hydrogenous contaminant cross section, and $k_{i}$ is the amount of contaminant relative to ${ }^{232} \mathrm{U}$ for the $i$ th sample. In order to reduce the number of variahles, it was accumcd that the enlergy variation of $\left(\sigma_{n c}\right)_{j}$ was that given by the derived contaminant cross' section shown in Fig. 3. Equation 1 was linearized and used, with auxiliary equations based on the values consistent with (1) integral measurements at thermal energies, (2) multilevel results in the low recononce region, and (3) strength function systematics at $1 \mathrm{keV}$, to determine the best contaminant correction (that which gives the least square error) for each run. The measured cross section, values for the three regions discussed above are listed in Table I. The input parameters and results of the final iteration of the least-squares determination are given in Table II.

Some discussion may be required to justify the assumption that the off-resonance cross section is due to a contaminant. The possibility exists, of course, that the off-resonance cross section of ${ }^{232} U$ actually amounts to perhaps 50 barns, instead of the 10-15 barñs common to other heavy nuclides $(11)$. James ${ }^{(12)}$ has reported the off-resonance fission cross section of ${ }^{232} \mathrm{U}$ as $25-30$ barns in the $10 \mathrm{w}$ eV region. One can interpret this as evidence that the anomalous off-resonance cross section belongs to ${ }^{232} \mathrm{U}$, and not to an unknown contaminant. The present data do not support such an interpretation, however, for two reasons: 
(1) The off-resonance cross section is constant rather than showing the $1 / \mathrm{v}$ neutron energy dependence which is required if it is due to fission.

(2) The magnitude of the off-resonance component is not stoichiometrically related to the amount of ${ }^{232} \mathrm{U}$ in the samples for the various runs.

\section{Analysis}

In the initial reporting of these measurements (13), in June of 1963, it was concluded from very preliminary data that ${ }^{232} \mathrm{U}$ resonances do show a substantial fission component, since the resonances are much wider than any observed heavy-element capture resonances. Later, direct measurements of the fission cross section by G. D. James (12) at Harwell confirmed these results. The resonances in ${ }^{232} \mathrm{U}$ are observed to be .. relatively widely-spaced, so that interference effects in fission would not be expected to be very pronounced. In the earliest measurements (13), talken with the thinnest sample, it was found that the data were consistent with a Breit-Wigner single-level description. Only in the thicker sample transmission data are the effects large enough to demonstrate the existence of interference. The transmission data were analyzed by a multilevel formula which treats interference among fission resonances using one or two fission channels. The solid curve in Fig. 2 shows the results of the multilevel two-channel analysis, the parameters of which are listed in Table III. In analyzing the data, it was found that at least two fission channels are required for the five resonances considered. A single-fission-channel description was found to be inadequate, particularly lu lhe region of 18 to $23 \mathrm{eV}$. All possihle combinations 
of signs on the interference terms were used in attempting to fit the data with one fission channel; the dashed line below $31 \mathrm{eV}$ shows the best of the single-channel descriptions, and the resonance parameters for which are given in Table IV. Shown also are the resonance parameters reported by James ${ }^{(12)}$, from the fission cross-section measurements. This one-channel fit to the data was obtained by allowing the resonances to interfere in the manner recommended by James in his analysis of the fission cross section of ${ }^{232} \mathrm{U}$. However, James' data are necessarily thin sample data, which do not have the sensitivity (because of counting statistics) in discerning small changes in the magnitude of the cross section between resonances. Hence from the fission data alone one could probably not tell that a single-fission-channel description is inadequate. James' parameters are not in agreement with the present analysis, primarily because of the values of $\Gamma_{n}^{0}$. The cross section calculated from James' parameters agree better with the present data if the values of $\Gamma_{\mathrm{n}}^{\circ}$ are increased by approximately $50 \%$. This is equivalent to attributing the discrepancy to an error in determining the amount of fissile material used in the Harwell measurements. It is difficult to see how possible errors in the present experiment can account for such a discrepancy.

A previous section of this paper described the experimental measurement, in which there was a large correction required to forse self-consistency in the off-resonance cross section. But in the analysis, it is just the off-resonance cross section which is most sensitive to multilevel interference effects. It is thus natural to question the validity of an analysis which appears to depend completely on a correction which was in turn forced to agree with the analysis. 
In actuality, the magnitude of the correction has virtually no effect on the results of the analysis above $1 \mathrm{eV}$. The interference in fission between two resonances does not depend on the magnitude of the offresonance cross section. For example, in Fig. 2, it is apparent that the 12.7 and $21.1 \mathrm{eV}$ resonances interfere constructively, and shifting the baseline by a constant will not change the interference appreciably. Shifting the baseline is equivalent to making a constant correction, and the correction for a hydrogenous contaminant is constant from 1 to $30 \mathrm{eV}$.

\section{Discussion}

The conclusion reached in the analysis, that a single-fission channel is not adequate to describe the data, is somewhat surprising. only one channel is expected for s-wave neutron induced fission of ${ }^{232}{ }^{\prime \prime}$, and this is presumably the same channel observed by Lamphere $(14,15)$ in recent studies of the fast neutron fission of ${ }^{23^{\prime}} \mathrm{H}$ and other ever-even : targets. Une expects the fission channel sequence for $\left({ }^{232} U+n\right)$ to be the same as for other even-even target nuclides $\left({ }^{230}\right.$ Th $\left.+n\right),\left({ }^{232}\right.$ Th $\left.+n\right)$ and $\left({ }^{234} U+n\right)$. The channel sequence postulated for fast neutron fission is $1 / 2^{+}, 3 / 2^{-}, 1 / 2^{-}$. These channels are found to dominate the fast neutron-induced fission cruss section for several hundred keV above the threshold, and there is no need to assume the existence of a second $1 / 2^{+}$ channe1. On the basis of systematics of neutron-induced fission threshholds of the even-even isotopes of uranium, one cannot really predict with any certainty whether ${ }^{232} \mathrm{U}$ ought to be a fissile nuclide. Direct measurements of the low-energy neutron fission cross sections $(8,12)$ show that it is. But certainly the threshold fus the $1 / 2^{+}$fission rhanne?. 
does not lie very far below the neutron binding energy. One can make estimates about the possible existence of a second $1 / 2^{+}$channel lying within a few hundred keV of the lowest threshold, such as that in the work of Lamphere ${ }^{(14)}$ and others, the two channels might appear as one. Following Nilsson $(16)$, Vandenbosch ${ }^{(17)}$ has made calculations of expected single particle neutron levels which could correspond to the observed channel structure. A second low-lying $1 / 2^{+}$channel is not expected for any reasonable deformation at the sadule point.

Nevertheless, the present data are not consistent with a single fission channel for the observed levels, and these levels are far too strong to be anything but $s$-wave resonances. It is of interest to note that the wide resonances do show the expectcd interference; on $\perp$ y the two narrowest levels are not consistent with the single-fission-charnel interpretation. This may indicate an effect recently suggested by Lymn (18). Noting that there appears to be a lack of very small fission widths in all fissile nuclide resonances, Lynn suggested that a directfission process $(n, \gamma f)$ may be responsible. This process is analogous to the direct capture of slow neutrons, discussed by Lane and Lynn $(19,20)$, where the final state, assumed to lie above its fission threshold, could then undergo fission. The probability of such a process has been estimated by Lynn for the common fissile nuclides (18). An upper limit for the fission width is a few $(4 \mathrm{nr} 5) \mathrm{mV}$. Such a proccss might be invoked to account for the lack of interference in the narrowest ${ }^{232} \mathrm{U}$ resonances. In order for the $(n, \gamma f)$ reaction to be of importance in ${ }^{232} \mathrm{U}$ resonances, the channel sequence must be different from $1 / 2^{+}, 3 / 2^{-}$, $1 / 2^{-}$. The $1 / 2^{+}$channel must lie high enough above the $3 / 2^{-}$or $1 / 2^{-}$ channel, or both, to permit fission to occur with a reasonable probability 
after energy Ioss by gamma emission. The fast neutron angular distribution data ${ }^{(14)}$, not being sensitive to parity, would be equally consistent with a channel sequence of $1 / 2^{-}, 3 / 2^{-}, 1 / 2^{+}$. A channel sequence with a negative parity band lying below a $1 / 2^{+}$band has been suggested by Rae ${ }^{(2 l)}$ as being more nearly consistent with the anomalously small resonance fission component of ${ }^{238} \mathrm{Pu}(22)$. Vorotnikov et al ${ }^{(23)}$ suggest the same kind of channel sequence as a result of studies of the fission threshold of ${ }^{238} \mathrm{Pu}$. Since ${ }^{232} \mathrm{U}$ should show the same fission channel. sequence as all other even-even nuclides, the $(n, \gamma f)$ reaction appears to be a likely reaction in ${ }^{232} \mathrm{U}$.

It has always been assumed in the multilevel analyses which have been carried out on the common fissile nuclides that the lack of knowledge of the resonance spin prevented one from determining the width parameters uniquely. It was expected that the present analysis, in which all resonances are characteristic of spin $1 / 2^{+}$, would be an especially simple case. This has not proved to be so; the simplicity has puinted instcad to another anomaly, the necessity of using morc than one fission channel in the description of the resonances. The mechanism of this anomaly remains to be established. The present analysis concerns only five resonances. One expects that future investigat1ons, involving hundreds of resonances and complete experiments in which both fission and capture are determined independently, will provide more definitive answers to the problems in low-energy neutron fission of ${ }^{232} \mathrm{u}$.

\section{Acknowledgment $\varepsilon$}

The authors would l1ke lu exprosi their appreriation to D. R. Staples 
and D. B. Hansen for their maintenance of the fast chopper equipment and to Mrs. A. L. Dittmer for assistance with the data processing. Thanks are due to Dr. C. D. Bowman and Dr. G. F. Auchampaugh for communicating some of the results of their own measurements of the fission cross sections of ${ }^{232} \mathrm{U}$ and ${ }^{238} \mathrm{Pu}$, prior to publication. Finally, it is a pleasure to acknowledge the many communications and helpful discussions with Dr. J. E. Lynn and Dr. G. D. James of the Atomic Energy Research Establishment. at Harwcl.1. 
References

1. F. B. SIMPSON and R. P. SCHUMAN, "Cross Section Measurements on Radioactive Samples" in "Neutron Time-of-Flight Methods," (J. Spaepen, ed.) p. 85. Euratom, Brussels (1961).

2. J. R. BERRETH, "Fabrication of Radioactive Samples for Cross-Section Measurements," Nuclear Applications 1, 230 (1965).

3. R. G. FLUHARTY, F. B. SIMPSON and o. D. SIMPSON, "Neutron Resonance Measurement of $\mathrm{Ag}, \mathrm{Ta}$ and ${ }^{238} \mathrm{U}$, "Phys. Rev. 103, 1778 (1956)

4. O. D. SIMPSON, R. G. FLUHARTY, M. S. MOORE, N. H. MARSHALL, F. B. SIMPSON, G. E. STOKES, T. WATANABE and T. E. YOUNG, "The Determination of Backgrounds for Neutron Time-of-Flight Spectrometer," Nuc. Inst. Methods 30, 293 (1964)

5. T. E. YOUNG, F. B. SIMPSON and M. S. COOPS, "Total Neutron Cross Section of ${ }^{238} \mathrm{Pu}, "$ Bull. Am. Phys. Soc. I, 305 (1962).

6. G. E. STOKES, unpublished data (1966).

7. J. HALPERIN, C. R. BALDOCK and J. H. OLIVER, "The Thermal Capture Cross Section and Resonance Integral of ${ }^{232} \mathrm{U}$," Nuc. Sci. and Engr. 21, 257 (1965).

8. R. ELSON, W. BENTLEY, A. GHIORSO and Q. VAN WINKLE, "The Thermal Neutron Fission and Capture Cross Section of ${ }^{232} \mathrm{U}$," Phys. Rev. 89 , 320 (1953): Authors reported $\sigma_{f}=83 \pm 15 \mathrm{~b}$, assuming ${ }^{232} \mathrm{U} \mathrm{T}_{\frac{1}{2}}=70 \mathrm{yrs}$. A corrected value of $81 \pm 15$ b was obtalned for $\frac{T_{1}}{2}=72$ yrs.

9. R. E. COTE', R. F. BARNS and H. DIAMOND, "Total Neutron Cross Section of ${ }^{2 ! 1 /} \mathrm{Cm}$, Phys. Rev. 1.34, Bl281 (1964).

10. J. R. SMITH, S. D. REEDER and R. G. FLUHARTY, "Measurement of the Absolute Value of Eta for ${ }^{233} \mathrm{U},{ }^{235} \mathrm{U}$ and ${ }^{239} \mathrm{Pu}$, using Monochromatic 
Neutrons," Bull. Am. Phys. Soc. 10, 1099 (1965), and USAEC Report ID0-17083 (1966).

11. See, for example, K. K. SETH, "Nuclear Radii by Low Energy Neutron Scattering," Revs. Mod. Phys. 30, 442 (1958).

12. G. D. JAMES, "The Fission Cross Section of ${ }^{232} \mathrm{U}$ from $4 \mathrm{eV}$ to $400 \mathrm{eV}, "$ Nuc. Phys. 55, 517 (1964).

13. J. R. BERRETH, M. S. MOORE and O. D. SIMPSON, "Total Neutron Cross Section of ${ }^{232} \mathrm{U}$," Trans. Am. Nuc. Soc. 6, 44 (1963).

14. R. W. IAMPHERE, "Fission Cross Section and Fragment Angular Distribution for Fast Neutron-Induced Fission of ${ }^{234} \mathrm{U}$, "Nuc. Phys. 38, 561 (1962).

15. R. W. LAMPHERE, "Channel Analyses of Neutron-Induced Fission of Even-Even Isotopes Near Threshold," Physics and Chemistry of Fission, Vol. I, p. 63, IAEA, Vienna (1965).

16. S. G. NILSSON, "Bending States of Individual Nucleons in Strongly Deformed Nuclei," Kgl. Danske Videnskab., Selskab, Mat-Pys. Medd. 29, No. $16(1.955)$.

17. R. VAINDENBOSCH, J. P. UNIK, J. R. HUIZENGA, "Fission-Fragment Angular, Energy, and Mass Division Correlations for the ${ }^{234} \mathrm{U}$ (d,pf) Reaction," Physics and Chemistry of Fission, Vol. I, p. 547, IAFA, Vienna (1965).

18. T. E. THYNN, "On the Slow Neutron, Gamma-Fission Reaction," Physics Letters 18, 31 (1965).

19. A. M. LANE and J. E. LYNN, "Theory of Radiative Capture in the Resonance Region," Nuc. Phys. 17, 563 (1960).

20. A. M. LANE and J. E. LYNN, "Anomalous Radiative Capture in the 
Neutron Resonance Region: Analysis of the Experimental Data on Electric Dipole Transitions," Nuc. Phys. 17, 586 (1960).

21. E. R. RAE, "Cross-Sections for Low-Energy Neutron-Induced Fission," Physics and Chemistry of Fission, Vol. I, p. 187, IAEA, Vienna (1965).

22. G. D. JAMES, "Fission Cross-Section Measurements on ${ }^{239} \mathrm{Pu},{ }^{241} \mathrm{Pu}$, ${ }^{232} \mathrm{U}$ and a Search for Fission components in ${ }^{238} \mathrm{Pu}$ Resonances," Physics and Chemistry of Fission, Vol. I, p. 235, IAEA, Vienna (1965).

23. P. E. VOROTNIKOV, S. M. DUBROVINA, G. A. OTROSHENKO and V. A. SHIGIN, "Channel Analysis of ${ }^{238}$ Pu Neutron Fission," Soviet Journal of Nuclear Phys. 3, 479 (1966). 
TABLE I

Measured Total Neutron Cross Section of ${ }^{232} \mathrm{U}$

Total Cross Sections

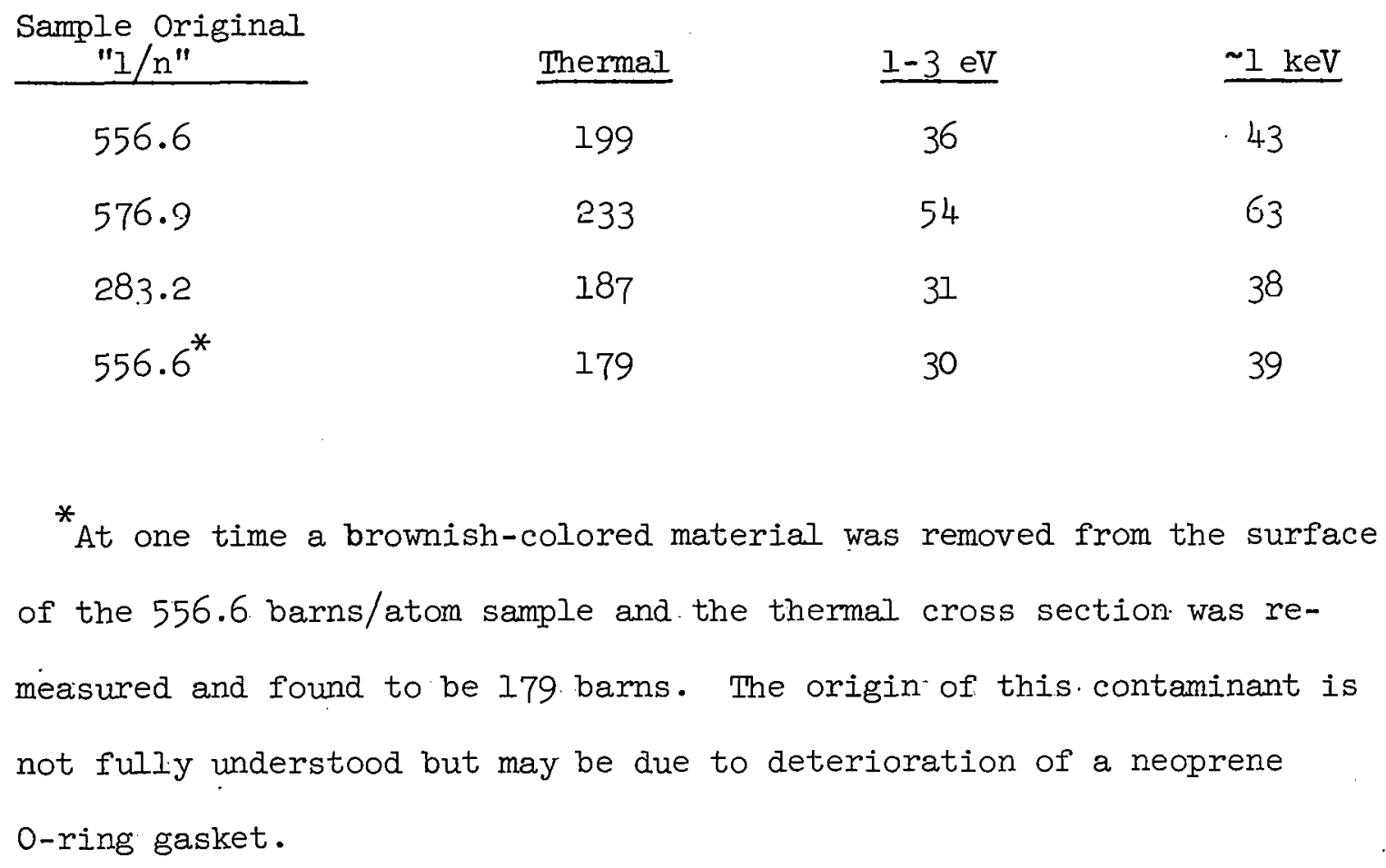


TABLE II

Calculated Neutron Cross Sections for ${ }^{232} \mathrm{U}$

Thermal

$\underline{1-3 \mathrm{eV}}$

$\sim \mathrm{l} \mathrm{keV}$

Input Output

Input Output

Data

Parameters Data

Parameters

$\sigma_{n X} \overline{159 \pm 16^{(a)}} 150.0 \pm 3.9$

$4 \pm 2^{(b)}$

$4.3 \pm 0.9$

Input

Output

$\sigma_{n n} 14 \pm 5^{(b)} 13.1 \pm 2.2$

$12 \cdot 5 \pm 5^{(b)}$

$14.6 \pm 1.715 \pm 5^{(d)}$

$13 \pm 3^{(c)}$
$15 \pm 5^{(d)}$

Data

$\sigma_{\mathrm{nT}} 173 \pm 17 \quad 163 \pm 10^{(\mathrm{e})} 16.5 \pm 6$

$18.9 \pm 1.928 \pm 6$

$12.9 \pm 1.3$

$14.7 \pm 1.8$

(20)

(n)

The input parameter data of this table were combined with the measured cross sections of Table I using a least-square routine to produce the output data listed above.

(a) Obtained from capture and fission data references 7 and 8 .

(b) Estimated from multilevel parameters Table III and assuming a potential scattering cross section of $15 \pm 5$ barns.

(c) Estimated from the neutron strength function $\Gamma_{n}^{0} / D=(1.0 \pm 0.2) \times 10^{-4}$

(d) Assumed value for the potential scattering cross section.

(e) The error was increased because of sample uniformity and thickness uncertainties. 
TABLE III

${ }^{232^{2}}$ U Neutron Resonance Parameters for a Multilevel Two-Channel Fit

$\begin{array}{llccc}E_{\mathrm{O}}(\mathrm{eV}) & \frac{\Gamma_{\mathrm{n}}^{\mathrm{O}}(\mathrm{mV})}{0.108} & \frac{\Gamma_{\mathrm{fl}}(\mathrm{mV})}{(\mathrm{a})} & \frac{\Gamma_{\mathrm{f2}}(\mathrm{mV})}{(\mathrm{a})} & \Gamma_{\gamma^{(\mathrm{mV})}}^{(\mathrm{b})} \\ -0.600 & 0.60 & -47 & & 47 \\ 5.985 & 1.81 & 24 & 263 & 47 \\ 12.73 & 0.39 & & -500 & 47 \\ 21.10 & 1.00 & 80 & & 47 \\ 23.87 & 0.45 & & -150 & 47 \\ 27.75 & & & & 47\end{array}$

Since the resonance parameters obtained from a multilevel analysis are not unique, it is impossible to assign errors to the resonance parameters. The quantity $\Gamma_{f l}$ and $\Gamma_{f 2}$ are the fission widths associated with the two fission channels.

(a) Fission widths are always positive; the sign is used to define the type of interference. Resonances having fission widths of the same sign interfere destructively between resonances while resonances with opposite signs interfere constructively.

(b) As sumed value. 
TABLE IV

${ }^{232}$ U Neutron Resonance Parameter for a Multilevel One-Channel Fit

\begin{tabular}{|c|c|c|c|c|c|c|c|}
\hline \multicolumn{2}{|c|}{$E_{0}(e V)$} & \multicolumn{2}{|c|}{$\Gamma_{n}^{O}(\mathrm{mV})$} & \multicolumn{2}{|c|}{$\Gamma_{f}(m V)(a)$} & \multicolumn{2}{|c|}{$\Gamma_{y}(\mathrm{mV})^{(\mathrm{b})}$} \\
\hline MTR & Harwell (c) & MTR & Harwell & MTR & Harwell & MTR & Harwell \\
\hline-0.600 & & 0.108 & & -47 & & 47 & \\
\hline 5.985 & 5.99 & 0.60 & $\cdot 32$ & 24 & $31 \pm 24$ & 47 & 50 \\
\hline 12.73 & 12.75 & 1.81 & 1.23 & 263 & $261 \pm 25$ & 47 & 50 \\
\hline 21.10 & 21.04 & 0.39 & $\cdot 35$ & -500 & $-913 \pm 150$ & 47 & 50 \\
\hline 23.87 & 23.86 & 1.00 & .68 & 80 & $80 \pm 40$ & 47 & 50 \\
\hline 27.75 & 27.7 & 0.45 & .42 & -150 & $-540 \pm 40$ & 47 & 50 \\
\hline
\end{tabular}

(a) Fission widths are always positive; the sign is used to define the type of interference. Resonances having fission widths of the same sign interfere destructively between resonances while resonances with opposite signs interfere constructively.

(b) Assumed values.

(c) Reference 12 this report. 


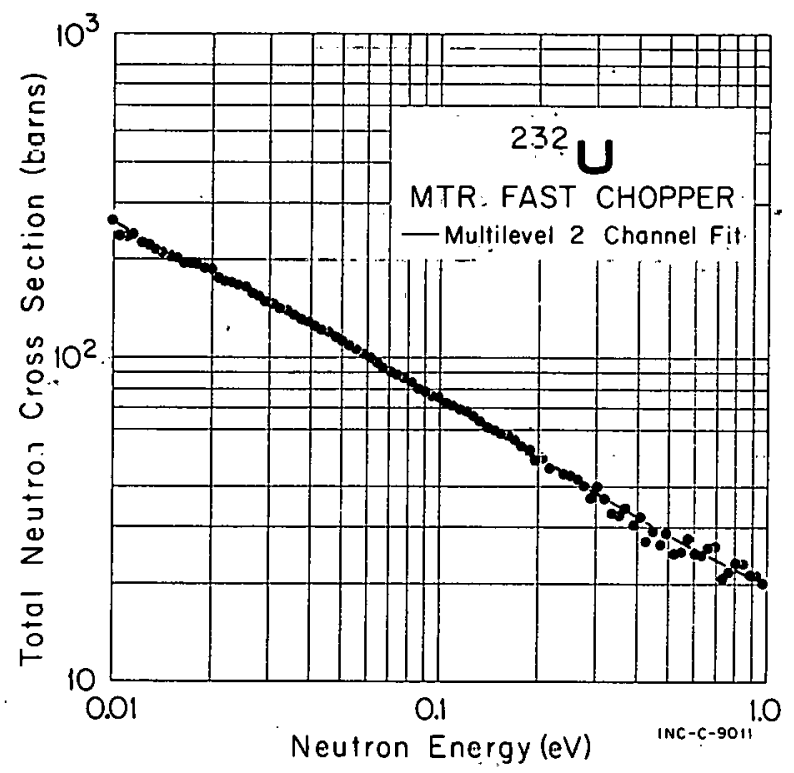

Fig. 1. The total neutron cross section of ${ }^{232} \mathrm{U}$ from 0.01 to $1.0 \mathrm{eV}$. The solid line represents a multilevel two-channel fit to the data. 

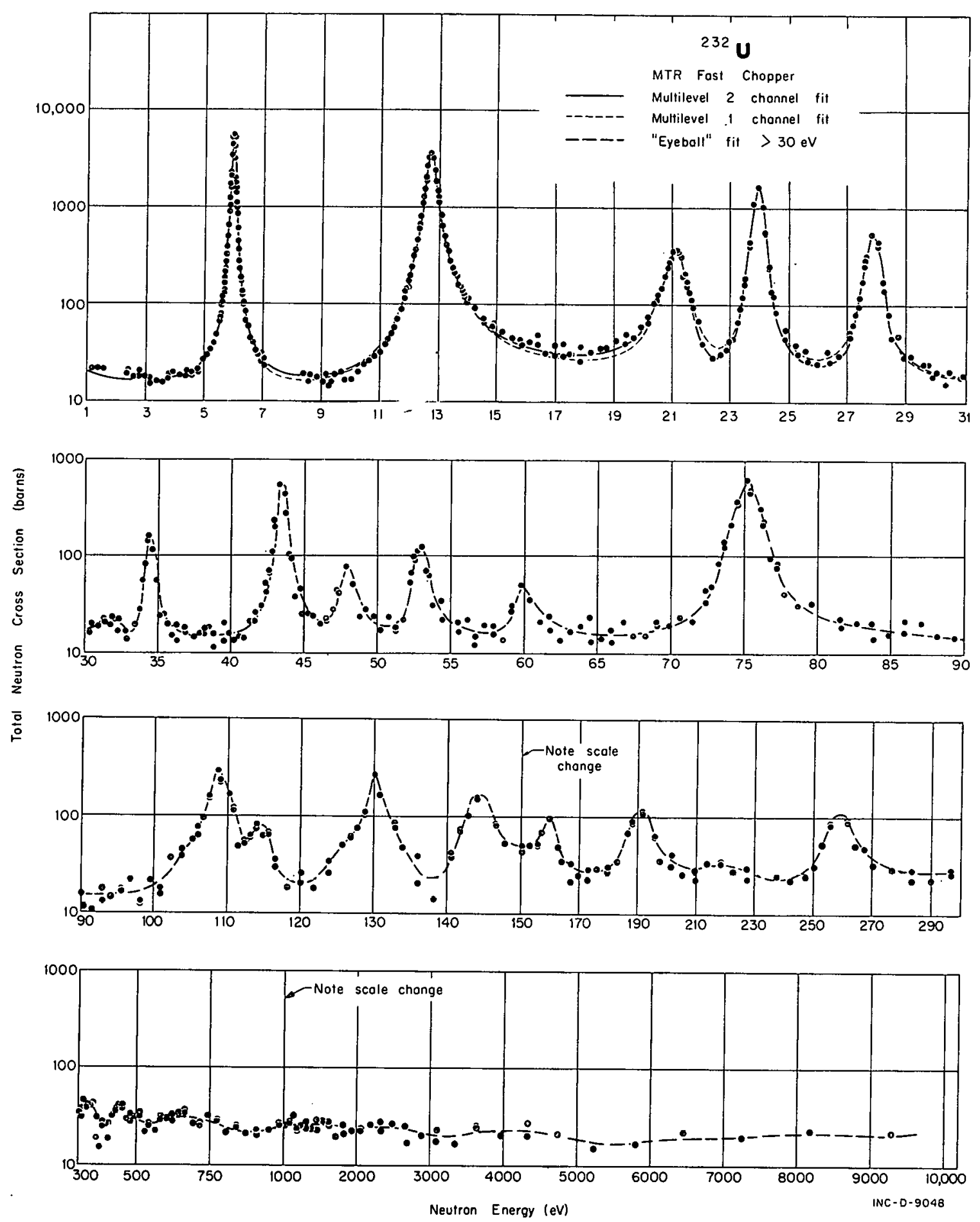

Fig. 2. The total neutron cross section of ${ }^{232}$ U from 1 to $10,000 \mathrm{eV}$. The solid line below $30 \mathrm{cV}$ is a two-chamel multilevel fit to the data: the dashed line below $30 \mathrm{eV}$ is a one-channel multilevel fit. The dashed line above $30 \mathrm{eV}$ is an "eyeball" fit to the data. The data in the regions of 2 and $7.5 \mathrm{eV}$ were omitted because of a ${ }^{22{ }^{T}}$ Th contaminant. 


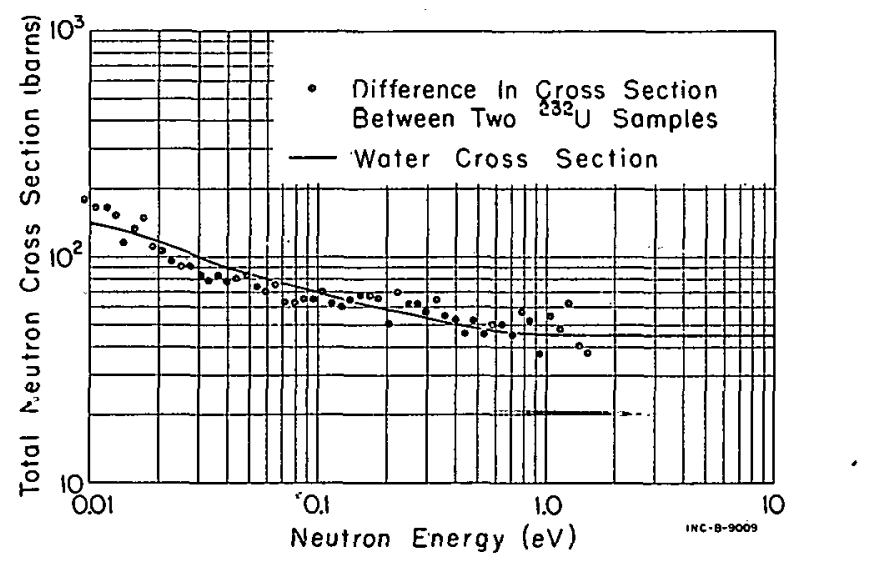

Fig. 3. The difference in cross section between two ${ }^{232} U$ samples having inverse thicknesses of 576.9 and 556.6 barms/atom. The data points have been normalized to the water cross section which is shown by the solid line. 
Appendix A

$$
\text { Tabulation of the }{ }^{232} \mathrm{U} \text { Data }
$$

Operating Condition Using a Flight Path of 20 Meters

\begin{tabular}{|c|c|c|c|c|}
\hline $\begin{array}{l}\text { IBM Run } \\
\text { Number } \\
\end{array}$ & $\begin{array}{l}\text { Rotor } \\
\text { Speed } \\
\text { (rpm) } \\
\end{array}$ & $\begin{array}{l}\text { Channel } \\
\text { Width } \\
\text { ( } \mu \text { sec) } \\
\end{array}$ & $\begin{array}{l}\text { Resolution } \\
\text { ( }{ }^{*} \text { sec/meter) } \\
\end{array}$ & $\begin{array}{c}\text { Sample } \\
\text { Thickness } \\
\text { (Bams/atom) } \\
\end{array}$ \\
\hline 1897 & 3000 & 2 & 0.18 & 287.2 \\
\hline 1954 & 300 & 16 & 2.0 & 287.2 \\
\hline 1958 & 3000 & 2 & 0.18 & $581 \cdot 3$ \\
\hline 1959 & 3000 & 2 & 0.18 & 560.6 \\
\hline 1960 & 6000 & 1 & 0.10 & 287.2 \\
\hline 1961 & 6000 & 1 & 0.10 & $581 \cdot 3$ \\
\hline 1962 & 6000 & 1 & 0.10 & 560.5 \\
\hline 1963 & 6000 & 1 & 0.10 & 2217 \\
\hline \multicolumn{5}{|c|}{$\begin{array}{l}\text { * The following assumptions were made: Triangular neutron burst width } \\
\text { with full-width at half-maximum of } 1.6 \mathrm{\mu sec} \text { at } 6000 \mathrm{mpm} \text {, rectangular } \\
\text { effective counter thickness of } 4.8 \mathrm{~cm} \text { and a Maxwellian distribution of } \\
0.5 \mu \text { sec full-width at half-maximum for the counter jitter. Effect of } \\
\text { detector thickness for measurements above } 1 \mathrm{eV} \text { was computed at } 5 \mathrm{eV} \text {. }\end{array}$} \\
\hline
\end{tabular}




\begin{tabular}{|c|c|c|c|c|c|c|c|}
\hline & IDE & If & CATION & & DATE & DELAY & TRANS, \\
\hline$u=$ & 232 & 000 & 1897 & ODS & $02 / 08 / 65$ & 22.000 & 0.9113 \\
\hline U- & 232 & 000 & 1897 & ODS & $02 / 08 / 65$ & 24.000 & 0.9190 \\
\hline U. & 232 & 000 & 1897 & $O D S$ & $02 / 08 / 65$ & 26.000 & $0.939 ?$ \\
\hline U. & 232 & 000 & 1897 & ODS & $02 / 08 / 65$ & 28.000 & 0.9133 \\
\hline U. & 232 & 000 & 1897 & ODS & $02 / 08 / 65$ & 30.000 & 0.9054 \\
\hline U. & 232 & 000 & 1897 & ODS & $02 / 08 / 65$ & 32.000 & 0.9244 \\
\hline U. & 232 & 000 & 1897 & ODS & $02 / 08 / 65$ & 34.000 & 0.9127 \\
\hline U. & 232 & 000 & 1897 & ODS & $02 / 08 / 65$ & 36.000 & 0.9059 \\
\hline U- & 232 & 000 & 1897 & ODS & $02 / 08 / 65$ & 38.000 & 0.9027 \\
\hline U. & 232 & 000 & 1897 & ODS & $02 / 08 / 65$ & 40.000 & 0.9067 \\
\hline u. & 232 & 000 & 1897 & ODS & $02 / 08 / 65$ & 42.000 & 0.9167 \\
\hline u. & 232 & 000 & 1897 & 005 & $02 / 08 / 65$ & 44.000 & 0.9101 \\
\hline u- & 232 & 000 & 1897 & ODS & $02 / 08 / 65$ & 46.000 & 0.9122 \\
\hline U. & 232 & 000 & 1897 & $O D S$ & $02 / 08 / 65$ & 48.000 & 0.9218 \\
\hline U. & 232 & 000 & 1897 & ODS & $02 / 08 / 65$ & 50.000 & 0.9198 \\
\hline U. & 232 & 000 & 1897 & ODS & $02 / 08 / 65$ & 52.000 & 0.9055 \\
\hline U- & 232 & 000 & 1897 & ons & $02 / 08 / 65$ & 54.000 & 0.9148 \\
\hline u. & 232 & 000 & 1897 & ODS & $02 / 08 / 65$ & 56.000 & 0.8868 \\
\hline U. & 232 & 000 & 1897 & ODS & $02 / 08 / 65$ & 58.000 & 0.9063 \\
\hline$U_{+}$ & 232 & & 1897 & ODS & $02 / 08 / 65$ & 60.000 & 0,8942 \\
\hline U. & 232 & 000 & 1897 & 005 & $02 / 08 / 65$ & 62.000 & 0.9128 \\
\hline u- & 232 & 000 & 1897 & ODS & $02 / 08 / 65$ & 64.000 & 0.8949 \\
\hline Un & 232 & 000 & 1897 & ODS & $02 / 08 / 65$ & 66.000 & 0.8882 \\
\hline u. & 232 & 000 & 1897 & ODS & $02 / 08 / 65$ & 68.000 & 0.8673 \\
\hline u. & 232 & 000 & 1897 & ODS & $02 / 08 / 65$ & 70.000 & 0.8809 \\
\hline U. & 232 & 000 & 1897 & ODS & $02 / 08 / 65$ & 72.000 & 0.9104 \\
\hline u. & 232 & 000 & 1897 & ODS & $02 / 08 / 65$ & 74.000 & 0.9181 \\
\hline U. & 232 & 000 & 1897 & ODS & $02 / 08 / 65$ & 76.000 & 0.8977 \\
\hline U. & 232 & 000 & 1897 & ODS & $02 / 08 / 65$ & 78.000 & 0.8690 \\
\hline U. & 232 & 000 & 1897 & ODS & $02 / 08 / 65$ & 80.000 & 0.8751 \\
\hline u. & 232 & 000 & 1897 & ODS & $02 / 08 / 65$ & 82.000 & 0.8981 \\
\hline u. & 232 & 000 & 1897 & Ons & $02 / 08 / 65$ & 84.000 & 0.9122 \\
\hline U. & 232 & 000 & 1897 & ODS & $02 / 08 / 65$ & 86.000 & 0.9040 \\
\hline u. & 232 & 000 & 1897 & ODS & $02 / 08 / 65$ & 88.000 & 0.8499 \\
\hline u. & 232 & 000 & 1897 & ODS & $02108 / 65$ & 90.000 & 0.8156 \\
\hline$u=$ & 232 & 000 & 1897 & ODS & $02 / 08 / 65$ & 92.000 & 0.8797 \\
\hline u. & 232 & 000 & 1897 & ODS & $02 / 08 / 65$ & 94.000 & 0.9142 \\
\hline U. & 232 & 000 & 1897 & ODS & $02 / 08 / 05$ & 96.000 & 0.9204 \\
\hline u. & 232 & 000 & 1897 & ODS & $02 / 08 / 65$ & 98.000 & 0.8876 \\
\hline U. & 232 & 000 & 1897 & ODS & $02 / 08 / 65$ & 100.000 & 0.9206 \\
\hline U. & 232 & 000 & 18970 & ODS & $02 / 08 / 65$ & 102.000 & 0.8585 \\
\hline u- & 232 & 000 & 18970 & ODS & $02 / 08 / 65$ & 0 & 0.7863 \\
\hline U. & 232 & 000 & 18970 & ODS & $02 / 08 /$ & 106.0 & 0.8511 \\
\hline U. & 232 & 000 & 18970 & ons & $02 / 08 / 65$ & 108.000 & 0.8933 \\
\hline U- & 232 & 000 & 18970 & ons & $02 / 08 / 65$ & 110.000 & 0.8995 \\
\hline U. & 232 & 000 & 1897 & ODS & $02 / 08 / 65$ & 112.0 & 0.8837 \\
\hline U. & 232 & 000 & 18970 & ODS & $02 / 08 / 65$ & 114.000 & 0.8168 \\
\hline U. & 232 & 000 & 1897 & ODS & $02 / 08 / 65$ & 116.000 & 0.8250 \\
\hline U. & 232 & 000 & 18970 & ODS & $02 / 08 / 65$ & 118.000 & 0.8290 \\
\hline u. & 232 & 000 & 1897 & ODS & $02 / 08 / 65$ & 120.000 & 0.7674 \\
\hline u. & 232 & 000 & $1897 \mathrm{C}$ & ODS & $02 / 08 / 65$ & 122.000 & 0.8595 \\
\hline u. & $23 ?$ & 000 & 1897 & ODS & $02 / 08 / 65$ & 124.000 & 0.8688 \\
\hline u. & 232 & 000 & 1897 & ODS & $02 / 08$ & 126. & 0,7388 \\
\hline$U_{n}$ & 232 & 000 & 1897 & ODS & $02 / 08 / 65$ & 128.000 & 0.7890 \\
\hline u. & 232 & 000 & 1897 & ODS & $02 / 08 / 65$ & 130.000 & 0.8797 \\
\hline u. & 232 & 000 & 1897 & ODS & $02 / 08 / 65$ & 132.000 & 0.9113 \\
\hline u. & 232 & 000 & 1897 & ODS & $02 / 0$ & 134. & $0.879_{1}$ \\
\hline u. & 232 & 000 & 1897 & ODS & $02 / 08 / 65$ & 136.000 & 0.7943 \\
\hline ve & 232 & 000 & 1897 & ODS & $02 / 08,65$ & 138,000 & 0.6914 \\
\hline U. & 232 & 000 & 1897 & ODS & $02 / 08 / 65$ & 140.000 & 0,7389 \\
\hline u. & 232 & 000 & 1897 & ODS & $02 / 08 / 65$ & 142.000 & 0,8675 \\
\hline u. & 232 & 000 & 1897 & ODS & $02 / 08 / 65$ & 144.000 & 0,9355 \\
\hline u- & 232 & 000 & 1897 & ODS & $02 / 08 / 65$ & 146.000 & 0.9526 \\
\hline U. & 232 & 000 & 1897 & ODS & 0 & 148.0 & 0.9428 \\
\hline u. & 232 & 000 & 1897 & ODS & 188 & 150.000 & 0.9528 \\
\hline U. & 232 & 000 & 1897 & ODS & $02 / 08 / 65$ & 152.0 & 0.9454 \\
\hline$u=$ & 232 & 000 & 1897 & ODS & $02 / 08 / 65$ & 154.000 & 0,9486 \\
\hline U. & 232 & 000 & 1897 & ODS & $02 / 08 / 65$ & 156.000 & 0.9448 \\
\hline U. & 232 & 000 & 1897 & ODS & $02 / 08 / 65$ & 158.000 & 0.9296 \\
\hline u. & 232 & 000 & 18970 & ODS & $02 / 08 / 65$ & 150.000 & 0.9228 \\
\hline$u=$ & 232 & 000 & 18970 & ODS & $02 / 08 / 65$ & 162.000 & 0.6 \\
\hline$u=$ & 232 & 000 & 1897 & ODS & 0 & 4.000 & \\
\hline u. & 232 & 000 & 1897 & ODS & 0 & 00 & 9 \\
\hline U. & 232 & 000 & 1897 & ODS & 0 & 0 & 838 \\
\hline U. & 232 & 000 & 1897 & ODS & $02 / 08 / 65$ & 170.0 & 0 \\
\hline u. & 232 & 000 & 18970 & ODS & & 172.000 & \\
\hline U. & 232 & 000 & 1897 & ODS & 021 & 174,000 & 0.9285 \\
\hline U. & 232 & 000 & 1897 & ODS & 0 & 176,000 & 0,9489 \\
\hline U. & 232 & 000 & 1897 & ODS & $02 / 08$ & 178.0 & 0.9413 \\
\hline u. & 232 & 000 & 1897 & ODS & $02 / 08 / 65$ & 180.000 & 0.9218 \\
\hline$U=$ & 232 & 000 & 1897 & ODS & $02 / 08 / 65$ & 182.000 & 0.9434 \\
\hline$u=$ & 232 & 000 & 1897 & ODS & $02 / 08 / 65$ & 84.000 & 0.9190 \\
\hline U. & 232 & 000 & 1897 & $O D S$ & 0 & & \\
\hline U. & 232 & 000 & 1897 & ODS & $02 / 08 / 65$ & 188.000 & 0,9 \\
\hline U. & 232 & 000 & 1897 & ODS & $02 / 08 / 65$ & 190.000 & 0.9468 \\
\hline U. & 232 & 000 & 1897 & ODS & $02 / 08 / 65$ & 192.000 & 0.9487 \\
\hline U. & 232 & 000 & 1897 & ODS & 0 & & \\
\hline U. & 232 & 000 & 1897 & ODS & $02 / 08 / 65$ & 196.000 & 0 \\
\hline U. & 232 & 000 & 1897 & ODS & $02 / 08 / 65$ & 198.000 & 0.7796 \\
\hline u. & 232 & 000 & 1897 & ODS & $02 / 08 / 65$ & 200,000 & 0.8306 \\
\hline
\end{tabular}




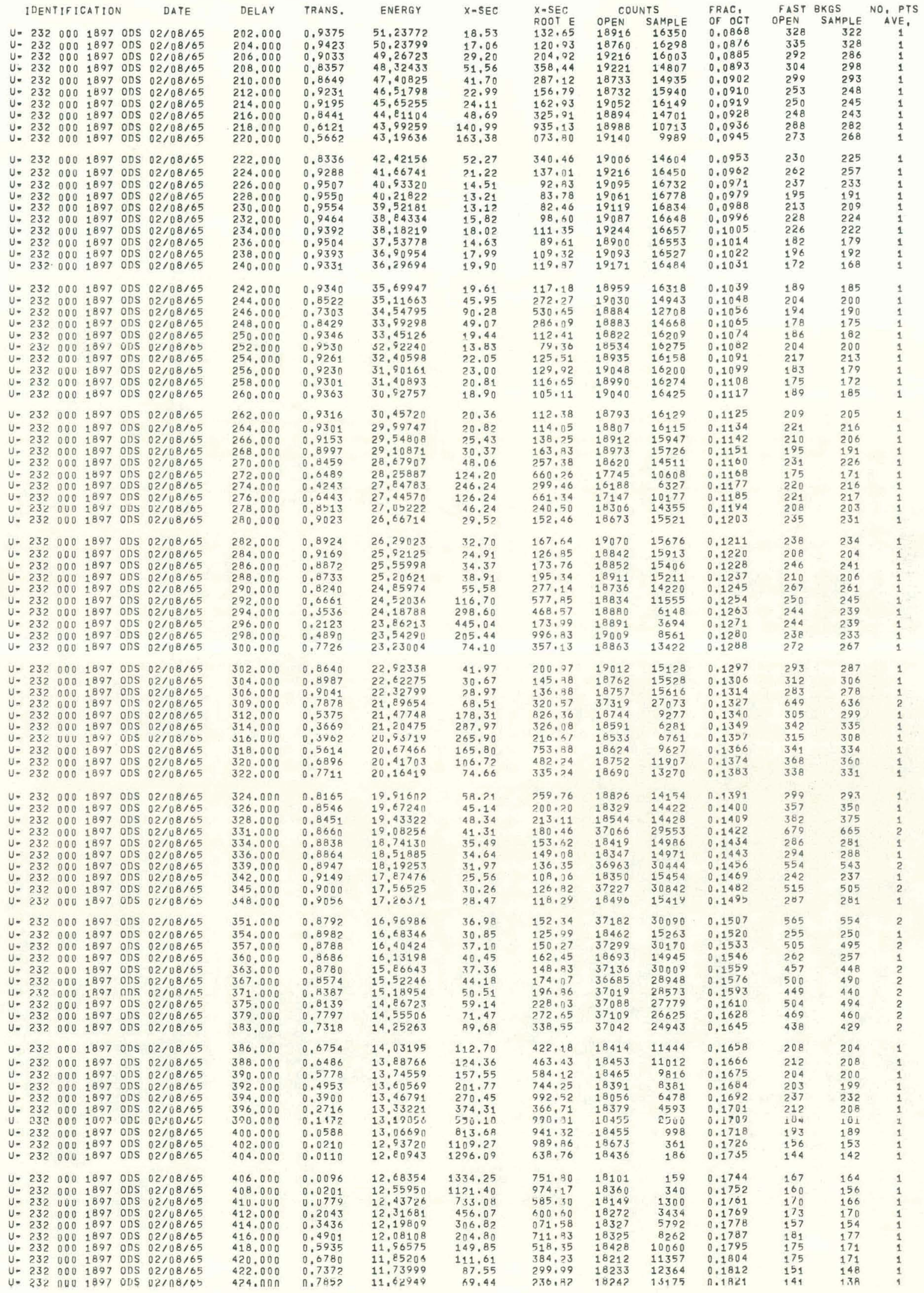




\begin{tabular}{|c|c|c|c|c|c|c|c|c|c|c|c|}
\hline IDENTIFICATION & deLaY & prans. & ENERGy & $x$-sEc & $x$-SEEC & cou & & FRAC, & FAST BY & & NO, PTS \\
\hline$U=2320001897$ ODS $02 / 08 / 65$ & 426,000 & 0.8207 & 11,52055 & 56.75 & $\begin{array}{l}\text { RoOT E } \\
192.63\end{array}$ & $\begin{array}{l}O P E N, \\
18069\end{array}$ & $\begin{array}{l}\text { SAMPLE } \\
13639\end{array}$ & 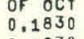 & $\begin{array}{lll}O P E N \\
151\end{array}$ & ${ }_{148}^{A 4 P L}$ & \\
\hline 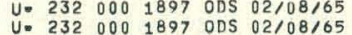 & $\begin{array}{l}428.000 \\
4300000\end{array}$ & $\begin{array}{l}0.8430 \\
0,853\end{array}$ & $\begin{array}{l}11,41314 \\
11,30721\end{array}$ & 43. & & $\begin{array}{l}18109 \\
18147\end{array}$ & 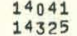 & $\begin{array}{l}0,1838 \\
0,1847\end{array}$ & $\frac{122}{173}$ & $\begin{array}{l}120 \\
170\end{array}$ & \\
\hline 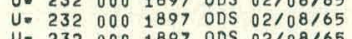 & $\begin{array}{l}430.000 \\
4325000\end{array}$ & $\begin{array}{l}0.6783 \\
0.8768\end{array}$ & 11,20276 & 37.77 & 126.40 & $\begin{array}{l}18124 \\
1824\end{array}$ & 14615 & 0.1855 & 167 & 164 & \\
\hline 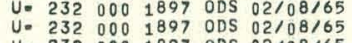 & $\begin{array}{l}435.000 \\
439.000\end{array}$ & $\begin{array}{l}0.8956 \\
0.9050\end{array}$ & $\begin{array}{l}11.04877 \\
10.84835\end{array}$ & $\begin{array}{l}31.68 \\
28.67\end{array}$ & 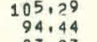 & $\begin{array}{l}36241 \\
36220\end{array}$ & $\begin{array}{l}29850 \\
30144\end{array}$ & $\begin{array}{l}0.1868 \\
0.1885\end{array}-35$ & $\begin{array}{l}295 \\
290\end{array}$ & $\begin{array}{l}289 \\
284\end{array}$ & \\
\hline 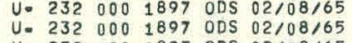 & $\begin{array}{l}443.000 \\
447.000\end{array}$ & $\begin{array}{l}0,9145 \\
0,9231\end{array}$ & $\begin{array}{l}10.65332 \\
10.46359\end{array}$ & $\begin{array}{l}25.68 \\
22.99\end{array}$ & $\begin{array}{l}83,83 \\
74,35\end{array}$ & $\begin{array}{l}36302 \\
35865\end{array}$ & $\begin{array}{l}30528 \\
30444\end{array}$ & $\begin{array}{l}0.1933 \\
0,1920\end{array}-3$ & $\begin{array}{l}247 \\
246 \\
230\end{array}-100$ & 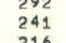 & \\
\hline 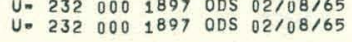 & $\begin{array}{l}451.000 \\
456.000\end{array}$ & $\begin{array}{l}0.9342 \\
0.9445\end{array}$ & $\begin{array}{l}10,27873 \\
10,05456\end{array}$ & $\begin{array}{l}19.56 \\
16.39\end{array}$ & $\begin{array}{l}62.71 \\
51.97\end{array}$ & $\begin{array}{l}36029 \\
53895\end{array}$ & $\begin{array}{l}30949 \\
46517\end{array}$ & 0.1958 & $\begin{array}{l}220 \\
359\end{array}$ & 352 & $\frac{2}{3}$ \\
\hline U. 2320001897 ODS $02 / 08 / 65$ & 461.000 & 0.9444 & 9.83763 & 16.42 & 51.51 & 35941 & 31301 & 0.1980 & 291 & 85 & \\
\hline$u=2320001897$ DDS $02 / 08165$ & $\begin{array}{l}465.000 \\
470.000\end{array}$ & $\begin{array}{l}0.9345 \\
0,9377\end{array}$ & $\begin{array}{ll}9.69611 \\
9.46448\end{array}$ & $\begin{array}{l}19: 8 \\
18:\end{array}$ & $\begin{array}{l}60.51 \\
56,81\end{array}$ & $\begin{array}{l}36022 \\
54124\end{array}$ & $\begin{array}{l}\begin{array}{l}31048 \\
46801\end{array}-1 \\
400\end{array}$ & $\begin{array}{l}0.1997 \\
0.2018\end{array}$ & $\begin{array}{l}2144 \\
344\end{array}$ & $\begin{array}{l}210 \\
337\end{array}$ & \\
\hline 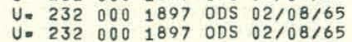 & $\begin{array}{l}475.000 \\
480000\end{array}$ & $\begin{array}{l}0: 9512 \\
0: 9468\end{array}$ & $\begin{array}{l}9,2628 \\
9 ; 07424 \\
9\end{array}$ & $\begin{array}{l}14.36 \\
15.69\end{array}$ & $\begin{array}{l}43,71 \\
47>27\end{array}$ & $\begin{array}{l}35885 \\
53862\end{array}$ & $\begin{array}{l}31475 \\
47064\end{array}$ & $\begin{array}{l}20040 \\
: 2061\end{array}$ & $\begin{array}{l}236, \\
359 \\
396\end{array}$ & 52 & \\
\hline 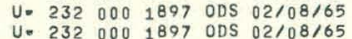 & $\begin{array}{l}486.000 \\
492.000\end{array}$ & $\begin{array}{l}0: 9410 \\
0.9389\end{array}-10$ & $\begin{array}{l}8.855156 \\
8.63699\end{array}$ & $\begin{array}{l}17.47 \\
18.11\end{array}$ & 53.23 & $\begin{array}{l}53637 \\
53658\end{array}$ & 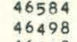 & $\begin{array}{l}.2087 \\
.2113\end{array}$ & 305 & 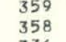 & \\
\hline 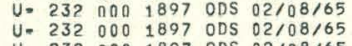 & $\begin{array}{l}498.000 \\
502.000\end{array}$ & $\begin{array}{l}0: 9343 \\
0.9275\end{array}$ & 8.29631 & $\begin{array}{l}19.52 \\
21.61\end{array}$ & 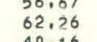 & $\begin{array}{l}3.112 \\
18112\end{array}$ & 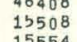 & (2156 & $\begin{array}{l}108 \\
108\end{array}$ & 100 & \\
\hline 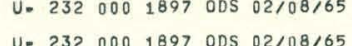 & 506,000 & 0.9418 & 8.16566 & & 21 & 7851 & 5522 & 2173 & 105 & & \\
\hline 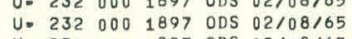 & 500.000 & 0.7410 & & & & & & & & & \\
\hline $89 .>2>>>$ & 510.000 & 941 & 8.03808 & 1.7. & & & & & & & \\
\hline ODS $02 / 0$ & 514.000 & $0,9421>020$ & 7.913 & & & & & & & & \\
\hline 1897 ODS 0210 & 516.000 & $0.92-3$ & 7,852 & & & & & 216 & & & \\
\hline 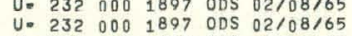 & $\begin{array}{l}518.000 \\
520.000\end{array}$ & $\begin{array}{l}0.9470 \\
0.9322\end{array}$ & 7,73189 & 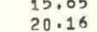 & & 7667 & & 235 & & & \\
\hline 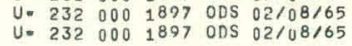 & $\begin{array}{l}522.000 \\
524000\end{array}$ & $\begin{array}{l}0.9059 \\
0.8548\end{array}$ & $\begin{array}{l}7,67276 \\
7,61430\end{array}$ & $\begin{array}{l}28.38 \\
41.72\end{array}$ & 14 & 462 & 34 & 2525 & $\begin{array}{l}111 \\
109\end{array}$ & 109 & \\
\hline U. 2320001897 & 526.000 & 0.8502 & 7.55651 & 46.60 & 8,09 & 17616 & 13819 & 59 & 08 & 106 & \\
\hline CSE 000 107/ UDS $0 E 700703$ & 530.000 & & & & & & & & & & \\
\hline 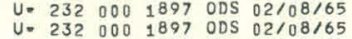 & $\begin{array}{l}532.000 \\
534.000\end{array}$ & 0.9281 & $\begin{array}{l}7,3802 \\
7,33179\end{array}$ & $\begin{array}{l}21.44 \\
27.95\end{array}$ & $\begin{array}{l}58,26 \\
75,69\end{array}$ & $\begin{array}{l}81 \\
34\end{array}$ & 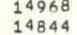 & $\begin{array}{l}0.2285 \\
0.2293\end{array}$ & 107 & $\begin{array}{l}104 \\
105\end{array}-\mathrm{b} \mathrm{b}$ & \\
\hline 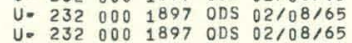 & $\begin{array}{l}536.000 \\
538000\end{array}$ & $\begin{array}{l}0: 9206 \\
0.9185\end{array}$ & $\begin{array}{l}7,2718 \\
7,22317 \\
7,2317\end{array}$ & $\begin{array}{l}23.77 \\
24.43\end{array}$ & $\begin{array}{l}64.13 \\
65,66\end{array}$ & $\begin{array}{l}17576 \\
17515\end{array}$ & $\begin{array}{l}14925 \\
14830\end{array}$ & $\begin{array}{l}0.2302 \\
0,2311\end{array}$ & $\begin{array}{l}108 \\
108\end{array}$ & 06 & \\
\hline 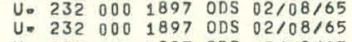 & $\begin{array}{l}540.000 \\
542,000\end{array}$ & $\begin{array}{l}0.9373 \\
0: 9274\end{array}$ & $7: 116977$ & $\begin{array}{l}18.6 \\
21: 6\end{array}$ & $\begin{array}{l}49,81 \\
57778\end{array}$ & 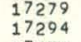 & $\begin{array}{l}14920 \\
14760\end{array}$ & $\begin{array}{l}0.2319 \\
0.2328\end{array}-\mathrm{l}-\mathrm{l}$ & 108 & $\begin{array}{l}106 \\
105\end{array}$ & \\
\hline 1897 & 544.000 & & 7.06472 & 27. & & 52 & & & 106 & 104 & \\
\hline $232 \quad 000 \quad 1897$ oDs $02 / 188 / 65$ & 551.000 & 0. & 6.88 & 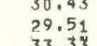 & & 188 & & & & & \\
\hline 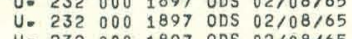 & 559.000 & 0.8671 & 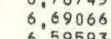 & $40^{\circ}$ & 105.92 & 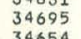 & & & 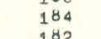 & 年 -20 & \\
\hline $\begin{array}{lll}000 & 1897 & 005 \\
022 / 10\end{array}$ & 567.000 & & 6.50319 & $599^{4}$. & & 60 & & & & & \\
\hline 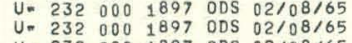 & $\begin{array}{l}571.000 \\
574.000\end{array}$ & $\begin{array}{l}0.7464 \\
0.679\end{array}$ & $\begin{array}{l}6.4240 \\
6,34554\end{array}-1454$ & $\begin{aligned} 84, \\
11 .\end{aligned}$ & 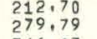 & $\begin{array}{l}346992 \\
17115\end{array}$ & $\begin{array}{l}{ }_{10691}^{23785} \\
1060 \mathrm{l} \mathrm{l}\end{array}$ & $\begin{array}{l}0.2 \\
0.2 \\
0.2\end{array}$ & $\begin{array}{l}78 \\
88\end{array}$ & 86 & \\
\hline 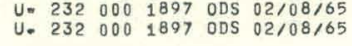 & $\begin{array}{l}576.000 \\
578.000\end{array}$ & $\begin{array}{l}0.6183 \\
0.5456\end{array}$ & $\begin{array}{l}6.30155 \\
6,25802 \\
6.2502\end{array}$ & 174.0 & $\begin{array}{l}346,63 \\
435,32\end{array}$ & $\begin{array}{l}7270 \\
6894\end{array}$ & $\begin{array}{l}9826 \\
8487\end{array}$ & $\begin{array}{l}.2474 \\
2482\end{array}$ & $\begin{array}{l}88 \\
07\end{array}$ & $\begin{array}{l}86 \\
86\end{array}$ & \\
\hline 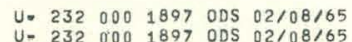 & 580.000 & 4154 & 6.21493 & 52.29 & 8.95 & $\begin{array}{l}17305 \\
71414\end{array}$ & 622 & $1 \frac{1}{9}$ & 86 & $\begin{array}{lll} & \end{array}$ & \\
\hline 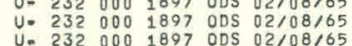 & $\begin{array}{l}582.000 \\
58.000\end{array}$ & 1273 & $\begin{array}{l}0.12509 \\
6,13009\end{array}$ & 592.02 & & & 2025 & 08 & $\begin{array}{r}85 \\
84\end{array}$ & $\begin{array}{l}84 \\
83\end{array}$ & \\
\hline $189700502 / 0$ & 588.000 & 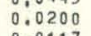 & 6.04697 & 1124.1 & 90 & & & & 83 & & \\
\hline 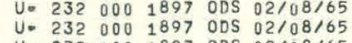 & $\begin{array}{l}590.000 \\
592.000\end{array}$ & $\begin{array}{l}0.0117 \\
0.0084\end{array}$ & $\begin{array}{l}6.00064 \\
5.96553\end{array}$ & $\begin{array}{l}1277.63 \\
1372.55\end{array}-\mathrm{l}$ & $\begin{array}{l}131 ; 11 \\
352,39 \\
352\end{array}$ & $\begin{array}{l}17294 \\
17140\end{array}$ & $\begin{array}{l}186 \\
132\end{array}$ & $0:$ : $-2-1$ & $\begin{array}{l}82 \\
8_{1}\end{array}$ & $\begin{array}{l}80 \\
79\end{array}$ & \\
\hline 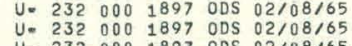 & $\begin{array}{l}594.000 \\
596.000\end{array}$ & $\begin{array}{l}0.0111 \\
0.0331\end{array}$ & $\begin{array}{l}5.9243 \\
5.8573 \\
5.8573\end{array}$ & $\begin{array}{l}1293.74 \\
978.63\end{array} \mathrm{~V}-\mathrm{l}$ & $\begin{array}{l}149,25 \\
374,20 \\
342,23\end{array}$ & 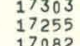 & 520 & & $\begin{array}{l}79 \\
78\end{array}$ & $\begin{array}{l}716 \\
75\end{array}$ & \\
\hline $\begin{array}{llllll}232 & 000 & 1897 & 005 & 02 / 08 / 65 \\
232 & 000 & 1897 & \text { ODS } & 2 / 08 / 65\end{array}$ & 600.000 & 2549 & & 392.52 & & 17000 & 992 & & 76 & 75 & \\
\hline 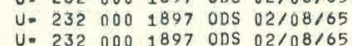 & $\begin{array}{l}602.000 \\
604.000\end{array}$ & 2049 & 5.7689 & $2044-2-3>$ & & 17238 & & & 25 & 74 & \\
\hline $\begin{array}{l}189700502 / 08 / 65 \\
189705\end{array}$ & $\begin{array}{l}804.000 \\
606.000\end{array}$ & 12 & 5.69308 & 年 & 400 & 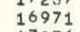 & & & 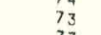 & 10 & \\
\hline 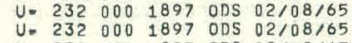 & $\begin{array}{l}608.000 \\
610.000\end{array}$ & $\begin{array}{l}6588 \\
7143\end{array}$ & $\begin{array}{l}5.65569 \\
5,61866\end{array}$ & $\begin{array}{l}1199.85 \\
96.62\end{array}$ & $\begin{array}{l}285,02 \\
229,03\end{array}$ & $\begin{array}{l}177036 \\
17014\end{array}$ & $\begin{array}{l}10346 \\
11205\end{array}$ & & 72 & 71 & \\
\hline$\left\{\begin{array}{l}0210 \\
02 / 0\end{array} \mid\right.$ & $\begin{array}{l}612.000 \\
615\end{array}$ & 7653 & 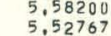 & $\begin{array}{l}76 \\
65 \\
65\end{array}$ & & & & & 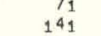 & 38 & \\
\hline 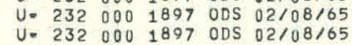 & $\begin{array}{l}620.000 \\
626.000\end{array}$ & $\begin{array}{l}850 \\
872 \\
872-1030\end{array}$ & $\begin{array}{l}5.43888 \\
5.33512\end{array}$ & $\begin{array}{l}46.57 \\
39.12\end{array}$ & $\begin{aligned} 108.61 \\
90.36\end{aligned}$ & 1526 & 39 & & $\begin{array}{l}207 \\
203\end{array}$ & $\begin{array}{l}203 \\
199\end{array}$ & \\
\hline 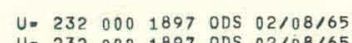 & 632.000 & 8901 & 5. & 33 & & $s$ & & & 199 & 19 & \\
\hline $\begin{array}{l}50210 \\
0210 \\
0\end{array}$ & $\begin{array}{l}638.000 \\
648.000\end{array}$ & 0 & 4: & 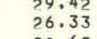 & & $\begin{array}{c}30 \\
117\end{array}$ & & & & & \\
\hline $\begin{array}{l}232 \\
232 \\
232000\end{array}$ & $\begin{array}{l}662.000 \\
676.000\end{array}$ & $\begin{array}{l}0.0 .307 \\
0.9334\end{array}$ & $\begin{array}{l}4.77664 \\
4.57508\end{array}-1708$ & $\begin{array}{l}20.5 \\
19\end{array}$ & & $\begin{array}{l}119448 \\
1.15177\end{array}$ & 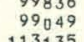 & & 年 & 41 & \\
\hline 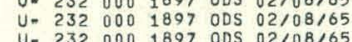 & 707.000 & & $\begin{array}{l}4.18267 \\
3.9855\end{array}$ & $\begin{array}{l}18 \\
18\end{array}-3$ & & 15 & $1+10 e^{2}$ & & $\begin{array}{l}44 \\
48\end{array}$ & $\begin{array}{l}438 \\
474\end{array}$ & \\
\hline 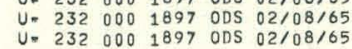 & $\begin{array}{l}743.000 \\
764.000\end{array}$ & 99432 & $\begin{array}{l}3,78717 \\
3,58184\end{array}$ & $\begin{array}{l}16.79 \\
15.59\end{array}$ & $\begin{array}{l}32.67 \\
29.50\end{array}$ & $\begin{array}{l}158570 \\
172201\end{array}$ & $\begin{array}{l}137 \\
149 \\
149\end{array}$ & $\begin{array}{l}0.3191 \\
0.3281\end{array}-31$ & $\begin{array}{l}546 \\
543\end{array}$ & $\begin{array}{l}506 \\
532\end{array}$ & 1. \\
\hline $25 n 2 / n 8 / 65$ & 787.000 & $946 n$ & 3,37 & 15. & & 0 & & 30 & 506 & 555 & \\
\hline $502 / 08 / 65$ & $\begin{array}{l}811,000 \\
837,000\end{array}$ & & $\begin{array}{l}33,17 \\
2,98\end{array}$ & & & & & & $\begin{array}{l}5 \\
6 \\
6\end{array}$ & $\begin{array}{c}b 3 \\
59 \\
59\end{array}-100$ & \\
\hline $\begin{array}{lll}23 & 0 \\
23 & 0 & 0\end{array}$ & $\begin{array}{l}867,000 \\
900.000\end{array}$ & $0_{0}^{0}-(-2-3-1$ & $\begin{array}{l}2,781144 \\
2,5112\end{array}$ & & & $\begin{array}{l}2338 \\
2428\end{array}$ & 2 & & $\begin{array}{l}600 \\
673\end{array}$ & $\begin{array}{l}646 \\
659\end{array}$ & \\
\hline 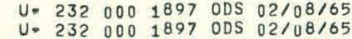 & $\begin{array}{l}937.000 \\
980.000\end{array}$ & $\begin{array}{l}9.9361 \\
9362\end{array}$ & $\begin{array}{l}2,38130 \\
2,17691\end{array}$ & $\begin{array}{l}18.97 \\
18.95\end{array}$ & $\begin{array}{l}29.27 \\
27.96\end{array}$ & $\begin{array}{l}27180 \\
306838\end{array}-180$ & & & $\begin{array}{l}765 \\
862\end{array}$ & $\begin{array}{l}750 \\
845\end{array}$ & \\
\hline 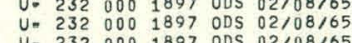 & $\begin{array}{l}\begin{array}{l}1029.000 \\
1085,000\end{array} \\
1000\end{array}$ & & $\begin{array}{l}11,4752 \\
1,7596 \\
1,5750\end{array}$ & & & & & & $\begin{array}{l}9167 \\
1670 \\
1520\end{array}$ & $\begin{array}{l}1141 \\
1144\end{array}$ & \\
\hline & & & & & & & & & & & \\
\hline 2320001897 ODS $02 / 08 / 105$ & $\begin{array}{l}1233.000 \\
1334.000\end{array}$ & & $\begin{array}{l}1,0720 \\
1.17485 \\
0.97280\end{array}$ & $\begin{array}{l}21.31 \\
21.28 \\
23.14\end{array}$ & & & & & $\begin{array}{l}2300 \\
2303 \\
3393\end{array}$ & ${ }_{2313}^{2313}$ & \\
\hline & & & & & & & & & & & \\
\hline
\end{tabular}


RUN 1954

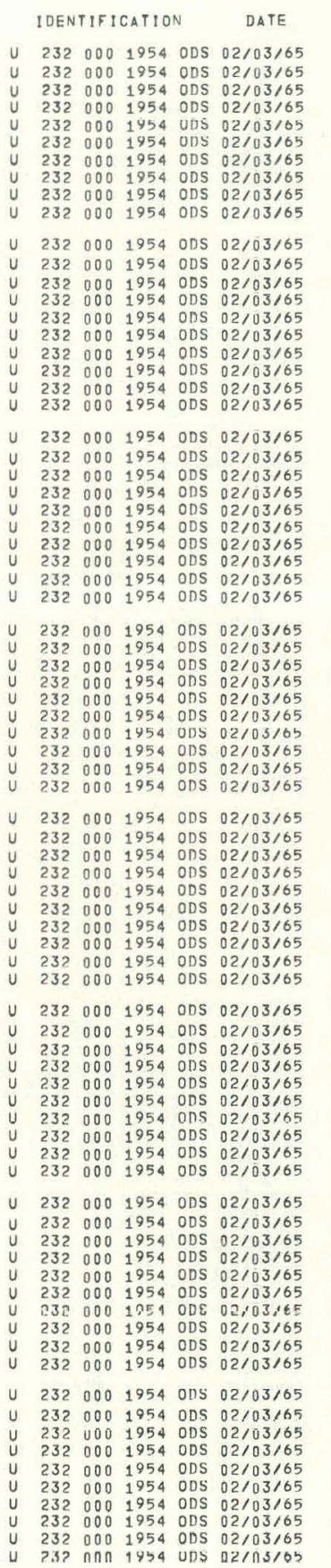

\begin{tabular}{|c|c|c|c|}
\hline & IANS, & ENERGY & \\
\hline .000 & 0,8264 & 18.92986 & 6.75 \\
\hline & $\begin{array}{l}0.8609 \\
0.8074\end{array}$ & $\begin{array}{l}17,2229020 \\
15,74783\end{array}$ & .45 \\
\hline & $\begin{array}{l}0.003754 \\
0.3544\end{array}$ & 13.30556 & $\begin{array}{l}119.19 \\
281.40\end{array}$ \\
\hline & $\begin{array}{l}0.7345 \\
0.7345 \\
0.824\end{array}$ & 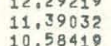 & \\
\hline & $\begin{array}{l}0.9278 \\
0.9455\end{array}$ & 9,86070 & $\begin{array}{l}21.55 \\
1670 \\
16.70\end{array}$ \\
\hline & 0.9467 & 8,61972 & \\
\hline & 0.905 & $\begin{array}{l}8.08530 \\
7.59908\end{array}$ & \\
\hline & 0.8972 & 7,15543 & \\
\hline & $\begin{array}{l}0.81567 \\
0.5727\end{array}$ & $\begin{array}{l}8.377722 \\
60,37428\end{array}$ & \\
\hline 0000 & $\begin{array}{l}0.3228 \\
0.5257\end{array}$ & $\begin{array}{l}\begin{array}{l}6.03488 \\
5 \\
5\end{array} 71939 \\
0\end{array}$ & \\
\hline $\begin{array}{l}.000 \\
000\end{array}$ & $\begin{array}{l}0.7791 \\
0.8861\end{array}$ & $\begin{array}{l}5,42800 \\
5,15833\end{array}$ & $\frac{69}{72}$ \\
\hline .000 & $\begin{array}{l}0.9117 \\
0.9277\end{array}$ & $\begin{array}{l}4,90827 \\
4,67596\end{array}$ & \\
\hline & $\begin{array}{l}0.9342 \\
0.93592\end{array}$ & $\begin{array}{l}4.45976 \\
4.2584\end{array}$ & \\
\hline & $\begin{array}{l}0.9350 \\
0.9506\end{array}$ & $\begin{array}{r}4.0701 \\
4.0703 \\
7.804\end{array}$ & \\
\hline & $\begin{array}{l}0.9564 \\
0.9356 \\
0.934\end{array}$ & $\begin{array}{l}0.72924 \\
3 \\
3\end{array}$ & \\
\hline .000 & $\begin{array}{l}0.9534 \\
0.94727\end{array}$ & $\begin{array}{l}3.29304 \\
3.116455\end{array}$ & 96 \\
\hline & 0.9375 & 3,04343 & \\
\hline 300 & 3420 & $\begin{array}{r}2,27438 \\
2,71904\end{array}$ & \\
\hline 300 & $\begin{array}{l}0.9599 \\
0.9543\end{array}$ & 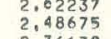 & \\
\hline 000 & $\begin{array}{l}0.9324 \\
0.9549\end{array}$ & $\begin{array}{l}2,201397 \\
2,24527\end{array}$ & \\
\hline & $\begin{array}{l}0.8932 \\
0.7834\end{array}$ & $\begin{array}{l}2.03736 \\
2.94403\end{array}$ & \\
\hline & 0,7356 & 1.85700 & \\
\hline & 35 & $\begin{array}{l}1,775 \\
1,699\end{array}$ & \\
\hline & $\begin{array}{l}0.9335 \\
0.9271\end{array}$ & $\begin{array}{l}1.60551 \\
1.51904\end{array}$ & \\
\hline & $\begin{array}{l}0.9425 \\
0.9736\end{array}$ & $\begin{array}{r}1,45888 \\
1,40186\end{array}$ & \\
\hline .000 & $\begin{array}{l}0.9317 \\
0.9286\end{array}$ & $\begin{array}{l}1.33111 \\
1.26595\end{array}$ & \\
\hline .000 & 0.9336 & 1,20479 & 19.74 \\
\hline .000 & 0.9476 & $\begin{array}{l}1,14827 \\
1.09563\end{array}$ & \\
\hline & $\because:$ & $\begin{array}{l}1.03478 \\
1.097885\end{array}$ & \\
\hline & $\begin{array}{l}0.928 \\
0.927\end{array}$ & $\begin{array}{l}0.93732 \\
0.09837\end{array}$ & \\
\hline & $\begin{array}{l}0.9222 \\
0.921\end{array}$ & $\begin{array}{l}n .85300 \\
0.1090\end{array}$ & \\
\hline & $\begin{array}{l}0.9264 \\
0.9296\end{array}$ & $\begin{array}{l}0.7200 \\
0.73577\end{array}$ & \\
\hline & 0.911 & 0.70202 & \\
\hline & 0.8 & $\begin{array}{l}0.635 \\
0.635\end{array}$ & \\
\hline & & 0.57 & \\
\hline & & 0.52 & \\
\hline & $\begin{array}{l}0.9116 \\
0.9028\end{array}$ & $\begin{array}{l}0.47676 \\
0.45223\end{array}$ & \\
\hline & & 0,4 & \\
\hline & & $\begin{array}{l}0,4 \\
0,3\end{array}$ & \\
\hline & & $\begin{array}{l}0.37085 \\
0.35394\end{array}$ & \\
\hline & & $\begin{array}{l}0.33816 \\
0.32138\end{array}$ & \\
\hline & & & \\
\hline & & 0 & \\
\hline
\end{tabular}

\begin{tabular}{|c|c|c|c|c|c|c|}
\hline $\mathbb{E}_{i \in}$ & & & FRAC: & $\begin{array}{c}\text { FAST } \\
\text { OAPEN }\end{array}$ & KGS & \\
\hline & $\begin{array}{l}\text { OPEN } \\
19079\end{array}$ & $\begin{array}{l}\text { AMPLLE } \\
14517\end{array}$ & 0.0164 & $\begin{array}{l}\text { PPEN, } \\
257\end{array}$ & $\begin{array}{l}\text { SAMPLE } \\
257\end{array}$ & \\
\hline & $\begin{array}{l}19159 \\
1959\end{array}$ & $\begin{array}{r}15254 \\
1554\end{array}$ & 0.0172 & 260 & $\begin{array}{r}260 \\
220\end{array}$ & \\
\hline & $\begin{array}{l}19252 \\
1952\end{array}$ & 11698 & 0.0188 & 209 & 209 & \\
\hline $\begin{array}{l}6.243 \\
6,57\end{array}$ & 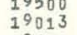 & $\begin{array}{l}6734 \\
7 y s 0\end{array}$ & $\begin{array}{l}0.0196 \\
0.0204\end{array}$ & $\begin{array}{l}100 \\
17_{1}\end{array}$ & $\begin{array}{l}180 \\
171_{1}\end{array}$ & \\
\hline $\begin{array}{l}0.92 \\
6.94\end{array}$ & $\begin{array}{l}19430 \\
19317 \\
1919\end{array}$ & $\begin{array}{l}13126 \\
15675 \\
1575\end{array}$ & $\begin{array}{l}0.0122 \\
0.0220\end{array}$ & $\begin{array}{l}137 \\
148\end{array}$ & $\begin{array}{l}157 \\
148\end{array}$ & \\
\hline 50.68 & $\begin{array}{l}19198 \\
19247\end{array}$ & $\begin{array}{l}10425 \\
16750\end{array}$ & $\begin{array}{l}0.028 \\
0.0235\end{array}$ & $\begin{array}{l}141 \\
138\end{array}$ & $\begin{array}{l}141 \\
138\end{array}$ & \\
\hline 6.1 & ${ }_{19191}$ & 16769 & 0.0243 & 118 & 118 & \\
\hline & 1905 & & & 118 & & \\
\hline 83.37 & $\begin{array}{l}19100 \\
19347\end{array}$ & $\begin{array}{l}10013 \\
15986\end{array}$ & $\begin{array}{l}0.0267 \\
0.026\end{array}$ & $\begin{array}{l}107 \\
115\end{array}$ & $\begin{array}{l}107 \\
115\end{array}$ & \\
\hline $\begin{array}{l}151,200 \\
404,32\end{array}$ & $\begin{array}{l}19288 \\
19061\end{array}$ & $\begin{array}{l}14298 \\
10032\end{array}$ & $\begin{array}{l}0.0275 \\
0.0283\end{array}$ & $\begin{array}{l}108 \\
94 \\
94\end{array}$ & $\begin{array}{c}108 \\
94 \\
94\end{array}$ & \\
\hline $\begin{array}{l}798.11 \\
441.71\end{array}$ & $\begin{array}{l}19254 \\
19366\end{array}$ & $\begin{array}{r}3721 \\
9382\end{array}$ & $\begin{array}{l}0.02911 \\
0.0299\end{array}$ & $\begin{array}{l}88 \\
88\end{array}$ & $\begin{array}{l}88 \\
88\end{array}$ & \\
\hline $\begin{array}{r}166.988 \\
78,85\end{array}$ & $\begin{array}{l}19041 \\
19089\end{array}$ & $\begin{array}{l}13682 \\
15592\end{array}$ & $\begin{array}{l}0.0307 \\
0.0315\end{array}$ & $\begin{array}{l}\frac{111}{114} \\
114\end{array}$ & $\begin{array}{l}\frac{111}{114} \\
114\end{array}$ & \\
\hline 58.79 & 19211 & 16139 & 0.0323 & 129 & 129 & \\
\hline & 1887 & $\begin{array}{l}16246 \\
16252\end{array}$ & 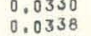 & 12 & $\begin{array}{l}129 \\
144\end{array}$ & \\
\hline .61 & $\begin{array}{l}18619 \\
18519\end{array}$ & $\begin{array}{l}15898 \\
16189\end{array}$ & 0.0346 & 131 & 131 & \\
\hline & $\begin{array}{l}18801 \\
18901\end{array}$ & $\begin{array}{l}16583 \\
1683\end{array}$ & $\begin{array}{l}0.0362 \\
0.037\end{array}$ & 123 & 123 & \\
\hline $\begin{array}{l}36,91 \\
36,20\end{array}$ & 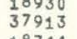 & $\begin{array}{l}162090 \\
32581\end{array}$ & $\begin{array}{l}0.03870 \\
0.0382\end{array}$ & $\begin{array}{l}\frac{139}{308} \\
308\end{array}$ & $\begin{array}{l}139 \\
308 \\
3\end{array}$ & \\
\hline $\begin{array}{l}\begin{array}{l}24.85 \\
30.17\end{array} \\
3.17\end{array}$ & $\begin{array}{l}18744 \\
18756\end{array}$ & $\begin{array}{l}16464 \\
16304\end{array}$ & $\begin{array}{l}0.0394 \\
0.0402\end{array}$ & $\begin{array}{l}\frac{157}{161} \\
161\end{array}$ & $\begin{array}{l}157 \\
161\end{array}$ & \\
\hline $\begin{array}{l}32.33 \\
27.24\end{array}$ & $\begin{array}{l}19000 \\
37355\end{array}$ & $\begin{array}{l}16429 \\
32584\end{array}$ & $\begin{array}{l}0.0410 \\
0.0281\end{array}$ & $\begin{array}{l}141 \\
281 \\
282\end{array}$ & $\begin{array}{l}1_{281} \\
282\end{array}$ & \\
\hline $\begin{array}{l}2^{88,31} \\
2_{33442}^{4}\end{array}$ & $\begin{array}{l}18764 \\
18594\end{array}$ & $\begin{array}{l}16304 \\
1608\end{array}$ & $\begin{array}{l}0.0040 \\
0.043\end{array}$ & $\begin{array}{l}122 \\
1{ }_{1}^{2} 2\end{array}$ & $\begin{array}{l}122 \\
113\end{array}$ & \\
\hline $\begin{array}{l}1.19 \\
10.19\end{array}$ & $\begin{array}{r}37349 \\
18606\end{array}$ & $\begin{array}{l}32825 \\
15859\end{array}$ & $\begin{array}{l}0.0453 \\
0.065\end{array}$ & $\begin{array}{l}259 \\
126\end{array}$ & & \\
\hline 34.03 & $\begin{array}{r}36885 \\
3657\end{array}$ & 31883 & 0.0477 & 202 & 202 & \\
\hline $\begin{array}{r}46,31 \\
126.00\end{array}$ & $\begin{array}{l}369934 \\
6934\end{array}$ & 30198 & 0.0501 & 149 & 149 & \\
\hline 120,19 & 867 & 24573 & 0524 & 146 & & \\
\hline & 18326 & 233 & & 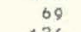 & & \\
\hline $\begin{aligned} 26.56 \\
25.06\end{aligned}$ & $\begin{array}{l}\begin{array}{r}365550 \\
36673\end{array} \\
36\end{array}$ & $\begin{array}{r}308844 \\
31206\end{array}$ & $\begin{array}{l}0.0558 \\
0.0564\end{array}$ & $\begin{array}{l}126 \\
132\end{array}$ & $\begin{array}{l}126 \\
132\end{array}$ & \\
\hline & 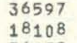 & $\begin{array}{l}311015 \\
15621\end{array}$ & $\begin{array}{l}0.0580 \\
0.0592\end{array}$ & $\begin{array}{r}133 \\
44\end{array}$ & 133 & \\
\hline $\begin{array}{l}21.91 \\
23 \\
236 \\
4.66\end{array}$ & $\begin{array}{l}36479 \\
36642\end{array}$ & $\begin{array}{l}31342 \\
311129\end{array}$ & $\begin{array}{l}0.0603 \\
0.0019\end{array}$ & 115 & 115 & \\
\hline $\begin{array}{l}23,92 \\
21.06\end{array}$ & 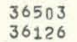 & $\begin{array}{l}31120 \\
30975\end{array}$ & $\begin{array}{l}0.0653 \\
0.0651\end{array}$ & $\begin{array}{c}104 \\
98\end{array}$ & $\begin{array}{r}104 \\
98\end{array}$ & \\
\hline 16,57 & 35865 & 31221 & 0.0667 & 94 & 94 & \\
\hline & & $\begin{array}{l}31046 \\
05685\end{array}$ & & 40 & & \\
\hline 20.05 & $\begin{array}{l}33591 \\
355948\end{array}$ & 30792 & $\left\{\begin{array}{l}0 \\
0,7222\end{array}\right.$ & $\begin{array}{l}101 \\
102\end{array}$ & $\begin{array}{l}101 \\
102\end{array}$ & \\
\hline & 35522 & $\begin{array}{r}30292 \\
30294\end{array}$ & ${ }_{4}^{\circ}$ & 10 & ${ }_{122}$ & \\
\hline $\begin{array}{c}.53 \\
25 \\
25\end{array}$ & $\begin{array}{l}53732 \\
35670\end{array}$ & $\begin{array}{r}45559 \\
30199\end{array}$ & $\begin{array}{l}0.0774 \\
0.0793\end{array}$ & $\begin{array}{l}142 \\
108\end{array}$ & $\begin{array}{l}142 \\
108\end{array}$ & \\
\hline $\begin{array}{l}19,99 \\
17,99\end{array}$ & $\begin{array}{l}531755 \\
35367\end{array}$ & $\begin{array}{l}452511 \\
30209\end{array}$ & $\begin{array}{l}0.0813 \\
0,0833\end{array}$ & $\begin{array}{l}\begin{array}{l}170 \\
104\end{array}\end{array}$ & $\begin{array}{l}\begin{array}{l}170 \\
104\end{array}\end{array}$ & \\
\hline 22.18 & 53070 & 44462 & 0.0853 & 146 & 146 & \\
\hline & $\begin{array}{l}52 \\
35 \\
35\end{array}$ & 29624 & 0896 & $9 t$ & 90 & \\
\hline & & & & 135 & & \\
\hline $\begin{array}{l}21,12 \\
18,84\end{array}$ & $\begin{array}{l}52611 \\
52587\end{array}$ & $\begin{array}{r}438866 \\
44164\end{array}$ & $\begin{array}{l}0.0940 \\
0.0964\end{array}$ & $\begin{array}{l}\begin{array}{l}115 \\
133\end{array} \\
133\end{array}$ & $\begin{array}{l}\begin{array}{l}115 \\
133\end{array} \\
133\end{array}$ & \\
\hline $\begin{array}{l}18.17 \\
20.61\end{array}$ & $\begin{array}{l}520078 \\
52980\end{array}$ & $\begin{array}{l}4.37766 \\
43887\end{array}$ & $\begin{array}{l}n+1887 \\
0 ; 1011\end{array}$ & 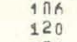 & 0 & \\
\hline $\begin{array}{l}18,35 \\
19 ; 74\end{array}$ & $\begin{array}{l}52883 \\
70400\end{array}$ & $\begin{array}{l}\begin{array}{r}441888 \\
58242\end{array} \\
58\end{array}$ & $\begin{array}{l}0.1035 \\
0.1063\end{array}$ & $\begin{array}{l}\frac{124}{149} \\
149\end{array}$ & & \\
\hline 17.77 & 52572 & 43813 & 1090 & 02 & 102 & \\
\hline & & & & & & \\
\hline & & & & & & \\
\hline & 71233 & 8054 & $\begin{array}{l}172 \\
12 \\
\end{array}$ & $\begin{array}{l}150 \\
153\end{array}$ & $\begin{array}{l}150 \\
153\end{array}$ & \\
\hline & & & & & & \\
\hline & & & & & & \\
\hline
\end{tabular}




\begin{tabular}{|c|c|c|c|c|c|c|c|c|c|c|c|c|c|c|c|c|}
\hline \multicolumn{5}{|c|}{ IDENTIFICATION } & \multirow{2}{*}{$\begin{array}{c}\text { DATE } \\
02 / 03 / 65\end{array}$} & \multirow{2}{*}{$\begin{array}{c}\text { DELAY } \\
2804,000\end{array}$} & TRANS. & \multirow[t]{2}{*}{ ENERGY } & \multirow{2}{*}{$\begin{array}{l}X-S E C \\
42.59\end{array}$} & $\begin{array}{l}X \cdot S E C \\
\text { ROOT E }\end{array}$ & OPEN & $\begin{array}{l}\text { NTS } \\
\text { SAMPLE }\end{array}$ & $\begin{array}{l}\text { FRAC, } \\
\text { OF OCT }\end{array}$ & $\begin{array}{l}\text { FAST } \\
\text { OPEN }\end{array}$ & $\begin{array}{l}\text { BKGS } \\
\text { SAMPLE }\end{array}$ & $\begin{array}{c}\text { No, PTS } \\
\text { AVE, }\end{array}$ \\
\hline U & 232 & 000 & 1954 & ODS & & & 0.8622 & & & 21.94 & 80514 & 63340 & 0.1387 & 190 & 190 & 8 \\
\hline u & 232 & 000 & 1954 & ODS & $02 / 03 / 65$ & 2876.000 & 0.8591 & 0.25226 & 43.63 & 21.91 & 106622 & 83540 & 0.1423 & 211 & 211 & 10 \\
\hline u & 232 & 000 & 1954 & ODS & $02 / 03 / 65$ & 2948.000 & & 0,24000 & 43.69 & 21,41 & 90595 & 70936 & 0,1458 & 202 & 202 & 8 \\
\hline u & 232 & 000 & 1954 & ODS & $02 / 03 / 65$ & 3100.000 & 0.8525 & 0.21712 & 45.85 & 21.36 & 135429 & 105155 & 0.1533 & 212 & 212 & 10 \\
\hline u & 232 & 000 & 1954 & ODS & $02 / 03 / 65$ & 3180.000 & 0.8424 & 0.20633 & 49.27 & 22.38 & 151737 & 116369 & 0.1573 & 222 & 222 & 10 \\
\hline u & 232 & 000 & 1954 & ODS & $02 / 03 / 65$ & 3260.000 & 0.8442 & 0.19633 & 48.65 & 21.55 & 168099 & $129_{141}$ & 0.1613 & 211 & 211 & 10 \\
\hline u & 232 & 000 & 1954 & ODS & $02 / 03 / 65$ & 3340.000 & 0.8343 & 0,18704 & 52.01 & 22.50 & 186853 & 141818 & 0.1652 & 174 & 174 & 10 \\
\hline U & 232 & 000 & 1954 & ODS & $02 / 03 / 65$ & 3420.000 & 0.8300 & 0.17839 & 53.51 & 22.60 & 209509 & 158123 & 0,1692 & 186 & 186 & 10 \\
\hline$u$ & 232 & 000 & 1954 & ODS & $02 / 03 / 65$ & 3500.000 & 0.8223 & 0.17033 & 56.18 & 23.19 & 234379 & 175203 & 0.1731 & 177 & 177 & 10 \\
\hline$u$ & 232 & 000 & 1954 & ODS & $02 / 03 / 65$ & 3588,000 & 0,8177 & 0,16208 & 57.81 & 23,27 & 316928 & 235499 & 0.1775 & 185 & 185 & 12 \\
\hline$u$ & 232 & 000 & 1954 & ODS & $02 / 03 / 65$ & 3676.000 & 0.8154 & 0.15441 & 58.62 & 23,03 & 293843 & 217647 & 0.1818 & 161 & 161 & 10 \\
\hline$u$ & 232 & 000 & 1954 & ODS & $02 / 03 / 65$ & 3764.000 & 0.8120 & 0.14727 & 59.80 & 22.95 & 387553 & 285755 & 0.1862 & 218 & 218 & 12 \\
\hline u & 232 & 000 & 1954 & ODS & $02 / 03 / 65$ & 3860.000 & 0.8091 & 0.14004 & 60.82 & 22.76 & 425714 & 312610 & 0,1909 & 204 & 204 & 12 \\
\hline u & 232 & 000 & 1954 & ODS & $02 / 03 / 65$ & 3956.000 & 0.8002 & 0,13332 & 64.03 & 23.38 & 469158 & 340495 & 0,1957 & 171 & 171 & 12 \\
\hline$u$ & 232 & 000 & 1954 & ODS & $02 / 03 / 65$ & 4052.000 & 0.7929 & 0.12708 & 66.64 & 23,76 & 509870 & 366480 & 0.2004 & 164 & 164 & 12 \\
\hline u & 232 & 000 & 1954 & ODS & $02 / 03 / 65$ & 4148.000 & 0.7881 & 0,12127 & 68.40 & 23,82 & 546151 & 389944 & 0.2052 & 178 & 178 & 12 \\
\hline u & 232 & 000 & 1954 & ODS & $02 / 03 / 65$ & 4252.000 & 0.7840 & 0,11541 & 69.88 & 23,74 & 080380 & 483006 & 0,2103 & 208 & 208 & 14 \\
\hline$u$ & 232 & 000 & 1954 & ODS & $02 / 03 / 65$ & 4356.000 & 0,7787 & 0.10996 & 71.82 & 23.82 & 616717 & 434604 & 0.2155 & 172 & 172 & 12 \\
\hline$u$ & 232 & 000 & 1954 & ODS & $02 / 03 / 65$ & 4460.000 & 0,7764 & 0,10489 & 72.68 & 23.54 & 752772 & 528584 & 0.2206 & 203 & 203 & 14 \\
\hline$u$ & 232 & 000 & 1954 & ODS & $02 / 03 / 65$ & 4572,000 & 0.7660 & 0.09982 & 76.55 & 24.19 & 789500 & 546604 & 0.2262 & 201 & 201 & 14 \\
\hline$u$ & 232 & 000 & 1954 & ODS & $02 / 03 / 65$ & 4684.000 & 0.7658 & 0,09510 & 76.62 & 23.63 & 815193 & 563851 & 0,2317 & 190 & 190 & 14 \\
\hline u & 232 & 000 & 1954 & & $02 / 03 / 65$ & 4804.000 & 0.7580 & 0,09041 & 79.59 & 23,93 & 964899 & 660031 & 0.2376 & 216 & 216 & 16 \\
\hline u & 232 & 000 & 1954 & ODS & $02 / 03 / 65$ & 4924.000 & 0.7554 & 0.08606 & 80.56 & 23.63 & 858393 & 584751 & 0.2436 & 195 & 195 & 14 \\
\hline u & 232 & 000 & 1954 & ODS & $02 / 03 / 65$ & 5044.000 & 0.7464 & 0.08201 & 84.02 & 24.06 & 1000662 & 673001 & 0.2495 & 206 & 206 & 16 \\
\hline$u$ & 232 & 000 & 1954 & ODS & $02 / 03 / 65$ & 5172.000 & 0.7393 & 0.07800 & 86.73 & 24,22 & 1001241 & 666457 & 0,2558 & 185 & 185 & 16 \\
\hline$u$ & 232 & 000 & 1954 & ODS & $02 / 03 / 65$ & 5300.000 & 0.7326 & 0.07428 & 89.36 & 24,36 & 996876 & 656866 & 0.2622 & 183 & 183 & 16 \\
\hline u & 232 & 000 & 1954 & ODS & $02 / 03 / 65$ & 5428.000 & 0.7277 & 0,07082 & 91.29 & 24.29 & 990768 & 647855 & 0.2685 & 178 & 178 & 16 \\
\hline$u$ & 232 & 000 & 1954 & ODS & $02 / 03 / 65$ & 5584.000 & 0.7213 & 0.06740 & 93.83 & 24,36 & 1093156 & 707804 & 0.2752 & 212 & 212 & 18 \\
\hline U & 232 & 000 & 1954 & ODS & $02 / 03 / 65$ & 5700.000 & 0.7146 & 0.06422 & $96.5 n$ & 24.45 & 955820 & 612543 & 0,2820 & 190 & 190 & 16 \\
\hline$u$ & 232 & 000 & 1954 & ODS & $02 / 03 / 65$ & 5836,000 & 0,7071 & 0,06126 & 99.54 & 24,64 & 1053983 & 667680 & 0.2887 & 179 & 179 & 18 \\
\hline$u$ & 232 & 000 & 1954 & ODS & $02 / 03 / 65$ & 5980.000 & 0.7000 & 0.05835 & 102.43 & 24,74 & 1004711 & 629445 & 0,2958 & 209 & 209 & 18 \\
\hline u & 232 & 000 & 1954 & ODS & $02 / 03 / 65$ & 6132.000 & 0.6933 & 0.05549 & 105.20 & 24,78 & 1063479 & 659163 & 0,3033 & 217 & 217 & 20 \\
\hline U & 232 & 000 & 1954 & ODS & $02 / 03 / 65$ & 6284.000 & 0.6830 & 0.05284 & 109.48 & 25,17 & 917742 & 559811 & 0.3108 & 160 & 160 & 18 \\
\hline u & 232 & 000 & 1954 & ODS & $02 / 03 / 65$ & 6436.000 & 0.6769 & 0.05037 & 112.09 & 25,16 & 959102 & 579141 & 0.3184 & 231 & 231 & 20 \\
\hline u & 232 & 000 & 1954 & ODS & $02 / 03 / 65$ & 6596.000 & 0.66 & 0.04796 & 115.29 & 25.25 & 895515 & 534043 & 0.3263 & 216 & 216 & 20 \\
\hline u & 232 & 000 & 1954 & ODS & $02 / 03 / 65$ & 6764.000 & 0.6592 & 0,04561 & 119.70 & 25.56 & 906739 & 531748 & 0.3346 & 221 & 221 & 22 \\
\hline u & 232 & 000 & 1954 & ODS & $02 / 03 / 65$ & 6932,000 & 0,6538 & 0,04342 & 122.04 & 25,43 & 795814 & 462254 & 0,3429 & 219 & 219 & 20 \\
\hline u & 232 & 000 & 1954 & ODS & $02 / 03 / 65$ & 7100,000 & 0.6468 & 0,04139 & 125,12 & 25,46 & 818032 & 469427 & 0.3512 & 212 & 212 & 22 \\
\hline U & 232 & 000 & 1954 & ODS & $02 / 03 / 65$ & 7276.000 & 0.6399 & 0.03941 & 128.20 & 25.45 & 743793 & 421669 & 0.3599 & 210 & 210 & 22 \\
\hline$u$ & 232 & 000 & 1954 & ODS & $02 / 03 / 65$ & 7452.000 & 0.6328 & 0,03757 & 131.44 & 25,48 & 681187 & 381290 & 0,3686 & 245 & 245 & 22 \\
\hline u & 232 & 000 & 1954 & ODS & $02 / 03 / 65$ & 7636.000 & 0.6236 & 0.03578 & 135.64 & 25.66 & 664775 & 366156 & 0.3777 & 254 & 254 & 24 \\
\hline u & 232 & 000 & 1954 & ODS & $02 / 03 / 65$ & 7828.000 & 0.0162 & 0.03405 & 139.04 & 25.66 & 627794 & 341186 & 0.3872 & 222 & 222 & 24 \\
\hline u & 232 & 000 & 1954 & ODS & $02 / 03 / 65$ & 8020.000 & 0.0119 & 0.03244 & 141.07 & 25,41 & 572029 & 308221 & 0.3967 & 253 & 253 & 24 \\
\hline u & 232 & 000 & 1954 & ODS & $02 / 03 / 65$ & 8220,000 & 0,5996 & 0,03088 & 146.89 & 25,81 & 550309 & 290108 & 0,4066 & 242 & 242 & 26 \\
\hline u & 232 & 000 & 1954 & ODS & $02 / 03 / 65$ & 8428.000 & 0.5952 & 0,02937 & 148.99 & 25.54 & 510660 & 266730 & 0.4169 & 273 & 273 & 26 \\
\hline u & 232 & 000 & 1954 & $O D S$ & $02 / 03 / 65$ & & 0.5822 & 0,02798 & 155.37 & 25,99 & 446078 & 227370 & 0.4272 & 233 & 233 & 26 \\
\hline u & 232 & 000 & 1954 & ODS & $02 / 03 / 65$ & 8844.000 & 0.5772 & 0.02668 & 157.85 & 25.78 & 381442 & 192319 & 0,4375 & 264 & 264 & 26 \\
\hline u & 232 & 000 & 1954 & ODS & $02 / 03 / 65$ & 9060.000 & 0.5647 & 0.02542 & 164.11 & 26.17 & 356263 & 175335 & 0.4482 & 259 & 259 & 28 \\
\hline u & 232 & 000 & 1954 & ODS & $02 / 03 / 65$ & 9284,000 & 0,5584 & 0.02421 & 167,32 & 26.03 & 334582 & 162457 & 0.4592 & 263 & 263 & 28 \\
\hline$u$ & 232 & 000 & 1954 & ODS & $02 / 03 / 65$ & 9516,000 & 0.5504 & 0,02304 & 171.51 & 26.03 & 317707 & 151666 & 0.4707 & 285 & 285 & 30 \\
\hline u & 232 & 000 & 1954 & ODS & $02 / 03 / 65$ & 9756.000 & 5488 & 0.02 & 1 & 5.51 & 267264 & 126 & 0.4 & 285 & 285 & 30 \\
\hline u & 232 & 000 & 1954 & ODS & $02 / 03 / 65$ & 9996.000 & 0.5433 & 0.02 & 175.24 & 25.32 & 221773 & 103983 & 0.4945 & 308 & 308 & 30 \\
\hline u & 232 & 000 & 1954 & ODS & $02 / 03 / 65$ & 10236,000 & 0,5201 & 0,01991 & 187.77 & 26,50 & 191962 & 85927 & 0.5063 & 293 & 293 & 30 \\
\hline u & 232 & 000 & 1954 & ODS & $02 / 03 / 65$ & 10484.000 & 0.5972 & 0,01898 & 189,33 & 26,09 & 181238 & 80399 & 0,5186 & 268 & 268 & 32 \\
\hline u & 232 & 000 & 1954 & ODS & $02 / 03 / 65$ & 10748.000 & 0.5099 & 0.01806 & 193.46 & 26.00 & 160428 & 69875 & 0.5317 & 300 & 300 & 34 \\
\hline u & 232 & 000 & 1954 & ODS & $02 / 03 / 65$ & 11020.000 & 0.5045 & 0.01718 & 196.52 & 25.76 & 136373 & 58524 & 0.5451 & 339 & 339 & 34 \\
\hline u & 232 & 000 & 1954 & ODS & $02 / 03 / 65$ & 11292.000 & 0.5060 & 0.01636 & 195.63 & 25.03 & 112751 & 48337 & 0.5586 & 341 & 341 & 34 \\
\hline u & 232 & 000 & 1954 & ODS & 02103165 & 11564.000 & 0.4929 & 0.01560 & 203.19 & 25.38 & 93909 & 39049 & 0.5720 & 307 & 307 & 34 \\
\hline$u$ & 232 & 000 & 1954 & ODS & $02 / 03 / 65$ & 11844,000 & 0.4901 & 0,01487 & 204.79 & 24,98 & 88201 & 36306 & 0.5859 & 322 & 322 & 36 \\
\hline U & 232 & 000 & 1954 & ODS & $02 / 03 / 65$ & 12140.000 & 0.4784 & 0.01416 & 211.75 & 25.19 & 79792 & 31891 & 0,6005 & 370 & 370 & 38 \\
\hline u & 232 & 000 & 1954 & $O D S$ & $02 / 03 / 65$ & 12444,000 & 0.471 & 13 & 216.12 & 25.09 & 72165 & 28245 & 0.6156 & 340 & 340 & 38 \\
\hline u & 232 & 000 & 1954 & ODS & $02 / 03 / 65$ & 12748.000 & & 0,012 & 222.64 & 25.23 & 59934 & 22801 & 0.6306 & 350 & 350 & 38 \\
\hline u & 232 & 000 & 1954 & ODS & $02 / 03 / 65$ & 13060.000 & 0.4538 & 0.01223 & 226.92 & 25.10 & 50015 & 18633 & 0,6460 & 399 & 399 & 40 \\
\hline u & 232 & 000 & 1954 & ODS & $02 / 03 / 65$ & 13388.000 & 0.4337 & 0.01164 & 239.94 & 25.89 & 39133 & 13843 & 0.6623 & 434 & 434 & $4 ?$ \\
\hline u & 232 & 000 & 1954 & ODS & $02 / 03 / 65$ & 13724.000 & 0.4374 & 0.01108 & 237.5 & 25.00 & 26747 & 9478 & 0.678 & 432 & $43 ?$ & 42 \\
\hline 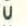 & 232 & 000 & 1954 & ODS & $02 / 03 / 65$ & 14060.000 & 0.4381 & 0.01055 & 237.06 & 24,35 & 17310 & 6101 & 0.6955 & 440 & 440 & 42 \\
\hline u & 232 & 000 & 1954 & ODS & $02 / 03 / 65$ & 14404.000 & 0.3961 & 0.01008 & 265.97 & 26.67 & 11717 & 3935 & 0.7125 & 475 & 475 & 44 \\
\hline
\end{tabular}


RUN 1958

\begin{tabular}{|c|c|c|c|c|c|c|c|c|c|c|c|c|c|c|c|c|}
\hline & IDEN & TIFIC & Tis & & DATE & DELAY & TRANS, & ENERGY & $x-$ SEC & $\begin{array}{l}X \text { X-SEC } \\
\text { ROOT E }\end{array}$ & OPEN & $\begin{array}{l}\text { NTS } \\
\text { SAMPLE }\end{array}$ & $\begin{array}{l}\text { FRAC, } \\
\text { OF OCT }\end{array}$ & $\begin{array}{l}\text { FAST } \\
\text { OPEN }\end{array}$ & $\begin{array}{l}\text { BKGS } \\
\text { SAMPLE }\end{array}$ & $\begin{array}{c}\text { NO, PTS } \\
\text { AVE. }\end{array}$ \\
\hline u= & 232 & 000 & 1958 & ODS & $02 / 08 / 65$ & 26.000 & 0.9587 & 3092,75739 & 24,54 & 1364,75 & 72474 & 64435 & 0,0098 & 21948 & 21729 & 2 \\
\hline U- & 232 & 000 & 1958 & ODS & $02 / 08 / 65$ & 28,000 & 0.9442 & 2666.71426 & 33.36 & 1722.62 & 68155 & 59682 & 0,0105 & 19475 & 19280 & 2 \\
\hline u. & 232 & 000 & 1958 & ODS & $02 / 08 / 65$ & 30.000 & 0,9559 & 2323.00443 & 26.22 & 1263,80 & 68196 & 60453 & 0,0113 & 16346 & 16182 & 2 \\
\hline U. & 232 & 000 & 1958 & ODS & $02 / 08 / 65$ & 32.000 & 0.9457 & 2041.70313 & 32.43 & 1465,40 & 72375 & 63475 & 0,0120 & 12994 & 12864 & 2 \\
\hline U. & 232 & 000 & 1958 & ODS & $02 / 08 / 65$ & 34.000 & 0,9594 & 1808.56746 & 24.10 & 1024,86 & 73878 & 65727 & 0.0128 & 12263 & 12140 & 2 \\
\hline U. & 232 & 000 & 1958 & ODS & $02 / 08 / 65$ & 36.000 & 0.9539 & 1613,19753 & 27.44 & 1102.07 & 74050 & 85501 & 0.0135 & 10977 & 10867 & 2 \\
\hline U. & 232 & 000 & 1958 & ODS & $02 / 08 / 65$ & 38,000 & 0,9474 & 1447,85594 & 31.43 & 1195,95 & 73987 & 64997 & 0,0143 & 9461 & 9366 & 2 \\
\hline u= & 232 & 000 & 1958 & ODS & $02 / 08 / 65$ & 40.000 & 0,9550 & 1306.68999 & 26.79 & 968,44 & 72082 & 63829 & 0.0150 & 8362 & 8278 & 2 \\
\hline U. & 232 & 000 & 1958 & ODS & $02 / 08 / 65$ & 42,000 & 0,9509 & 1185,20634 & 29.25 & 1006,84 & 70169 & 61872 & 0.0158 & 7788 & 7710 & $=$ \\
\hline U: & 232 & 000 & 1958 & ODS & $02 / 08 / 65$ & 44,000 & 0,9523 & 1079,90909 & 28.40 & 933,26 & 69886 & 61711 & 0.0165 & 6685 & 6618 & 2 \\
\hline U. & 232 & 000 & 1958 & ODS & $02 / 08 / 65$ & 46,000 & 0,9498 & 988,04536 & 29.94 & 941,13 & 71559 & 63020 & 0,0173 & 5721 & 5664 & 2 \\
\hline$y=$ & $\begin{array}{l}232 \\
232\end{array}$ & 000 & $\begin{array}{l}1958 \\
1958\end{array}$ & ODS & $\begin{array}{l}02 / 08 / 65 \\
02 / 08 / 65\end{array}$ & 48,000 & $\begin{array}{l}0.9614 \\
0.9646\end{array}$ & $\begin{array}{l}907,42361 \\
836,28159\end{array}$ & 22.91 & $\begin{array}{l}690.13 \\
606.54\end{array}$ & 70382 & 62736 & 0.0180 & 5603 & 5547 & ? \\
\hline u. & 232 & 000 & $\begin{array}{l}1958 \\
1958\end{array}$ & ODS & $02 / 08 / 65$ & $\begin{array}{l}50.000 \\
52.000\end{array}$ & $\begin{array}{l}0.9646 \\
0.9618\end{array}$ & $\begin{array}{l}836,28159 \\
773,18935\end{array}$ & $\begin{array}{l}20.97 \\
22.61\end{array}$ & $\begin{array}{l}606.54 \\
628.80\end{array}$ & $\begin{array}{l}70115 \\
69329\end{array}$ & $\begin{array}{l}62705 \\
61827\end{array}$ & 0.0168 & 5737 & 5680 & 2 \\
\hline u. & 232 & 000 & 1958 & ODS & $02 / 08 / 65$ & 54,000 & 0,9417 & 716,97668 & 34.89 & 934,27 & 69916 & $\begin{array}{l}61827 \\
61046\end{array}$ & $\begin{array}{l}0.0195 \\
0,0203\end{array}$ & $\begin{array}{l}5079 \\
4820\end{array}$ & $\begin{array}{l}5028 \\
4772\end{array}$ & $\begin{array}{l}2 \\
2\end{array}$ \\
\hline u. & 232 & 000 & 1958 & ODS & $02 / 08 / 65$ & 56.000 & 0.9359 & 666.67857 & 38,49 & 993.90 & 70537 & 61207 & 0.0210 & 3993 & 3954 & 2 \\
\hline$U=$ & 232 & 000 & 1958 & ODS & $02 / 08 / 65$ & 58.000 & 0.9408 & 621.49345 & 35.48 & 884.63 & 70.282 & 61301 & 0.0218 & 4200 & 4158 & 2 \\
\hline$u=$ & 232 & 000 & 1958 & ODS & $02 / 08 / 65$ & 60.000 & 0.9453 & 580.75111 & 32.69 & 787.75 & 69827 & 61197 & 0.0225 & 3779 & 3741 & 2 \\
\hline$U=$ & , 232 & 000 & 1958 & ODS & $02 / 08 / 05$ & 62,000 & 0,9530 & 543.88761 & 27.94 & 652.13 & 68240 & 00296 & 0.0233 & 3956 & 3917 & \\
\hline U= & 232 & 000 & 1958 & ODS & $02 / 08 / 65$ & 64.000 & 0.9338 & 510.42578 & 39.81 & 899.44 & 67943 & 58819 & 0.0240 & 3843 & 3804 & 2 \\
\hline$v=$ & 232 & 000 & 1958 & ODS & $02 / 08 / 65$ & 66,000 & 0.9530 & 479.95959 & 28,01 & 613.69 & 66962 & 59156 & 0.0248 & 3381 & 3347 & 2 \\
\hline u. & 232 & 000 & 1958 & ODS & $02 / 08 / 65$ & 68.000 & 0.9310 & 452.14186 & 41.55 & 883.50 & 65782 & 56775 & 0.0255 & 3329 & 3296 & 2 \\
\hline U. & 232 & 000 & 1958 & ODS & $02 / 08 / 65$ & 70.000 & 0.9400 & 426.67428 & 35.98 & 743.11 & 62829 & 54748 & 0.0263 & 2940 & 2910 & 2 \\
\hline$U=$ & 232 & 000 & 1958 & ODS & $02 / 08 / 65$ & 72.000 & 0,9465 & 403,29938 & 31.94 & 641.49 & 56866 & 49897 & 0.0270 & 3063 & 3032 & 2 \\
\hline$u=$ & 232 & 000 & 1958 & ODS & $02 / 08 / 65$ & 74.000 & 0.9581 & 381.79401 & 24.88 & 486.06 & 53242 & 47287 & 0.0278 & 2814 & 2786 & 2 \\
\hline U. & 232 & 000 & 1958 & ODS & $02 / 08 / 65$ & 76.000 & 0.9487 & 361,96399 & 30,60 & 582.18 & 43483 & 38241 & 0.0286 & 2580 & 2554 & 2 \\
\hline U. & 232 & 000 & 1958 & ODS & $02 / 08 / 65$ & 78.000 & 0.9449 & 343.63971 & 32.97 & 611.10 & 34829 & 30506 & 0.0293 & 2504 & 2479 & 2 \\
\hline U. & 232 & 000 & 1958 & ODS & $02 / 08 / 65$ & 80.000 & 0,9374 & 326.67250 & 37.56 & 678.83 & 43070 & 37425 & 0.0301 & 2456 & 2431 & 2 \\
\hline U. & 232 & 000 & 1958 & ODS & $02 / 08 / 65$ & 82,000 & 0,9335 & 310.93159 & 40.00 & 705.32 & 54997 & 47589 & 0,0308 & 2780 & 2752 & 2 \\
\hline U. & 232 & 000 & 1958 & ODS & $02 / 08 / 65$ & 84,000 & 0.9460 & 296.30159 & 32.29 & 555.91 & 60096 & 52694 & 0,0316 & 3030 & 2999 & 2 \\
\hline u. & $\begin{array}{l}232 \\
232\end{array}$ & $\begin{array}{l}000 \\
000\end{array}$ & 1958 & ODS & $02 / 08 / 65$ & 86.000 & 0.9609 & 282,68037 & 23.21 & 390.31 & 62997 & 6 & 0. & 3084 & 3053 & 2 \\
\hline & $\begin{array}{l}232 \\
232\end{array}$ & 000 & 1958 & ODS & 021 & 0 & 0.9 & 269 & 44.56 & 732.10 & 64932 & 55744 & 0.0331 & 3593 & 3557 & 2 \\
\hline$u=$ & $\begin{array}{l}232 \\
232\end{array}$ & $\begin{array}{l}000 \\
000\end{array}$ & 1958 & ODS & $02 / 08 / 05$ & 90.000 & 0.8968 & 258.11160 & 63.32 & 1017.28 & 67218 & 55873 & 0.0338 & 3698 & 3661 & 2 \\
\hline U. & $\begin{array}{l}232 \\
232\end{array}$ & $\begin{array}{l}000 \\
000\end{array}$ & 1958 & ODS & $02 / 08 / 65$ & 92,000 & 0.9244 & 247.01134 & 45.68 & 717.87 & 66537 & 57011 & 0.0346 & 4007 & 3967 & 2 \\
\hline U. & 232 & $\begin{array}{l}000 \\
000\end{array}$ & 1958 & ODS & $02 / 08 / 65$ & 94.000 & 0.9533 & 236.61204 & 27.80 & 427,59 & 64949 & 57387 & 0.0353 & 4331 & 4288 & 2 \\
\hline U. & 232 & $\begin{array}{l}000 \\
000\end{array}$ & $\begin{array}{l}1958 \\
1958\end{array}$ & $\begin{array}{l}\text { ODS } \\
\text { ODS }\end{array}$ & $\begin{array}{l}02 / 08 / 65 \\
02 / 08 / 65\end{array}$ & 96,000 & 0,9544 & $\begin{array}{l}226.85590 \\
217.69096\end{array}$ & 27.13 & 408.62 & 65164 & 57643 & 0.0361 & 4454 & 4409 & 2 \\
\hline U. & 232 & 000 & 1958 & ODS & $02 / 08 / 65$ & $\begin{array}{r}98,000 \\
100,000\end{array}$ & $\begin{array}{l}0,9382 \\
0,9392\end{array}$ & $\begin{array}{l}217.69096 \\
209.07040\end{array}$ & 37.08 & 547.08 & 66693 & 57993 & 0.0368 & 4315 & 4272 & 2 \\
\hline u- & 232 & 000 & 1958 & ODS & $02 / 08 / 65$ & 02.000 & 0.9283 & 200,95194 & $\begin{array}{l}36,46 \\
43,26\end{array}$ & $\begin{array}{l}527,20 \\
613,23\end{array}$ & $\begin{array}{l}66593 \\
66597\end{array}$ & $\begin{array}{l}57967 \\
57296\end{array}$ & 0.0376 & 4842 & 4793 & 2 \\
\hline U- & 232 & 000 & 1958 & ODS & $02 / 08 / 65$ & 104.000 & 0,8935 & 193.29734 & $\begin{array}{l}43,26 \\
65,48\end{array}$ & $\begin{array}{l}613,23 \\
910,38\end{array}$ & $\begin{array}{l}66597 \\
65741\end{array}$ & $\begin{array}{l}57296 \\
54437\end{array}$ & $\begin{array}{l}0.0383 \\
0.0391\end{array}$ & $\begin{array}{l}4495 \\
4564\end{array}$ & $\begin{array}{l}4450 \\
4518\end{array}$ & 2 \\
\hline$u=$ & 232 & 000 & 1958 & ODS & $02 / 08 / 65$ & 106.000 & 0.88 & 1 & & & & & 0,0081 & 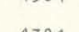 & 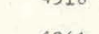 & \\
\hline u. & 232 & 000 & 1958 & ODS & $02 / 08 / 65$ & 108,000 & 0.9360 & 4417 & $\begin{array}{l}71.27 \\
38,47\end{array}$ & $\begin{array}{l}972,22 \\
515,08\end{array}$ & $\begin{array}{l}67664 \\
67329\end{array}$ & & 0.0398 & $\begin{array}{l}4304 \\
4537\end{array}$ & $\begin{array}{l}4261 \\
4499\end{array}$ & 2 \\
\hline v. & 232 & 000 & 1958 & ODS & $02 / 08 / 65$ & 110.000 & 0,9526 & 172.78545 & 28,23 & 371,13 & 66252 & $\begin{array}{l}58402 \\
58488\end{array}$ & $\begin{array}{l}0,0406 \\
0,0413\end{array}$ & $\begin{array}{l}4537 \\
4221\end{array}$ & $\begin{array}{l}4491 \\
4178\end{array}$ & 2 \\
\hline$u=$ & 232 & 000 & 1958 & ODS & $02 / 08 / 65$ & 112,000 & 0.9448 & 166.66964 & 33.01 & 426,12 & 65044 & 56951 & 0.0421 & 4337 & 4293 & 2 \\
\hline U. & 232 & 000 & 1958 & ODS & $02 / 08 / 65$ & 114,000 & 0,9048 & 160.87288 & 58,16 & 737.64 & 664 & 30 & 0.0 & 7 & 3957 & 2 \\
\hline$U=$ & 232 & 000 & 1958 & ODS & $02 / 08 / 65$ & 16.000 & 0.8916 & 7336 & 66.70 & 831,42 & 66167 & 54670 & 0.0436 & 4057 & 4017 & 2 \\
\hline$u=$ & 232 & 000 & 1958 & ODS & $02 / 08 / 65$ & 118,000 & 0.9000 & 150.15111 & 61,23 & 750.28 & 65498 & 54628 & 0.0443 & 3953 & 3913 & 2 \\
\hline$U=$ & 232 & 000 & 1958 & ODS & $02 / 08 / 65$ & 120.000 & 0.8519 & 145 & 93,14 & 1122.32 & 63960 & 50495 & 0.0 & 3646 & 3610 & 2 \\
\hline U. & 232 & 000 & 1958 & ODS & $02 / 08 / 65$ & 122.000 & 0.9054 & 140.46654 & 57.8 & 685.03 & 62770 & 52661 & 0.0 & 3557 & 3521 & 2 \\
\hline ve & 232 & 000 & 1958 & ODS & $02 / 08 / 65$ & 124.000 & 0.9184 & 135.97190 & 49.47 & 576.90 & 62915 & 53544 & 0.0460 & 3393 & 3359 & 2 \\
\hline U. & 232 & 000 & 1958 & ODS & $02 / 08 / 65$ & 126.000 & 0.8427 & 131.68959 & 99.50 & 1141.81 & 62153 & 48533 & 0.0473 & $\$ 163$ & 3131 & 2 \\
\hline U. & 232 & 000 & 1958 & ODS & $02 / 08 / 65$ & 128,000 & 0.8 & 12 & 82. & 47 & $\begin{array}{l}62 \\
59\end{array}$ & 49 & 0. & 2801 & 2773 & 2 \\
\hline$U=$ & 232 & 000 & 1958 & ODS & $02 / 0$ & & & & & & 59 & & & 2677 & 2650 & 2 \\
\hline$U=$ & 232 & 000 & 1958 & ODS & $02 / 08 / 65$ & 32.000 & 0.9576 & 119 , & 25. & 27 & 59832 & 530 & & & 2355 & 2 \\
\hline U- & 232 & 000 & 1958 & ODS & $02 / 08 / 65$ & 134.000 & 0.9131 & & 52. & 570. & 65017 & 55010 & & 2242 & 2220 & 2 \\
\hline$u=$ & 232 & 000 & 1958 & ODS & $02 / 08 / 65$ & 136.000 & 0.8794 & 113.03547 & 74.74 & 794.60 & 65834 & 53641 & 0,0511 & 2030 & 2010 & 2 \\
\hline u. & 232 & 000 & 1958 & ODS & $02 / 08 / 65$ & 138,000 & 0.7939 & 109.78282 & 34.18 & 1405.92 & 66277 & 48752 & 0,0 & 1989 & 1969 & ? \\
\hline u. & 232 & 000 & 1958 & ODS & $02 / 08 / 65$ & 140.000 & 0,8118 & 106 & 121. & 1252 & 66725 & 501 & 0,0 & 1677 & 1660 & 2 \\
\hline U. & 232 & 000 & 1958 & ODS & $02 / 08 / 65$ & 142,000 & 0.9004 & 103,68498 & 60. & 620.69 & 66406 & 55 & 0,0 & 18 & 17 & 2 \\
\hline u- & 232 & 000 & 1958 & ODS & $02 / 08 / 65$ & 144,000 & 0,9595 & 100.82485 & 24.03 & 241,27 & 64661 & 57485 & 0.0541 & 1622 & 1606 & 2 \\
\hline 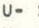 & 232 & 000 & 1958 & ODS & $02 / 08 / 65$ & 146.000 & 0.9751 & 98,08144 & 14.66 & 145.21 & 66050 & 59672 & 0,0548 & 1499 & 1484 & 2 \\
\hline $\begin{array}{l}u= \\
u=\end{array}$ & $\begin{array}{l}232 \\
232\end{array}$ & $\begin{array}{l}000 \\
000\end{array}$ & $\begin{array}{l}1958 \\
1958\end{array}$ & $\begin{array}{l}\text { ODS } \\
\text { ODS }\end{array}$ & $\begin{array}{l}02 / 08 / 65 \\
02 / 08 / 65\end{array}$ & $\begin{array}{l}148,000 \\
150.000\end{array}$ & $\begin{array}{l}0,9662 \\
0,9805\end{array}$ & $\begin{array}{l}95.44850 \\
92.92018\end{array}$ & $\begin{array}{l}19,99 \\
11,47\end{array}$ & $\begin{array}{l}195,28 \\
110.55\end{array}$ & $\begin{array}{l}65842 \\
60656\end{array}$ & $\begin{array}{l}58941 \\
55100\end{array}$ & $\begin{array}{l}0,0556 \\
0,0563\end{array}$ & $\begin{array}{l}1459 \\
1440\end{array}$ & $\begin{array}{l}1445 \\
1425\end{array}$ & $\frac{2}{2}$ \\
\hline 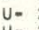 & 232 & 000 & 1958 & ODS & $02 / 08 / 65$ & & & & 12. & & & & & & & 2 \\
\hline$U=$ & 232 & 000 & 1958 & ODS & $02 / 08$ & & & & & & & & & & & 2 \\
\hline U. & 232 & 000 & 1958 & ODS & $02 / 08 / 65$ & 1 & 0 & .90993 & 21 & 202 & 62 & & 0,0 & 12 & 1277 & 2 \\
\hline U. & 232 & 000 & 1958 & ODS & $02 / c$ & & & & 14 & 136. & 64271 & & & 1183 & 1171 & 2 \\
\hline . & 232 & 000 & 1958 & ODS & $02 / 08 / 65$ & 160.000 & 0.95 & 81 & & 215. & & 59138 & & 11 & & 2 \\
\hline ve & 232 & 000 & 1958 & ODS & $02 / 08 / 65$ & 162,000 & 0.93 & 79.66407 & & 348.63 & 67508 & 58477 & 0.06 & 1189 & 1177 & 3 \\
\hline U. & 232 & 000 & 1958 & ODS & $02 / 08 / 65$ & 164,000 & 0.8635 & $77,73.290$ & 85,30 & 752.05 & 67016 & 53611 & 0.0616 & 1119 & 1108 & 2 \\
\hline J. & 232 & 000 & 1458 & ODS & 0 & 166.000 & 0.6724 & 75,87110 & 230.76 & 20 & 66 & 41 & & 11 & & 2 \\
\hline $\mathrm{J}$ & 232 & 000 & 19 & ODS & & & & & 218 & 188 & & & & & & 2 \\
\hline u. & 232 & 000 & 1958 & ODS & $02 / 08 / 65$ & 170.000 & 0,8819 & 72 & 73 & 621.24 & 67 & 552 & 0.0639 & 1135 & 1124 & 2 \\
\hline u. & 232 & 000 & 1958 & UDS & $02 / 08 /$ & 172.000 & 0.95 & 6 & & & & & & 11 & 1178 & 2 \\
\hline u. & 232 & 000 & 1958 & ODS & $02 / 08 / 65$ & 174.000 & 0.96 & 69.05 & 23 & 194. & 65 & 58 & 0,0 & 1120 & 1109 & 2 \\
\hline$u=$ & 232 & 000 & 1958 & ODS & & & & & & 122.37 & 61 & 55 & & 12 & & 2 \\
\hline u- & 232 & 000 & 1958 & ODS & $02 / 08 / 65$ & 178,000 & 0.9760 & 65,98611 & & 114 & 64 & 58 & & 1152 & 1141 & 2 \\
\hline$U=$ & 232 & 000 & 1958 & ODS & $02 / 08 / 65$ & 180.000 & 0,9700 & 64.52790 & 17.7 & 142.26 & 65664 & 590 & 0,0 & 1184 & 1172 & $=$ \\
\hline$u=$ & 232 & 000 & 1958 & ODS & $02 / 08 / 65$ & .00 & 0,975 & 8 & 14,4 & 114.99 & 66266 & 59873 & 0,0684 & 1127 & 1115 & 2 \\
\hline U. & 32 & 000 & 1950 & ODS & $02 / 08 / 65$ & 184,000 & 0,9682 & 61,75284 & 18.76 & 147,40 & 66680 & 59804 & 0.0691 & 1203 & 1191 & ? \\
\hline
\end{tabular}




\begin{tabular}{|c|}
\hline IDENT \\
\hline$u=232$ \\
\hline$U=232$ \\
\hline$U=232$ \\
\hline$U=232$ \\
\hline$u=232$ \\
\hline $\begin{array}{l}u=232 \\
u=232\end{array}$ \\
\hline$u=232$ \\
\hline$u=232$ \\
\hline$U=232$ \\
\hline$v=232$ \\
\hline U. 232 \\
\hline $\begin{array}{l}U= \\
u=232 \\
U=232\end{array}$ \\
\hline $\begin{array}{l}u=232 \\
u= \\
232\end{array}$ \\
\hline$u=232$ \\
\hline U. 232 \\
\hline$U=232$ \\
\hline$u=232$ \\
\hline$u=232$ \\
\hline$u=232$ \\
\hline$v=232$ \\
\hline$u=232$ \\
\hline$u=232$ \\
\hline$u=232$ \\
\hline$u=232$ \\
\hline$u=232$ \\
\hline$u-232$ \\
\hline$u=232$ \\
\hline$u=232$ \\
\hline$U=232$ \\
\hline$U=232$ \\
\hline$U=232$ \\
\hline$u=232$ \\
\hline$v=232$ \\
\hline$v=232$ \\
\hline$u=232$ \\
\hline U. 232 \\
\hline$u=232$ \\
\hline$u=232$ \\
\hline$u=232$ \\
\hline$u=232$ \\
\hline$U=232$ \\
\hline$U=232$ \\
\hline$u=232$ \\
\hline$u=232$ \\
\hline$u=232$ \\
\hline$u=232$ \\
\hline$v=232$ \\
\hline$v=232$ \\
\hline$u=232$ \\
\hline$U=232$ \\
\hline$u=232$ \\
\hline$v=232$ \\
\hline$v=232$ \\
\hline$v=232$ \\
\hline U. $23 ?$ \\
\hline$v=232$ \\
\hline$u=232$ \\
\hline$v=232$ \\
\hline$v=232$ \\
\hline$u=232$ \\
\hline$U=232$ \\
\hline$U=232$ \\
\hline$U=232$ \\
\hline$u=232$ \\
\hline$U=232$ \\
\hline$U=232$ \\
\hline U- 232 \\
\hline$U=232$ \\
\hline$v=232$ \\
\hline$v=232$ \\
\hline$v=232$ \\
\hline$U=232$ \\
\hline$U=232$ \\
\hline$v=232$ \\
\hline$U=232$ \\
\hline$u=232$ \\
\hline$U=232$ \\
\hline$v=232$ \\
\hline$u=232$ \\
\hline$y=232$ \\
\hline \\
\hline $\begin{array}{l}U=232 \\
U=232\end{array}$ \\
\hline $\begin{array}{l}u=232 \\
U=232\end{array}$ \\
\hline u. 232 \\
\hline U. 232 \\
\hline$v=232$ \\
\hline$u=232$ \\
\hline U. 232 \\
\hline U. 232 \\
\hline$U=232$ \\
\hline$U=232$ \\
\hline$v=232$ \\
\hline U. 232 \\
\hline$u=232$ \\
\hline U. 232 \\
\hline$u=232$ \\
\hline$v=232$ \\
\hline
\end{tabular}




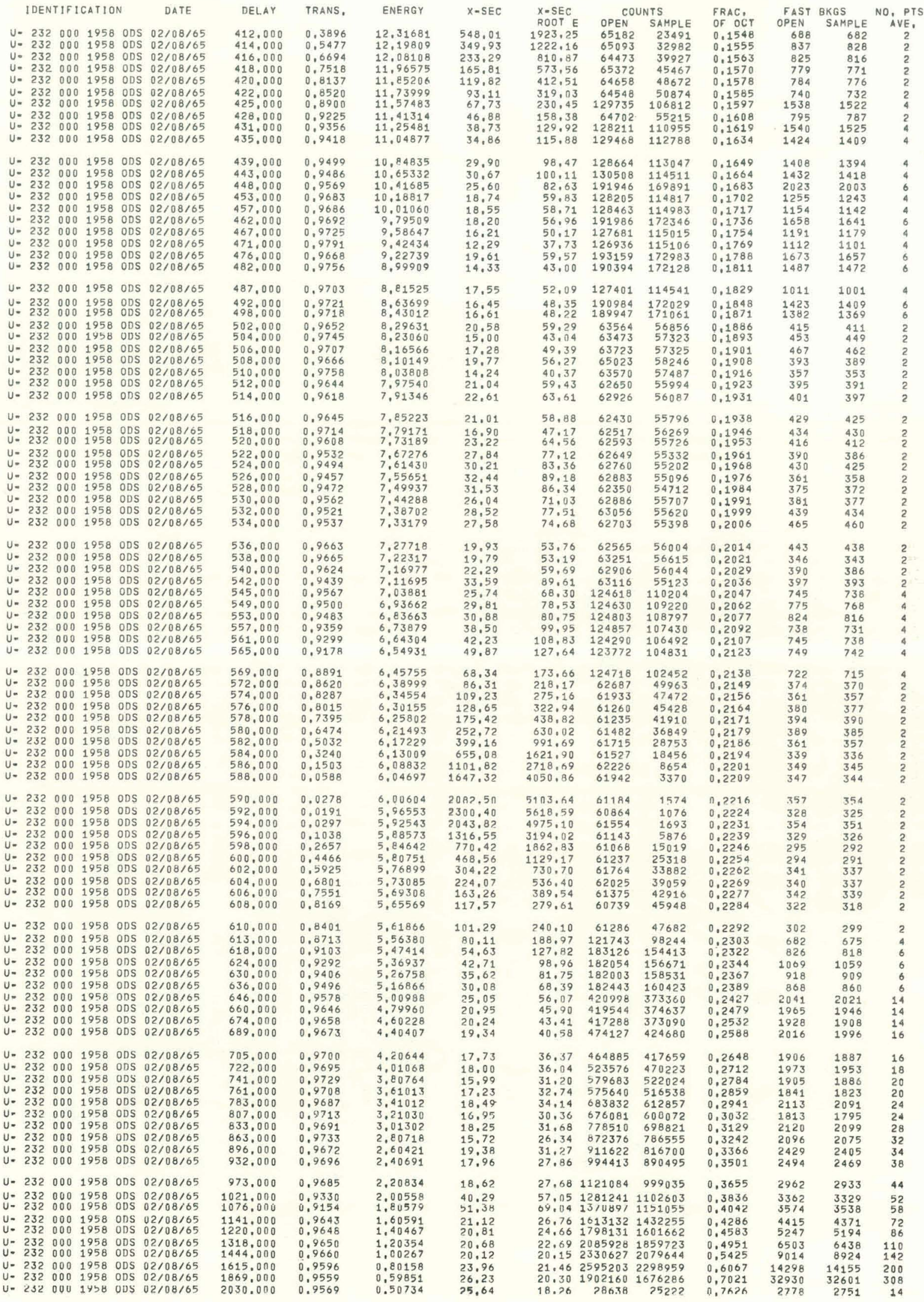


RUN 1959

\begin{tabular}{|c|c|c|c|c|c|c|c|c|c|c|c|c|c|c|c|}
\hline IDENT & fific & CATION & & DATE & DEL,AY & TRANS, & ENERGY & $X$-SEC & $\mathrm{X}=\mathrm{SEC}$ & $=0$ & & FRAC, & FAST & BKGS & NO, PTS \\
\hline$u=232$ & 000 & 1959 & $S$ & $02 / 08 / 65$ & 39.000 & .9566 & 1374,55884 & 24,87 & & $\begin{array}{l}\text { OPEN } \\
76232\end{array}$ & & Of oct & $\begin{array}{l}\text { OPEN } \\
8705\end{array}$ & SAMPLE & \\
\hline 5 & 000 & 1 & ODS & 02 & 41.000 & 0,9580 & 1243.72635 & 24.05 & 848,25 & 74313 & 67834 & 0.0167 & 7628 & 7551 & $\frac{2}{2}$ \\
\hline $\begin{array}{l}232 \\
232\end{array}$ & 000 & & ODS & $\begin{array}{l}02 / 08 / 65 \\
02 / 108 / 65\end{array}$ & 43.000 & 0.9509 & 1130.72147 & 28.21 & 948.47 & 69245 & 62740 & & 8449 & 8365 & 2 \\
\hline & 000 & 1959 & ODS & $\begin{array}{l}02 / 08 / 65 \\
02 / 08 / 65\end{array}$ & 45,000 & 0.9550 & 1032.44641 & 25.82 & 829.53 & $77^{1441.3}$ & 64980 & 0.0184 & 7040 & 6969 & 2 \\
\hline & $\begin{array}{l}000 \\
000\end{array}$ & $\begin{array}{l}1959 \\
1959\end{array}$ & $\begin{array}{l}\text { ODS } \\
\text { ODS }\end{array}$ & $\begin{array}{l}02 / 08 / 65 \\
02 / 08 / 65\end{array}$ & $\begin{array}{l}47,000 \\
49,000\end{array}$ & $\begin{array}{l}0.9588 \\
0,9730\end{array}$ & $\begin{array}{l}946 ., 44817 \\
870,76385\end{array}$ & $\begin{array}{l}23.60 \\
15,36\end{array}$ & $\begin{array}{l}726,19 \\
453,33\end{array}$ & $\begin{array}{l}73103 \\
72453\end{array}$ & $\begin{array}{l}\begin{array}{l}66779 \\
67165\end{array} \\
6\end{array}$ & $\begin{array}{l}0.0192 \\
0,0200\end{array}$ & $\begin{array}{l}6587 \\
6162\end{array}$ & $\begin{array}{l}6521 \\
6100\end{array}$ & ${ }_{2}^{2}$ \\
\hline & 000 & 1959 & ODS & $08 / 65$ & .000 & .9597 & 803.80776 & 23.06 & 653.85 & 73162 & 66896 & 0,0208 & 5602 & 5546 & $\begin{array}{l}2 \\
2\end{array}$ \\
\hline & 000 & 1959 & ODS & 165 & on & 9485 & 8764 & & 27 & 73203 & & 16 & 4933 & 4884 & $\begin{array}{l}2 \\
2\end{array}$ \\
\hline & 000 & 1959 & ODS & $08 / 65$ & .000 & .9528 & 691,14182 & 7 & 713,28 & 72369 & 6569 & .0225 & 4859 & 4811 & \\
\hline 32 & 000 & 1959 & ODS & $02 / 08 / 65$ & 57.000 & 0.9400 & 643,49153 & 34.70 & 880.18 & 73701 & 66002 & 0.0233 & 4459 & 4414 & 2 \\
\hline $\begin{array}{l}u=232 \\
4.233\end{array}$ & 000 & 1959 & ODS & $02 / 08 / 65$ & 59.000 & 0.9446 & 600,60442 & 31.98 & 783.67 & 72962 & 65657 & 0.0241 & 4646 & 4599 & 2 \\
\hline $\begin{array}{l}u=232 \\
u=232 \\
u\end{array}$ & $\begin{array}{l}000 \\
000\end{array}$ & $\begin{array}{l}1959 \\
1959\end{array}$ & $\begin{array}{l}\text { ODS } \\
\text { ODS }\end{array}$ & $\begin{array}{l}02 / 08 / 15 \\
02 / 08 / 65\end{array}$ & $\begin{array}{l}61.000 \\
63,000\end{array}$ & $\begin{array}{l}0.9528 \\
0.9520\end{array}$ & $\begin{array}{l}561.86616 \\
526,75838\end{array}$ & $\begin{aligned} 27708 \\
27,58\end{aligned}$ & $\begin{array}{l}641,94 \\
633,04\end{array}$ & $\begin{array}{l}72645 \\
72081\end{array}$ & $\begin{array}{l}65944 \\
65373\end{array}$ & $\begin{array}{l}0.0249 \\
0.0257\end{array}$ & $\begin{array}{l}4204 \\
3562\end{array}$ & $\begin{array}{l}4162 \\
3526\end{array}$ & $\frac{2}{2}$ \\
\hline$U=232$ & 000 & 1959 & ODS & $02 / 08 / 65$ & 65.000 & 0.9437 & 494.84118 & 32.48 & 722.51 & 70608 & 63479 & 0.0265 & 3647 & 3610 & 2 \\
\hline$U=232$ & 000 & 1959 & ODS & $02 / 08 / 65$ & 67.000 & 0.9458 & $\begin{array}{r}465.73936 \\
\end{array}$ & 31.25 & 674,51 & $69377 ?$ & 62508 & 0.0274 & 3548 & 3513 & 2 \\
\hline $\begin{array}{l}=232 \\
u-232\end{array}$ & $\begin{array}{l}000 \\
000\end{array}$ & $\begin{array}{l}1959 \\
1959\end{array}$ & $\begin{array}{l}\text { ODS } \\
\text { ODS }\end{array}$ & $02 / 08 / 65$ & $\begin{array}{l}69.000 \\
71.000\end{array}$ & $\begin{array}{l}0.9254 \\
0.9377\end{array}$ & $\begin{array}{l}439,13127 \\
414,73993\end{array}$ & $\begin{array}{l}43.49 \\
36.06\end{array}$ & $\begin{array}{l}911.38 \\
734.44\end{array}$ & $\begin{array}{l}67773 \\
62670\end{array}$ & $\begin{array}{l}59743 \\
55981\end{array}$ & $\begin{array}{l}0.0282 \\
0.0290\end{array}$ & $\begin{array}{l}3352 \\
3145\end{array}$ & $\begin{array}{l}3318 \\
3114\end{array}$ & ${ }_{2}^{2}$ \\
\hline & 000 & $\begin{array}{l}1959 \\
1959\end{array}$ & ODS & $02 / 08 / 65$ & 73,000 & 0.9442 & 392,32576 & $\begin{array}{l}36.06 \\
32.17\end{array}$ & $\begin{array}{l}734,44 \\
637.15\end{array}$ & 56285 & $\begin{array}{l}23981 \\
50628\end{array}$ & 0.0298 & $\begin{array}{l}3145 \\
3039\end{array}$ & $\begin{array}{l}3114 \\
3008\end{array}$ & 2 \\
\hline & & & ODS & $02 / 08 / 65$ & 75.0 & tere & 71 & . & 538.12 & 48265 & 43744 & .0306 & $3=27$ & 3290 & \\
\hline$u-232$ & 000 & 1959 & ODS & $02 / 08 / 65$ & 77.000 & 0.9358 & 352.62337 & 37.20 & 698.49 & 30941 & 27581 & 0.0314 & 3709 & 3731 & \\
\hline$v-232$ & 000 & 1959 & ODS & $02 / 08 / 65$ & 79.000 & 0.9233 & 334.99503 & 44,76 & 819.24 & 28307 & 24895 & 0,0323 & 3592 & 3556 & ? \\
\hline $\begin{array}{l}y=232 \\
y=232 \\
y=23\end{array}$ & $\begin{array}{l}000 \\
000\end{array}$ & $\begin{array}{l}1959 \\
1959\end{array}$ & $\begin{array}{l}0 D S \\
0 D S\end{array}$ & $\begin{array}{l}02 / 08 / 65 \\
02108665\end{array}$ & $\begin{array}{l}81 ; 000 \\
83,000\end{array}$ & $\begin{array}{l}0.9404 \\
0.9436\end{array}$ & $\begin{array}{r}318,65630 \\
303 ; 48439\end{array}$ & $\begin{array}{l}34: 48 \\
32: 55\end{array}$ & $\begin{array}{l}615: 43 \\
567: 10\end{array}$ & $\begin{array}{l}43314 \\
57457\end{array}$ & $\begin{array}{l}38798 \\
51643\end{array}$ & $\begin{array}{l}0.0331 \\
0.0339\end{array}$ & $\begin{array}{l}\begin{array}{l}4161 \\
4321\end{array} \\
\end{array}$ & $\begin{array}{l}4120 \\
4278\end{array}$ & $\frac{2}{2}$ \\
\hline$u=232$ & 000 & 1959 & ODS & $02 / 08 / 65$ & 85.000 & 0.9461 & 289,37079 & 31.04 & 528,09 & 63696 & 57404 & 0.0347 & 4601 & 4615 & $=$ \\
\hline 32 & . & 1959 & 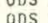 & $02 / 08 / 65$ & 89,000 & 0.9114 & 263. & (51.09 & $\begin{array}{l}010.49 \\
844.57\end{array}$ & $\begin{array}{l}68318 \\
68318\end{array}$ & 59311 & 0,0363 & 5 & $\begin{array}{l}5249 \\
5434\end{array}$ & \\
\hline & 000 & 1959 & $00 \mathrm{~S}$ & $02 / 08 / 65$ & 91,000 & 0.8980 & 252.46999 & 0 & 958,74 & 71110 & 60820 & 0.0372 & 5648 & $\begin{array}{l}5434 \\
5592\end{array}$ & 2 \\
\hline & 000 & 1959 & ODS & $02 / 08 / 05$ & 93,000 & 0.9387 & 241. & 35.44 & 551.03 & 69896 & 62496 & 0.0380 & 5804 & 5746 & 2 \\
\hline 32 & 000 & 1959 & ODS & $02 / 08 / 65$ & 95.000 & 0.9643 & 231.65695 & 20.41 & 310.60 & 68411 & 62830 & 0.0388 & 5743 & 5685 & \\
\hline$v=232$ & 000 & 1959 & ODS & $02 / 08 / 65$ & 97.000 & 0.9540 & 222.20257 & 26.41 & 393,65 & 68716 & 62438 & 0.0396 & 5674 & 5617 & 2 \\
\hline 32 & 000 & 1959 & ODS & $02 / 08 / 65$ & 99.000 & 0.9278 & 213,31537 & 42,00 & 613,42 & 70482 & 62285 & 0.0404 & 6000 & 5940 & \\
\hline $\begin{array}{l}u=232 \\
u=232\end{array}$ & 000 & $\begin{array}{l}1959 \\
1959\end{array}$ & ODS & 3165 & 101.000 & 0.9234 & & & 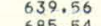 & 71178 & 62599 & 412 & 5930 & 5871 & \\
\hline & 000 & 1959 & ons & $02 / 08 / 65$ & .000 & 0.8658 & 189.63301 & $\begin{array}{l}48.8 \\
80.7\end{array}$ & $\begin{array}{r}585.54 \\
1112.05\end{array}$ & 70528 & $\begin{array}{l}60911 \\
58160\end{array}$ & $\begin{array}{l}0.0421 \\
0.0429\end{array}$ & 87 & $\begin{array}{l}3861 \\
5432\end{array}$ & $=$ \\
\hline 32 & 000 & 1959 & ODS & $02 / 08 / 65$ & 107,000 & 0,9060 & 182,61018 & 55.34 & 747.88 & 71679 & 61849 & 0,0437 & 5487 & 5432 & 2 \\
\hline & 000 & 1959 & ODS & $02 / 08 / 65$ & 109,000 & 0,9381 & 175 . & 35 & 475.11 & 71530 & 03908 & 0.0445 & 5380 & 5326 & 2 \\
\hline & 000 & 1959 & ODS & $02 / 08 / 65$ & 111,000 & 0,9418 & 169.68623 & 33.60 & 437.64 & 69900 & 62699 & 0.0453 & 5324 & 5271 & ? \\
\hline 32 & 000 & 1959 & ODS & $02 / 08 / 65$ & 113.000 & 0.9302 & 163.7 & 40.58 & 519.23 & 68922 & 61055 & 0.0461 & 4719 & 4672 & 2 \\
\hline $\begin{array}{l}u=232 \\
u=232\end{array}$ & $\begin{array}{l}000 \\
000\end{array}$ & $\begin{array}{l}1959 \\
1959\end{array}$ & $\begin{array}{l}\text { ODS } \\
\text { ODS }\end{array}$ & $\begin{array}{l}02 / 08 / 65 \\
02 / 08 / 65\end{array}$ & 117.000 & $\begin{array}{l}0.8792 \\
0.9037\end{array}$ & $\begin{array}{l}1558.08726 \\
152,72876\end{array}$ & $\begin{array}{l}72.17 \\
56.79\end{array}$ & $\begin{array}{l}907,40 \\
701.79\end{array}$ & $\begin{array}{l}70259 \\
69599\end{array}$ & $\begin{array}{l}58829 \\
59896\end{array}$ & $\begin{array}{l}0,0470 \\
0,0478\end{array}$ & $\begin{array}{l}4693 \\
4102\end{array}$ & $\begin{array}{l}4646 \\
4061\end{array}$ & 2 \\
\hline & 000 & & & $02 / 08 / 6$ & & & & & & & & & Floc & 7001 & \\
\hline & 000 & 1959 & ODS & & & 8507 & & 90. & $\begin{array}{r}1083.18 \\
108\end{array}$ & 65922 & 53406 & 0.0494 & $\begin{array}{l}3684 \\
3631\end{array}$ & $\begin{array}{l}3944 \\
3594\end{array}$ & ${ }^{2}$ \\
\hline & 000 & 1959 & ODS & 021 & 123.000 & 0.9249 & 138.19182 & 43. & 514,23 & 63069 & 55552 & 0,0502 & 3367 & 3333 & 2 \\
\hline & 000 & 1959 & ODS & $02 / 08 /$ & 25.000 & 0,9003 & 133.80506 & 58. & 680.73 & 59720 & 51203 & 0.0510 & 3028 & 2998 & 2 \\
\hline & 000 & $\begin{array}{l}1959 \\
1950\end{array}$ & ODS & $02 / 0$ & 127.000 & 0.8165 & 129.62391 & 113.64 & 1293.83 & 60985 & & 0,0519 & 2620 & 2594 & 2 \\
\hline & 000 & $\begin{array}{l}1959 \\
1950\end{array}$ & (2) & 027 & 9.000 & 0.8812 & 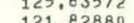 & 70. & $\begin{array}{l}794,44 \\
311,92\end{array}$ & $\begin{array}{l}62739 \\
61473\end{array}$ & $\begin{array}{l}52649 \\
55660\end{array}$ & $\begin{array}{r}0.0527 \\
0.0535\end{array}$ & $\begin{array}{l}2257 \\
2357\end{array}$ & 2234 & 2 \\
\hline & 000 & 1959 & ODS & $02 / 08$ & 133,000 & 0.9452 & $118,1923 ?$ & 31.5 & $\begin{array}{l}311,92 \\
343.35\end{array}$ & $\begin{array}{l}65756 \\
65756\end{array}$ & 59186 & 0,0543 & 2050 & 2030 & \\
\hline$u=2$ & 000 & 1959 & ODS & $02 / 08$ & 135.000 & 0.8981 & 114.71627 & 60.24 & 645.25 & 69987 & 59853 & 0.0551 & 1881 & 1863 & \\
\hline$u=232$ & 000 & 1959 & ODS & $02 / 08 / 65$ & 137,000 & 0,8451 & 111,39134 & 94,34 & 995,66 & 70100 & 56412 & 0.0560 & 1876 & 1857 & \\
\hline$v=232$ & 000 & 1959 & ODS & $02 / 08 / 6$ & 139.000 & 0.7752 & 108.20889 & 142 & 1485.17 & 70028 & 51 & 0.0568 & 1801 & 1783 & 2 \\
\hline & 000 & 1 & ODS & & & 51 & 91 & & 967 & & & 0310 & 1559 & 1543 & \\
\hline 2 & 000 & $\begin{array}{l}1959 \\
1959\end{array}$ & $\begin{array}{l}0 D S \\
0 D S\end{array}$ & $02 / 08$ & 143.000 & $\begin{array}{l}0.9204 \\
0.9625\end{array}$ & $\begin{array}{r}102.23991 \\
90.43895\end{array}$ & 46. & $\begin{array}{l}470.45 \\
213.74\end{array}$ & $\begin{array}{l}70 \\
68\end{array}$ & & & 1536 & 1521 & ? \\
\hline & 000 . & 1959 & 005 & 021 & 7.000 & 0.9772 & $\begin{array}{l}35154 \\
5154\end{array}$ & 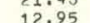 & 127.36 & 70 & & 0.0592 & & & \\
\hline & 000 & 1959 & ODS & $02 / 0$ & ano & 0.9792 & $94>2$ & 11 & 114, & 68 & 64032 & 0.00 & 1412 & & \\
\hline & 000 & 1959 & ODS & $02 / 0$ & 11.000 & 0.9790 & 91.69352 & 11. & 108.87 & 59809 & 558 & $00,08-2$ & 1492 & 1477 & \\
\hline & 000 & 1959 & ODS & 021 & 153.000 & $0.97 \mathrm{C}$ & 8 & 14 & 13 & & & & & & \\
\hline$u-232$ & 000 & 1959 & ODS & $02 / 08$ & 155.000 & 0.9785 & 87.02202 & 12.2 & 113.80 & 65082 & 60631 & 0.0633 & 1402 & 1388 & 2 \\
\hline u. 232 & 000 & 1959 & ODS & $02 / 08 / 65$ & 157.000 & 0.9695 & 84,81902 & 17,35 & 159,83 & 66942 & 61793 & 0,0641 & 1359 & 1326 & 2 \\
\hline 32 & 000 & 1959 & ODS & 021 & 9.0 & 0.9 & 4 & 25 & 230 & 70 & 64 & 0.6 & 1311 & 1298 & \\
\hline $\begin{array}{l}u=232 \\
u=232 \\
u=23\end{array}$ & $\begin{array}{l}000 \\
000\end{array}$ & $\begin{array}{l}1959 \\
1959\end{array}$ & ODS & $\begin{array}{l}02 / 08 \\
02 / 08\end{array}$ & $\begin{array}{l}161 ; 000 \\
163 ; 000\end{array}$ & $\begin{array}{l}0.9581 \\
0.9362\end{array}$ & $\begin{array}{r}80,65676 \\
78.68960\end{array}$ & $\begin{array}{l}\begin{array}{l}24.01 \\
36.94\end{array} \\
36\end{array}$ & $\begin{array}{l}215.59 \\
327.67\end{array}$ & $\begin{array}{l}70719 \\
71183\end{array}$ & $\begin{array}{l}64508 \\
63450\end{array}$ & $\begin{array}{l}0.0658 \\
0.0666\end{array}$ & 1324 & 1311 & 2 \\
\hline u. 232 & 000 & 1959 & ODS & $02 / 08 / 65$ & 165.000 & 0.7933 & 76,79354 & 129 & 1137.44 & 70966 & 53 & 0,0 & 6 & 1461 & 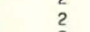 \\
\hline & 000 & 19 & ODS & 021 & 0 & 0.6 & & 278 & & 71508 & & & 1353 & 1340 & 2 \\
\hline 2 & $\begin{array}{l}000 \\
000\end{array}$ & $\begin{array}{l}1959 \\
1959\end{array}$ & ODS & $\begin{array}{l}02 / 108, \\
02 / 08\end{array}$ & $\begin{array}{l}169.000 \\
171,000\end{array}$ & $\begin{array}{l}0.1610 \\
0.9300\end{array}$ & $\begin{array}{l}73,20136 \\
71,49906\end{array}$ & $\begin{array}{r}153 \\
40\end{array}$ & $\begin{array}{r}1309,90 \\
3443,77\end{array}$ & $\begin{array}{l}710 \\
716\end{array}$ & $\begin{array}{l}51451 \\
63403\end{array}$ & $\begin{array}{l}0.0690 \\
0.0698\end{array}$ & $\begin{array}{l}1415 \\
1440\end{array}$ & $\begin{array}{l}1401 \\
1426\end{array}$ & $?$ \\
\hline & 000 & 1959 & ODS & 02 & 3,000 & 0,9571 & ? & $24,6 \quad>$ & 205,60 & 71065 & 64750 & 7 & 5 & 1 & \\
\hline & 000 & 1959 & $O D$ & 021 & & & & $27+2$ & & & & & & & \\
\hline U. 232 & 000 & 1959 & ODS & $02 / 08$ & 177.000 & 0.9558 & 66.73382 & 25.34 & 206.98 & 66191 & 60229 & 0,0723 & 1626 & 1610 & ? \\
\hline$U=232$ & 000 & 1959 & ODS & $02 / 08 / 65$ & 179,000 & 0.9701 & 65.25090 & 16.99 & 137.24 & 70149 & 64707 & 0.0731 & 1681 & 1664 & 2 \\
\hline $\begin{array}{l}u=232 \\
u=232 \\
u\end{array}$ & $\begin{array}{l}000 \\
000\end{array}$ & $\begin{array}{l}195 \\
195 \\
-19\end{array}$ & $\begin{array}{l}\text { ODS } \\
\text { ODS }\end{array}$ & $\begin{array}{l}02 / 08 / 65 \\
02 / 108 / 65\end{array}$ & $\begin{array}{l}181: 000 \\
183: 000\end{array}$ & $\begin{array}{l}0,9798 \\
0,9729\end{array}$ & $\begin{array}{r}63,81685 \\
62,42957\end{array}$ & $\begin{array}{l}11,44 \\
15 ; 37\end{array}$ & $\begin{array}{r}91: 35 \\
121 ; 48\end{array}$ & $\begin{array}{l}70043 \\
70799\end{array}$ & $\begin{array}{l}65533 \\
65575\end{array}$ & $\begin{array}{l}0.0739 \\
0: 0747\end{array}$ & $\begin{array}{l}1543 \\
1577\end{array}$ & $\begin{array}{l}1527 \\
1561\end{array}$ & $?_{2}^{2}$ \\
\hline & 000 & 1959 & ODS & & tor.uo & , & & & 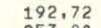 & 104 & & & & 13 & \\
\hline & 000 & 1959 & ODS & & $187.0 \quad-$ & 0.9 & & & & & & & 1674 & 1657 & \\
\hline$y$ & 000 & 1959 & ODS & 021 & 189,000 & 0.9 & & 18.3 & 3 & & & 0.013 & & $\begin{array}{l}1660 \\
1583\end{array}$ & \\
\hline & 000 & & Do & & & & & & & & & & & & \\
\hline 32 & 000 & 1959 & 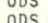 & $\begin{array}{l}021 \\
021\end{array}$ & & & & . & & & 65 & 0.0 & & $\begin{array}{l}1636 \\
1515\end{array}$ & \\
\hline$u=232$ & 000 & 1959 & ODS & $02 / 08 / 65$ & 197,000 & 0,9155 & 53,87163 & 49.50 & 363,33 & 71495 & 62303 & 0.0805 & $\begin{array}{l}1501 \\
1538\end{array}$ & $\begin{array}{l}1513 \\
1523\end{array}$ & \\
\hline$u$ & 000 & 1959 & ODS & $02 / 0$ & 9. & 0.8 & 52.7 & & 57 & 71344 & 5 & 3 & 8 & 1443 & \\
\hline & 000 & 1959 & ODS & & & & & & & & & & & & \\
\hline & 000 & 1959 & ons & & & 0.96 & & & & & & & & 1. & \\
\hline$y$ & 000 & $\begin{array}{l}1959 \\
1959\end{array}$ & $\begin{array}{l}\text { ODS } \\
\text { ODS }\end{array}$ & & 100 & 0.96 & & & & & & & & & \\
\hline 23 & . & & (D) & & & 0.90 & & & & & & & & & \\
\hline u. & 000 & 1959 & $D S$ & & 21 & $0:-2-1$ & & & & & & & 13 & & \\
\hline 232 & 000 & 1959 & DDS & & 213.0 & 0,95 & toct & & 179.09 & & & & 1293 & 1280 & \\
\hline $\begin{array}{l}232 \\
232\end{array}$ & 000 & & & 508165 & 215.000 & 0.9523 & 5.22886 & 16 & 184.17 & 69613 & 63098 & 0.0878 & 228 & 1216 & \\
\hline 232 & 000 & 1959 & DS & $02 / 08 / 65$ & $21 \% .000$ & 0.8714 & 44.39899 & 7.16 & 514.16 & 69439 & 57591 & 0.0886 & 1253 & 1240 & \\
\hline
\end{tabular}




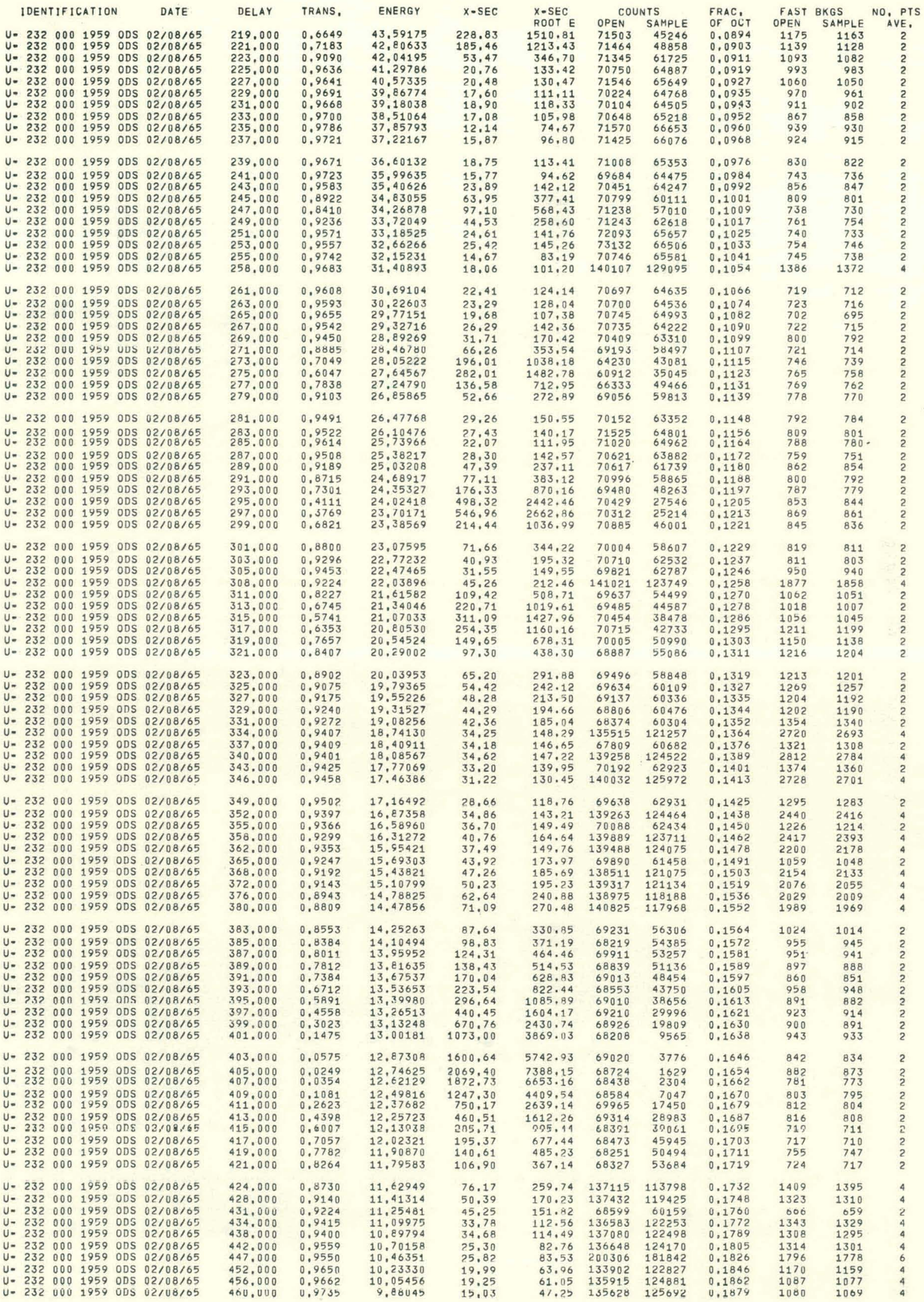




\begin{tabular}{|c|c|c|c|c|c|c|c|c|c|c|c|c|c|c|c|}
\hline IDENT & IC & CATION & & DATE & DELAY & TRANS. & ENERGY & $X$-SEC & $x=$ SEC & COL & IS & FRAC, & FAST & 8KGS & No, PT \\
\hline-232 & 000 & 1959 & ODS & $02 / 08 / 65$ & 465.000 & 0.9676 & 9,6691 & 18,45 & 57.38 & 202661 & 186694 & 0.1899 & 1637 & 1621 & \\
\hline - 232 & 000 & 1959 & ODS & $02 / 081$ & 470.000 & 0.9765 & 9.46448 & 13.32 & 40.98 & 134784 & 125295 & 0.1919 & 1015 & 1005 & \\
\hline 232 & 000 & 1959 & ODS & $02 / 0$ & $\begin{array}{l}475.000 \\
481.000\end{array}$ & 0.9730 & 9,26 & 15.32 & $\begin{array}{l}46.64 \\
50.06\end{array}$ & $\begin{array}{l}203061 \\
202904\end{array}$ & $\begin{array}{l}188098 \\
187484\end{array}$ & 400 & $\begin{array}{l}1554 \\
1435\end{array}$ & 1538 & \\
\hline $\begin{array}{l}232 \\
232\end{array}$ & $\begin{array}{l}000 \\
000\end{array}$ & $\begin{array}{l}1959 \\
1959\end{array}$ & $\begin{array}{l}\text { ODS } \\
\text { ODS }\end{array}$ & $\begin{array}{l}02 / 08 / 65 \\
02 / 08 / 65\end{array}$ & $\begin{array}{l}481.000 \\
486.000\end{array}$ & $\begin{array}{l}0.970 \text { ? } \\
0.9781\end{array}$ & $\begin{array}{l}9.03654 \\
8.85156\end{array}$ & $\begin{array}{l}16.95 \\
12.44\end{array}$ & $\begin{array}{l}50,96 \\
37,02\end{array}$ & $\begin{array}{l}202904 \\
134252\end{array}$ & $\begin{array}{l}187484 \\
125056\end{array}$ & $\begin{array}{l}0,1964 \\
0,1985\end{array}$ & $\begin{array}{r}1435 \\
986\end{array}$ & $\begin{array}{r}1420 \\
976\end{array}$ & \\
\hline U. 232 & 000 & 1959 & ODS & $02 / 08 / 65$ & 491.000 & 0.9701 & 8,67221 & 17.03 & 50.15 & 202540 & 187125 & 0.2005 & 1457 & 1442 & \\
\hline $\begin{array}{l}232 \\
232\end{array}$ & 000 & 1959 & ODS & 02108 & 497.000 & 0.9800 & 8,46408 & 11.32 & 32.92 & $\begin{array}{r}200234 \\
66693\end{array}$ & 186903 & 0.2030 & 1331 & 1317 & \\
\hline$=\begin{array}{l}232 \\
232\end{array}$ & $\begin{array}{ll}000 \\
000\end{array}$ & $\begin{array}{l}1959 \\
1959\end{array}$ & $\begin{array}{l}\text { ODS } \\
\text { ODS }\end{array}$ & $\begin{array}{l}02108\} \\
02 / 081\end{array}$ & $\begin{array}{l}501: \\
503:\end{array}$ & $\begin{array}{l}0.9747 \\
0.9700\end{array}$ & $\begin{array}{l}8.32946 \\
8,26336\end{array}$ & $\begin{array}{l}14: 34 \\
17,08\end{array}$ & $\begin{array}{l}41,39 \\
49,09\end{array}$ & $\begin{array}{l}66693 \\
67008\end{array}$ & ${ }_{11}^{21}$ & ${ }_{4}^{6}$ & $\begin{array}{l}455 \\
452\end{array}$ & 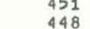 & \\
\hline$u=232$ & & & $\begin{array}{l}\text { ODS } \\
\text { ODS }\end{array}$ & $02 / 08 / 65$ & $\begin{array}{l}503.000 \\
505,000\end{array}$ & 0,9744 & $\begin{array}{l}8.26336 \\
8.19804\end{array}$ & $\begin{array}{l}11.08 \\
14.53\end{array}$ & $\begin{array}{l}49,09 \\
41,60\end{array}$ & 67240 & $\begin{array}{l}61911 \\
62409\end{array}$ & $\begin{array}{l}0.2054 \\
0.2062\end{array}$ & $\begin{array}{l}452 \\
450\end{array}$ & $\begin{array}{l}448 \\
446\end{array}$ & \\
\hline 232 & 000 & 1959 & ODS & $02 / 08 / 65$ & 507.000 & 0.9731 & 8,13348 & 15 & 7 & 67 & 6272 & 0.2071 & 436 & 432 & \\
\hline $\begin{array}{l}232 \\
232\end{array}$ & 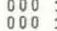 & $\begin{array}{l}\begin{array}{l}1959 \\
1959\end{array} \\
195\end{array}$ & $\begin{array}{l}\text { ODS } \\
\text { ODS }\end{array}$ & $\begin{array}{l}02 / 0865 \\
02 / 08 / 65\end{array}$ & $\begin{array}{l}509.000 \\
511: 000\end{array}$ & $\begin{array}{l}0.9659 \\
0.9707\end{array}$ & $\begin{array}{l}8.06969 \\
8 ; 00665\end{array}$ & $\begin{array}{l}19.44 \\
16: 65\end{array}$ & $\begin{array}{l}\begin{array}{r}55.21 \\
47: 11\end{array}\end{array}$ & $\begin{array}{l}68867 \\
66500\end{array}$ & $\begin{array}{l}63179 \\
61571 \\
61571\end{array}$ & $\begin{array}{l}0,2079 \\
0,2087\end{array}$ & $\begin{array}{l}450 \\
453\end{array}$ & $\begin{array}{l}446 \\
448\end{array}$ & \\
\hline 232 & 000 & 1959 & ODS & $02 / 08 / 65$ & 513.000 & 0.9717 & 7.94434 & 16.10 & 45.37 & 65579 & 60694 & 0.2095 & 400 & 396 & \\
\hline $2^{2}$ & 000 & 1959 & ODS & 021 & $\begin{array}{l}515.4 \\
517.4\end{array}$ & 0. & & & 83 & 63 & 9 & & 425 & 420 & \\
\hline 232 & 000 & 1959 & ODS & $02 / 08 / 6$ & 519.000 & 0.9678 & $7: 76172$ & $\begin{array}{l}21.47 \\
18.33\end{array}$ & 51.08 & $\begin{array}{l}60581 \\
66581\end{array}$ & $\begin{array}{l}61370 \\
61370\end{array}$ & 10 & $\begin{array}{l}445 \\
453\end{array}$ & $\begin{array}{l}\begin{array}{l}441 \\
448\end{array} \\
4\end{array}$ & \\
\hline & 000 & 1959 & ODS & $02 / 08 / 65$ & 521.000 & 0.9691 & 7,70224 & 17.58 & 48.80 & 65826 & 60753 & 0.2128 & 411 & 407 & \\
\hline $\begin{array}{l}u=232 \\
u=232\end{array}$ & & & $\begin{array}{l}\text { ODS } \\
\text { ODS }\end{array}$ & $\begin{array}{l}02 / 08 / 65 \\
02 / 08 / 65\end{array}$ & $\begin{array}{l}\begin{array}{l}523.000 \\
525,000\end{array} \\
525,00\end{array}$ & $\begin{array}{l}0,9504 \\
0.9495\end{array}$ & $\begin{array}{l}7.64345 \\
7.58532\end{array}$ & $\begin{array}{l}28.51 \\
29.08\end{array}$ & $\begin{array}{l}78,82 \\
80,08\end{array}$ & $\begin{array}{l}66276 \\
65902\end{array}$ & $\begin{array}{l}59985 \\
59583\end{array}$ & $\begin{array}{l}0,2136 \\
0,2144\end{array}$ & $\begin{array}{l}461 \\
392\end{array}$ & $\begin{array}{l}456 \\
389\end{array}$ & \\
\hline 232 & 000 & 1959 & ODS & $02 / 08 / 65$ & 527.000 & 0,9413 & 7,52786 & 33.89 & 93.00 & 66194 & 59332 & 0,2152 & 434 & 429 & \\
\hline 23 & 00 & $\begin{array}{l}1959 \\
1959\end{array}$ & on & $02 / 08 / 65$ & 529.000 & $\begin{array}{l}0.9531 \\
0.9604\end{array}$ & 7.47104 & 26.91 & 73.56 & 65734 & 59657 & 0.2160 & 491 & 486 & \\
\hline & & & & & & 0.9534 & 7.35933 & 26,78 & 72.65 & & & 7 & & & \\
\hline & 000 & 1959 & ODS & $02 / 08 / 65$ & 535,000 & 0.9567 & 7.30441 & 24.84 & 67.14 & 66341 & 60433 & 0.2185 & 354 & 351 & \\
\hline & 000 & 9 & $0 x+2>$ & $02 / 08$ & 37.000 & 0.9645 & .25010 & 20.27 & 54,58 & 65750 & 60371 & $160+20$ & 459 & 454 & \\
\hline & 000 & 10 & & $02 / 0$ & 985 & & 9640 & & 52.51 & & & & & 457 & \\
\hline $\begin{array}{l}232 \\
232\end{array}$ & $\begin{array}{l}000 \\
000\end{array}$ & $\begin{array}{l}1959 \\
1959\end{array}$ & $\begin{array}{l}\text { ODS } \\
\text { ODS }\end{array}$ & $\begin{array}{l}02 / 08 / 65 \\
02 / 108 / 65\end{array}$ & 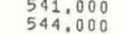 & $\begin{array}{l}0.7502 \\
0.9588\end{array}$ & $\begin{array}{l}7,14329 \\
7: 06472\end{array}$ & $\begin{array}{l}28.61 \\
23.58\end{array}$ & $\begin{array}{l}76,48 \\
62.67\end{array}$ & $\begin{array}{l}66990 \\
132262\end{array}$ & $\begin{array}{r}00554 \\
120524\end{array}$ & $\begin{array}{l}0.2209 \\
0.2222\end{array}$ & $\begin{array}{l}415 \\
810\end{array}$ & $\begin{array}{l}{ }_{411}^{812} \\
802\end{array}$ & \\
\hline$u-232$ & 000 & 1959 & ODS & $02 / 08 / 65$ & 547,000 & 0.9537 & 6.98744 & 26.55 & 70.18 & 66092 & 59831 & 0,2234 & 476 & 472 & \\
\hline $\begin{array}{l}232 \\
232\end{array}$ & 000 & 1959 & ODS & $02 / 081$ & 550.000 & 0.9454 & 6.91142 & 31. & 82,82 & 131830 & 118058 & 0,2246 & 833 & 825 & \\
\hline & . & ? & & & & & 15 & & $\begin{array}{r}89.72 \\
103.52\end{array}$ & $\begin{array}{l}197802 \\
130822\end{array}$ & $\begin{array}{l}75644 \\
15256\end{array}$ & & $\begin{array}{l}1229 \\
818\end{array}$ & $\begin{aligned} 1216 \\
810\end{aligned}$ & \\
\hline 232 & 000 & 1959 & ODS & 02108 &, 000 & 0.9160 & 6 & 49 & 126.09 & 130823 & 113608 & 3 & & 827 & \\
\hline 232 & 000 & 1959 & ODS & $02 / 08$ & 568. & $0.8 \varepsilon$ & 1 & & & 130337 & 110 & & & 783 & \\
\hline & $\begin{array}{l}000 \\
000\end{array}$ & $\begin{array}{l}1959 \\
1959\end{array}$ & & $\begin{array}{l}021 \\
0211\end{array}$ & . 571. & $\begin{array}{l}0.8 \\
0.8\end{array}$ & 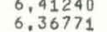 & $\begin{array}{l}78 \\
95 \\
95\end{array}$ & 年 & $\begin{array}{l}66 \\
66\end{array}$ & $\begin{array}{l}54597 \\
53406\end{array}$ & & $\begin{array}{l}335 \\
378\end{array}$ & $\begin{array}{l}331 \\
375\end{array}$ & \\
\hline-5 & 0003 & $\begin{array}{l}1959 \\
1959\end{array}$ & 0 & $02 / 08$ & 5. & 0.79 & 32 & 126 & 317.61 & 65 & & & 36 & 363 & \\
\hline $\begin{array}{l}4=3 \\
4=\end{array}$ & 000 & 19 & & & & & & & & & & & & 355 & \\
\hline$u=232$ & & 1959 & ODS & $02 / 08$ & 579.000 & 0.6873 & .23642 & 210,21 & 524.97 & 65365 & 42741 & 0,2365 & 41 & 414 & \\
\hline 232 & 000 & 1959 & ons & $02 / 08 /$ & 10 & 0.5 & 6 & 30 & 757.91 & & 35 & & 369 & 365 & \\
\hline & 000 & 1959 & & & & & & & & & & & & & \\
\hline & 0003 & $\begin{array}{l}1959 \\
1959\end{array}$ & ODS & & 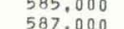 & & & & & & & & & & \\
\hline & 0001 & 1959 & oos & & 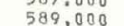 & . & . & & & & & & & & \\
\hline & 000 & 1959 & & $02 / 0$ & & & 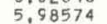 & & & & & & & & \\
\hline & 0003 & 1959 & & 021 & & & & & & & & & & & \\
\hline & 0003 & 1959 & & 021 & 6 & 0.05 & & 0 & & & & & & 357 & \\
\hline 2 & 0001 & 1959 & OD & $02 / 0$ & 59 & 0,1 & 86 & 1017 & 45 & 64 & 10026 & & & 37 & \\
\hline U. 232 & 0003 & 1959 & ODS & $02 / 08$ & 599.0 & 0.33 & .82 & 611.53 & 1476.18 & 644 & 20603 & 0.2 & 387 & 383 & \\
\hline & 000 & 1959 & ODS & $02 / 08 / 65$ & 601.000 & 0,5030 & 5.78820 & 385.21 & 926.76 & 64513 & 30878 & 0,2454 & 318 & 315 & \\
\hline the & $\begin{array}{l}000 \\
000\end{array}$ & $\begin{array}{l}1959 \\
1959\end{array}$ & 00 & & & & & & & & & & & & \\
\hline ? & 000 & 1959 & ODS & & & & & & & & & & & & \\
\hline ? & 0001 & 1959 & ODS & & & & & 113 & & & & & & & \\
\hline 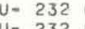 & 000 & 1959 & ot & & & & & & & & & & & & \\
\hline 2 & 8003 & 1959 & ${ }_{0}^{O D}$ & $02 / 4$ & & 0.9 & & & & & & & & & \\
\hline & 000 & $\begin{array}{l}1959 \\
1959\end{array}$ & & & & 0.92 & 5 & & $\begin{array}{r}104.78 \\
72,71\end{array}$ & & & & & & \\
\hline 2: & 000 & 1959 & OD & 021 & 635. & 0.9 & & 31 & 71.58 & 192 & 1.77 & & 900 & 891 & \\
\hline & 000 & 1959 & 0 & 02 & 641.000 & 544 & .08 & 26. & 99 & 18 & 17 & & 853 & 844 & \\
\hline & & & & & & & & & & & & & & & \\
\hline & 0 & & & & & & & & & & & & & & \\
\hline t & 000 & $\begin{array}{l}1959 \\
1959\end{array}$ & $O D$ & & & & & & & & & & & & \\
\hline ? & 000 & 1959 & ons & & & & & & & & & & & & \\
\hline & 0001 & 1959 & ODS & & & & & & & & & & & & \\
\hline & 00 & $\begin{array}{l}1959 \\
959\end{array}$ & $\begin{array}{c}0.05 \\
005\end{array}$ & $02 / 0$ & 74 & & & & $\begin{array}{l}29.58 \\
28.55\end{array}$ & & $\begin{array}{l}611 \\
550\end{array}$ & & & & 0 \\
\hline . & 000 & $\begin{array}{l}2959 \\
1959\end{array}$ & ODS & $02 / 08$, & 792,000 & 0.9722 & 3.33305 & $\begin{array}{l}15.20 \\
15.79\end{array}$ & $\begin{array}{l}28,85 \\
28,82\end{array}$ & $\begin{array}{l}793429 \\
704268\end{array}$ & 651358 & 0.3235 & 2063 & $\begin{array}{l}1608 \\
2042\end{array}$ & 24 \\
\hline 232 & 000 & 1959 & 00 & 021 & 7.000 & 0.9728 & 3219 & 15.4 & 27.34 & 75 & & & 2076 & 2055 & \\
\hline & 00 & 198 & & & & & & & & & & & & & $\begin{array}{l}30 \\
32\end{array}$ \\
\hline 23 & $\begin{array}{l}000 \\
000 \\
0\end{array}$ & $\begin{array}{l}1959 \\
1959\end{array}$ & $\begin{array}{l}O D S S \\
O D S\end{array}$ & & & & & & & & & & & & \\
\hline & 000 & 1959 & of & 02108 & 948,0 & $\begin{array}{l}0.9686 \\
0\end{array}$ & 5 & $\begin{array}{l}16 \\
17\end{array}$ & 年 & & $\begin{array}{l}904674 \\
965744\end{array}$ & & 29 & & \\
\hline & 00 & 195 & or & & & & & & & & & & & & \\
\hline & 000 & 1959 & ODS & & & & & & & & & & & & \\
\hline & $\begin{array}{l}000 \\
000\end{array}$ & & 然 & & & & & & & & & & & & \\
\hline & 000 & & ODS & $08 / 65$ & 1257.000 & 0.9661 & 32319 & 19.32 & 22.2 & $2<0$ & 699476 & .5134 & 6901 & 6832 & \\
\hline & 000 & & & & & & & & & & & & & & 122 \\
\hline & & & & & & & & & & & & & & 136 & \\
\hline & & & & & 1 & & & 2.02 & 6.49 & 8855 & 004 & $\begin{array}{l}.07886 \\
.7886\end{array}$ & 2746 & 72019 & \\
\hline
\end{tabular}


RUN 1960

\begin{tabular}{|c|c|c|c|c|c|c|c|c|c|c|c|c|c|c|c|}
\hline IDENT & iffic & EATION & & DATE & DELAY & TRANS. & ENERGY & $X$ XEEC & $\begin{array}{l}X-S E C \\
\text { BOOT } E\end{array}$ & $\begin{array}{cc}c C \\
O P E N\end{array}$ & 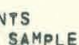 & $\begin{array}{l}\text { FRAC, } \\
\text { OP OCF }\end{array}$ & $\begin{array}{c}\text { FAST } \\
\text { OPEN }\end{array}$ & $\begin{array}{l}\text { BKGS } \\
\text { SAMPLE }\end{array}$ & NO PTS \\
\hline U. 232 & 000 & 1960 & ODS & $02 / 08 / 65$ & 15.000 & 0,9260 & 9292,01770 & 22,09 & $129,44^{2}$ & $\begin{array}{l}15358 \\
1535\end{array}$ & $\begin{array}{l}\text { SAMPLE } \\
13_{144}\end{array}$ & 0,0120 & 4308 & $\begin{array}{l}\text { SAAPLE } \\
42222\end{array}$ & AVE, \\
\hline U. 232 & 000 & 1960 & ODS & $02 / 08 / 65$ & 16.000 & 0.9241 & 8166,81250 & 22.67 & 048,32 & 13546 & 11570 & 0.0128 & 4401 & 4313 & 2 \\
\hline $\begin{array}{l}y=232 \\
y=233\end{array}$ & 0.00 & 1960 & ODS & $02 / 08 / 65$ & 17.000 & 0.9336 & 7234,26984 & 19.73 & 677,76 & 14124 & 12187 & 0.0136 & 3075 & 3014 & 2 \\
\hline $\begin{array}{l}u=232 \\
u \cdot \\
u=232\end{array}$ & 000 & 1960 & $\begin{array}{l}\text { ODS } \\
0.55\end{array}$ & $\begin{array}{l}02 / 08 / 65 \\
02 / 08 / 65\end{array}$ & 18.000 & $\begin{array}{l}0.9256 \\
0.9431\end{array}$ & $\begin{array}{r}6452.79010 \\
5791.42377\end{array}$ & $\begin{array}{l}22.19 \\
16.81\end{array}$ & $\begin{array}{l}782,86 \\
279,52\end{array}$ & $\begin{array}{l}13117 \\
14344\end{array}$ & 11221 & 0.0144 & 2077 & 3022 & $2^{2}$ \\
\hline & & & & $\begin{array}{l}02108 / 15 \\
02 / 08 / 65\end{array}$ & & 0,9491 & 5226,75995 & $\begin{array}{l}16.81 \\
15.02\end{array}$ & $\begin{array}{l}27,52,68 \\
085,68\end{array}$ & $\begin{array}{l}14344 \\
14727\end{array}$ & 12916 & 0.0160 & 2346 & 2299 & $\frac{2}{2}$ \\
\hline$U=232$ & 000 & 1960 & ODS & $02 / 08 / 65$ & 21.000 & 0,9283 & 4740,82538 & 21.36 & 470,49 & 15137 & 12986 & 0,0168 & 1745 & 1710 & 2 \\
\hline & & & ODS & $02 / 08 / 65$ & 22,000 & 0,9308 & 4319,63635 & 20,60 & 354.02 & 14984 & 12888 & 0.0176 & 1437 & 1408 & \\
\hline - 232 & 000 & 1960 & ODS & $02 / 08 / 65$ & 23.000 & 0,9315 & 3952.18146 & 20.37 & 280.31 & 15194 & 13080 & 0.0184 & 1063 & 1042 & $\varepsilon$ \\
\hline - 232 & 000 & 1960 & ODS & $02 / 08 / 65$ & 24.000 & 0.9179 & 3629.69443 & 24.62 & 483.14 & 15070 & 12782 & 0.0192 & 1501 & 1471 & 2 \\
\hline $\begin{array}{l}232 \\
232\end{array}$ & 000 & 1960 & ODS & $02 / 08 / 65$ & 25.000 & 0.9433 & 3345.12637 & 16.77 & $\begin{array}{l}970.20 \\
3313.57\end{array}$ & $\begin{array}{l}14428 \\
15326\end{array}$ & $\begin{array}{l}12576 \\
13043\end{array}$ & $\begin{array}{l}0.0200 \\
0.0208\end{array}$ & $\begin{array}{r}1687 \\
833\end{array}$ & $\begin{array}{r}1653 \\
817\end{array}$ & $=$ \\
\hline $\begin{array}{l}232 \\
232\end{array}$ & 000 & 1960 & $\begin{array}{l}\text { ODS } \\
\text { ODS }\end{array}$ & $\begin{array}{l}02 / 08 / 65 \\
02 / 08 / 65\end{array}$ & $\begin{array}{l}26,000 \\
27,000\end{array}$ & $\begin{array}{l}0.9210 \\
0.9316\end{array}$ & $\begin{array}{r}3092,75739 \\
2867.90671\end{array}$ & $\begin{aligned} 23.62 \\
20.35\end{aligned}$ & 089.65 & 13404 & 11539 & 0.0216 & 1460 & 1639 & \\
\hline & 000 & 1960 & ODS & $02 / 08 / 65$ & 28,000 & 0.9406 & $2666^{\circ}, 71426$ & 97.57 & (907.57 & 12734 & 111068 & 0.024 & 1384 & 1357 & \\
\hline 200 & 000 & 1960 & ODS & $02 / 08 / 65$ & 20.000 & 0.9095 & 2485,9738 ? & 27.23 & 357,61 & 13014 & 10937 & 0232 & 926 & 907 & \\
\hline 232 & 000 & 1960 & ODS & $02 / 08 / 65$ & 30.000 & 0.9240 & 2323,00443 & 22.71 & 094,35 & 12419 & 10603 & 0.0240 & 1279 & 1253 & 2 \\
\hline U. 232 & 000 & 1960 & ODS & $02 / 08 / 65$ & 31.000 & 0.9104 & 2175,55045 & 26.95 & 257,15 & 14020 & 11793 & 0.0248 & 615 & 602 & 2 \\
\hline & 000 & 1960 & ODS & $02 / 08 / 65$ & 32.000 & 0.9195 & 2041.70313 & 24.11 & 089.45 & 14383 & 12219 & 0256 & 694 & 680 & 2 \\
\hline $\begin{array}{l}y=233 \\
y=232 \\
y=23\end{array}$ & $\begin{array}{l}000 \\
000\end{array}$ & $\begin{array}{l}1960 \\
1960\end{array}$ & $\begin{array}{l}0 D S \\
\text { ODS }\end{array}$ & $02 / 08 / 65$ & $\begin{array}{l}33.000 \\
34: 000\end{array}$ & $\begin{array}{l}0.9225 \\
0.9274\end{array}$ & $\begin{array}{r}1919,868 \\
1808^{\circ} 5646\end{array}$ & $\begin{array}{l}23.18 \\
21: 65\end{array}$ & $\begin{array}{l}013,47 \\
920 ; 52\end{array}$ & $\begin{array}{l}14486 \\
14447\end{array}$ & $\begin{array}{l}2334 ! \\
12379\end{array}$ & : & 810 & $\begin{array}{l}894 \\
7994\end{array}$ & \\
\hline U. 232 & 000 & 1960 & ODS & $02 / 08 / 65$ & 35.000 & 0.9328 & 1706.69713 & 19.97 & 825,08 & 14735 & 12699 & 0280 & 514 & 504 & \\
\hline$U=232$ & 000 & 1960 & ODS & $02 / 08 / 65$ & 36.000 & 9129 & 1613,19753 & 26.18 & 051,49 & 14904 & 12570 & 288 & 486 & 476 & \\
\hline 232 & 000 & 1960 & ODS & $02 / 08 / 65$ & 37.000 & 0.9020 & 1527.17604 & 29.64 & 158.12 & 14538 & 12115 & 0296 & 725 & 710 & \\
\hline 232 & 000 & 1960 & ODS & $02 / 08 / 65$ & 38.000 & 0,9218 & 1447.85594 & 23.39 & 889,88 & 14126 & 12030 & 0304 & 760 & 745 & \\
\hline 232 & 000 & 1960 & ODS & $02 / 08 / 65$ & 39,000 & 0,9186 & 1374,55884 & & 904,47 & 14368 & 12193 & 0,0312 & 746 & 731 & \\
\hline 10 232 & 000 & 1960 & ODS & $02 / 08 / 65$ & 10.00 & .9184 & 1306,68990 & 4. & 883,88 & 13706 & 11629 & .0320 & 1124 & 1101 & 2 \\
\hline$U=232$ & 000 & 1960 & ODS & $02 / 08 / 65$ & 41.000 & 0.9119 & 1243,72635 & 26.50 & 934,51 & 13469 & 11346 & 0.0328 & 1025 & 1005 & 2 \\
\hline & 000 & 1960 & ODS & $02 / 08 / 65$ & 42.000 & 0.9229 & 1185,20634 & 23.05 & 793,71 & 12538 & 10689 & 0.0336 & 1327 & 2300 & \\
\hline U. 232 & 000 & 1960 & ODS & $02 / 08 / 65$ & 43.000 & 0.8908 & 1130.72147 & 33.19 & 116.21 & 12323 & 10141 & 344 & 1319 & 1293 & 2 \\
\hline $\begin{array}{l}y=232 \\
y=233\end{array}$ & 000 & 1960 & ODS & $02 / 08 / 65$ & $\begin{array}{l}44.000 \\
45 .\end{array}$ & 0.9084 & 1079,90909 & $\begin{array}{r}27.59 \\
26 .\end{array}$ & 906.76 & 13041 & 10943 & $\begin{array}{l}0.0352 \\
0.0360\end{array}$ & $\begin{array}{l}1166 \\
1515\end{array}$ & $\begin{array}{l}1143 \\
1485\end{array}$ & 2 \\
\hline $\begin{array}{l}232 \\
232\end{array}$ & 000 & 1960 & ODS & 02108165 & $\begin{array}{l}45,000 \\
46.000\end{array}$ & $\begin{array}{l}0,9126 \\
0,9080\end{array}$ & $\begin{array}{r}1032,44641 \\
988.04536\end{array}$ & 26.27 & 871,68 & 13360 & 11206 & 0.0368 & 1601 & $\begin{array}{l}1403 \\
1569\end{array}$ & \\
\hline $\begin{array}{l}232 \\
232\end{array}$ & 000 & 1900 & uns & 02708153 & 40.000 & 0.9000 & 900.04300 & 270 & 721.00 & 13600 & 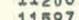 & 0.0376 & 2001 & 1400 & \\
\hline $\begin{array}{l}232 \\
232\end{array}$ & 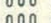 & 1960 & ODS & $02 / 08 / 65$ & 48,000 & 0.9305 & 907,42361 & 20. & 623,39 & 13474 & 11581 & 34 & 1448 & 1419 & \\
\hline 23 ? & 000 & 1960 & ODS & 年 & 49 & 0.92 & 870 & & & 13164 & 11 & 2 & 1729 & $3_{4}$ & \\
\hline 232 & 000 & 1960 & ODS & $02 / 08 / 65$ & 50.000 & 0.9145 & 836,28150 & 25.68 & 742,74 & 13333 & 11262 & 0.0400 & 1602 & 1570 & 2 \\
\hline $\begin{array}{l}U=232 \\
U_{0}\end{array}$ & 000 & 1960 & ODS & $02 / 08 / 65$ & 51.000 & 0.9277 & 803,80776 & 21.56 & 611,21 & $\begin{array}{l}13164 \\
43265\end{array}$ & 11280 & 0.0408 & 1493 & 1463 & $2_{2}^{2}$ \\
\hline $\begin{array}{l}0.032 \\
U .232\end{array}$ & 000 & $\begin{array}{l}1900 \\
1960\end{array}$ & ODS & 021 & $\begin{array}{l}52.000 \\
53.000\end{array}$ & $\begin{array}{l}0.9053 \\
0.8920\end{array}$ & 744 & $\begin{array}{l}28.57 \\
32.83\end{array}$ & $\begin{array}{l}794.49 \\
895,55\end{array}$ & $\begin{array}{l}13265 \\
13348\end{array}$ & $\begin{array}{l}12093 \\
1099\end{array}$ & 0 & $\begin{array}{l}1431 \\
1452\end{array}$ & $\begin{array}{l}1428 \\
1423\end{array}$ & $2_{2}^{2}$ \\
\hline U. 232 & 000 & 1960 & ODS & 02108165 & 54.000 & 0.9099 & 716.97668 & 27.11 & & 13210 & & 0.0432 & 1500 & 1479 & 2 \\
\hline U. 232 & 000 & 1960 & ODS & 0210 & 55.000 & 0,9114 & 691 & & 70 & 1 & & 0. & 1308 & 1282 & 2 \\
\hline 232 & 000 & 1960 & ODS & $02 / 08 / 65$ & 56.000 & 0.8816 & $\begin{array}{l}666.67857 \\
643\end{array}$ & 36.19 & 934.45 & 13497 & $\begin{array}{l}10990 \\
10338\end{array}$ & $\begin{array}{l}0.0448 \\
0.0456\end{array}$ & $\begin{array}{l}1220 \\
1540\end{array}$ & $\begin{array}{l}1195 \\
1511\end{array}$ & 2 \\
\hline $\begin{array}{l}232 \\
232\end{array}$ & 000 & 1960 & 005 & 02108165 & $\begin{array}{l}57.000 \\
58.000\end{array}$ & 0.8886 & 621,49345 & $33 ., 91$ & 828,41 & $\begin{array}{l}12040 \\
12989\end{array}$ & 10679 & $\begin{array}{l}0,0430 \\
0,0464\end{array}$ & 1217 & $\begin{array}{l}1911 \\
192\end{array}$ & \\
\hline 202 & 000 & 1960 & ODS & $02 / 08 / 65$ & 59.0 & 0.8990 & 600 , & & 749.14 & 12698 & & 2 & 1157 & 1134 & \\
\hline U. 232 & $\begin{array}{l}000 \\
000\end{array}$ & $\begin{array}{l}1960 \\
1960\end{array}$ & ODS & $02 / 08 / 65$ & 60,000 & 0.9037 & 580,75111 & 29.09 & 701.15 & 12376 & $\begin{array}{l}10329 \\
00\end{array}$ & 0.0480 & 1246 & 1221 & $\frac{2}{2}$ \\
\hline$U=232$ & 000 & 1960 & ODS & $02 / 08 / 65$ & 61,000 & 0,9246 & 501,80616 & 22,52 & 533.83 & 12500 & 10679 & 0 . & 2087 & 1066 & 2 \\
\hline 32 & 000 & 1960 & ODS & & 62 & 0,9121 & & & 530.25 & 12563 & & 86 & 1016 & 996 & \\
\hline $\begin{array}{l}y=232 \\
y=232\end{array}$ & $\begin{array}{l}000 \\
000\end{array}$ & $\begin{array}{l}1960 \\
1960\end{array}$ & $\begin{array}{l}\text { ODS } \\
\text { ODS }\end{array}$ & $\begin{array}{l}02 / 0 \\
02 / 10\end{array}$ & $\begin{array}{l}63.000 \\
64.000\end{array}$ & $\begin{array}{l}0.9277 \\
0.8830\end{array}$ & $\begin{array}{l}526,75 \\
510,42\end{array}$ & $\begin{array}{l}21 \\
35\end{array}$ & $\begin{array}{l}5 \\
5\end{array}$ & $\begin{array}{l}125 \\
127\end{array}$ & $\begin{array}{l}10 \\
10\end{array}$ & $\begin{array}{l}0.0504 \\
0.0512\end{array}$ & 810 & 794 & \\
\hline U. 232 & 000 & 1960 & ODS & $02 / 08 / 65$ & 65.000 & 0,8961 & 494,84 & 31.52 & & 12455 & & & 713 & 699 & 2 \\
\hline$y=232$ & 000 & 1960 & ODS & 0210 & 66.000 & 0,9070 & 479 & & & 12422 & 10404 & & & 650 & 2 \\
\hline $\begin{array}{l}232 \\
232\end{array}$ & 000 & 1960 & ODS & $02 / 08 / 65$ & 67,000 & 0,9027 & $\begin{array}{l}465,73936 \\
452\end{array}$ & 29.40 & 634,55 & $\begin{array}{l}12489 \\
12293\end{array}$ & $\begin{array}{l}10411 \\
9952\end{array}$ & $\begin{array}{l}0,0536 \\
0.0544\end{array}$ & $\begin{array}{l}479 \\
566\end{array}$ & $\begin{array}{l}462 \\
555\end{array}$ & 2 \\
\hline $\begin{array}{l}=.232 \\
U=232\end{array}$ & 000 & $\begin{array}{l}1960 \\
1960\end{array}$ & ODS & . $02 / 08$ & 69.000 & 0.8708 & $\begin{array}{r}422.14160 \\
439.13127\end{array}$ & 37 & 862.65 & 12134 & 9708 & 0,050 & & 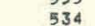 & \\
\hline$U=232$ & 000 & $\begin{array}{l}1960 \\
1960\end{array}$ & ODS & $02 / 08 / 65$ & 70.000 & 0,8784 & 426.67428 & $\begin{array}{l}41 \\
37\end{array}$ & 769.14 & 12002 & 9735 & 0.0560 & $=$ & 530 & \\
\hline 232 & 000 & 1960 & ODS & $02108 /$ & 71.000 & 0 & .735 & 32 & 661. & 11841 & 9765 & 0.0568 & 466 & 456 & 2 \\
\hline & 000 & 1960 & ODS & & & & & & & & & 0. & & 494 & \\
\hline y. 232 & 000 & 1960 & ODS & & 73. & 0. & 392 & & & & & & & 409 & 2 \\
\hline 232 & 000 & 1960 & ODS & $02 / 6$ & & & & & & & & 0. & & $\begin{array}{l}350 \\
465\end{array}$ & 2 \\
\hline $\begin{array}{l}232 \\
232\end{array}$ & 000 & 1960 & ODS & $02 / 08 / 65$ & 75.000 & 0.9489 & $\begin{array}{l}371.68071 \\
369\end{array}$ & 15.06 & $\begin{array}{l}290.30 \\
367.79\end{array}$ & $\begin{array}{l}9393 \\
7964\end{array}$ & $\begin{array}{l}82 \\
68 \\
68\end{array}$ & $\begin{array}{l}0.0600 \\
0.0608\end{array}$ & $\begin{array}{l}474 \\
398\end{array}$ & $\begin{array}{l}465 \\
390\end{array}$ & $\frac{2}{2}$ \\
\hline 232 & 000 & 1960 & 005 & 021 & 78 & $\begin{array}{l}0.9369 \\
0.8607\end{array}$ & $361,9 t$ & & & I & & & & 441 & \\
\hline 232 & 000 & 年 1700 & ODS & 年 & & 0.6 & 3 & & & 47 & & & & 445 & \\
\hline & 008 & 1960 & 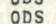 & & & & & & & & & & & & \\
\hline 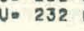 & 000 & 1960 & ODS & $02 / 08$ & 80.000 & 0.8755 & 326,67250 & 38.20 & 69 & 7497 & 6060 & 0. & 443 & 43 & 2 \\
\hline$U=232$ & 000 & 1960 & ODS & $02 / 0$ & 81,000 & 0.8504 & 318,65630 & 46.55 & 830,88 & 9110 & 7153 & 0.0648 & 443 & 434 & 2 \\
\hline 232 & 000 & 1960 & ODS & & & & & & & & & & 395 & 387 & 2 \\
\hline$y=:$ & 000 & $\begin{array}{l}1940 \\
1960\end{array}$ & nins & & $\begin{array}{l}A 3 . \\
84 .\end{array}$ & 0.8 & ? & & & 20 & & & & 374 & \\
\hline U. 232 & 000 & 1960 & ODS & & 85,000 & 0,9225 & & & & & & & 348 & 341 & 2 \\
\hline$y=232$ & 000 & 1960 & ODS & & 86 & 0.8 & & & & 11 & 96 & & 533 & 522 & 2 \\
\hline 232 & 000 & 1960 & ODS & $02 / 0$ & 87.000 & 0.8993 & 276.21 & & 506 & & & 0.0 & 8 & 370 & 2 \\
\hline 238 & 000 & 1960 & uns & 0210 & 88.000 & 0.8903 & 209 & 33.38 & $54 e^{2}-1$ & 11705 & $\begin{array}{l}9620 \\
8092\end{array}$ & 0.078 & 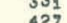 & 年25 & 2 \\
\hline 32 & $\begin{array}{l}000 \\
000\end{array}$ & $\begin{array}{l}1960 \\
1960\end{array}$ & $\begin{array}{l}\text { ODS } \\
\text { ODS }\end{array}$ & $02 / 08 / 65$ & $\begin{array}{l}89.000 \\
90,000\end{array}$ & $\begin{array}{l}0.8202 \\
0.7456\end{array}$ & 258,11160 & $\begin{array}{l}54.32 \\
84.32\end{array}$ & 354.67 & 11829 & 8142 & 0,0720 & 417 & $400^{\circ}$ & $\frac{2}{2}$ \\
\hline 232 & $\mathrm{nOC}$ & 1960 & DDS & 021 & 9 & 0 & 25 & 3 & 863 & 11 & & & 454 & 445 & ? \\
\hline & & & & & & & & & & & & & & 400 & \\
\hline & 000 & 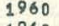 & ODS & & & & & & & & & & & $\begin{array}{l}488 \\
455\end{array}$ & \\
\hline $\begin{array}{l}232 \\
232\end{array}$ & 0 & 0 & $\begin{array}{l}\text { ODS } \\
\text { ODS }\end{array}$ & 02 & & & 2 & & & & 10 & & 6 & 5 & ${ }_{2}^{2}$ \\
\hline 232 & 000 & 1960 & ODS & & 96, & & 6,25 & & $\begin{array}{l}170 \\
467\end{array}$ & 11 & gह & & 507 & 497 & \\
\hline $\begin{array}{l}232 \\
232\end{array}$ & 000 & $\begin{array}{l}1960 \\
1960\end{array}$ & ODS & $02 / 08 / 65$ & 97. & 0,9052 & 222,20 & & 426 & & & & 372 & 364 & $=$ \\
\hline 32 & 00 & 0 & $\begin{array}{l}\text { ons } \\
\text { ons }\end{array}$ & $02 / 08 / 65$ & $98: 0$ & 0 & 2 & 36 & & & 93 & $0:$ & $\begin{array}{l}362 \\
477\end{array}$ & $\begin{array}{l}551 \\
467\end{array}$ & $2_{2}^{2}$ \\
\hline $\begin{array}{l}u=232 \\
u=232 \\
U=23\end{array}$ & $\begin{array}{l}000 \\
000\end{array}$ & $\begin{array}{l}1960 \\
1960\end{array}$ & $\begin{array}{l}\text { ODS } \\
\text { ODS }\end{array}$ & $\begin{array}{l}02 / 08 / 65 \\
02 / 08 / 65\end{array}$ & $\begin{array}{r}99,000 \\
100,000\end{array}$ & $\begin{array}{l}0.8877 \\
0.9023\end{array}$ & 209.07040 & $\begin{array}{l}34.20 \\
29.53\end{array}$ & $\begin{array}{l}499.53 \\
426.92\end{array}$ & $\begin{array}{l}11817 \\
11820\end{array}$ & $\begin{array}{l}9684 \\
9845\end{array}$ & $\begin{array}{l}0.079 ? \\
0.0800\end{array}$ & 412 & 403 & 2 \\
\hline
\end{tabular}




\begin{tabular}{|c|c|c|c|c|c|c|c|c|c|c|c|}
\hline IDENTIFICATION & DELAY & trans. & ENERGY & $x=5 E C$ & $\begin{array}{l}x= \\
R O\end{array}$ & $\underset{O P E N}{C O U}$ & & $\begin{array}{l}\text { FRAC, } \\
\text { Oo OC T }\end{array}$ & $\underset{\substack{\text { FAST } \\
\text { OPEN }}}{B}$ & AMPLE & $\begin{array}{l}\text { NO, }{ }_{\text {AVE }} \text { PTS } \\
\text { AVE }\end{array}$ \\
\hline 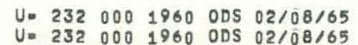 & $\begin{array}{l}101,000 \\
102,000\end{array}$ & $\begin{array}{l}0.9094 \\
0.8919\end{array}$ & $\begin{array}{l}204,95089 \\
20095194\end{array}$ & $\begin{array}{l}27.29 \\
32.88\end{array}$ & 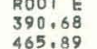 & $\begin{array}{l}\text { OEN } \\
11628 \\
11803\end{array}$ & $\begin{array}{l}\text { SAMPLE } \\
97,0 \\
917\end{array}$ & $\begin{array}{l}0.0808 \\
0.0816\end{array}$ & $\begin{array}{l}O P E N, \\
496 \\
496\end{array}$ & $\begin{array}{l}A M P L E \\
438 \\
486\end{array}$ & \\
\hline $\begin{array}{l}2320001960 \\
232 \\
23200 \\
1960\end{array}$ & $\begin{array}{l}103.000 \\
104.000\end{array}$ & $\begin{array}{l}0.0719 \\
0.8769 \\
0.7721\end{array}$ & $\begin{array}{l}2 \\
197,06890 \\
193.29734\end{array}$ & $\begin{array}{l}32.78 \\
37.72 \\
74.27\end{array}$ & 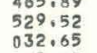 & $\begin{array}{l}11403 \\
11400 \\
11573\end{array}$ & $\begin{array}{l}911 \\
9292 \\
8_{248}\end{array}$ & $\begin{array}{l}0.0810 \\
0.0824 \\
0,0832\end{array}$ & $\begin{array}{l}486 \\
482 \\
513\end{array}$ & $\begin{array}{l}486 \\
473 \\
503\end{array}$ & \\
\hline 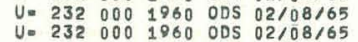 & $\begin{array}{l}105.000 \\
106,000\end{array}$ & $\begin{array}{l}0,7002 \\
0.7005\end{array}$ & $\begin{array}{l}189.63304 \\
186.071991\end{array}$ & $\begin{array}{l}102.37 \\
71.18\end{array}$ & $\begin{array}{l}90696 \\
40965 \\
970.92\end{array}$ & $\begin{array}{l}11588 \\
11439\end{array}$ & $\begin{array}{l}7489 \\
8240\end{array}$ & $\begin{array}{l}0.0840 \\
0,0848\end{array}$ & 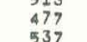 & 5 & \\
\hline 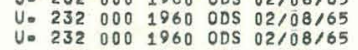 & $\begin{array}{l}107,000 \\
108,000\end{array}$ & $\begin{array}{l}0.8858 \\
0.9058\end{array}$ & 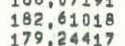 & $\begin{array}{l}71.26 \\
34.81 \\
3_{48}\end{array}$ & $\begin{array}{l}970.46 \\
470.46 \\
380.40\end{array}$ & $\begin{array}{l}11439 \\
11895 \\
11789\end{array}$ & $\begin{array}{l}8240 \\
9726 \\
9856\end{array}$ & $\begin{array}{l}0.0848 \\
0.0856\end{array}$ & $\begin{array}{l}537 \\
396\end{array}$ & $\begin{array}{l}526 \\
388 \\
385\end{array}-20$ & \\
\hline 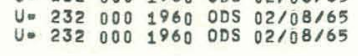 & $\begin{array}{l}109.000 \\
110,000\end{array}$ & $\begin{array}{l}0,9006 \\
0.9219\end{array}$ & $\begin{array}{l}175,97037 \\
172,98545\end{array}$ & $\begin{array}{l}230.06 \\
23.37\end{array}$ & $\begin{array}{l}398,79 \\
307,18\end{array}$ & $\begin{array}{l}11789 \\
11285 \\
11289\end{array}$ & $\begin{array}{l}9856 \\
9464 \\
9605\end{array}$ & $\begin{array}{l}0.0864 \\
0.0872 \\
0.0880\end{array}$ & $\begin{array}{l}332 \\
421\end{array}$ & 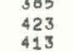 & \\
\hline 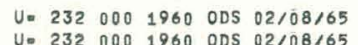 & 111.000 & 0.9110 & $\begin{array}{l}169,68623 \\
1.666096\end{array}$ & 26.78 & 348,80 & 11652 & 9796 & 0.0888 & 337 & 330 & \\
\hline 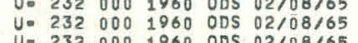 & $\begin{array}{l}\begin{array}{l}1122.000 \\
113\end{array} 000 \\
1.000\end{array}$ & $\begin{array}{ll}0 & 0,9248 \\
0,8331 & \end{array}$ & $\begin{array}{l}168,66968 \\
163,73279\end{array}$ & $\begin{array}{l}22.47 \\
35.70\end{array}$ & $\begin{array}{l}290,03 \\
456,80\end{array}$ & 11608 & $\begin{array}{l}9898 \\
9461\end{array}$ & & $\begin{array}{l}339 \\
356\end{array}$ & $\begin{array}{l}333 \\
348\end{array}$ & \\
\hline 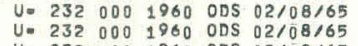 & $\begin{array}{l}\begin{array}{l}114,000 \\
115,000\end{array} \\
\end{array}$ & $\begin{array}{l}0: 8434 \\
0.7655\end{array}$ & $\begin{array}{l}160,87288 \\
158,08726\end{array}$ & $\begin{array}{l}48.92 \\
76.75\end{array}$ & $\begin{array}{l}620.50 \\
96504\end{array}$ & $\begin{array}{l}11512 \\
11186\end{array}$ & $\begin{array}{l}8960 \\
7903\end{array}$ & 0.0912 & $\begin{array}{l}304 \\
377 \\
377\end{array}$ & $\begin{array}{l}298 \\
298 \\
370\end{array}$ & \\
\hline 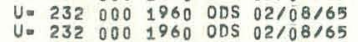 & $\begin{array}{l}116,000 \\
1117000\end{array}$ & $\begin{array}{ll}0.8265 \\
0 ; 8311\end{array}$ & $\begin{array}{l}155.37336 \\
152.7286\end{array}$ & $\begin{array}{l}54.74 \\
53.14\end{array}$ & $\begin{array}{l}682.27 \\
6560.69\end{array}$ & $\begin{array}{l}11275 \\
11418\end{array}$ & $\begin{array}{l}8600 \\
8650\end{array}$ & $\begin{array}{ll}0.0928 \\
0.0938\end{array}$ & 364 & 357 & \\
\hline 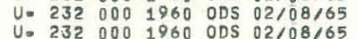 & $\begin{array}{l}118,000 \\
119000\end{array}$ & $\begin{array}{l}0.8515 \\
0.025\end{array}$ & $\begin{array}{l}150.15119 \\
167 \\
16316\end{array}$ & $\begin{array}{l}53.14 \\
4548 \\
548\end{array}$ & $\begin{array}{l}650.69 \\
561.65\end{array}$ & $\begin{array}{l}\begin{array}{l}11418 \\
11287\end{array}-V \\
11280\end{array}$ & $\begin{array}{l}8857 \\
8880\end{array}$ & $\begin{array}{l}0.0936 \\
0.0944\end{array}-0940$ & 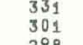 & $\begin{array}{l}324 \\
295\end{array}$ & \\
\hline $\begin{array}{lllll}32 & 000 & 1960 & 0 \mathrm{DS} & 02 / 08 / 65\end{array}$ & $\begin{array}{l}1290.000 \\
12000\end{array}$ & $\begin{array}{l}0: 8261 \\
0.793\end{array}$ & $\begin{array}{l}147: 038778 \\
145\end{array}$ & $\begin{array}{l}54.87 \\
94.63\end{array}$ & $\begin{array}{l}666.65 \\
140.24\end{array}$ & $\begin{array}{l}\begin{array}{l}11449 \\
10981\end{array}-2 \\
-\end{array}$ & $\begin{array}{l}8729 \\
7289\end{array}-\mathrm{l} \mathrm{l}$ & $\begin{array}{l}0.0952 \\
0.0960\end{array}$ & $\begin{array}{l}298 \\
316\end{array}$ & $\begin{array}{l}292 \\
310\end{array}$ & \\
\hline 2320001960 ODS $02 / 08 / 65$ & 121.000 & 0.6945 & $\begin{array}{l}142,79790 \\
1.40\end{array}$ & 104,72 & 251.33 & 10951 & $\begin{array}{l}7018 \\
8554\end{array}$ & 0.0968 & 308 & 302 & \\
\hline 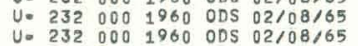 & $\begin{array}{l}123.000 \\
124.000\end{array}$ & $\begin{array}{l}0.8524 \\
0.9524\end{array}$ & $\begin{array}{l}148 \\
138,19198 \\
135,9790\end{array}$ & $\begin{array}{l}39 . \\
24 . \\
200\end{array}$ & $\begin{array}{l}460.121 \\
1.64 .80\end{array}$ & $\begin{array}{l}1102030 \\
10246\end{array}$ & $\begin{array}{l}8554 \\
805 \\
8090\end{array}$ & 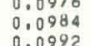 & $\begin{array}{l}350 \\
350 \\
329\end{array}$ & $\begin{array}{c}318 \\
343 \\
323\end{array}$ & \\
\hline 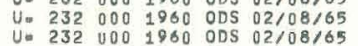 & $\begin{array}{l}125,000 \\
12500 \\
126,000\end{array}$ & $\begin{array}{lll}0.801 \\
0.0411 \\
0.734\end{array}$ & $\begin{array}{l}133,80506 \\
131.6859\end{array}$ & $\begin{array}{l}20.02 \\
49.69\end{array}$ & & $\begin{array}{l}10060 \\
10064\end{array}$ & $\begin{array}{l}8640 \\
7811\end{array}$ & $\begin{array}{l}0.0972 \\
0.1000\end{array}$ & $\begin{array}{l}359 \\
252 \\
295\end{array}$ & $\begin{array}{l}322 \\
247\end{array}$ & \\
\hline 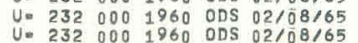 & $\begin{array}{l}127.000 \\
128000\end{array}$ & $\begin{array}{l}0.6219 \\
0.7633\end{array}$ & $\begin{array}{l}129,62391 \\
127,6045\end{array}$ & $\begin{array}{l}136.43 \\
137.59\end{array}$ & $\begin{array}{l}553128 \\
876,28\end{array}$ & $\begin{array}{l}10112 \\
10283\end{array}$ & $\begin{array}{l}8652 \\
5901\end{array}-12$ & $\begin{array}{l}0.1008 \\
0.1016\end{array}$ & $\begin{array}{l}295 \\
286 \\
286\end{array}$ & $\begin{array}{l}289 \\
280\end{array}$ & \\
\hline 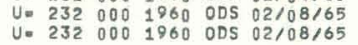 & $\begin{array}{l}129.000 \\
130.000\end{array}$ & 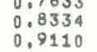 & $\begin{array}{l}125,63572 \\
123.71030\end{array}$ & $\begin{array}{l}52.35 \\
26,78\end{array}$ & $\begin{array}{l}\begin{array}{l}586,81 \\
297,89\end{array} \\
299\end{array}$ & $\begin{array}{l}10563 \\
10804 \\
10679\end{array}$ & $\begin{array}{l}74399 \\
8308\end{array}$ & $\begin{array}{l}0.1024 \\
0.1032\end{array}$ & $\begin{array}{l}248 \\
276 \\
276\end{array}$ & 2 & \\
\hline 0001960 ODS $02 / 08 / 65$ & 131.000 & 9365 & 1,82880 & 18,8 & 7,97 & & 9678 & & 225 & 206 & \\
\hline $\begin{array}{l}00 S 02 / 0865 \\
\text { ODS } 02 / 108 / 65\end{array}$ & $\begin{array}{l}132,000 \\
133,000\end{array}$ & $\begin{array}{ll}0,9366 \\
0,936\end{array}$ & $\begin{array}{l}119,98990 \\
118: 19232\end{array}$ & 年8 & $\begin{array}{l}227,70 \\
204.37\end{array}$ & 徒 & $\begin{array}{l}3520 \\
3520\end{array}$ & 0.1000 & 263 & 258 & \\
\hline $\begin{array}{l}2320000196000802 / 08 / 65 \\
232 \\
232\end{array}$ & $\begin{array}{l}134.000 \\
135\end{array}$ & 0.798 & $\begin{array}{l}116.43484 \\
11.4967\end{array}$ & 30.48 & & & & & 202 & 269 & \\
\hline 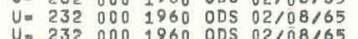 & $\begin{array}{l}136.000 \\
13600\end{array}$ & $\begin{array}{l}0.7982 \\
0.0095\end{array}$ & 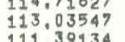 & $\begin{array}{l}64.79 \\
60.71\end{array}$ & $\begin{array}{l}693.37 \\
645.41\end{array}$ & $\begin{array}{l}11100 \\
11036\end{array}$ & $\begin{array}{l}8175 \\
8242\end{array}$ & $\begin{array}{l}0,1080 \\
0,11088\end{array}$ & $\begin{array}{l}266 \\
236 \\
236\end{array}$ & $\begin{array}{l}260 \\
231\end{array}$ & \\
\hline 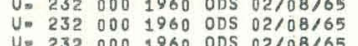 & $\begin{array}{l}1.37 .000 \\
138.000\end{array}$ & $\begin{array}{l}0.845 \\
0.6661\end{array}$ & $\begin{array}{l}1111,39134 \\
109,78282\end{array}$ & $\begin{array}{r}49.89 \\
116.71\end{array}$ & $\begin{array}{l}\begin{array}{r}526,56 \\
222.86\end{array} \\
2286\end{array}$ & $\begin{array}{l}10759 \\
10955\end{array}$ & $\begin{array}{l}8844 \\
6732\end{array}$ & $\begin{array}{l}0,1096 \\
0,1104\end{array}$ & $\begin{array}{l}296 \\
272\end{array}$ & $\begin{array}{l}290 \\
267\end{array}$ & \\
\hline $232 \quad 000 \quad 1960$ ODS $02 / 08 / 65$ & $\begin{array}{l}1399.000 \\
140.000\end{array}$ & $\begin{array}{l}0.5777 \\
0.7151\end{array}$ & $\begin{array}{l}108,20889 \\
106,60857\end{array}$ & $\begin{array}{c}160.08 \\
96.33 \\
96\end{array}-3$. & $\begin{array}{l}665: 19 \\
994,87\end{array}$ & $\begin{array}{l}11026 \\
10929\end{array}$ & $\begin{array}{l}5526 \\
7210\end{array}$ & $\begin{array}{l}0.1122 \\
0.1120\end{array}$ & $\begin{array}{l}268 \\
255\end{array}$ & $\begin{array}{l}263 \\
250\end{array}$ & \\
\hline $\begin{array}{lllll}232 & 000 & 1960 & 00 S & 02 / 08 / 65 \\
232 & 000 & 1960 & 00 S & 02 / 08 / 65\end{array}$ & $\begin{array}{l}141,000 \\
142,000\end{array}$ & $\begin{array}{l}0.8166 \\
0.854\end{array}$ & $\begin{array}{l}105.16099 \\
103.6898\end{array}$ & $\begin{array}{l}58,20 \\
45.25\end{array}-\mathrm{V}$ & 596,84 & 11045 & 8321 & 28 & 247 & 242 & \\
\hline 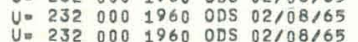 & $\begin{array}{l}143.000 \\
144000\end{array}$ & $\begin{array}{l}0.8769 \\
0.945\end{array}$ & $\begin{array}{l}102,23999 \\
100 \\
102859\end{array}$ & $\begin{array}{l}37,792 \\
37.47\end{array}$ & $\begin{array}{l}381.41 \\
3.551\end{array}$ & $\begin{array}{l}11192 \\
11192\end{array}$ & $\begin{array}{l}9055 \\
9075\end{array}$ & & 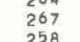 & 252 & \\
\hline 0001960 ODS 02 & 145,000 & ons & $\begin{array}{l}90.04395 \\
90.4895\end{array}$ & $=0$ & 20 & & & & 284 & 232 & \\
\hline 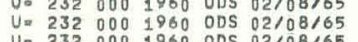 & $\begin{array}{l}146.000 \\
147.000\end{array}$ & $\begin{array}{l}0.9572 \\
0.9256\end{array}$ & 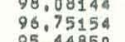 & $\begin{array}{l}12.55 \\
22.20\end{array}$ & $\begin{array}{l}12 \\
21\end{array}$ & & $\begin{array}{l}9618 \\
9444\end{array}$ & & $\begin{array}{l}269 \\
285\end{array}$ & $\begin{array}{l}264 \\
280 \\
280\end{array}$ & \\
\hline $\begin{array}{lll}232 \\
232\end{array}$ & $\begin{array}{l}148,000 \\
149000 \\
150,000\end{array}$ & $\begin{array}{l}0.9407 \\
0.9496 \\
0.9412\end{array}$ & $\begin{array}{l}95,44850 \\
94.17161 \\
92: 9218\end{array}$ & $\begin{array}{l}17.55 \\
14.84\end{array}$ & $\begin{array}{l}71,48 \\
44,00 \\
697,72\end{array}$ & & $\begin{array}{l}9564 \\
9531 \\
9513\end{array}$ & & $\begin{array}{l}274 \\
311 \\
312\end{array}$ & 268 & \\
\hline 5021 & .000 & 0.963 & 91.6935 & 10.80 & 03.39 & & 9497 & & 323 & & \\
\hline 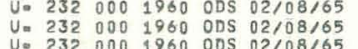 & $\begin{array}{l}152,000 \\
153,000\end{array}-1000$ & 0.9499 & $\begin{array}{l}90.49100 \\
89.3197\end{array}$ & $14,77,370$ & & & & & & & \\
\hline 0210 & $\begin{array}{l}154,000 \\
155000\end{array}$ & 0.9892 & 88,15584 & 8.97 & 25 & ${ }_{11119}^{1}$ & . & & 306 & 300 & \\
\hline $\begin{array}{l}1960 \\
1905 \\
19021 \\
1900\end{array}$ & 1556000 & $\because \theta$ & $\begin{array}{l}87.022202 \\
85.9093 \\
89.993\end{array}$ & $20^{\circ}$ & $101,1,5$ & $\begin{array}{l}113137 \\
10970\end{array}$ & , & & 127 & 100 & \\
\hline $\begin{array}{l}0220 \\
0220\end{array}$ & $\begin{array}{l}158.000 \\
158000\end{array}$ & $\begin{array}{l}0.948 \\
0.951\end{array}$ & $\begin{array}{r}84.81 \\
83.74\end{array}$ & $\begin{array}{l}1.5 \\
14\end{array}$ & & & & & $\begin{array}{l}330 \\
344\end{array}$ & 37 & \\
\hline 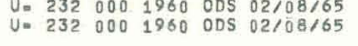 & $\begin{array}{l}159,000 \\
160.000\end{array}$ & $\begin{array}{l}0,9308 \\
0: 9372\end{array}$ & $\begin{array}{l}\frac{82,69683}{81 ; 66812} \\
86812\end{array}$ & $\begin{array}{l}20.58 \\
18.61\end{array}$ & $\begin{array}{l}187: 20 \\
168: 21\end{array}$ & 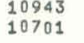 & $\begin{array}{l}9396 \\
9251\end{array}$ & 82 & $\begin{array}{l}353 \\
326\end{array}$ & $\begin{array}{l}346 \\
319\end{array}$ & \\
\hline 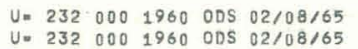 & $\begin{array}{l}161.000 \\
162.000\end{array}$ & $\begin{array}{l}0.9650 \\
0.8992\end{array}$ & $\begin{array}{l}80,6567 \\
79.6640\end{array}$ & $\begin{array}{l}10.22 \\
30.51\end{array}$ & 1.8 & 10605 & 9440 & 1288 & 401 & 3 & \\
\hline $\begin{array}{l}19600050210 \\
1960 \\
1905 \\
02210\end{array}$ & 163.000 & 0.895 & 78.68960 & 31. & & & & & 398 & & \\
\hline 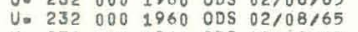 & 25 & 0.000 & 76.79354 & 41. 4, & & & $\begin{array}{l}84 \\
70\end{array}-2-10$ & & $\begin{array}{l}415 \\
440\end{array}$ & 87 & \\
\hline 021 & 166.00 & 0.449 & $\begin{array}{l}75,87110 \\
77.95518\end{array}$ & 209 & & & 43 & & No $-2+2$ & 401 & \\
\hline 021 & $\begin{array}{l}167.000 \\
168.000\end{array}$ & $\begin{array}{l}0.2666 \\
0.4654\end{array}$ & $\begin{array}{l}74.96518 \\
74.07540\end{array}$ & $\begin{array}{l}379.71 \\
219.65\end{array}$ & 990 & 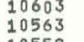 & $\begin{array}{l}26 \\
45 \\
45\end{array} \mathrm{~J} \mathrm{l}$ & $\begin{array}{l}0.1 \\
0.1\end{array}$ & $\begin{array}{l}488 \\
448\end{array}$ & 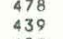 & \\
\hline 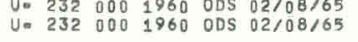 & $\begin{array}{l}169.000 \\
170,000\end{array}$ & $\begin{array}{l}0,7399 \\
0,8891\end{array}$ & $\begin{array}{l}73,20136 \\
72,34270\end{array}$ & $\begin{array}{l}84.97 \\
33.77\end{array}-1$ & $\begin{array}{l}727.02 \\
287.23\end{array}$ & $\begin{array}{l}10552 \\
10549\end{array}$ & $\begin{array}{l}7240 \\
8650\end{array}-100$ & $\begin{array}{l}0,1522 \\
0,1300\end{array}$ & $\begin{array}{l}493 \\
444\end{array}$ & 年353 & \\
\hline 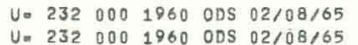 & $\begin{array}{l}171.000 \\
172\end{array}$ & 0.9283 & $\begin{array}{r}71,4990 \\
7006700\end{array}-30$ & 21. & $\begin{array}{l}180,83 \\
192.2\end{array}$ & 1073! & 919 & 68 & 415 & 407 & \\
\hline 0210 & $\begin{array}{l}173,000 \\
174000\end{array}$ & $0 \% 9$ & $\begin{array}{l}698,855 \\
609054\end{array}$ & , & & & 90 & & No & & \\
\hline $\begin{array}{lllll}232 & 000 & 1960 & 005 & 02 \\
232 & 000 & 1960 & 005 & 02\end{array}$ & 175 & 80 & 68.26 & & & & & & 519 & & \\
\hline $\begin{array}{ll}1960 & 0 D S \\
192 / 0\end{array}$ & 177.0 & 0.9265 & 66.73 & & & & & & 562 & 551 & \\
\hline 0001960 ODS $02 / 08 / 65$ & 17 & 0. & 年 & & & & ${ }^{9} 125$ & & $2<4$ & 513 & \\
\hline 0001960 ODS $02 / 0$ & 181.0 & 9358 & 3,8 & 13.16 & & & & & 488 & $78.25>3$ & \\
\hline 0220 & 2000 & ore & $\because$ & & & & & & 400 & & \\
\hline 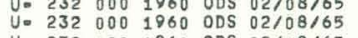 & $\begin{array}{l}183.000 \\
184.000\end{array}$ & $\begin{array}{l}0: 9531 \\
0: 9413\end{array}$ & $\begin{array}{l}62.42 \\
61.75\end{array}$ & 13. & & & $\begin{array}{l}91 \\
88\end{array}$ & & 500 & $\begin{array}{l}490 \\
454\end{array}$ & \\
\hline $\begin{array}{lll}23 \\
23\end{array}$ & $\begin{array}{l}185.000 \\
186.000\end{array}$ & $\begin{array}{l}0.92 \\
0.88\end{array}$ & $\begin{array}{l}61.08 \\
60.43\end{array}$ & $\begin{array}{l}21.3 \\
35.0\end{array}$ & & & $\begin{array}{l}87 \\
84\end{array}$ & & $\begin{array}{l}503 \\
382\end{array}$ & & \\
\hline 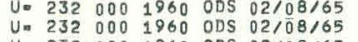 & $\begin{array}{l}187.000 \\
188.000\end{array}$ & $\begin{array}{l}0: 839 \\
0,895\end{array}$ & $\begin{array}{l}59,78 \\
59.15\end{array}-15$ & 31 & & & & & $\begin{array}{l}37 \\
40\end{array}$ & 400 & \\
\hline 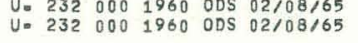 & $\begin{array}{l}189,000 \\
190: 000\end{array}$ & $\begin{array}{l}0.952 \\
0.935\end{array}$ & $\begin{array}{l}58,5287 \\
57.9142\end{array}$ & 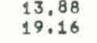 & 145,79 & $\begin{array}{l}1045 \\
10299\end{array}$ & $\begin{array}{l}8913 \\
8883\end{array}$ & $\begin{array}{l}1512 \\
1520\end{array}$ & 40 & 399 & \\
\hline $\begin{array}{l}1960 \text { ODS } 02 / 08 / 65 \\
1960 \text { ODS } 02 / 08 / 65\end{array}$ & 191.000 & 0.9332 & 57,30 & 19.8 & & 0216 & 8791 & & 37 & 369 & \\
\hline $\begin{array}{l}021 \\
021\end{array}$ & 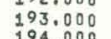 & & $\begin{array}{l}30.12 \\
5.55\end{array}$ & 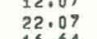 & & & & & & & \\
\hline 021 & & & 40 & & & & & & 36 & & \\
\hline $\begin{array}{l}02 / 08765 \\
02 / 08 / 65\end{array}$ & & & (1) 1009 & & & & & & & & \\
\hline 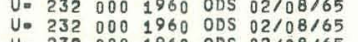 & $\begin{array}{l}198.000 \\
999.000\end{array}$ & $\begin{array}{l}7907 \\
6729\end{array}$ & $\begin{array}{l}3.32884 \\
2.79422\end{array}$ & $\begin{array}{l}67.45 \\
3.76\end{array}$ & & & 6124 & & & 329 & \\
\hline & 200.000 & & & & & & & & & & \\
\hline
\end{tabular}




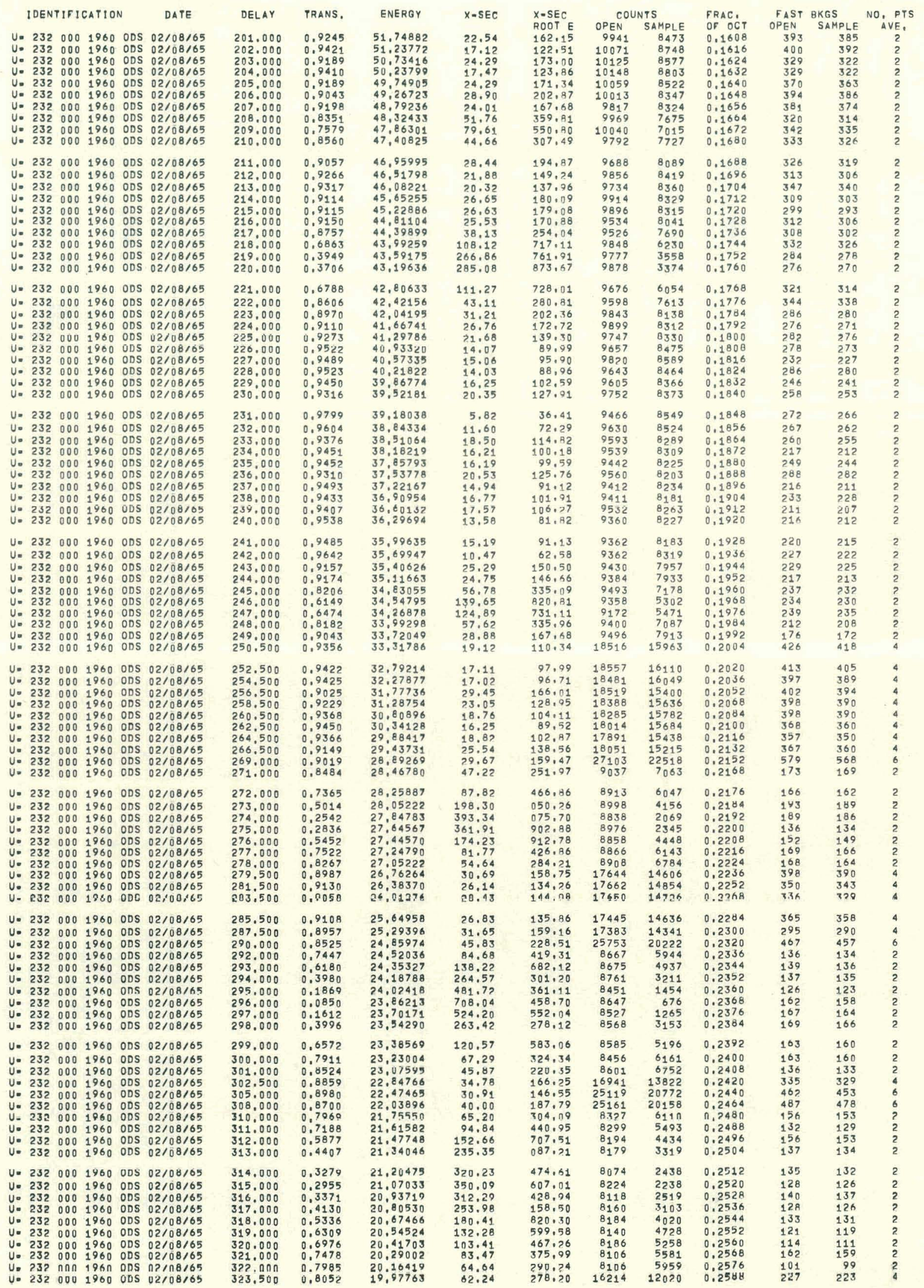




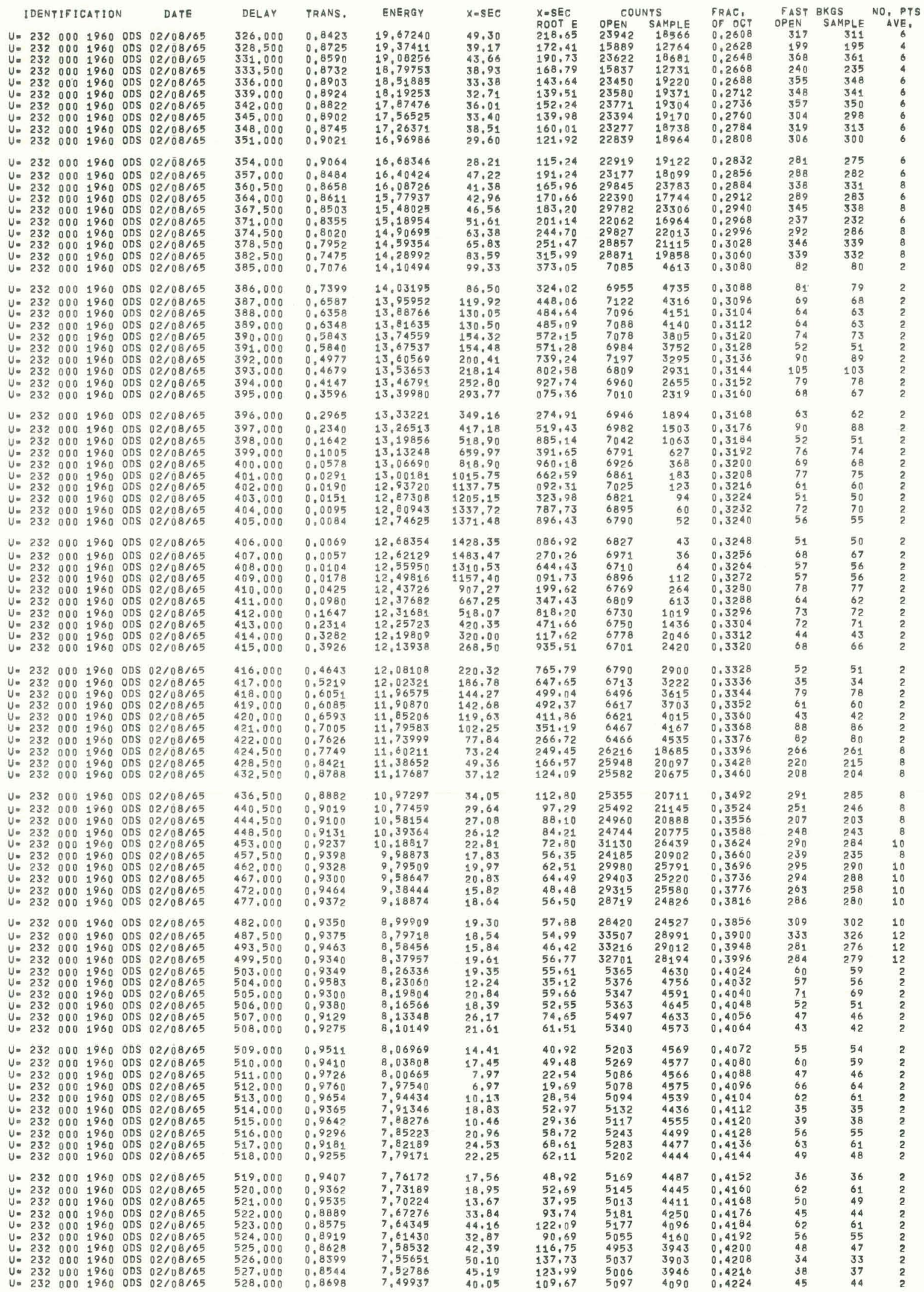




\begin{tabular}{|c|c|c|c|c|c|c|c|c|c|c|c|c|c|c|c|}
\hline \multicolumn{4}{|c|}{ IDENTIFICATION } & DATE & DELAY & TRANS. & ENERGY & $x-$ SEC & $\begin{array}{l}X \text {-SEC } \\
\text { ROOT E }\end{array}$ & $\begin{array}{c}\text { COU } \\
\text { OPEN }\end{array}$ & $\begin{array}{l}\text { ITS } \\
\text { SAMPLE }\end{array}$ & $\begin{array}{l}\text { FRAC, } \\
\text { OF OCT }\end{array}$ & $\begin{array}{l}F A S T \\
\text { OPEN }\end{array}$ & $\begin{array}{l}\text { BKGS } \\
\text { SAMPLE }\end{array}$ & $\begin{array}{c}\text { NO, PTS } \\
\text { AVE, }\end{array}$ \\
\hline$U=232$ & 000 & 1960 & ODS & $02 / 08 / 65$ & 529.000 & 0,8786 & 7,47104 & 37.18 & 101.61 & 4974 & 4032 & 0.4232 & 57 & 56 & 2 \\
\hline U. 232 & 000 & 1960 & ODS & $02 / 08 / 65$ & 530.000 & 0.9013 & 7,44288 & 29.85 & 81.44 & 5107 & 4246 & 0.4240 & 61 & 60 & 2 \\
\hline$U=232$ & 000 & 1960 & ODS & $02 / 08 / 65$ & 531.000 & 0.8943 & 7,41487 & 32.08 & 87.35 & 5028 & 4148 & 0.4248 & 76 & 74 & 2 \\
\hline$U=232$ & 000 & 1960 & ODS & $02 / 08 / 65$ & 532.000 & 0.8759 & $7,3870 ?$ & 38.05 & 103.42 & 5129 & 4145 & 0.4256 & 61 & 60 & 2 \\
\hline$U=232$ & 000 & 1960 & ODS & $02 / 08 / 65$ & 533.000 & 0.9100 & 7,35933 & 27.08 & 73.47 & 5008 & 4204 & 0.4264 & 30 & 29 & 2 \\
\hline$U=232$ & 000 & 1960 & ODS & $02 / 08 / 65$ & 534.000 & 0,9302 & 7,33179 & 20.78 & 56.27 & 4965 & 4261 & 0.4272 & 64 & 62 & 2 \\
\hline$U=232$ & 000 & 1960 & ODS & $02 / 08 / 65$ & 535.000 & 0,9175 & 7,30441 & 24.74 & 66.85 & 4925 & 4169 & 0.4280 & $5_{1}$ & 50 & 2 \\
\hline$U=232$ & 000 & 1960 & ODS & $02 / 08 / 65$ & 536.000 & 0,9538 & 7.27718 & 13.59 & 36.66 & 4925 & 4333 & $0.42 B 8$ & 43 & 42 & 2 \\
\hline $\begin{array}{l}U: 232 \\
Y: 230\end{array}$ & 000 & 1960 & ODS & $02 / 08 / 65$ & 537.000 & 0.9019 & 7.25010 & 29.68 & 79.87 & 4920 & 4092 & 0.4296 & 57 & 56 & 2 \\
\hline & & 1960 & ODS & & 538.000 & 0.9232 & 7,22317 & 22.95 & 61.69 & 4999 & ${ }^{4} 254$ & 0.4304 & 33 & 32 & 2 \\
\hline$U=232$ & 000 & 1960 & ODS & $02 / 08 / 65$ & 539,000 & 0,9459 & 7,19640 & 15,97 & 42,83 & 4889 & 4262 & 0.4312 & 44 & 43 & 2 \\
\hline U. 232 & 000 & 1960 & ODS & $02 / 08 / 65$ & 540.000 & 0.9023 & 7,16977 & 29.54 & 79.09 & 4911 & 4082 & 0.4320 & 47 & 47 & 2 \\
\hline$U=232$ & 000 & 1960 & ODS & $02 / 08 / 65$ & 541.000 & 0.9357 & 7.14329 & 19.08 & 50.99 & 4776 & 4115 & 0.4328 & 46 & 45 & 2 \\
\hline$U=232$ & 000 & 1960 & ODS & $02 / 08 / 65$ & 542.000 & 0.9006 & 7,11695 & 30.06 & 80.18 & 4933 & 4089 & 0.4336 & 40 & 39 & 2 \\
\hline$U=232$ & 000 & 1960 & ODS & $02 / 08 / 65$ & 544.000 & 0.9228 & 7.06472 & 23.08 & 61.36 & 14357 & 12177 & 0.4352 & 149 & 146 & 6 \\
\hline$U=232$ & & 1960 & ODS & $02108 / 65$ & 547.500 & $0.915 ?$ & 6,97468 & 25.45 & 67.22 & 18940 & 15883 & 0,4380 & 173 & 169 & 8 \\
\hline$U=232$ & 000 & 1960 & ODS & $02108 / 65$ & 551.500 & 0.9060 & 6,87387 & 28.34 & 74.31 & 18685 & 15422 & 0,4412 & 187 & 184 & 8 \\
\hline U. 232 & 000 & 1960 & ODS & $02 / 08 / 65$ & 555.500 & 0.8886 & 6.77524 & 33,92 & 88,29 & 18535 & 14936 & 0.4444 & 196 & 192 & 8 \\
\hline$U=232$ & 000 & 1960 & ODS & $02 / 08 / 65$ & 560.000 & 0.8524 & 6.66679 & 45.85 & 118,39 & 23332 & 18127 & 0,4480 & 228 & 224 & 10 \\
\hline U. 232 & 000 & 1960 & ODS & $02 / 08 / 65$ & 564,500 & 0.8410 & 6,56092 & 49.73 & 127,38 & 18223 & 14023 & 0.4516 & 227 & 223 & 8 \\
\hline$U=232$ & 000 & 1960 & ODS & $02 / 08 / 65$ & 568.500 & 0.7997 & 6.46892 & 64.20 & 163,30 & 18108 & 13283 & 0.4548 & 202 & 198 & 8 \\
\hline$U=232$ & 000 & 1960 & ODS & $02 / 08 / 65$ & 571.000 & 0.7917 & 6,41240 & 87.08 & 169.86 & 4394 & 3195 & 0.4568 & 39 & 38 & 2 \\
\hline U. 232 & 000 & 1960 & 005 & $02 / 08 / 65$ & 572.000 & 0.7258 & 6,38990 & 92.06 & 232.71 & 4490 & 2994 & 0.4576 & $s_{1}$ & 50 & 2 \\
\hline U. 232 & 0.00 & 1960 & ODS & $02 / 08 / 65$ & $57 \overline{3} .000$ & 0.6988 & 6.36771 & 102.93 & 259,73 & 4594 & 2951 & 0.4584 & 61 & 60 & 2 \\
\hline U. 232 & 000 & 1960 & ODS & $02 / 08 / 65$ & 574.000 & 0.6762 & 6.34554 & 112.38 & 283.08 & 4480 & 2786 & 0.4592 & 49 & 48 & 2 \\
\hline U. 232 & 000 & 1960 & ODS & $02 / 08 / 65$ & 575.000 & 0.6703 & 6,32349 & 114.90 & 288,94 & 4461 & 2750 & 0.4600 & 57 & 56 & 2 \\
\hline$U=232$ & 000 & 1960 & ODS & $02 / 08 / 65$ & 576.000 & 0.6367 & 6,30155 & 129.65 & 325,46 & 4389 & 2571 & 0.4608 & 34 & 33 & 2 \\
\hline$U=232$ & 000 & 1960 & ODS & $02 / 08 / 65$ & 577.000 & 0.6064 & 6,27973 & 143.64 & 359,95 & 4380 & 2445 & 0.4616 & 34 & 33 & 2 \\
\hline$U=232$ & 000 & 1960 & ODS & $02 / 08 / 65$ & 578.000 & 0.5622 & 6,25802 & 165.42 & 413,82 & 4528 & 2344 & 0,4624 & 44 & 43 & 2 \\
\hline$U=232$ & 000 & 1960 & ODS & $02 / 08 / 65$ & 579.000 & 0,5058 & 6,23642 & 195.75 & 488,85 & 4375 & 2038 & 0,4632 & 37 & 36 & 2 \\
\hline$U=232$ & 000 & 1960 & ODS & $02 / 08 / 65$ & 580.000 & 0.4648 & 6,21493 & 220.06 & 548,61 & 4300 & 1841 & 0,4640 & 69 & 67 & 2 \\
\hline$U=232$ & 000 & 1960 & ODS & $02 / 08 / 65$ & 581.000 & 0.3536 & 6.19356 & 298,60 & 743.11 & 4362 & 1421 & 0.4648 & 38 & 37 & 2 \\
\hline$U=232$ & 000 & 1960 & ODS & $02 / 08 / 65$ & 582.000 & 0.2921 & 6.17229 & 353.49 & 878,21 & 4277 & 1150 & 0.4656 & 58 & 56 & 2 \\
\hline U. 232 & 000 & 1960 & ODS & $02 / 08 / 65$ & 583.000 & 0.2114 & 6.15114 & 446.25 & 106.77 & 4433 & 863 & 0.4664 & 43 & 42. & 2 \\
\hline U. 232 & 000 & 1960 & ODS & $02 / 08 / 65$ & 584.000 & 0.1268 & 6.13009 & 593.16 & 468,60 & 4282 & 500 & 0.4672 & 46 & 45 & 2 \\
\hline$U=232$ & 000 & 1960 & ODS & $02 / 08 / 65$ & 585.000 & 0.0837 & 6.10915 & 712.37 & 760.73 & 4235 & 326 & 0,4680 & 53 & 51 & 2 \\
\hline U. 232 & 000 & 1960 & ODS & $02 / 08 / 65$ & 586.000 & 0.0463 & 6,08832 & 882.24 & 176,89 & 4202 & 179 & 0,4688 & 59 & 57 & 2 \\
\hline$U=232$ & 000 & 1960 & ODS & $02 / 08 / 65$ & 587.000 & 0.0286 & 6.06759 & 1021.28 & 515.66 & 4270 & 112 & 0,4696 & 76 & 74 & 2 \\
\hline$U=232$ & 000 & 1960 & ODS & $02 / 08 / 65$ & 588.000 & 0.0197 & 6.04697 & 1128.23 & 774.40 & 4238 & 70 & 0.4704 & 67 & 66 & 2 \\
\hline$U=232$ & 0.00 & 1960 & ODS & $02 / 08 / 65$ & 589.000 & 0.0208 & 6.02646 & 1114,58 & 736.15 & 4339 & 82 & 0.4712 & 39 & 38 & 2 \\
\hline U. 232 & 000 & 1960 & ODS & $02 / 08 / 65$ & 590,000 & 0.0074 & 6,00604 & 1409.27 & 453.72 & 4269 & 29 & 0.4720 & 59 & 57 & 2 \\
\hline$U=232$ & 000 & 1960 & ODS & $02 / 08 / 65$ & 591.000 & 0.0116 & 5,98574 & 1280.95 & 133,95 & 4235 & 45 & 0.4728 & 35 & 34 & 2 \\
\hline$y=232$ & 000 & 1960 & ODS & $02 / 08 / 65$ & 592.000 & 0.0113 & 5,96553 & 1287,46 & 144,55 & 4105 & 42 & 0,4736 & 60 & 59 & 2 \\
\hline U. 232 & 000 & 1960 & ODS & $02 / 08 / 65$ & 593.000 & 0.0069 & 5,94543 & 1428.74 & 483,74 & 4110 & 26 & 0.4744 & 75 & 73 & 2 \\
\hline U. 232 & 000 & 1900 & ODS & $02 / 08 / 65$ & 594.000 & 0.0130 & 5,92543 & 1247.13 & 035,79 & 4277 & 51 & 0.4752 & 45 & 44 & 2 \\
\hline U. 232 & 000 & 1960 & ODS & $02 / 08 / 65$ & 595.000 & 0.0041 & 5,90553 & 1581.00 & 842.03 & 4192 & 15 & 0.4760 & 54 & 53 & 2 \\
\hline$U=232$ & 000 & 1960 & ODS & $02 / 08 / 65$ & 596.000 & 0.0158 & 5.88573 & 1191.54 & 890.75 & 4113 & 59 & 0.4768 & 61 & 60 & 2 \\
\hline U. 232 & 000 & 1960 & ODS & $02 / 08 / 65$ & 597.000 & 0.0378 & 5,86002 & 940.57 & 278.05 & 4109 & 143 & 0,4776 & 48 & 47 & 2 \\
\hline U. 232 & 000 & 1960 & ODS & $02 / 08 / 65$ & 598.000 & 0,0860 & 5,84642 & 704.63 & 703,75 & 4171 & 330 & 0,4784 & 66 & 65 & 2 \\
\hline$U=232$ & 000 & 1960 & ODS & $02 / 08 / 65$ & 599.000 & 0.1479 & 5.82692 & 548.83 & 324.83 & 4101 & 558 & 0.4792 & 75 & 73 & 2 \\
\hline$U=232$ & 000 & 1960 & ODS & $02 / 08 / 65$ & 600.000 & 0.2329 & 5,80751 & 418,45 & 008,42 & 4138 & 887 & 0,4800 & 30 & 30 & 2 \\
\hline$U=232$ & 000 & 1960 & ODS & $02 / 08 / 65$ & 601.000 & 0,2932 & 5,78820 & 352.36 & 847,74 & 4013 & 1084 & 0,4808 & 78 & 76 & 2 \\
\hline$U=232$ & 000 & 1960 & ODS & $02 / 08 / 65$ & 602.000 & 0,3851 & 5,76890 & 274.05 & 658,24 & 4075 & 1445 & 0,4816 & 57 & 58 & 2 \\
\hline$U=232$ & 000 & 1960 & ODS & $02 / 08 / 65$ & 603.000 & 0.4494 & 5,74987 & 229.74 & 550.89 & 3909 & 1618 & 0,4824 & 62 & 61 & 2 \\
\hline$U=232$ & 000 & 1960 & ODS & $02 / 08 / 65$ & 604.000 & 0.5097 & 5,73085 & 193,56 & 463,36 & 3941 & 1851 & 0.4832 & 65 & 63 & 2 \\
\hline$U=232$ & 000 & 1960 & ODS & $02 / 08 / 65$ & 605.000 & 0.5710 & 5.71192 & 160.93 & 384.62 & 3925 & 2065 & 0.484 & 54 & 53 & 2 \\
\hline$U=232$ & 000 & 1960 & ODS & $02 / 08 / 65$ & 606.000 & 0.5985 & 5.69308 & 147.45 & 351.81 & 4081 & 2251 & 0.4848 & 59 & 57 & 2 \\
\hline$U=232$ & 000 & 1960 & ODS & $02 / 08 / 65$ & 607.000 & 0.6108 & 5.67434 & 141.59 & 337.27 & 4072 & 2292 & 0.4856 & 51 & 50 & 2 \\
\hline U. 232 & 000 & 1960 & ODS & $02 / 08 / 65$ & 608.000 & 0.6590 & 5.65569 & 119.76 & 284,82 & 3969 & 2411 & 0,4864 & 45 & 44 & 2 \\
\hline$U=232$ & 000 & 1960 & ODS & $02 / 08 / 65$ & 609.000 & 0,7184 & 5,63713 & 94.97 & 225,48 & 3915 & 2593 & 0,4872 & 38 & 37 & 2 \\
\hline $4=232$ & 000 & 1960 & ons & $02 / 08 / 65$ & 610.000 & 0.6980 & 5,61866 & 103.26 & 244,76 & 3952 & 2543 & 0.4880 & 70 & 68 & 2 \\
\hline$y=232$ & 000 & 1960 & ODS & $02 / 08 / 65$ & 613.000 & 0.7669 & 5,56380 & 76.24 & 179,82 & 19733 & 13956 & 0.4904 & 257 & 252 & 10 \\
\hline$U=232$ & 000 & 1960 & ODS & $02 / 08 / 65$ & 618.500 & 0.8435 & $5.46=$ & 48. & $114 \cdot 26$ & 23312 & $18_{1}$ & 0.45 & 321 & 305 & 12 \\
\hline$U=232$ & 000 & 1960 & ODS & $02 / 08 / 65$ & 624.500 & 0.8 & 5,360 & 45. & 106. & 22887 & 179 & 0.49 & 335 & 329 & 12 \\
\hline$U=232$ & 000 & 1960 & ODS & $02 / 08 / 65$ & 630.500 & 0.8773 & 5,25923 & 37.60 & 86.22 & 22481 & $18_{182}$ & 0,5044 & 337 & 330 & 12 \\
\hline U. 232 & 000 & 1960 & ODS & 02108165 & 636,500 & 0.8875 & 5,26055 & 34.26 & 77.84 & 22021 & 18016 & 0,5092 & 321 & 314 & 12 \\
\hline$U=232$ & 000 & 1960 & ODS & $02 / 08 / 65$ & 646.000 & 0.9120 & 5,00988 & 26.45 & $59 \cdot 21$ & 46466 & 39054 & 0,5168 & 711 & 697 & 26 \\
\hline$U=232$ & 000 & 1960 & ODS & 02108 & 659 & 0. & $4, e_{1}$ & 19. & 42.84 & 44600 & 383 & & 810 & 794 & 26 \\
\hline$U=232$ & 000 & 1960 & ODS & $02 / 08 / 65$ & 673.000 & 0.9393 & 4,61596 & 17.99 & 38.64 & 48925 & 42337 & 0,5384 & 752 & 737 & 30 \\
\hline$U=$ & 000 & 1960 & ODS & $02 / 08 / 65$ & 688,500 & 0,9316 & 4,41047 & 20.35 & 42.74 & 49816 & 42779 & 0,5508 & 919 & 901 & 32 \\
\hline U. 232 & & & ODS & $02 / 08 / 65$ & 704,500 & 0.9334 & 4,21241 & 19.80 & 40.64 & 46859 & 40337 & 0,5636 & 869 & 852 & 32 \\
\hline$y=232$ & 000 & 1960 & ODS & $02 / 08 / 65$ & 721.500 & 0.9 & 4.01624 & 18.58 & 37.24 & 49595 & 42884 & 0.5 & 1158 & 1134 & 36 \\
\hline$y=232$ & 000 & 1960 & ODS & $02108 / 65$ & 740,500 & & 3.81279 & 18, & 36.20 & 51766 & 44704 & 0,5924 & 1195 & 1171 & 40 \\
\hline$U=232$ & 000 & 1960 & ODS & $02 / 008 / 65$ & 761.000 & 0.9568 & 3,61013 & 12.68 & 24.09 & 50189 & 44105 & 0.6088 & 1353 & 1326 & 42 \\
\hline$U=232$ & 000 & 1960 & ODS & $02 / 08 / 65$ & 783.000 & 0.9469 & 3.41012 & 15.68 & 28.95 & 50890 & 44337 & 0.6264 & 1406 & 1378 & 46 \\
\hline U. 232 & 000 & 1960 & ODS & $02 / 08 / 65$ & 807.000 & 0.9418 & 3,21030 & 17.22 & 30,85 & 49883 & 43308 & 0.6456 & 1601 & 1569 & 50 \\
\hline$U=232$ & 000 & 1960 & ODS & $02 / 08 / 65$ & 833,000 & 0,9399 & 3,01302 & 17.81 & 30.91 & 46513 & 40322 & 0.6664 & 1764 & 1728 & 54 \\
\hline$U=232$ & 000 & 1960 & ODS & $02 / 08 / 65$ & 862.000 & 0.9322 & 2.81370 & 20.16 & 33.81 & 42656 & 36683 & 0.68 & 2274 & 2229 & 62 \\
\hline$U=232$ & 000 & 1960 & ODS & $02 / 08 / 65$ & 894.500 & 0.9372 & 2.61295 & 18.62 & 30. & 34804 & 30084 & 0.7156 & 2776 & 2720 & 68 \\
\hline$U=232$ & 000 & 1960 & ODS & $02 / 08 / 65$ & 931.000 & 0.9298 & $2.4120^{\circ}$ & 20.91 & 32.48 & 26064 & 22240 & 0.7448 & 4360 & 4273 & 78 \\
\hline
\end{tabular}




\begin{tabular}{|c|c|c|c|c|c|c|c|c|c|c|c|c|c|c|c|}
\hline IDENT & TIFIC & CATION & & DATE & DELAY & TRANS, & ENERGY & $x-\mathrm{SEC}$ & $\begin{array}{l}X=S E C \\
R O O T E\end{array}$ & ${ }_{O P E N}^{C O L}$ & MPLE & $\begin{array}{l}\text { FRAC, } \\
\text { OF OCT }\end{array}$ & $\begin{array}{l}\text { FAST } \\
\text { OPEN }\end{array}$ & $\begin{array}{l}\text { SKGS } \\
\text { SAMPLEE }\end{array}$ & $\begin{array}{l}\text { No, PTS } \\
\text { AVE, }\end{array}$ \\
\hline & 000 & & DS & $02 / 08 / 65$ & 14.500 & 0.9809 & 9943.89526 & 11.70 & $\begin{array}{r}\text { R00T E } \\
1160,88\end{array}$ & $\begin{array}{l}\text { OPEN } \\
35704\end{array}$ & $\begin{array}{l}\text { AMPLE } \\
3246 E\end{array}$ & of. OCT & $\begin{array}{l}\text { OPEN } \\
14899\end{array}$ & $\begin{array}{l}\text { SAMPLEE } \\
14750\end{array}$ & \\
\hline U. 232 & 000 & $\begin{array}{l}1961 \\
1961\end{array}$ & ODS & 02108165 & 15.500 & 0.9786 & 8702.20178 & $\begin{array}{l}11,100 \\
12.60\end{array}$ & $\begin{array}{l}1100.00 \\
1175.56\end{array}$ & $\begin{array}{l}33220 \\
33220\end{array}$ & $3015^{9}$ & 0.0129 & $\begin{array}{l}12786 \\
12278\end{array}$ & 12658 & $\begin{array}{l}2 \\
2 \\
\end{array}$ \\
\hline$y=232$ & 000 & 1961 & ODS & $02 / 08 / 65$ & 16.500 & 0.9752 & 7679.35352 & 14.58 & 1277,74 & 36301 & 32846 & 0.0137 & 8773 & 8686 & 2 \\
\hline $\begin{array}{l}U=232 \\
y=230\end{array}$ & 000 & 1961 & ODS & $\begin{array}{l}02 / 08 / 65 \\
0 / 28 / 65\end{array}$ & $\begin{array}{l}17.500 \\
18.500\end{array}$ & 0.9553 & 6826.78851 & 26.61 & 2198,77 & 34271 & 30368 & 0.0146 & 8410 & 8326 & 2 \\
\hline $\begin{array}{l}u=232 \\
u=232 \\
=232\end{array}$ & $\begin{array}{l}000 \\
000\end{array}$ & $\begin{array}{l}1961 . \\
1961\end{array}$ & $\begin{array}{l}\text { ODS } \\
\text { ODS }\end{array}$ & $\begin{array}{l}02 / 08 / 15 \\
02 / 108 / 65\end{array}$ & $\begin{array}{l}18.500 \\
19.500\end{array}$ & $\begin{array}{l}0,9623 \\
0,9626\end{array}$ & $\begin{array}{l}6108.10416 \\
5498.23535\end{array}$ & $\begin{array}{l}22.34 \\
22,14\end{array}$ & $\begin{array}{l}\begin{array}{l}7745,93 \\
1642,05\end{array} \\
164\end{array}$ & $\begin{array}{l}38060 \\
37147\end{array}$ & $\begin{array}{l}30406 \\
33167\end{array}$ & $\begin{array}{l}0.01174 \\
0,0162\end{array}$ & $\begin{array}{l}6140 \\
6937\end{array}$ & $\begin{array}{l}8.059 \\
6868\end{array}$ & $\begin{array}{l}? \\
2\end{array}$ \\
\hline 32 & 000 & 1961 & ODS & 3,05 & & 9686 & 497490540 & & $\begin{array}{l}130^{7} \\
\end{array}$ & 38063 & 34734 & 0,0171 & 4779 & 4731 & $\frac{2}{2}$ \\
\hline 32 & 000 ? & 1961 & ODS & $02 / 08 / 65$ & $21: 5 \mathrm{c}$ & 0,9682 & 4522.88586 & ${ }_{18} 8: 79$ & $1263: 54$ & 35547 & 31920 & 0.0179 & 5543 & 5488 & $i$ \\
\hline 32 & 000 & & ODS & $02 / 08 / 65$ & 22.500 & 0,9619 & 4129.78564 & 22.57 & 1450,56 & 36242 & 32333 & 0.0187 & 4453 & 4408 & 2 \\
\hline U. 232 & 000 & 1961 & ODS & $02 / 08 / 65$ & 23.500 & 0,9757 & 3785.79266 & 14.32 & 881,18 & 37534 & 33963 & 0.0196 & 3482 & 3447 & 2 \\
\hline 32 & 000 & 1961 & ODS & $\begin{array}{l}02 / 08 / 65 \\
02 / 08 / 65\end{array}$ & $\begin{array}{l}24,500 \\
25.500\end{array}$ & $\begin{array}{l}0.9661 \\
0.9692\end{array}$ & $\begin{array}{l}3483.05539 \\
3215.23105\end{array}$ & $\begin{array}{l}20.05 \\
18.21\end{array}$ & $\begin{array}{l}1183,54 \\
1032,34\end{array}$ & $\begin{array}{l}37826 \\
37135\end{array}$ & $\begin{array}{l}33892 \\
33738\end{array}$ & $\begin{array}{l}0,0204 \\
0,0212\end{array}$ & $\begin{array}{l}4122 \\
3822\end{array}$ & $\begin{array}{l}4080 \\
3784\end{array}$ & $\frac{2}{2}$ \\
\hline & $\begin{array}{l}000 \\
000\end{array}$ & $\begin{array}{l}1801 \\
1961\end{array}$ & $\begin{array}{l}\text { ODS } \\
\text { ODS }\end{array}$ & $02 / 08 / 65$ & 26.500 & $\begin{array}{l}0.9692 \\
0.9562\end{array}$ & $\begin{array}{r}3213,23103 \\
2977.15057\end{array}$ & $\begin{array}{l}18.21 \\
26,06\end{array}$ & $\begin{array}{l}1032,34 \\
1421,77\end{array}$ & 37210 & 32996 & 0221 & 2755 & & $\begin{array}{l}2 \\
2\end{array}$ \\
\hline & 000 & 1962 & ODS & $02 / 08 / 65$ & 27.500 & $0: 9528$ & $2764: 56726$ & $28: 12$ & $1478: 33$ & 34038 & 30076 & $0222^{9}$ & 3237 & 3205 & 2 \\
\hline & 000 & 1961 & ODS & $8 / 65$ & 3.50 & .9780 & 2573 & 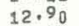 & 65 & 1733 & & & $338_{1}$ & & 2 \\
\hline & 000 & 1961 & ODS & $02 / 08 / 65$ & 29.5 & 0.9651 & 2402.41769 & 0.65 & 1012,34 & 2298 & $2^{89} 0^{7}$ & 16 & 2613 & 2587 & 2 \\
\hline & 000 & 1961 & ODS & $02 / 08 / 65$ & 30.500 & 0.9774 & 2247.46463 & 13.30 & 630.39 & 33564 & 30426 & 0254 & 2513 & 2488 & 2 \\
\hline & 000 & 1961 & ODS & $02 / 08 / 65$ & 31.500 & $\begin{array}{l}0.9593 \\
0.9548\end{array}$ & $\begin{array}{l}03351 \\
36673 \\
3073\end{array}$ & 24.13 & & 9 & & & 2247 & 2225 & 2 \\
\hline & 000 & 1961 & ODS & $02 / 0$ & $32,5,5>0$ & & 191 & 26. & & 237 & $329 / 1$ & & 2293 & 2270 & 2 \\
\hline $\begin{array}{l}u=232 \\
u=232\end{array}$ & 000 & & ODS & $02 / 08 / 65$ & $\begin{array}{l}33,500 \\
34,50\end{array}$ & 0.9517 & $\begin{array}{l}1862,95744 \\
756,52509\end{array}$ & 28.77 & $\begin{array}{l}1241,78 \\
793,38\end{array}$ & $\begin{array}{l}38430 \\
377255\end{array}$ & $\begin{array}{l}33917 \\
33438\end{array}$ & 0,0279 & 1278 & 1265 & 2 \\
\hline & 000 & 1961 & ODS & $02 / 08 / 65$ & 35.500 & $\begin{array}{l}0,9606 \\
0,9606\end{array}$ & 1658,95972 & 23.35 & 951.14 & 36919 & 32886 & 0.0296 & 1917 & $\begin{array}{l}1898 \\
1898\end{array}$ & $\begin{array}{l}2 \\
2\end{array}$ \\
\hline & 000 & 1961 & ODS & $3 / 65$ & 36.5 & 0.9607 & 1569.30305 & 23.28 & 922.07 & 36098 & 32876 & 0304 & 2053 & 2033 & 2 \\
\hline & 000 & 1.961 & ODS & $02 / 08 / 65$ & 37.5 & 0,9623 & 1486.72284 & 22.36 & 862,02 & 37037 & $33_{0} 4 \mathrm{E}$ & 0.0312 & 1529 & 1514 & 2 \\
\hline 232 & 000 & 1961 & ODS & $02 / 08 / 65$ & 38.500 & 0.9689 & 1410.49350 & 18.39 & 690,71 & 36500 & 32796 & 0,0321 & 2290 & 2267 & 2 \\
\hline$U=232$ & 000 & 1961 & ODS & $02 / 08 / 65$ & 39.500 & 0.9570 & 1339.98012 & 25.54 & 934,96 & $\begin{array}{r}36448 \\
-55.07\end{array}$ & 32344 & 0.0329 & 2288 & 2275 & 2 \\
\hline $\begin{array}{l}y=232 \\
y=232\end{array}$ & 000 & 1961 & ODS & $02 / 08 / 65$ & 40.500 & $\begin{array}{l}0.9524 \\
0.9537\end{array}$ & 1274.62520 & 28.34 & 1011,84 & $\begin{array}{l}35197 \\
33319\end{array}$ & $310^{84}$ & 0.0337 & $\begin{array}{l}3567 \\
35516\end{array}$ & $\begin{array}{l}3531 \\
3589\end{array}$ & 2 \\
\hline $\begin{array}{l}=232 \\
u=232\end{array}$ & $\begin{array}{l}000 \\
000\end{array}$ & $\begin{array}{l}1961 \\
1961\end{array}$ & $\begin{array}{l}\text { ODS } \\
\text { ODS }\end{array}$ & $\begin{array}{l}02 / 08 / 65 \\
02 / 08 / 65\end{array}$ & $\begin{array}{l}41.500 \\
42.500\end{array}$ & $\begin{array}{l}0.9537 \\
0.9682\end{array}$ & $\begin{array}{l}1213.9358 \\
1157.48317\end{array}$ & $\begin{array}{l}27.54 \\
18.76\end{array}$ & $\begin{array}{l}959,63 \\
638 ; 41\end{array}$ & $\begin{array}{l}31322 \\
31841\end{array}$ & $\begin{array}{l}27300 \\
28587\end{array}$ & $\begin{array}{l}0.00340 \\
0.0354\end{array}$ & $\begin{array}{l}3510 \\
3512\end{array}$ & $\begin{array}{l}3411 \\
3477\end{array}$ & $\begin{array}{l}2 \\
2\end{array}$ \\
\hline & 000 & 196 & ODS & 02 & 43.5 & 0.9394 & 87726 & 36 & $120^{8}, 06$ & 32193 & $2^{8} 041$ & 0.0362 & 4139 & 4098 & 2 \\
\hline & 000 & & & & & & & & & & & & & & 2 \\
\hline & 000 & 1961 & ODS & & $\begin{array}{l}45.50 \\
46.5\end{array}$ & 0.956 & 1009. & 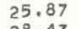 & 822. & 277 & 3 & $\begin{array}{l}0379 \\
0387\end{array}$ & 43 & 4331 & 2 \\
\hline & 000 & 1961 & ODS & $\begin{array}{l}02 / 08 / 65 \\
02 / 8 / 65\end{array}$ & 46.500 & 0.9523 & 966,91132 & 28.43 & 884. & $\begin{array}{r}34630 \\
34374\end{array}$ & $\begin{array}{l}30577 \\
3066^{2}\end{array}$ & 0.0387 & $\begin{array}{l}4408 \\
4598\end{array}$ & $\begin{array}{l}4364 \\
4555 ?\end{array}$ & 2 \\
\hline & 000 & 1961 & $\begin{array}{l}\text { ODS } \\
\text { ODS }\end{array}$ & $02 / 08 / 65$ & 48.500 & $\begin{array}{r}0.9649 \\
0.9625\end{array}$ & 888,0189 & 20.17 & 661. & $\begin{array}{r}54274 \\
34430\end{array}$ & 30718 & $\begin{array}{l}0396 \\
0404 \\
040\end{array}$ & 4579 & 4533 & $\begin{array}{l}2 \\
2\end{array}$ \\
\hline$U=232$ & 000 & $\begin{array}{l}1901 \\
1961\end{array}$ & ODS & $02 / 08 / 65$ & $\begin{array}{r}49.500 \\
49.500\end{array}$ & 0.7023 & 853.26150 & $\begin{array}{l}22,20 \\
23: 80\end{array}$ & 695,29 & 33617 & 29918 & 12 & 4746 & & $\begin{array}{l}2 \\
3\end{array}$ \\
\hline$v=232$ & 000 & 1961 & ODS & $02 / 08 / 65$ & 50,500 & 0.9653 & 819.80354 & 20.50 & 586": & 33983 & 3041.6 & & 47 & 4659 & $2^{2}$ \\
\hline$u=232$ & 000 & 1961 & ODS & & & 0.9 & & & & & & $0.042^{9}$ & & & 2 \\
\hline$U=232$ & 000 & 1961 & ODS & $02 / 08 / 65$ & 52.500 & .9531 & 758.53206 & .90 & 768,42 & 33769 & 29846 & 0.0407 & 4282 & 423 & 2 \\
\hline$U=232$ & 000 & 1961 & ODS & $02 / 08 / 65$ & 53.500 & 0.9676 & 730.4 & 19.16 & 517,74 & 33333 & $2^{99} 04$ & 0,0 & 3818 & 3780 & 2 \\
\hline & 000 & 1861 & Uus & 021 & 54.500 & 0.9602 & 703.4 & 23.61 & $586=3$ & 33322 & $2^{9} 665$ & 0,0463 & 3721 & 3683 & 2 \\
\hline 32 & 000 & 1961 & ODS & $02108 / 65$ & $\begin{array}{l}55.500 \\
56.500\end{array}$ & $\begin{array}{l}0.9620 \\
0.9562\end{array}$ & $\begin{array}{l}678.74490 \\
654.93116\end{array}$ & 22.52 & $\begin{array}{l}586,83 \\
666,32\end{array}$ & $\begin{array}{l}32608 \\
32895\end{array}$ & $\begin{array}{l}29083 \\
29166\end{array}$ & 0.0462 & $\begin{array}{l}3964 \\
3693\end{array}$ & $\begin{array}{l}3924 \\
3656\end{array}$ & 2 \\
\hline & $\begin{array}{l}000 \\
000\end{array}$ & 1961 & ODS & $02 / 08 / 65$ & $\begin{array}{l}20.200 \\
57.500\end{array}$ & 0.9455 & 632.34903 & 32. & 819,02 & 32909 & $\begin{array}{l}2.106 \\
2^{8847}\end{array}$ & & 3128 & & 2 \\
\hline & 000 & 1967 & ODS & $\begin{array}{l}021 \\
021\end{array}$ & 58.5 & 0.9627 & 610.91504 & 32. & 545 & 3206 & 2874 & 0.0487 & 2962 & & $2_{2}^{2}$ \\
\hline & 000 & 196 & ODS & 021 & 59,5 & 0.9555 & 590,55264 & 26.49 & 643.72 & 31742 & $2^{8} \beta_{117}$ & 0,0496 & 2626 & & 2 \\
\hline & 000 & 196 & ODS & & 60 & 0.9548 & 571,19158 & 26 & & 32338 & & Qरen & & & 2 \\
\hline$u=232$ & 000 & 1961 & ODS & 02108 & 61.500 & 0.9476 & 552.76727 & 31.30 & 735,88 & 31883 & $2^{8} 00^{5}$ & 0.0512 & 2194 & 2172 & 2 \\
\hline U. 232 & 000 & 1961 & ODS & $02 / 08 / 65$ & 62.500 & 0.9639 & 535.22022 & 21.36 & 494.08 & 32086 & $2^{8673}$ & 0.0521 & 2089 & 2068 & 2 \\
\hline U. 232 & 000 & 1961 & ODS & 021 & 63.5 & 0.9636 & 518.49563 & 2. & 491,03 & 31679 & $2^{8299}$ & 0.0529 & 1885 & 1866 & 2 \\
\hline & 000 & & ODS & & & 0 & & & & 32 & $2^{85} 5_{14}^{4}$ & 0537 & & 1828 & 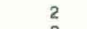 \\
\hline 232 & 000 & 1961 & ODS & 02108 & 65.500 & 0.95 & 487.31 & 27.41 & $609.1 \frac{11}{9}$ & 3178 & $2^{8}$ & ( & 1710 & 1692 & 2 \\
\hline 32 & 000 & 1961 & ODS & $02 / 08$ & 66.500 & 0,9484 & & & & & & 62 & & $\begin{array}{l}1384 \\
1339\end{array}$ & 2 \\
\hline 32 & 000 & $\begin{array}{l}1961 \\
1961\end{array}$ & $\begin{array}{l}\text { ODS } \\
\text { ODS }\end{array}$ & $\begin{array}{r}02 / 08 / 65 \\
02 / 08 / 65\end{array}$ & $\begin{array}{l}67.500 \\
68.500\end{array}$ & $\begin{array}{l}0.9473 \\
0.9045\end{array}$ & $\begin{array}{l}458.06507 \\
445.56535\end{array}$ & $\begin{array}{l}31.50 \\
58.36\end{array}$ & $\begin{array}{r}674,74 \\
1231.99\end{array}$ & $\begin{array}{l}31034 \\
30954\end{array}$ & $\begin{array}{l}27258 \\
25954\end{array}$ & $\begin{array}{l}0.0202 \\
0.0571\end{array}$ & $\begin{array}{l}1453 \\
1293\end{array}$ & $\begin{array}{l}1439 \\
1280\end{array}$ & ${ }^{2}$ \\
\hline & 000 & 1961 & ODS & & 69.5 & $\begin{array}{l}0.7045 \\
0.9535\end{array}$ & $\begin{array}{r}445.50535 \\
432.83557\end{array}$ & & $\begin{array}{l}1231, \\
575,\end{array}$ & 30 & 26460 & 0.0579 & & 1284 & 2 \\
\hline & 000 & 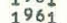 & ODS & & 70. & 0.9449 & 420.64 & 32.9 & & & & & & & 2 \\
\hline & 000 & 96 & ODS & & & & & & & & & & & & 2 \\
\hline 232 & 000 & 196 & ODS & $02 / 08 / 65$ & 72.500 & 0,9597 & 397.75581 & 23.91 & 476,88 & 29055 & 25848 & 0.0604 & 1115 & 1104 & 2 \\
\hline$u=232$ & 000 & 1961 & ODS & $02 / 08 / 65$ & 73.500 & 0,9588 & 38 & 24,46 & 48 & 27493 & 244 & 0612 & 11 & 1144 & 2 \\
\hline & 000 & 1961 & ODS & & 0 & 0 & & & & & & & 1007 & & 2 \\
\hline 232 & 000 & 1961 & ODS & $02 \%$ & $\begin{array}{l}75.500 \\
76.500\end{array}$ & $\begin{array}{l}0.9639 \\
0.9464\end{array}$ & $\begin{array}{l}366.77 \\
357.24\end{array}$ & 21.35 & $40^{8}$ & 214 & $1^{9}$ & 0.0 & 1111 & 1100 & 2 \\
\hline $\begin{array}{l}232 \\
232\end{array}$ & $\begin{array}{l}000 \\
000\end{array}$ & $\begin{array}{l}1961 \\
1961\end{array}$ & ODS & $\begin{array}{l}0220 \\
02108\end{array}$ & $\begin{array}{l}76.500 \\
77.500\end{array}$ & $\begin{array}{l}0.9464 \\
0,9358\end{array}$ & 35 & & $0_{0}^{4}$ & & $\begin{array}{l}1465 \% \\
1096^{3}\end{array}$ & 6 & 1135 & $\begin{array}{l}1143 \\
990\end{array}$ & 2 \\
\hline & 000 & 170 & ODS & & 78 & 0 & & & & & & 54 & & & 2 \\
\hline & 000 & 1961 & ODS & & & $0.5 \quad-5$ & & & & & & & 10 & 1081 & 2 \\
\hline & 000 & 1961 & ODS & & 80.5 & 0 & & & 84 & & ${ }_{189}$ & & 11 & 1162 & $=$ \\
\hline & 000 & 1961 & ODS & & & & & & & & & & 10 & 1074 & 2 \\
\hline 232 & 000 & 96. & ODS & $02 / 08 / 65$ & 82. & 0.9429 & 307.17414 & 34.20 & 599,36 & 26889 & 23500 & 0.0687 & & 968 & 2 \\
\hline 232 & 000 & 1961 & ODS & $02 / 08 / 65$ & 83.500 & 0.9335 & 299 & 39.98 & 692.24 & 27498 & 23794 & & 1041 & & 2 \\
\hline & 0 & 196 & ODS & & & & & & & & $\begin{array}{l}25100 \\
25546\end{array}$ & & $\begin{array}{l}1141 \\
1274\end{array}$ & $\begin{array}{l}1129 \\
1261\end{array}$ & 2 \\
\hline ? & 000 & 1961 & ODS & & & & & & & & 26411 & & 11 & & 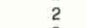 \\
\hline & 000 & 196 & ODS & & 0 & & & & & & & & & & \\
\hline & 000 & $19 t>-1$ & ODS & & 88.5 & & & & & & $2^{5}$ & 0.0786 & & & \\
\hline & 000 & 1 & DS & & & & & & & & & ${ }_{4}$ & & & \\
\hline 2 & 000 & 1961 & $\begin{array}{l}\text { ODS } \\
\text { ODS }\end{array}$ & $\begin{array}{l}02 \\
02\end{array}$ & 9. & 0.927 & $\begin{array}{l}25 \\
25 \\
29\end{array}$ & & & $\begin{array}{l}29 \\
29\end{array}$ & $\begin{array}{l}20961 \\
25595\end{array}$ & 62 & $\begin{array}{l}1354 \\
1342\end{array}$ & $\begin{array}{l}1345 \\
1329\end{array}$ & 2 \\
\hline 232 & 000 & $\begin{array}{l}1701 \\
1961\end{array}$ & ODS & U2/08/65 & 92.500 & 0.9524 & $244.340_{1} 7$ & $\begin{array}{l}43.40 \\
28.35\end{array}$ & 443,09 & 30144 & $2^{66} 60^{5}$ & 0.0771 & 1337 & 1323 & 2 \\
\hline
\end{tabular}




\begin{tabular}{|c|c|c|c|c|c|c|c|c|c|c|c|c|}
\hline ENTIFICATION & DATE & DELAY & TRANS. & ENERGY & $x$-SEC & $\begin{array}{l}x=S E C \\
\text { SOSET }\end{array}$ & $\mathrm{cos}$ & & $\begin{array}{l}\text { FRAC, } \\
\text { FE OCT }\end{array}$ & $\begin{array}{l}\text { FAST } \\
\text { OPEN }\end{array}$ & KGS & NO, PTS \\
\hline $\begin{array}{llll}000 & 1961 \\
0 & 0 D S & 1961 \\
005\end{array}$ & $\begin{array}{l}02 / 08 / 65 \\
20 / 18 / 65\end{array}$ & $\begin{array}{l}93.500 \\
94.550\end{array}$ & $\begin{array}{l}0.9681 \\
0.9726\end{array}$ & $\begin{array}{l}239.14942 \\
234.11433\end{array}$ & $\begin{array}{l}18,82 \\
16.14\end{array}$ & $\begin{array}{l}290,09 \\
246,99\end{array}$ & $\begin{array}{l}29541 \\
29951 \\
29951\end{array}$ & $\begin{array}{l}2650 \% \\
27000\end{array}$ & $\begin{array}{l}0,0779 \\
0,0787\end{array}$ & $\begin{array}{l}1376 \\
1296\end{array}$ & $\begin{array}{l}1362 \\
1283\end{array}$ & \\
\hline 0001961 ODS & & 95.500 & 0.9600 & $\begin{array}{l}229.23758 \\
224.51115\end{array}$ & $\begin{array}{l}10.14 \\
23.74 \\
21.85\end{array}$ & & $\begin{array}{l}2991 \\
29885 \\
3\end{array}$ & $\begin{array}{l}2600 \\
26590 \\
2798\end{array}$ & $\begin{array}{l}0,0796 \\
0.0804\end{array}$ & $\begin{array}{l}1323 \\
1323\end{array}$ & $\begin{array}{l}1309 \\
1309\end{array}$ & \\
\hline $\left.\begin{array}{lll}000 & 1961 & 0 D S \\
00 & 1961 & \text { ODS }\end{array}\right]$ & $\begin{array}{l}02 / 08 / 15 \\
02 / 08 / 65\end{array}$ & $\begin{array}{l}96.500 \\
97.500\end{array}$ & $\begin{array}{l}0.9698 \\
0.9423\end{array}$ & 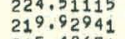 & $\begin{array}{l}\frac{17.85}{34.52} \\
34.5\end{array}$ & $\begin{array}{l}267.51 \\
5_{11}^{26}: 95\end{array}$ & $\begin{array}{l}30259 \\
29930 \\
29930\end{array}$ & $\begin{array}{l}{ }_{2}^{2 / 1196} \\
2^{6141}\end{array}$ & $\begin{array}{l}0.0804 \\
0.0812\end{array}$ & $\begin{array}{l}1341 \\
1214\end{array}$ & $\begin{array}{l}1328 \\
1202\end{array}$ & \\
\hline $\begin{array}{l}32 \\
322000 \\
3\end{array}$ & $\begin{array}{l}02 / 08 / 65 \\
02 / 0865\end{array}$ & $\begin{array}{l}98.500 \\
99.550\end{array}$ & $\begin{array}{l}0.9201 \\
0.9552\end{array}$ & $\begin{array}{l}215.48651 \\
211.1769\end{array}$ & $\begin{array}{l}48.44 \\
26.63 \\
26.63\end{array}$ & 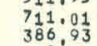 & $\begin{array}{l}29967 \\
29872 \\
29872\end{array}$ & $\begin{array}{l}25553 \\
26446 \\
26446\end{array}$ & $\begin{array}{l}0.0821 \\
0.0829\end{array}$ & $\begin{array}{l}1396 \\
1256\end{array}$ & $\begin{array}{l}1 \\
1.362 \\
1 \\
1353\end{array}$ & \\
\hline 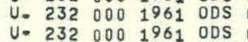 & $\begin{array}{l}02 / 08,65 \\
02 / 185\end{array}$ & 100.500 & 0.9478 & $\begin{array}{l}206.99527 \\
202.93604\end{array}$ & $\begin{array}{l}31.18 \\
31.16\end{array}$ & 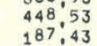 & $\begin{array}{l}29656 \\
28551 \\
28551\end{array}$ & $\begin{array}{l}26050 \\
25865 \\
2586\end{array}$ & $\begin{array}{l}0.0837 \\
0.0846\end{array}$ & $\begin{array}{l}1292 \\
1292 \\
1352\end{array}$ & $\begin{array}{l}1279 \\
1338\end{array}$ & \\
\hline 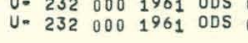 & $\begin{array}{l}02 / 108 / 65 \\
02 / 08 / 65\end{array}$ & 102.500 & 0.9525 & 198.99622 & $\begin{array}{l}28,30 \\
2.30\end{array}$ & & & $\begin{array}{l}25845 \\
2580\end{array}$ & & 1192 & $118_{0}$ & \\
\hline $\begin{array}{llll}232 & 000 & 1961 & 005\end{array}$ & $02 / 08 / 65$ & $\begin{array}{l}103.500 \\
1.50\end{array}$ & 0,8919 & $\begin{array}{l}195.16943 \\
.91 .45203\end{array}$ & 66.52 & $\begin{array}{r}929 \cdot 24 \\
1548.63\end{array}$ & $\begin{array}{l}29210 \\
29445\end{array}$ & $\begin{array}{l}24144 \\
22549\end{array}$ & 0.0862 & $\begin{array}{l}1178 \\
1234\end{array}$ & 1166 & \\
\hline 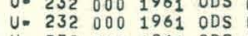 & & $\begin{array}{l}104.500 \\
105,500\end{array}$ & $\begin{array}{l}0.8249 \\
0,8554\end{array}$ & 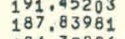 & $\begin{array}{r}111.92 \\
90.77\end{array}$ & $\begin{array}{l}\frac{1548}{154.63} .04 \\
124.04\end{array}$ & $\begin{array}{l}29445 \\
29003\end{array}$ & 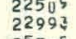 & $\begin{array}{l}0,0671 \\
0,0879\end{array}$ & $\begin{array}{l}1234 \\
1185\end{array}$ & ${ }_{1173}^{221}$ & \\
\hline 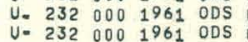 & $\begin{array}{l}02 / 08 / 15 \\
02 / 081 / 65\end{array}$ & $\begin{array}{l}106.500 \\
107500\end{array}$ & $\begin{array}{l}0.9327 \\
0.9559\end{array}$ & $\begin{array}{l}184,32886 \\
180.91543\end{array}$ & $\begin{array}{l}40,48 \\
26,19\end{array}$ & $\begin{array}{l}549.62 \\
352: 33\end{array}$ & $\begin{array}{l}29506 \\
28924 \\
28924\end{array}$ & $\begin{array}{l}25505 \\
25625\end{array}$ & $\begin{array}{l}0,0887 \\
0,0896\end{array}$ & $\begin{array}{l}1030 \\
1160\end{array}$ & $\begin{array}{l}1020 \\
1148\end{array}$ & \\
\hline $\begin{array}{rlll}32 & 000 & 1961 & \text { ODS } \\
32 & 000 & 1961 & \text { OD }\end{array}$ & $\begin{array}{l}02 / 08 / 65 \\
02 / 08 / 65\end{array}$ & $\begin{array}{l}108.500 \\
109.500\end{array}$ & $\begin{array}{l}0.9529 \\
0.9580\end{array}$ & $\begin{array}{l}177.59595 \\
174.36701\end{array}$ & $\begin{array}{l}28.06 \\
24.92\end{array}$ & $\begin{array}{l}373,94 \\
329: 02\end{array}$ & 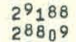 & & & $\begin{array}{r}953 \\
1080\end{array}$ & $\begin{array}{r}944 \\
1069\end{array}$ & \\
\hline $\begin{array}{l}1961 \text { o s } \\
1961 \text { ODS }\end{array}$ & $\begin{array}{l}0210865 \\
02 / 0865\end{array}$ & $\begin{array}{l}109.500 \\
110.500 \\
1100\end{array}$ & $\begin{array}{l}0,9560 \\
0.9470 \\
0.9618\end{array}$ & 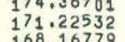 & $\begin{array}{l}24.02 \\
31,66 \\
3.56\end{array}$ & 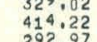 & $\begin{array}{ll}29009 \\
29043\end{array}$ & $\begin{array}{ll} & \end{array}$ & $\begin{array}{l}0.0912 \\
0.0921 \\
0.0229\end{array}$ & $\begin{array}{l}1000 \\
1008\end{array}$ & $\begin{array}{l}1097 \\
997\end{array}$ & \\
\hline 1320001961 ODS & 021 & 112.50 & , 9459 & ${ }_{165}, 19143$ & 2.34 & 415,06 & 29670 & 26008 & 0.0937 & 886 & $\begin{array}{l}877 \\
877\end{array}$ & \\
\hline $\begin{array}{llll}32 & 000 & 1961 & \text { ODS } \\
3 & 0.00\end{array}$ & $02 / 08 / 65$ & 113.500 & 0,9222 & 162.29339 & 47.07 & 9,58 & 28962 & 24758 & 0.0946 & 875 & 66 & \\
\hline $\begin{array}{l}1991 \text { ODS } \\
1961 \text { ODS }\end{array}$ & $\begin{array}{l}02 / 08 / 155 \\
02 / 08 / 65\end{array}$ & $\begin{array}{l}114.500 \\
115.500\end{array}$ & $\begin{array}{l}0.8564 \\
0,8888\end{array}$ & 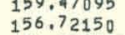 & $\begin{array}{l}90.11 \\
68.50\end{array}$ & 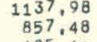 & $\begin{array}{l}{ }_{28585}^{29237} \\
2^{8585}\end{array}$ & $\begin{array}{l}{ }_{2}^{23203} 2354 \\
2354\end{array}$ & $\begin{array}{l}0.0954 \\
0.0962\end{array}$ & $\begin{array}{l}918 \\
913\end{array}$ & $\begin{array}{l}909 \\
904\end{array}$ & \\
\hline & & $\begin{array}{l}116 . \\
117\end{array}$ & $\begin{array}{l}9093 \\
8985 \\
8985\end{array}$ & $\begin{array}{l}\begin{array}{l}54.04253 \\
151.43171\end{array} \\
151\end{array}$ & & $0_{0}^{0}-2-2$ & & & 871 & $\begin{array}{l}794 \\
802 \\
802\end{array}$ & $\begin{array}{l}786 \\
794\end{array}$ & \\
\hline of & 270 & 118.500 & 8626 & 148,88668 & 43. & 30.46 & & & 0,0987 & 793 & 785 & \\
\hline 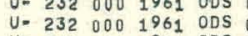 & $\begin{array}{l}08765 \\
108 / 65\end{array}$ & $\begin{array}{l}119.500 \\
120.500\end{array}$ & 10026 & $\begin{array}{l}140.40728 \\
143.98540\end{array}$ & $\begin{array}{c}65 . \\
151\end{array}$ & $\begin{array}{l}6 \\
6\end{array}$ & & & ${ }_{04}^{96}$ & $\begin{array}{l}692 \\
761 \\
761\end{array}$ & 要65 & \\
\hline 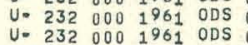 & $\begin{array}{l}02 / 08 / 15 \\
02105 \\
0010865\end{array}$ & $\begin{array}{l}121.500 \\
122.500\end{array}$ & $\begin{array}{l}0.8843 \\
0,9671\end{array}$ & $\begin{array}{l}141.62502 \\
139.32221\end{array}$ & $\begin{array}{l}71.46 \\
19.42\end{array}$ & $\begin{array}{l}850,37 \\
229 ; 25 \\
229\end{array}$ & $\begin{array}{l}27540 \\
26506\end{array}$ & $\begin{array}{l}2256 \mathrm{k} \\
2375\end{array}$ & $\begin{array}{l}0.1012 \\
0,1021\end{array}$ & 659 & 653 & \\
\hline 0196 & $02 / 08 / 65$ & 123.500 & .9544 & 137.07512 & 27.11 & 317.43 & ${ }^{26} 6_{142}$ & ${ }^{23}{ }_{121}$ & 0,1029 & 738 & 701 & \\
\hline & & $\begin{array}{l}\text { 124: } \\
125:\end{array}$ & $\begin{array}{l}0 \\
0\end{array}$ & 132.74100 & & & & & & $\begin{array}{l}154 \\
604\end{array}$ & 658 & \\
\hline 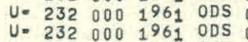 & $\begin{array}{l}02108 / 15 \\
02 / 0865\end{array}$ & $\begin{array}{l}126.500 \\
127500\end{array}$ & $\begin{array}{l}0.7600 \\
0.8176\end{array}$ & $\begin{array}{l}130.65063 \\
128.6094\end{array}$ & $\begin{array}{l}159.57 \\
117.03\end{array}$ & $\begin{array}{l}1823.88 \\
132.24\end{array}$ & $\begin{array}{l}25986 \\
26144 \\
2614\end{array}$ & $\begin{array}{l}18295 \\
19848 \\
1980\end{array}$ & $\begin{array}{l}0: 1054 \\
0: 1062\end{array}$ & $\begin{array}{l}672 \\
621\end{array}$ & $\begin{array}{l}665 \\
615\end{array}$ & \\
\hline $32000196100 \mathrm{~s}$ & $\begin{array}{l}02108 / 65 \\
02108\end{array}$ & $\begin{array}{l}128.500 \\
129 \\
1200\end{array}$ & $\begin{array}{l}0.6980 \\
0.9263\end{array}$ & $\begin{array}{l}126.01533 \\
124.66743\end{array}$ & $\begin{array}{r}62.57 \\
645 \\
445\end{array}$ & $\begin{array}{l}704.05 \\
4968 \\
498\end{array}$ & $\begin{array}{l}27501 \\
27787\end{array}$ & $\begin{array}{l}22883 \\
23850\end{array}$ & $\begin{array}{l}0.1071 \\
0.1079\end{array}$ & $\begin{array}{l}707 \\
807\end{array}$ & $\begin{array}{l}700 \\
799\end{array}$ & \\
\hline $\begin{array}{l}1961 \text { ons } \\
1961005\end{array}$ & $\begin{array}{l}02 / 08,65 \\
02 / 10865\end{array}$ & $\begin{array}{l}130.500 \\
131.500\end{array}$ & $\begin{array}{l}0,9546 \\
0,9593\end{array}$ & $\begin{array}{l}122.76416 \\
120.90410\end{array}$ & $\begin{array}{l}27.03 \\
24.16 \\
24.16\end{array}$ & $\begin{array}{l}299: 54 \\
295 ; 69\end{array}$ & $\begin{array}{l}2_{28191}^{28196} \\
z_{2866}\end{array}$ & $\begin{array}{l}24935 \\
25125 \\
25125\end{array}$ & $\begin{array}{l}0.1087 \\
0.1096\end{array}$ & $\begin{array}{l}655 \\
602\end{array}$ & $\begin{array}{l}649 \\
656\end{array}$ & \\
\hline 2320001961 ODS & $02 / 08 / 65$ & 132.500 & 0.9768 & 119.08602 & 13.63 & 148,76 & 27941 & 25290 & $0.110^{4}$ & 738 & 730 & \\
\hline $\begin{array}{llll}000 & 1961 & \text { ODS } \\
0 & 1961 & \text { ODS }\end{array}$ & $02 / 08 / 65$ & $\begin{array}{l}133.500 \\
134\end{array}$ & 9669 & $\begin{array}{r}117.30864 \\
1.550 .57076\end{array}$ & 19.5 & $\begin{array}{l}211.99 \\
71.40\end{array}$ & 35 & $\begin{array}{l}24490 \\
23119\end{array}$ & 0.1112 & $\begin{array}{l}831 \\
831 \\
819\end{array}$ & 822 & \\
\hline & & 135. & 20 & 列3,87122 & 要 & & & & 0. & 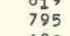 & $\begin{array}{l}711 \\
787\end{array}$ & \\
\hline 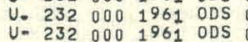 & $\begin{array}{l}02 / 08,165 \\
02 / 0865\end{array}$ & $\begin{array}{l}136,500 \\
1375500\end{array}$ & $\begin{array}{l}0,9109 \\
0,8173 \\
0,817\end{array}$ & $\begin{array}{l}112,20808 \\
11058269\end{array}$ & $\begin{array}{r}54.22 \\
117.28\end{array}$ & $\begin{array}{r}574,37 \\
1233 ; 31\end{array}$ & $\begin{array}{l}28110 \\
27813 \\
27813\end{array}$ & $\begin{array}{l}23726 \\
21068\end{array}$ & $\begin{array}{l}0: 1137 \\
0: 1146\end{array}$ & $\begin{array}{l}{ }_{802}^{692} \\
8_{03}\end{array}$ & $\begin{array}{l}685 \\
795\end{array}$ & \\
\hline 0001961 ODS & $\begin{array}{c}65 \\
55 \\
\end{array}$ & $\begin{array}{l}138.500 \\
139.500\end{array}$ & $\begin{array}{l}0.0612 \\
0,7458\end{array}$ & $\begin{array}{l}10^{8} 8,99159 \\
10^{7} 743459\end{array}$ & $\begin{array}{l}240.45 \\
170.52\end{array}$ & $\begin{array}{l}2510,33 \\
1767: 49\end{array}$ & $\begin{array}{l}696 \\
526\end{array}$ & & $\begin{array}{l}54 \\
62 \\
62\end{array}$ & $\begin{array}{l}843 \\
{ }_{816}\end{array}$ & $\begin{array}{l}835 \\
808\end{array}$ & \\
\hline $\begin{array}{l}0001961 \\
000 \\
000 \\
0061961\end{array}$ & $\begin{array}{l}02 / 08 / 65 \\
02 / 0865\end{array}$ & $\begin{array}{l}140.500 \\
141 \\
1400\end{array}$ & $\begin{array}{l}0,8708 \\
0,8987\end{array}$ & $\begin{array}{l}105.91072 \\
104.41903\end{array}$ & $\begin{array}{l}80.42 \\
60.210 \\
62.10\end{array}$ & $\begin{array}{l}827.65 \\
624 ; 58\end{array}$ & $\begin{array}{l}28118 \\
27118 \\
27268\end{array}$ & & 0.1171 & $\begin{array}{l}834 \\
795\end{array}$ & $\begin{array}{l}826 \\
787\end{array}$ & \\
\hline 0001961 ODS & $\begin{array}{l}2 / 08 / 65 \\
2,105\end{array}$ & $\begin{array}{l}14 \\
14\end{array}$ & & $\begin{array}{l}102,95864 \\
102,954\end{array}$ & & & & 22 & & 795 & 787 & \\
\hline 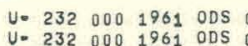 & $02 / 08 / 65$ & $\begin{array}{l}143.500 \\
14450\end{array}$ & 0.9625 & $\begin{array}{l}101.52868 \\
100.12830\end{array}$ & 2 & & $\begin{array}{l}27491 \\
27075\end{array}$ & & & 798 & 790 & \\
\hline & & 14 & $\begin{array}{l}9810 \\
9596 \\
9596\end{array}$ & $\begin{array}{r}98,75670 \\
97.4130\end{array}$ & $\begin{array}{l}11.13 \\
23.99\end{array}$ & $\begin{array}{l}0.56 \\
6.74\end{array}$ & & & 2 & $\begin{array}{l}791 \\
858\end{array}$ & $\begin{array}{l}783 \\
849\end{array}$ & \\
\hline $00 \mathrm{~s}$ & $02 / 08 / 65$ & 147500 & 0.9670 & $\begin{array}{l}96.09671 \\
94.09683\end{array}$ & 19.52 & 191,39 & $\begin{array}{r}27190 \\
27199\end{array}$ & 24368 & 0.1229 & 907 & 898 & \\
\hline $\begin{array}{l}\text { ODS } \\
\text { oDs }\end{array}$ & $\begin{array}{l}0210875 \\
02108 / 65\end{array}$ & $\begin{array}{l}148.500 \\
149.500\end{array}$ & $\begin{array}{l}0.873 \\
0.971\end{array}$ & $\begin{array}{l}74.00627 \\
93,5427 \\
93230\end{array}$ & 16. & $\begin{array}{l}150.60 \\
160.72\end{array}$ & 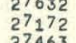 & $\begin{array}{ll}24 \\
24465 \\
2405\end{array}$ & & $\begin{array}{l}824 \\
854\end{array}$ & $\begin{array}{l}817 \\
845\end{array}$ & \\
\hline 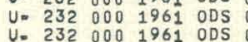 & $\begin{array}{l}02 / 08 / 65 \\
02 / 0865\end{array}$ & $\begin{array}{l}151.500 \\
152.500\end{array}$ & $\begin{array}{l}0.9775 \\
0.9826\end{array}$ & $\begin{array}{l}91.08928 \\
89.8959\end{array}$ & $\begin{array}{l}10.20 \\
10.20\end{array}$ & $\begin{array}{l}126.54 \\
1260 \\
963\end{array}$ & $\begin{array}{l}27316 \\
27725\end{array}$ & $\begin{array}{l}24737 \\
25240\end{array}$ & $\begin{array}{l}1262 \\
1271 \\
121\end{array}$ & 1081 & $\begin{array}{r}949 \\
1070\end{array}$ & \\
\hline$s$ & $02 / 08 / 65$ & 153.50 & .9658 & 88.73108 & & 0. & & & 79 & 999 & $985 \mathrm{~s}>\mathrm{l}$ & \\
\hline & & & & $\begin{array}{l}87.58 \\
86.46\end{array}$ & & & & & & & & \\
\hline $\begin{array}{l}196 \\
196\end{array}$ & $\begin{array}{l}02 / 08 / 65 \\
02 / 1 / 65\end{array}$ & $\begin{array}{l}155500 \\
156.500\end{array}$ & $\begin{array}{l}0.9835 \\
0.9711\end{array}$ & $\begin{array}{l}85.36186 \\
85.6186\end{array}$ & 17. & 157.40 & 27636 & $\begin{array}{l}24 \\
24 \\
24\end{array}$ & $\begin{array}{l}0.1290 \\
0.1304\end{array}$ & $\begin{array}{l}1105 \\
1159\end{array}$ & $\begin{array}{l}10948 \\
1148\end{array}$ & \\
\hline 196 & & 年 & $\begin{array}{l}.9655 \\
9668\end{array}$ & $\begin{array}{l}84,2134 \\
83,22121\end{array}$ & & & & & & 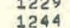 & $\begin{array}{l}1217 \\
{ }_{1232}^{32}\end{array}$ & \\
\hline & $02 / 08$ & 159.5 & & 82,18095 & & & & & & & & \\
\hline 2320001761 & $\begin{array}{l}2 / 1 / 8165 \\
2 / 108 / 65\end{array}$ & $\begin{array}{l}160.500 \\
161.500\end{array}$ & $\begin{array}{l}0.968 \\
0.950\end{array}$ & 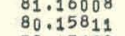 & $\begin{array}{l}18,0 \\
29\end{array}$ & $\begin{array}{l}169 \\
260\end{array}$ & & $\begin{array}{l}24 \\
24 \\
24\end{array}$ & & $\begin{array}{l}13351 \\
1329\end{array}$ & 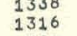 & \\
\hline & & & & & & & & & & & & \\
\hline 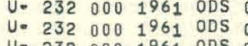 & $\begin{array}{l}02 / 08 / 15 \\
02 / 0^{0} / 65\end{array}$ & $\begin{array}{l}163.500 \\
164.500\end{array}$ & 0,9430 & $\begin{array}{l}78.209055 \\
77.26107\end{array}$ & 34. & & & & & 1437 & 1 & \\
\hline $19 t$ & & 16 & 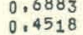 & $\begin{array}{l}76.33023 \\
75,41610\end{array}$ & $\begin{array}{l}217: \\
461:\end{array}$ & 196 & & & & $\begin{array}{l}1441 \\
1484\end{array}$ & $\begin{array}{l}1426 \\
1469\end{array}$ & \\
\hline & & & & 74.51830 & 78 & & & & & & 13 & \\
\hline $\begin{array}{ll}2332 & 0 \\
232 & 0 \\
0\end{array}$ & $\begin{array}{l}021081 / 15 \\
02 / 108\end{array}$ & $\begin{array}{l}168.500 \\
169.500\end{array}$ & $\begin{array}{l}7803 \\
9180\end{array}$ & $\begin{array}{l}73.63643 \\
72.77013\end{array}$ & $\begin{array}{l}144 . \\
49\end{array}$ & $\begin{aligned} \begin{array}{r}2331.43 \\
424,43\end{array} & \end{aligned}$ & & & & $\begin{array}{l}13 \\
13\end{array}$ & $\begin{array}{l}1380 \\
1357\end{array}$ & \\
\hline & & $\begin{array}{l}170.500 \\
171: 500\end{array}$ & & $\begin{array}{l}71,01 \\
71: 08\end{array}$ & & & & & & & & \\
\hline & $02 \%$ & 172.500 & & 70.26 & & & & & & & & \\
\hline $\begin{array}{llll}000 & 1961 \text { ODS }\end{array}$ & $02 / 08 / 65$ & 173.500 & 0.9788 & $\begin{array}{l}69,4534 \\
68.6596\end{array}$ & 12. & $\begin{array}{l}103,60 \\
120.97\end{array}$ & & & & & 13 & \\
\hline 005 & 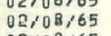 & $\begin{array}{l}2175.5 \\
175\end{array}$ & 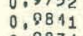 & 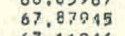 & 0. & & & & & 1231 & $\begin{array}{l}1210 \\
1210\end{array}$ & \\
\hline & $\begin{array}{l}02 / 08 \\
02 / 08\end{array}$ & $\begin{array}{l}1776,500 \\
177: 500\end{array}$ & & (6):3583 & & & & & & & & \\
\hline & & & & $\begin{array}{ll}65.6169 \\
6.6180\end{array}$ & & & & & & & & \\
\hline & & & & 年 & & & & & & & & \\
\hline & & & & $63.4^{6}-2$ & & & & & & & & \\
\hline & $2 / 08 / 65$ & & 9782 & 62,7 & , & & & & & & & \\
\hline 1961 ODS & $\begin{array}{l}02 / 08 / 65 \\
02 / 65\end{array}$ & $\begin{array}{l}183.500 \\
184500\end{array}$ & $\begin{array}{l}.9618 \\
9685\end{array}$ & $\begin{array}{l}62,08982 \\
61.41859\end{array}$ & 22. & 178.4 & & 231 & & 11 & 113 & \\
\hline & & & & & & & & & & & & \\
\hline 32 & & $\begin{array}{l}186.5 \\
187.5\end{array}$ & 0.931 & $\begin{array}{l}\begin{array}{l}60.10036 \\
59.46896\end{array} \\
59.468\end{array}$ & $\begin{array}{l}41.7 \\
38\end{array}$ & 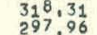 & 58 & 年 & 年 & $\frac{112}{104}$ & $\begin{array}{l}1110 \\
1037\end{array}$ & \\
\hline $\begin{array}{ll}32 \\
32 \\
0\end{array}$ & $\begin{array}{l}02108, \\
02108 \\
021\end{array}$ & $\begin{array}{l}188,500 \\
189: 500\end{array}$ & $\begin{array}{l}0: 9690 \\
0: 9680\end{array}$ & 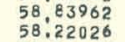 & 3 & & & & & $\begin{array}{l}11 \\
10\end{array}$ & $\begin{array}{l}1126 \\
1063\end{array}$ & \\
\hline 3200019610 & 0210 & & & & & & & & & & & \\
\hline & $\begin{array}{l}02 / 108 / 65 \\
0.05\end{array}$ & & & .01052 & & & & & & & & \\
\hline
\end{tabular}




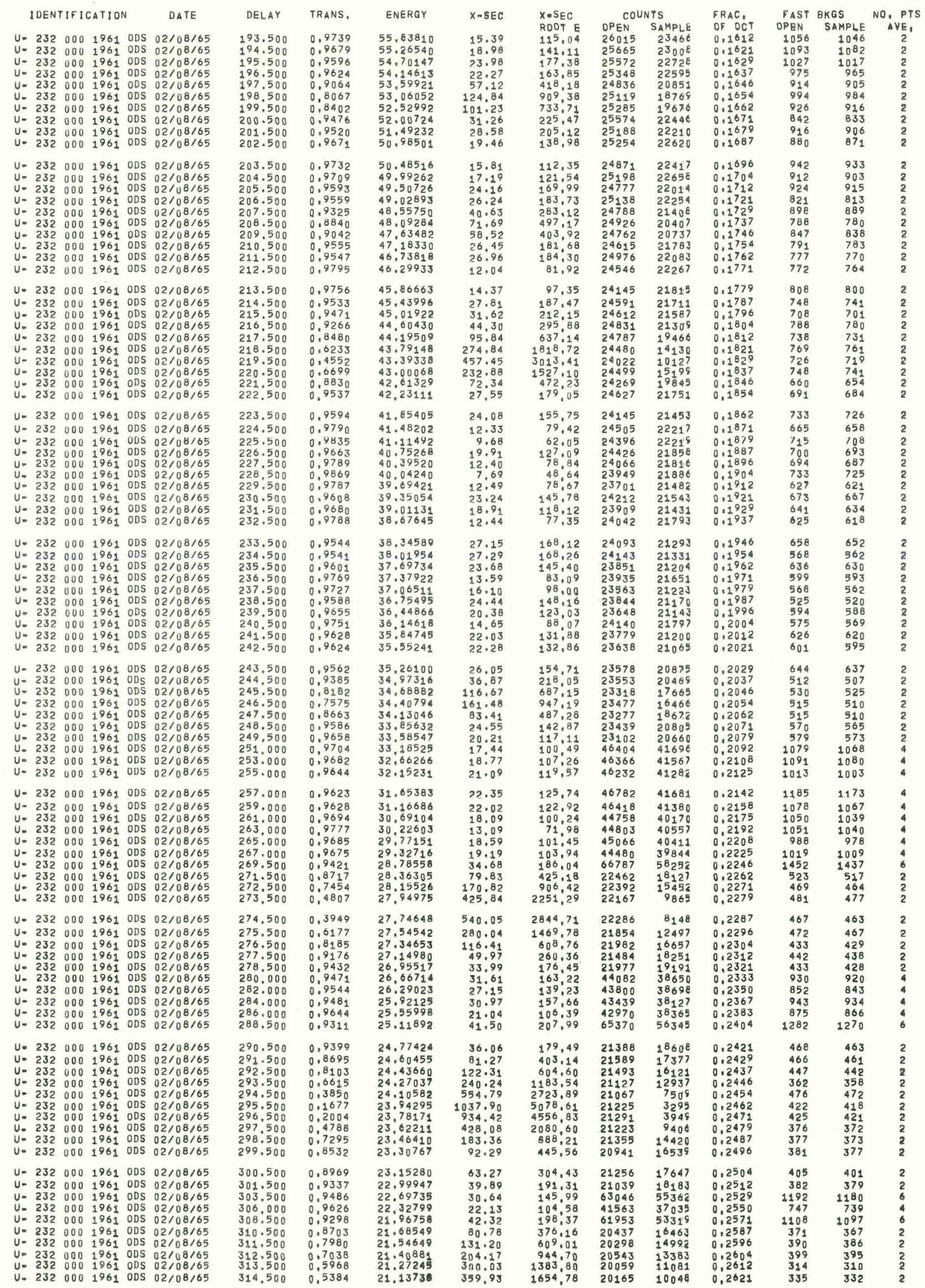




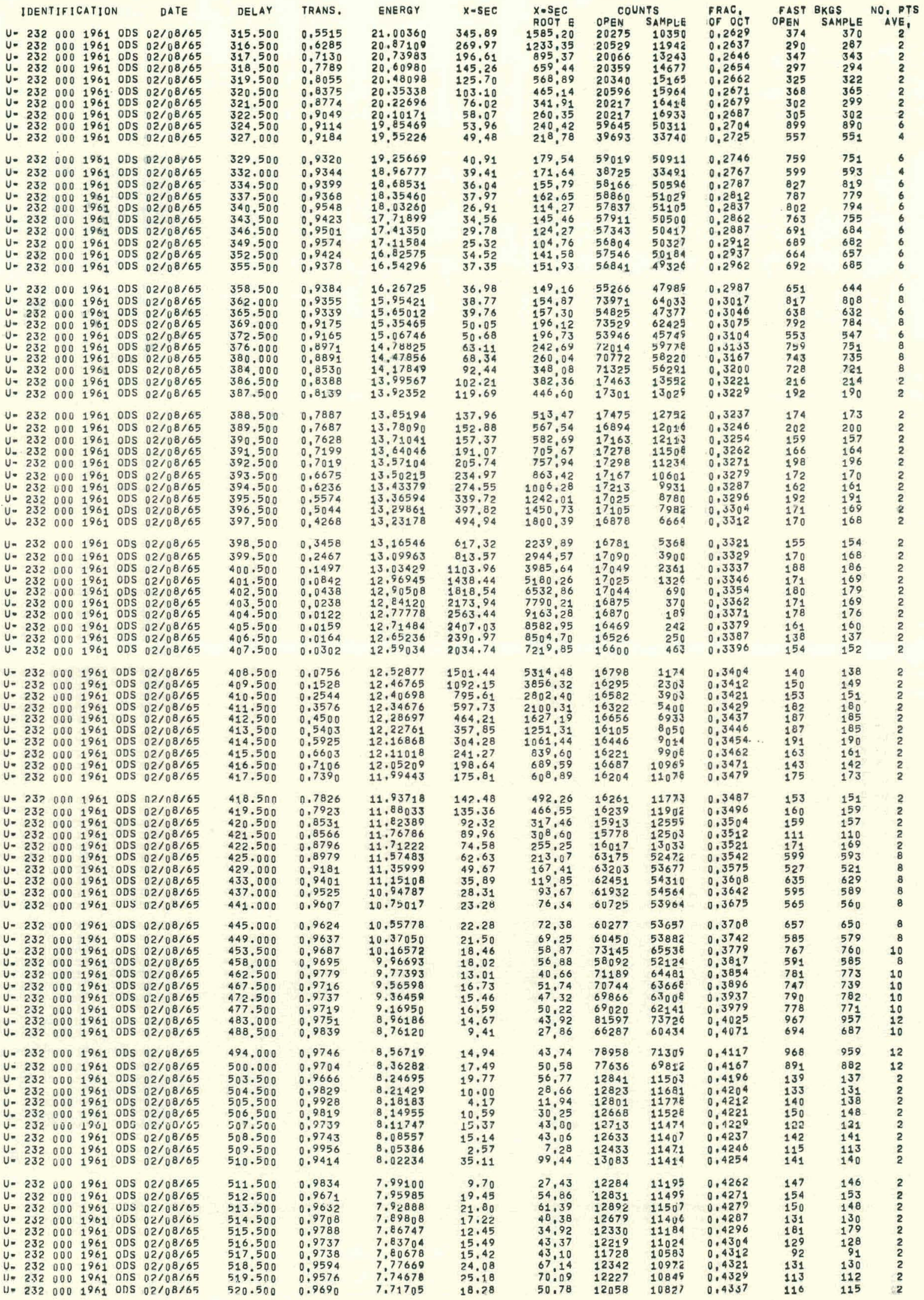




\begin{tabular}{|c|c|c|c|c|c|c|c|c|c|c|c|}
\hline [DEN] & TIFI & CATION & & DATE & DELAY & TRANS. & ENERGY & $x-$ SEC & $X \odot S E C$ & $\mathrm{CO}$ & JNTS \\
\hline v. 232 & 000 & 1961 & ODS & $02 / 08 / 65$ & 527.500 & 0,9746 & 7,68748 & 14.98 & $\begin{array}{r}\text { ROOT E } \\
41,53\end{array}$ & $\begin{array}{l}\text { OREN } \\
11953\end{array}$ & $\begin{array}{l}\text { SAMPLEE } \\
10793\end{array}$ \\
\hline$y=232$ & 000 & 1961 & ODS & $02 / 08 / 65$ & 522.500 & 0.9859 & 7.65808 & 8.26 & 22,87 & 12848 & $1082 \mathrm{a}$ \\
\hline U. -232 & 000 & 1961 & ODS & $02 / 08 / 65$ & 523.500 & 0.9638 & 7.62885 & 21.45 & 59,24 & 11868 & 10597 \\
\hline$U=232$ & 000 & 1961 & ODS & $02 / 08 / 65$ & 524.500 & 0.9475 & 7.59970 & 31.35 & 86,42 & 12042 & 10571 \\
\hline$U=232$ & 000 & 1961 & ODS & $02 / 08 / 65$ & 525.500 & 0.9331 & 7.57089 & 40.26 & 110,79 & 11845 & 10240 \\
\hline$U=232$ & 000 & 1961 & ODS & $02 / 08 / 65$ & 526.500 & 0,9112 & 7.54216 & 54.04 & 148,41 & 12055 & 10177 \\
\hline$u=232$ & 000 & 1961 & ODS & $02 / 08 / 65$ & 527.500 & 0,9470 & 7,51350 & 31.65 & 86.76 & 11900 & 10440 \\
\hline U. 232 & 000 & 1961 & ODS & $02 / 08 / 65$ & 528.500 & 0,9353 & 7.48519 & 38.87 & 106,35 & 12130 & 10511 \\
\hline$U=232$ & 000 & 1961 & ODS & $02 / 08 / 65$ & 529.500 & 0,9720 & 7.45694 & 16.51 & 45.08 & 11776 & 10604 \\
\hline$U=232$ & 000 & & ODS & $02 / 08 / 65$ & 530.500 & 0,9644 & 7.42885 & 21.06 & 57.40 & 12064 & 10778 \\
\hline$U=232$ & 000 & 1961 & ODS & $02 / 08 / 65$ & 531.500 & 0.9607 & 7,40093 & 23.31 & 63,42 & 11738 & $1044 \varepsilon$ \\
\hline$U=232$ & 000 & 1961 & ODS & $02 / 08 / 65$ & 532.500 & 0.9706 & 7.37315 & 17.36 & 47.14 & 11739 & 10556 \\
\hline$y=232$ & 000 & 1961 & ODS & $02 / 08 / 65$ & 533.500 & 0.9597 & 7.34554 & 23.89 & 64,75 & 11626 & 10337 \\
\hline$U_{1}=232$ & 000 & 1961 & ODS & $02 / 08 / 65$ & 534,500 & 0,9613 & 7,31808 & 22,95 & 62,08 & 11580 & 10313 \\
\hline$u=232$ & 000 & 1961 & ODS & $02 / 08 / 65$ & 535.500 & $0: 9754$ & 7,29077 & 14.47 & $39 ; 07$ & 11534 & 10426 \\
\hline$U=232$ & 000 & 1961 & ODS & $02 / 08 / 65$ & 536.500 & 0.9598 & 7,26362 & 23.84 & 64,26 & 11728 & 10427 \\
\hline$U=232$ & 000 & 1961 & ODS & $02 / 08 / 65$ & 537.500 & 0.9354 & 7,23662 & 38,84 & 104.49 & 11645 & 100 Bह \\
\hline$U=232$ & 000 & 1961 & ODS & $02 / 08 / 65$ & 538.500 & 0.9621 & 7.20977 & 22.49 & 60,38 & 11517 & 10260 \\
\hline$y=232$ & 000 & 1961 & ODS & $02 / 08 / 65$ & 539.500 & 0,9661 & 7,18306 & 20.03 & 53.68 & 11349 & 10198 \\
\hline U. 232 & 000 & 1961 & ODS & $02 / 08 / 65$ & 540.500 & 0,9667 & 7.15651 & 19.70 & 52.69 & 11579 & 10361 \\
\hline$u=232$ & 000 & 1961 & ODS & $02 / 08 / 65$ & 541.500 & 0,9659 & 7.13010 & 20.16 & 53.83 & 11361 & 10156 \\
\hline$U=232$ & 000 & 1961 & ODS & $02 / 08 / 65$ & 542.500 & 0.9589 & 7.10384 & 24.40 & 65,04 & 11334 & 10055 \\
\hline$y=232$ & 000 & 1961 & ODS & $02 / 08 / 65$ & 544.500 & 0.9585 & 7.05175 & 24.64 & 65,42 & 34172 & 30283 \\
\hline$V=232$ & 000 & 1961 & ODS & $02 / 08 / 65$ & 548.000 & 0,9448 & 0.96196 & 33.02 & 87.12 & 45184 & 39403 \\
\hline$y=232$ & 000 & 1961 & ODS & $02 / 08 / 65$ & 552.000 & 0,9454 & 6.86143 & 32.66 & 85.55 & 44431 & 38654 \\
\hline U. 232 & 000 & 1961 & ODS & $02 / 08 / 65$ & 556.500 & 0,9378 & 6,75091 & 37,33 & 96,99 & 54395 & 46881 \\
\hline$U=232$ & 000 & 1961 & ODS & $02 / 08 / 65$ & 561.000 & 0.9326 & 6.64304 & 40.56 & 104.53 & 43098 & 37032 \\
\hline$v=232$ & 000 & 1961 & ODS & $02 / 08 / 65$ & 565.000 & 0.9119 & 6.54931 & 53.62 & 137.22 & 42550 & 35807 \\
\hline$U=232$ & 000 & 1961 & ODS & $02 / 08 / 65$ & 569.000 & 0.8940 & 6.45755 & 65.11 & 165,45 & 41763 & 34498 \\
\hline$v=232$ & 000 & & ODS & $02 / 08 / 65$ & 571.500 & 0.8732 & 6.40118 & 78.81 & 199.40 & 10438 & 8427 \\
\hline$y=232$ & 000 & 1961 & ODS & $02 / 08 / 65$ & 572.500 & 0.8741 & 0.37886 & 78.25 & 197.63 & 10301 & 8326 \\
\hline $\begin{array}{l}y=232 \\
y=232\end{array}$ & 000 & 1961 & ODS & $02 / 08 / 65$ & 573.500 & 0.8377 & 0.35661 & 102.93 & 259.51 & 10223 & 79 \\
\hline $\begin{array}{l}u=232 \\
U=232\end{array}$ & 000 & 1961 & ODS & $02 / 08 / 65$ & 574.500 & 0.8443 & 6.33450 & 98.40 & 247.65 & 10432 & 8147 \\
\hline $\begin{array}{l}U=232 \\
U=232\end{array}$ & 000 & 1961 & ODS & $02 / 08 / 65$ & 575.500 & 0.8010 & 6.31251 & 124.62 & 313.10 & 10287 & 7680 \\
\hline $\begin{array}{l}=232 \\
U=232\end{array}$ & $\begin{array}{l}000 \\
000\end{array}$ & $\begin{array}{l}1961 \\
1961\end{array}$ & ODS & $\begin{array}{l}02 / 08 / 65 \\
02 / 08 / 65\end{array}$ & $\begin{array}{l}576.500 \\
577.500\end{array}$ & $\begin{array}{l}0.7965 \\
0.7454\end{array}$ & $\begin{array}{l}6.29063 \\
6.26886\end{array}$ & 132.28 & $\begin{array}{l}331.77 \\
427.59\end{array}$ & 10432 & $768 \varepsilon$ \\
\hline$u=232$ & 000 & 1961 & ODS & $02 / 08 / 65$ & 578.500 & 0,7292 & 0.24721 & $\begin{array}{l}170.78 \\
183.61\end{array}$ & $\begin{array}{l}427.59 \\
458,92\end{array}$ & $\begin{array}{l}10460 \\
10289\end{array}$ & $\begin{array}{l}7216 \\
6944\end{array}$ \\
\hline U. 232 & 000 & 1961 & ODS & $02 / 08 / 65$ & 579.500 & 0,6758 & 0.22566 & 227.83 & $568^{\circ}, 46$ & 10048 & 6285 \\
\hline$v=232$ & 000 & 1961 & ODS & $02 / 08 / 65$ & 580.500 & 0.6272 & 6.20423 & $271: 21$ & $675: 54$ & 10112 & 5871 \\
\hline$U=232$ & 000 & 1961 & ODS & $02 / 08 / 65$ & 581.500 & 0.5416 & 6.18291 & 356.42 & 886,25 & 10121 & 5075 \\
\hline U. 232 & 000 & 1961 & ODS & $02 / 08 / 65$ & 582.500 & 0,4757 & 0.16170 & 431.91 & 1072.12 & 9962 & 4387 \\
\hline$U=232$ & 000 & 1961 & ODS & $02 / 08 / 65$ & 583.500 & 0.3663 & 0.14060 & 583.83 & 1446,75 & 9844 & 3338 \\
\hline U. 232 & 000 & 1961 & ODS & $02 / 08 / 65$ & 584.500 & 0.2443 & 6.11961 & 819.18 & 2026.48 & 10033 & 2269 \\
\hline U. 232 & 000 & 1961 & ODS & $02 / 08 / 65$ & 585.500 & 0,1525 & 6.09872 & 1093,20 & $2699^{\circ} .72$ & 9862 & 1396 \\
\hline U. 232 & 000 & 1961 & ODS & $02 / 08 / 65$ & 586.500 & 0,0886 & 0.07794 & 1408,88 & $3473: 37$ & 9779 & 806 \\
\hline v. 232 & 000 & 1981 & ODS & $02 / 08 / 65$ & 587.500 & 0.0482 & 6.05727 & $2763.2^{8}$ & $433^{9} .71$ & 10001 & 445 \\
\hline U. 232 & 000 & 1961 & ODS & $02 / 08 / 65$ & 588.500 & 0.0299 & 6.03670 & 2040.90 & 5014.42 & 9798 & 270 \\
\hline U. 232 & 000 & 1961 & ODS & $02 / 08 / 65$ & 589.500 & 0.0227 & 6.01624 & $2200 \cdot 16$ & 5396.55 & $99_{17}$ & $20^{\varepsilon}$ \\
\hline U. 232 & 000 & 1961 & ODS & $02 / 08 / 65$ & 590.500 & 0.0187 & 5,99588 & 2313.09 & 5663,94 & 9680 & 167 \\
\hline U. 232 & 000 & 1961 & ODS & $02 / 08 / 65$ & 591.500 & 0,0182 & 5,97562 & 2327.52 & 5689.65 & 9641 & $16 \bar{c}$ \\
\hline $\begin{array}{l}U=232 \\
U=232\end{array}$ & 000 & 1961 & ODS & $02 / 08 / 65$ & 592.500 & 0.0152 & 5.95547 & 2432.36 & 5935.89 & 9765 & 137 \\
\hline & 000 & 1961 & ODS & $02 / 08 / 65$ & 593.500 & 0.0168 & 5.93542 & 2383.88 & 5807.77 & 9767 & $\therefore$ \\
\hline $\begin{array}{l}U=232 \\
U . \\
U .\end{array} 232$ & 000 & 1961 & ODS & $02 / 08 / 65$ & 594.500 & 0,0265 & 5.91546 & 2110.69 & 5133.57 & 9650 & 236 \\
\hline $\begin{array}{l}U=232 \\
U=232 \\
U=\end{array}$ & $\begin{array}{l}000 \\
000\end{array}$ & $\begin{array}{l}1961 \\
1961\end{array}$ & ODS & $02 / 08 / 65$ & 595,500 & 0,0542 & 5.89561 & 1694.49 & 4114.37 & 9586 & 480 \\
\hline $\begin{array}{l}\text { ve } 232 \\
U=232\end{array}$ & $\begin{array}{l}000 \\
000\end{array}$ & $\begin{array}{l}1961 \\
1961\end{array}$ & ODS & $02 / 08 / 65$ & 596.500 & 0.1216 & 5.87586 & 1224.64 & 2968,55 & 9728 & 1095 \\
\hline $\begin{array}{l}u=232 \\
U=232\end{array}$ & $\begin{array}{l}000 \\
000\end{array}$ & $\begin{array}{l}1961 \\
1961\end{array}$ & ODS & $02 / 08 / 65$ & 597.500 & 0.2177 & 5.85621 & 886.22 & 2144.61 & 9367 & 1887 \\
\hline $\begin{array}{l}=232 \\
U=232\end{array}$ & $\begin{array}{l}000 \\
000\end{array}$ & & ODS & $02 / 08 / 65$ & 598.500 & 0.3351 & 5.83666 & 635.56 & 1535,45 & 9628 & 2986 \\
\hline $\begin{array}{l}=232 \\
U=232\end{array}$ & $\begin{array}{l}000 \\
000\end{array}$ & $\begin{array}{l}1961 \\
1961\end{array}$ & ODS & $02 / 08 / 65$ & 599.500 & 0.4295 & 5.81720 & 491.24 & 1184,81 & 9312 & $370 a$ \\
\hline U. 232 & 000 & $\begin{array}{l}1961 \\
1961\end{array}$ & ODS & $\begin{array}{l}02 / 08 / 65 \\
02 / 68 / 65\end{array}$ & $\begin{array}{l}600.500 \\
601.500\end{array}$ & $\begin{array}{l}0,5125 \\
0,5831\end{array}$ & $\begin{array}{l}5.79784 \\
5.77858\end{array}$ & 388.55 & $\begin{array}{l}935 \\
753\end{array}$ & $\begin{array}{l}9523 \\
9524\end{array}$ & $451 \varepsilon$ \\
\hline$U=232$ & 000 & 1961 & & & & 0.0001 & 3,71858 & 313.57 & 753,79 & 9524 & 5141 \\
\hline $11=232$ & 000 & $\begin{array}{l}1961 \\
1961\end{array}$ & $\begin{array}{l}\text { ODS } \\
\text { ODS }\end{array}$ & $\begin{array}{l}02 / 08 / 65 \\
02 / 08 / 65\end{array}$ & $\begin{array}{l}602.500 \\
603.500\end{array}$ & $\begin{array}{l}0,6288 \\
0,6841\end{array}$ & $\begin{array}{l}5.75942 \\
5.74035\end{array}$ & 269.66 & 647,15 & 9438 & 5495 \\
\hline 232 & 000 & 1961 & ODS & $02 / 08 / 65$ & 604.5 & $\begin{array}{l}0.0841 \\
0.6945\end{array}$ & $\begin{array}{l}5,74035 \\
5,72137\end{array}$ & 220.73 & 528,85 & 9451 & 5986 \\
\hline$U=232$ & 000 & 1961 & ODS & $02 / 08 / 65$ & 605.500 & 0.7598 & $\begin{array}{l}5,72137 \\
5,70249\end{array}$ & $\begin{array}{l}211.94 \\
159.72\end{array}$ & 506.95 & 9640 & 6195 \\
\hline$u=232$ & 000 & 1961 & ODS & $02 / 08 / 65$ & 606,500 & 0,7947 & 5.68370 & $\begin{array}{l}159.72 \\
133,58\end{array}$ & & $\begin{array}{l}9297 \\
9981\end{array}$ & \\
\hline U. 232 & 000 & 1961 & ODS & $02 / 08 / 65$ & 607,500 & 0.8055 & 5.66500 & $\begin{array}{l}135,7 \\
125,7\end{array}$ & 318,46 & $\begin{array}{l}9281 \\
9227\end{array}$ & $\begin{array}{l}6825 \\
6886\end{array}$ \\
\hline$U=232$ & 000 & 1961 & ODS & $02 / 08 / 65$ & 608.500 & 0,8065 & 5.64640 & 125,00 & 297,03 & 9485 & 70 \\
\hline U. 232 & 000 & 1961 & ODS & $02 / 08 / 65$ & 609.500 & 0.8147 & 5.62788 & 119.14 & 282,54 & 9572 & 722 \\
\hline U. 232 & 000 & 1961 & ODS & $02 / 08 / 65$ & 610.500 & 0.8401 & 5.60946 & 101.31 & $\begin{array}{l}202,04 \\
239,94\end{array}$ & 9189 & 7145 \\
\hline$V=232$ & 000 & 1961 & ODS & $02 / 08 / 65$ & 613.500 & 0.8841 & 5.55474 & 71.60 & 168,74 & 44987 & 36840 \\
\hline U. 232 & 000 & 1961 & ODS & $02 / 08 / 65$ & 619.000 & 0.9163 & 5.45646 & 50 & $11^{8}, 72$ & & 45375 \\
\hline U. 232 & 000 & 1961 & ODS & $02 / 08 / 65$ & 625.000 & 0.92 & .35220 & 45. & 105,77 & & 45 \\
\hline v. 232 & 000 & 1961 & ODS & $02 / 08 / 65$ & 631.000 & 9665 & .25090 & 31.97 & 73.25 & 50475 & $4424 \bar{\epsilon}$ \\
\hline U. 232 & 000 & 1961 & ODS & $02 / 0$ & 637.0 & 0 & 15 & 25 & & 50 & \\
\hline U. 232 & 000 & 1961 & ODS & $02 / 0$ & 646.5 & 0 & & 23 & & 8 & 94 \\
\hline$U=232$ & 000 & 1961 & ODS & $02 / 08 / 65$ & 660.000 & 0.9676 & 4.79960 & 19. & 41 & 107838 & 9659 \\
\hline U. 232 & 000 & 1961 & ODS & $02 / 08 / 65$ & 674.000 & 0.9711 & 4.60228 & 17.06 & 36,60 & 103485 & 93031 \\
\hline U. 232 & 000 & 1961 & ODS & $02 / 08 / 65$ & 689.000 & 0,9713 & 4.40407 & 16,92 & 35,50 & 111308 & $10011 E$ \\
\hline$U=232$ & 000 & 1961 & ODS & $02 / 08 / 65$ & 705.500 & 0.9705 & 4.20048 & $17:$ & $35: 63$ & 109935 & 98827 \\
\hline U. & 000 & 1961 & ODS & $02 / 08 / 65$ & 723.000 & 0.9691 & 3.99959 & 18,27 & 36.53 & 109358 & $98_{168}$ \\
\hline$U=232$ & 000 & 1961 & ODS & $02 / 08 / 65$ & 741.500 & 0.9740 & .80251 & 15.33 & 29,89 & 107879 & 97257 \\
\hline$U=232$ & 000 & 1961 & ODS & $02 / 08 / 65$ & 762.000 & 0.9740 & 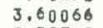 & 15.31 & 29,05 & 115381 & $10^{3}$ \\
\hline$U=232$ & 000 & $196 \hat{1}$ & ODS & $02 / 0$ & 784.500 & & 39 & 13 & & 106 & 95 \\
\hline U. 232 & 000 & 1961 & ODS & $02 / 08 / 65$ & 808.500 & 0.9660 & 3.19840 & 20 . & 35.94 & 99686 & $89_{1}$ \\
\hline$U=232$ & 000 & 1961 & ODS & $02 / 08 / 65$ & 835.000 & 0.9633 & 2.99861 & 21,74 & $37 ; 64$ & 89426 & 79793 \\
\hline$U=232$ & 000 & 1961 & ODS & $02 / 08 / 65$ & 864.500 & 0.9767 & 2.79745 & 13.70 & 22,91 & 73931 & \\
\hline$U=232$ & 000 & 1961 & ODS & $02 / 08 / 65$ & $\begin{array}{l}897.000 \\
933.500\end{array}$ & $\begin{array}{r}0.9757 \\
0.9625\end{array}$ & $\begin{array}{l}2.59841 \\
2.39919\end{array}$ & 14.29 & 23,04 & 53995 & $\begin{array}{l}4879 \varepsilon \\
2866 ?\end{array}$ \\
\hline 232 & 000 & 1961 & ODS & & & & & $22 \cdot 20$ & 4,39 & 32239 & \\
\hline
\end{tabular}




\begin{tabular}{|c|c|c|c|c|c|c|c|c|c|c|c|c|c|c|c|}
\hline \multicolumn{4}{|c|}{ IDENTIFICATION } & DATE & DELAY & TRANS, & ENERGY & $x=5 E C$ & $X$-SEC, & cour & & FRAC, & FAST & BKGS & No. PTS \\
\hline & 000 & 1962 & ODS & $1 / 08 / 65$ & 20,500 & 0,9796 & 4974.90540 & 11.5 & $\begin{array}{l}R 001 \mathrm{E} \\
816.20\end{array}$ & $\begin{array}{l}\text { OPEN } \\
99830\end{array}$ & $\begin{array}{l}\text { SAMPLE } \\
93192\end{array}$ & $\begin{array}{l}\text { OF OCT } \\
0.0170\end{array}$ & $\begin{array}{l}\text { OPEN } \\
20413\end{array}$ & $\begin{array}{l}\text { SAMPLE } \\
20209\end{array}$ & AVE. \\
\hline & 000 & 1962 & & & 23,000 & 0.9630 & 3952.18146 & 21.15 & 1329,90 & 72216 & 66270 & & 7958 & 7878 & 4 \\
\hline$=232$ & 000 & 1962 & ODS & $02 / 08 / 65$. & 24,500 & 0.9543 & 3483.05539 & 26.20 & $1546 \cdot 19$ & 37680 & 34267 & 0.0203 & 4085 & 4045 & 2 \\
\hline 232 & 000 & 1962 & ODS & $02 / 08 / 65$ & 25.500 & 0.9550 & 3215.23105 & 25.79 & 1462,32 & 36984 & 33659 & 0,0212 & 3815 & 3777 & 2 \\
\hline $\begin{array}{r}232 \\
232\end{array}$ & 000 & 1962 & 005 & $\begin{array}{l}02 / 08 / 65 \\
02 / 108 / 65\end{array}$ & $\begin{array}{l}26.500 \\
27.500\end{array}$ & $\begin{array}{l}0.9633 \\
0.9551\end{array}$ & $\begin{array}{l}2977,15057 \\
2764,56726\end{array}$ & $\begin{array}{l}20.98 \\
25,73\end{array}$ & $\begin{array}{l}1144.67 \\
1352,70\end{array}$ & $\begin{array}{l}35148 \\
32027\end{array}$ & $\begin{array}{l}32263 \\
29150\end{array}$ & $\begin{array}{l}0,0220 \\
0,0228\end{array}$ & $\begin{array}{l}3787 \\
3462\end{array}$ & $\begin{array}{l}3749 \\
3427\end{array}$ & $\frac{2}{2}$ \\
\hline $\begin{array}{l}= \\
u=232\end{array}$ & 000 & $\begin{array}{l}1962 \\
1962\end{array}$ & ODS & $02 / 08 / 65$ & $\begin{array}{l}27,500 \\
28,500\end{array}$ & $\begin{array}{l}0.9561 \\
0.9591\end{array}$ & 2573,96613 & 23,39 & 1186.69 & 29151 & 26644 & 0,0237 & 3896 & 3857 & 2 \\
\hline $\begin{array}{l}=.232 \\
U .232\end{array}$ & 000 & $\begin{array}{l}1962 \\
1962\end{array}$ & ODS & $02 / 08 / 65$ & $\begin{array}{l}28,200 \\
29.500\end{array}$ & 0,9385 & 2402,41769 & 35,55 & 1742,69 & 30650 & 27412 & 0,0245 & 2847 & 2818 & 2 \\
\hline 232 & 000 & & ODS & $02 / 08 / 65$ & 30,500 & 0.9665 & 2247,46463 & 19.08 & 904.61 & 32641 & 30063 & 0.0253 & 3121 & 3090 & 2 \\
\hline U. 232 & 000 & 1962 & $0 \mathrm{DS}$ & $02 / 08 / 65$ & 31,500 & 0.9648 & 2107,03351 & 20.10 & 922.82 & 35189 & 32350 & 0.0261 & 2706 & 2679 & \\
\hline $\begin{array}{l}u=232 \\
4=233\end{array}$ & 000 & 1962 & ODS & $02 / 08 / 65$ & 32.500 & 0.9643 & 1979,36473 & 20,37 & 906.20 & 38507 & 35383 & 0.0270 & 1982 & 1962 & 2 \\
\hline $\begin{array}{l}1-232 \\
-232\end{array}$ & 000 & $\begin{array}{l}1962 \\
1962\end{array}$ & $\begin{array}{l}\text { ODS } \\
\text { ODS }\end{array}$ & $\begin{array}{l}02 / 08 / 65 \\
02 / 08 / 65\end{array}$ & $\begin{array}{l}33,500 \\
34,500\end{array}$ & $\begin{array}{l}0.9509 \\
0.9753\end{array}$ & $\begin{array}{l}1862,95744 \\
1756,52509\end{array}$ & $\begin{array}{l}28,21 \\
14,03\end{array}$ & $\begin{array}{r}1217.59 \\
587,87\end{array}$ & $\begin{array}{l}38274 \\
38327\end{array}$ & $\begin{array}{l}34680 \\
35618\end{array}$ & $\begin{array}{l}0,0278 \\
0,0286\end{array}$ & $\begin{array}{l}2225 \\
2079\end{array}$ & $\begin{array}{l}2203 \\
2058\end{array}$ & 2 \\
\hline 232 & 000 & 1962 & ODS & $02 / 08 / 65$ & 5.500 & 0,9531 & 1658,95972 & 26.95 & 1097,70 & 88640 & 35091 & 0,0295 & 1855 & 1836 & \\
\hline 232 & 000 & 1962 & ODS & $02 / 08 / 65$ & 5.500 & 0,9596 & 1569,30305 & 3,11 & 915.66 & 7941 & 34691 & 0303 & 2470 & 2446 & \\
\hline $23 ?$ & 000 & $196 ?$ & ODS & & 50 & 9686 & 1486,72284 & 8.86 & 688,83 & 37073 & 34217 & 0,0311 & 2780 & 2752 & \\
\hline 232 & 000 & 1962 & ODS & $02 / 08 / 65$ & 38,500 & 0.9609 & 1410,49350 & 22.35 & 839.54 & 38246 & 35018 & 0.0320 & 2565 & 2539 & \\
\hline U. 232 & 000 & 1962 & oos & $02 / 08 / 65$ & 39,500 & 0.9571 & 1339,98012 & 24,60 & 900.56 & 37359 & 34068 & 0.0328 & 3298 & 3265 & \\
\hline U. 232 & 000 & 1962 & ODS & 021081 & 40.500 & 0.9564 & 1274.62520 & $\begin{array}{l}24.97 \\
25.74\end{array}$ & 891.65 & $\begin{array}{l}36954 \\
34509\end{array}$ & 33677 & 0336 & $\begin{array}{l}3344 \\
3869\end{array}$ & 3311 & \\
\hline & 000 & 1962 & ODS & 021 & 41,500 & 0.9551 & 121 & 25.74 & 896.85 & 34529 & 31423 & 0.0344 & 3869 & 3830 & \\
\hline$U=232$ & 000 & 1962 & ODS & $02 / 08 / 65$ & 42.500 & 0.9513 & 1157,48317 & 28.00 & 952.64 & 31230 & 28307 & 0.0353 & 4780 & 4732 & 2 \\
\hline $\begin{array}{l}232 \\
232\end{array}$ & $\begin{array}{l}000 \\
000\end{array}$ & $\begin{array}{l}1962 \\
1962\end{array}$ & $\begin{array}{l}\text { ODS } \\
\text { ODS }\end{array}$ & $\begin{array}{l}02 / 08 / 65 \\
02 / 08 / 65\end{array}$ & $\begin{array}{l}43,500 \\
44,500\end{array}$ & $\begin{array}{l}0.9441 \\
0.9417\end{array}$ & $\begin{array}{l}1104,87726 \\
1055,77780\end{array}$ & $\begin{array}{l}32.25 \\
33.66\end{array}$ & $\begin{array}{l}1071.88 \\
1093.66\end{array}$ & $\begin{array}{l}32354 \\
35042\end{array}$ & $\begin{array}{l}29104 \\
31442\end{array}$ & $\begin{array}{l}0,0361 \\
0,0369\end{array}$ & $\begin{array}{l}\begin{array}{l}4857 \\
4968\end{array} \\
\end{array}$ & $\begin{array}{l}4808 \\
4918\end{array}$ & 2 \\
\hline 232 & 000 & $\begin{array}{l}1906 \\
1962\end{array}$ & ODS & $02 / 0$ & 45,500 & 0.9573 & 1009,87996 & 24,48 & 777,97 & 35314 & 32209 & 0,0378 & 4937 & 4888 & \\
\hline$U=232$ & 000 & 1962 & ODS & $02 / 08 / 65$ & 46,500 & 0.9566 & 966,91132 & 24,84 & 772.42 & 35881 & 32705 & 0.0386 & 5075 & 5024 & \\
\hline$U=232$ & 000 & 1962 & ODS & 02108 & 47.500 & 0.9619 & 926.62781 & 21.78 & 662.90 & 34900 & 31985 & 0.0394 & 5858 & 5800 & \\
\hline $\begin{array}{l}y=232 \\
y=232\end{array}$ & 000 & 1962 & $\begin{array}{l}\text { ODS } \\
\text { ODS }\end{array}$ & $\begin{array}{l}02 / 08 / 65 \\
02 / 08 / 65\end{array}$ & $\begin{array}{l}48,500 \\
49,500\end{array}$ & 0,9480 & 888,81029 & 29,91 & 891.71 & $\begin{array}{l}36186 \\
34875\end{array}$ & $\begin{array}{l}32685 \\
32386\end{array}$ & 0.0403 & $\begin{array}{l}5487 \\
5710\end{array}$ & 5432 & \\
\hline $\begin{array}{l}y=232 \\
u=232\end{array}$ & 000 & $\begin{array}{l}1962 \\
1962\end{array}$ & $\begin{array}{l}005 \\
0015\end{array}$ & $\begin{array}{l}02 / 08 / 65 \\
02\end{array}$ & $\begin{array}{l}49.500 \\
50.500\end{array}$ & $\begin{array}{l}0.9747 \\
0.9613\end{array}$ & $\begin{array}{l}853,26 \\
810^{2} 88\end{array}$ & $\begin{array}{l}14.39 \\
23.11\end{array}$ & $\begin{array}{l}420.32 \\
6.32,93\end{array}$ & $\begin{array}{l}34875 \\
35178\end{array}$ & $\begin{array}{l}32386 \\
32230\end{array}$ & $\begin{array}{l}0.0411 \\
0.0419\end{array}$ & $\begin{array}{l}7119 \\
5402\end{array}$ & 5348 & 2 \\
\hline $\begin{array}{l}0.232 \\
u=232\end{array}$ & $\begin{array}{l}0.00 \\
000\end{array}$ & $\begin{array}{l}1962 \\
1962\end{array}$ & ODS & $02 / 08 / 65$ & $\begin{array}{l}50,500 \\
51.500\end{array}$ & $\begin{array}{l}0,9613 \\
0.9246\end{array}$ & $\begin{array}{l}819,80354 \\
788,27561\end{array}$ & $\begin{array}{l}22.11 \\
43.94\end{array}$ & $\begin{array}{r}6.32,93 \\
1233,58\end{array}$ & $\begin{array}{l}35178 \\
35691\end{array}$ & $\begin{array}{l}32220 \\
31441\end{array}$ & 0,0427 & 5295 & 5242 & 2 \\
\hline$u=232$ & 000 & 1962 & ODS & $02 / 08 / 65$ & 52.500 & 0.9392 & 758,53206 & 35.14 & 967.80 & 35426 & 31701 & 0.0436 & 5158 & 5107 & \\
\hline & 000 & 1962 & & & 500 & 9427 & & & & 34593 & 31068 & 0.0444 & 5255 & 5202 & \\
\hline & 000 & 1962 & ODS & & 4,500 & .9536 & 8148 & 26.64 & 706.77 & 34698 & 31524 & 0.0452 & 5110 & 5059 & \\
\hline 232 & 000 & 1962 & ODS & 021 & 55,500 & 0,9392 & 678 & 35.15 & 915.65 & $\begin{array}{l}35030 \\
34407\end{array}$ & 31345 & 0.0461 & $\begin{array}{l}4438 \\
4750\end{array}$ & 4394 & \\
\hline 232 & 000 & 1962 & ODS & $02 / 08 / 65$ & 56.500 & 0.9465 & 654,93116 & 30.82 & 788.75 & $\begin{array}{l}34407 \\
34077\end{array}$ & 31026 & 0.0469 & $\begin{array}{l}4759 \\
4025\end{array}$ & 471 & \\
\hline $\begin{array}{l}232 \\
233\end{array}$ & 000 & $\begin{array}{l}1962 \\
1962\end{array}$ & $\begin{array}{l}\text { ODS } \\
\text { ODS }\end{array}$ & $\begin{array}{l}02 / 0 \\
02210\end{array}$ & $\begin{array}{l}57.500 \\
58.500\end{array}$ & $\begin{array}{l}0.9301 \\
0.9451\end{array}$ & $\begin{array}{l}632,34903 \\
610,91504\end{array}$ & $\begin{array}{l}40.60 \\
31.64\end{array}$ & $\begin{array}{r}10<0.000 \\
781.92\end{array}$ & 34023 & $\begin{array}{l}30574 \\
30635\end{array}$ & 0,0486 & $\begin{array}{l}4025 \\
3904\end{array}$ & $\begin{array}{l}38864 \\
3865\end{array}$ & $\begin{array}{l}2 \\
2\end{array}$ \\
\hline 232 & $\begin{array}{l}000 \\
000\end{array}$ & $\begin{array}{l}1962 \\
1962\end{array}$ & ODS & $02 / 08 / 65$ & $\begin{array}{l}38,500 \\
59,500\end{array}$ & $\begin{array}{l}0.9431 \\
0.9303\end{array}$ & 590,55264 & $\begin{array}{l}31,04 \\
40,50\end{array}$ & 984,25 & 33660 & 29832 & 0,0494 & 3378 & 3344 & 2 \\
\hline & 000 & 1962 & ODS & $02 / 0$ & 60.500 & 0,9342 & 571,19158 & & & 34267 & 30497 & 0,0502 & 3108 & 3077 & \\
\hline$v=232$ & 000 & 1962 & ODS & $02 / 08 / 65$ & 61.500 & 0.9340 & 552,76727 & 38,28 & 899.97 & 33538 & 29842 & 0,0510 & 2932 & 2903 & 2 \\
\hline U. 232 & 000 & 1962 & ODS & $02 / 08 / 6$ & 62,500 & 0,96 & 535. & 19.8 & 458.32 & 33526 & 30831 & 9 & 2623 & 96 & $5+2+$ \\
\hline & 000 & 1962 & ODS & & & 0 & & 1 & & & & 15 & $\begin{array}{l}2231 \\
2072\end{array}$ & $\begin{array}{r}2209 \\
2251\end{array}$ & \\
\hline $\begin{array}{l}232 \\
232\end{array}$ & 000 & 1962 & 005 & & $\begin{array}{l}4.500 \\
5.500\end{array}$ & $\begin{array}{l}.2929 \\
9385\end{array}$ & 487,31519 & $\begin{array}{l}4.57 \\
35.55\end{array}$ & $\begin{array}{l}994.01 \\
784.73\end{array}$ & $\begin{array}{l}34421 \\
33367\end{array}$ & 29834 & $\begin{array}{l}0.0535 \\
0.0544\end{array}$ & 2050 & 2029 & \\
\hline 232 & 000 & $\begin{array}{l}1902 \\
1962\end{array}$ & 促 & $02 / 0$ & 5.500 & 99495 & 47276929 & $?$ & 63 & & & 0.0552 & & 1555 & \\
\hline 232 & 000 & 1962 & ODS & $02 / 0$ & 67.500 & 0.9269 & 5507 & 42.57 & 911,81 & 32519 & 28714 & 0,0 & 1851 & 1833 & \\
\hline 232 & 000 & 1962 & ODS & & 8,500 & 0.9177 & 35 & 4 & 10 & 88 & & 9 & 1630 & 1614 & \\
\hline 232 & 000 & 1962 & ODS & 021 & 9.500 & 0.9387 & 432.83 & 35. $\mathrm{C}$ & 738.23 & 31038 & 27754 & 0,0 & 1516 & 1501 & \\
\hline-232 & 000 & 1962 & ODS & & 70,500 & 0,9409 & 420,64363 & 34.16 & 700.68 & 30733 & & & 1484 & 1469 & \\
\hline$v=232$ & 000 & 1962 & $0 D S$ & $02 / 081$ & 71.500 & 0.9374 & 408,95965 & 36.24 & 732,94 & 30233 & 26997 & 0.0593 & 1297 & 1284 & \\
\hline 232 & 000 & 1962 & ODS & $02 / 08 / 65$ & 72,500 & 0.9489 & 397,75581 & 29.40 & 586.34 & 29422 & 26596 & 0,0602 & 1299 & 1286 & \\
\hline & 000 & 1962 & & & & & & & 10 & 27 & 24484 & 0.0610 & 1233 & 1221 & \\
\hline$u=232$ & 000 & 1962 & ODS & 021 & 74,500 & 0.9 & 376 & & & & & & & 20 & \\
\hline $\begin{array}{l}232 \\
233\end{array}$ & 000 & 1962 & ODS & & 75. & & & & & & 17 & & $\begin{array}{l}1336 \\
1103\end{array}$ & 1323 & 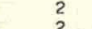 \\
\hline $\begin{array}{l}232 \\
232\end{array}$ & 000 & 1962 & ODS & 021 & $\begin{array}{l}76.500 \\
77.500\end{array}$ & $\begin{array}{l}0.9246 \\
0.9203\end{array}$ & $\begin{array}{l}357,24789 \\
348,08807\end{array}$ & $\begin{array}{l}43 \\
46\end{array}$ & $\begin{array}{l}830 \\
868\end{array}$ & $\begin{array}{r}13150 \\
8054\end{array}$ & $\begin{array}{r}11583 \\
7061\end{array}$ & $\begin{array}{l}0.0635 \\
0.0643\end{array}$ & $\begin{array}{l}1103 \\
1217\end{array}$ & $\begin{array}{l}1092 \\
1204\end{array}$ & 2 \\
\hline ( & 000 & $\begin{array}{l}1962 \\
1962\end{array}$ & ODS & 0210 & 78.500 & 0.9147 & 339,27607 & $\begin{array}{l}49.30 \\
49,96\end{array}$ & 920. & $\begin{array}{l}0054 \\
9308\end{array}$ & 8110 & 0,0652 & 1010 & 1000 & 2 \\
\hline ( & 000 & 1962 & ODS & & 79.5 & 0.9 & 330.79451 & 40. & 738 & 14646 & 12977 & 0.0660 & 1064 & 1054 & 2 \\
\hline 232 & 000 & 1962 & ODS & 021 & $80 \%$ & 0,9182 & 322,62 & 47. & 859.23 & 20808 & 18199 & 0.0668 & 1104 & 1093 & 2 \\
\hline u. 232 & 000 & 1962 & ons & $02 / 08 / 65$ & 81,500 & 0,9208 & 314.75840 & 46.26 & 820.72 & 24469 & 21462 & 0.0676 & 1199 & 1187 & \\
\hline$u=232$ & 000 & 1962 & ODS & $02 / 08 / 65$ & .500 & .93 & 307.17414 & 35,27 & 61 & 2703 & 241 & 0,06 & 1258 & 12 & 2 \\
\hline $\begin{array}{l}232 \\
232\end{array}$ & 000 & 1962 & ODS & $02 / 08 / 65$ & 83.500 & & & & & & & & 13 & 13321 & 2 \\
\hline 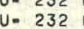 & 000 & 1962 & ODS & $\begin{array}{l}02 / 0 \\
02 / 0\end{array}$ & $\begin{array}{l}84, \\
85,\end{array}$ & & & & & & & & & & \\
\hline 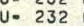 & 000 & 1962 & ODS & 021 & 86,500 & 0,9505 & 279,421 & 28,47 & 475.94 & 30411 & 27532 & 0.0718 & 1445 & 1430 & 2 \\
\hline 23 & 000 & 1962 & ODS & & 87. & 0,9 & 27 & & & & & & 1424 & 1410 & \\
\hline 232 & 000 & 1962 & ODS & 021 & $8 \varepsilon-2-2$ & & & & & & & & & 1500 & \\
\hline 2 & 000 & 1902 & UOS & & 89, & 0,8 & & 95 & 154 & & & & & 1418 & \\
\hline & 000 & 196 & 00 & & & & 255 & & & & $\begin{array}{l}25483 \\
28147\end{array}$ & & 1345 & 1333 & \\
\hline & 500 & 1962 & & $02 / 08$ & 91 , & 0. & 249.71829 & 32 & 516.09 & 31324 & 28147 & & 1345 & 1332 & \\
\hline 232 & 000 & 1962 & ODS & $02 / 08 / 65$ & 92,500 & 0,9607 & 244,34817 & 22.49 & 351.60 & 31353 & 28688 & 0.0768 & 1661 & 1644 & \\
\hline 年 & $\begin{array}{l}000 \\
000\end{array}$ & $\begin{array}{l}1962 \\
1962\end{array}$ & s & $\begin{array}{l}02 / 08 / 65 \\
02 / 08 / 65\end{array}$ & $\begin{array}{l}93.500 \\
94.500\end{array}$ & $\begin{array}{l}0.9623 \\
0.9612\end{array}$ & $\begin{array}{l}239: 14942 \\
234 ; 11483\end{array}$ & $\begin{array}{l}21.55 \\
22,19\end{array}$ & $\begin{array}{l}333.20 \\
339: 50\end{array}$ & $\begin{array}{l}31341 \\
31314\end{array}$ & $\begin{array}{l}28726 \\
28668\end{array}$ & $\begin{array}{l}0,0776 \\
0.0784\end{array}$ & $\begin{array}{l}1424 \\
1586\end{array}$ & $\begin{array}{l}1410 \\
1571\end{array}$ & 2 \\
\hline 236 & 000 & 1962 & ODS & & 95,500 & 0.000 & & & & & & & & 1457 & \\
\hline & 000 & 1962 & ODS & 021 & 96,500 & 0,95 & & & & & & & & 1427 & 2 \\
\hline$=$ & 000 & 196 & ODS & & 97 & $0: 2$ & & & & & $\begin{array}{l}27 \\
28\end{array}$ & & $\frac{12}{13}$ & $\begin{array}{l}1283 \\
1368\end{array}$ & \\
\hline & 000 & 1962 & ODS & & & & & & & & & & & & \\
\hline $\begin{array}{l}y=232 \\
y=232\end{array}$ & $\begin{array}{l}000 \\
000\end{array}$ & $\begin{array}{l}1962 \\
1962\end{array}$ & $\begin{array}{l}\text { ODS } \\
\text { ODS }\end{array}$ & $\begin{array}{l}02 / 08 \\
02 / 08\end{array}$ & 10,500 & $\begin{array}{l}0.9 \\
0.98\end{array}$ & & & & & 28 & & 13 & 1360 & \\
\hline$u=232$ & 000 & 1962 & ODS & $02 / 08 / 65$ & 101.500 & 0.9590 & 202,93664 & 23.48 & 334,47 & 30621 & 27968 & 0.0842 & 129 & 1280 & 2 \\
\hline 232 & 000 & 1962 & ODS & 02 & 02.500 & 0,9541 & 198 & 26.3 & & 30721 & & & 1 & 1. & \\
\hline & & & & & & & & & & & & & & & \\
\hline & 000 & 196 & ODS & & & & & & 16.34 & & & & 12 & 1227 & \\
\hline & 00 & 196 & $\mathrm{~s}$ & & & & & & & & & & & & \\
\hline & 00 & 1962 & & & & & & & & & & & 7 & $\begin{array}{l}1150 \\
1156\end{array}$ & \\
\hline & 000 & 1962 & ODS & $02 / 0$ & 108 & 0.9 & 177 & 42 & & & & & & 1163 & \\
\hline & 000 & 1968 & $s$ & $02 / 0$ & 109 & 9 & & & & & & & 10 & $\begin{array}{l}1101 \\
1006\end{array}$ & \\
\hline & 000 & 196 & $\begin{array}{l}\text { DDS } \\
\text { DAS }\end{array}$ & $\begin{array}{l}02 / 08 / 65 \\
02 / 08 / 65\end{array}$ & $\begin{array}{l}.500 \\
500\end{array}$ & 9632 & $\begin{array}{l}171,22532 \\
168,16779\end{array}$ & $\begin{array}{l}26.53 \\
21.01\end{array}$ & $\begin{array}{l}347,14 \\
272,46\end{array}$ & $\begin{array}{l}30622 \\
30717\end{array}$ & $\begin{array}{l}277815 \\
28177\end{array}$ & $\begin{array}{l}0.0917 \\
0.0925\end{array}$ & $\begin{array}{l}1057 \\
1160\end{array}$ & $\begin{array}{l}1046 \\
1148\end{array}$ & \\
\hline
\end{tabular}




\begin{tabular}{|c|c|c|c|c|c|c|c|c|c|c|c|}
\hline IDENTIFICATION & DELAY & TRANS, & ENERGY & $x$-sec & $x$ X-SEC: & cou & ITS & FRAC, & FAST & GSTES & iv \\
\hline $\begin{array}{lllll}232 & 000 & 1962 & 005 & 02 / 08 / 65 \\
323 & 000 & 1962 & 005 & 0208 / 105\end{array}$ & 112.500 & 0.9505 & 165.19143 & 28,43 & 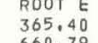 & $\begin{array}{c}\text { OPRN } \\
31381 \\
3381\end{array}$ & 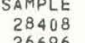 & $\begin{array}{l}\text { OF } \\
0.0934\end{array}-0934$ & $\begin{array}{c}O P E N \\
1034 \\
1034\end{array}$ & $\begin{array}{l}\text { AMMPE } \\
1024 \\
1024\end{array}$ & \\
\hline 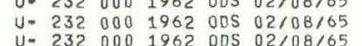 & $\begin{array}{l}3.500 \\
4.500\end{array}-1000$ & $\begin{array}{l}0.9116 \\
0.8329\end{array}-30$ & $\begin{array}{l}102,049359 \\
159,4095\end{array}$ & $\begin{array}{r}51,87 \\
102,47\end{array}$ & $\begin{array}{l}1293.95 \\
1295\end{array}$ & 30699 & 24352 & $\begin{array}{l}0.0942 \\
0.0950\end{array}-300$ & 902 & 893 & \\
\hline 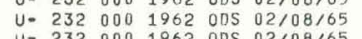 & 116,500 & $\begin{array}{l}0.918 \\
0.9175\end{array}$ & 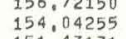 & $\begin{array}{l}72,522 \\
48,25\end{array}$ & $\begin{array}{l}908,85 \\
598,89\end{array}-198$ & $\begin{array}{l}3017 \\
3097 \\
3077\end{array}$ & $\begin{array}{l}2452 \\
2561 \\
25613\end{array}$ & $\begin{array}{l}0.0967 \\
0.0975\end{array}-37$ & 890 & 801 & \\
\hline 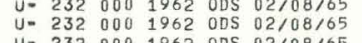 & $\begin{array}{l}117.500 \\
118,500 \\
500\end{array}$ & $\begin{array}{l}0,9070 \\
0,9305\end{array}$ & $\begin{array}{l}151 ; 317171 \\
148,88688\end{array}$ & $\begin{array}{l}54,30 \\
40,38\end{array}$ & $\begin{aligned} 673.18 \\
492.71\end{aligned}-120$ & 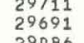 & $\begin{array}{l}25663 \\
26310 \\
23725\end{array}$ & $\begin{array}{l}0,0975 \\
0.0984\end{array}-0902$ & $\begin{array}{l}8914 \\
814\end{array}$ & $\begin{array}{l}928 \\
806 \\
820\end{array}$ & \\
\hline 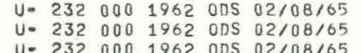 & $\begin{array}{l}119.500 \\
120.500 \\
121.500\end{array}$ & $\begin{array}{l}0.6497 \\
0.7558 \\
0.8794\end{array}$ & $\begin{array}{l}146,40528 \\
143 ; 98540 \\
141.62502\end{array}$ & $\begin{aligned} 91,27 \\
156,94 \\
72,01\end{aligned}$ & $\begin{array}{l}1104.36 \\
1838.14 \\
856.99\end{array}$ & $\begin{array}{l}29086 \\
29670 \\
28443\end{array}$ & $\begin{array}{l}23537 \\
21355 \\
23821\end{array}$ & $\begin{array}{l}0.0992 \\
0,1000 \\
0,1008\end{array}$ & $\begin{array}{l}795 \\
827 \\
924\end{array}$ & $\begin{array}{l}787 \\
819 \\
915\end{array}$ & \\
\hline 2320001962 ODS $02 / 08 / 65$ & 122.500 & 0.9539 & 139,32221 & 26.45 & 2,23 & 27119 & 24635 & 0.1017 & 963 & 53 & \\
\hline 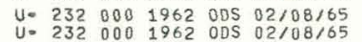 & $\begin{array}{l}123,500 \\
124500\end{array}$ & $\begin{array}{l}0: 9512 \\
0 ; 9319\end{array}$ & $\begin{array}{l}137 ; 07512 \\
134,88195\end{array}$ & $\begin{array}{l}28,03 \\
39,55\end{array}$ & $\begin{array}{l}328,19 \\
459,33\end{array}$ & $\begin{array}{l}25971 \\
25444\end{array}$ & $\begin{array}{l}23525 \\
21426\end{array}$ & $\begin{array}{l}0,1025 \\
0 ; 1033\end{array}$ & $\begin{array}{l}792 \\
848\end{array}$ & $\begin{array}{l}784 \\
840\end{array}$ & \\
\hline 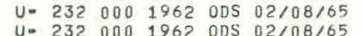 & $\begin{array}{l}125,500 \\
126500\end{array}$ & 0.8538 & $\begin{array}{l}132,74100 \\
130,65063\end{array}$ & $\begin{array}{r}88,60 \\
168.15\end{array}$ & $\begin{array}{l}1020,84 \\
1020.05\end{array}$ & $\begin{array}{r}23293 \\
2441\end{array}$ & & & 869 & $\begin{array}{l}860 \\
762\end{array}$ & \\
\hline $\begin{array}{lllll}232 & 000 & 1962 & 0 D S & 0 \\
232 & 000 & 1962 & \text { ODS } & 0\end{array}$ & $\begin{array}{l}126.500 \\
127,500\end{array}$ & (1) 0,8241 & $\begin{array}{l}{ }_{128,60924}^{130,6063} \\
1200\end{array}$ & $\begin{array}{l}\begin{array}{l}168,15 \\
108,46\end{array} \\
1\end{array}$ & $\begin{array}{l}1922,05 \\
1229,95\end{array}-\mathrm{V}$ & $\begin{array}{l}24441 \\
26179\end{array}$ & $\begin{array}{l}1524 \\
20544\end{array}$ & $\begin{array}{l}0.1050 \\
0.1058\end{array}$ & 755 & $\begin{array}{l}782 \\
747\end{array}$ & \\
\hline 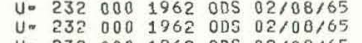 & $\begin{array}{l}128,500 \\
129,500\end{array}$ & $\begin{array}{l}0.8927 \\
0.9442\end{array}$ & $\begin{array}{l}226,61533 \\
124: 66743\end{array}$ & $\begin{array}{l}63.65 \\
32,19\end{array}$ & $\begin{array}{l}7166,19 \\
359,44\end{array}$ & $\begin{array}{l}28558 \\
28995\end{array}$ & $\begin{array}{l}2484 \\
26070\end{array}$ & $\begin{array}{l}0.1067 \\
0,1075\end{array}$ & $\begin{array}{l}689 \\
823\end{array}$ & $\begin{array}{l}682 \\
815\end{array}$ & \\
\hline 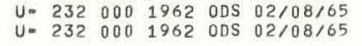 & $\begin{array}{l}130,500 \\
131,500\end{array}$ & $\begin{array}{l}0.9654 \\
0.9749\end{array}$ & $\begin{array}{l}122,76414 \\
120 ; 90410\end{array}$ & $\begin{array}{l}19,76 \\
14.23\end{array}$ & $\begin{array}{l}218,96 \\
156,50\end{array}$ & $\begin{array}{l}29453 \\
29160\end{array}$ & $\begin{array}{l}27775 \\
27072\end{array}$ & $\begin{array}{l}0.1083 \\
0,1091\end{array}$ & $\begin{array}{l}856 \\
914\end{array}-10$ & $\begin{array}{l}847 \\
905\end{array}$ & \\
\hline 0001962 ons $02 / 08 / 65$ & & 0.9505 & 119,08602 & 28,43 & 310,20 & 29882 & 148 & 0,1100 & 729 & 22 & \\
\hline 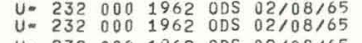 & $\begin{array}{l}1333,500 \\
134,500\end{array}$ & $\begin{array}{l}0: 9658 \\
0: 8813\end{array}$ & $\begin{array}{l}\frac{117}{115: 350864} \\
1157076\end{array}$ & $\begin{array}{l}19.48 \\
70: 81\end{array}$ & $\begin{array}{l}211,00 \\
761,19\end{array}$ & 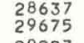 & $\begin{array}{l}26336 \\
24904\end{array}$ & $\begin{array}{l}0,1108 \\
0,1116\end{array}$ & $\begin{array}{l}780 \\
842\end{array}$ & $\begin{array}{l}773 \\
833\end{array}$ & \\
\hline $\begin{array}{lllll}232 & 000 & 1962 & 005 & 02 / 08 / 65 \\
232 & 000 & 1962 & 005 & 022 / 08 / 65\end{array}$ & $\begin{array}{l}135,500 \\
136,500\end{array}$ & $\begin{array}{l}0.8750 \\
0.9052\end{array}$ & $\begin{array}{l}113,87122 \\
112,20888\end{array}$ & $\begin{array}{l}74,86 \\
55,85\end{array}$ & $\begin{array}{l}798.84 \\
591.60\end{array}$ & $\begin{array}{l}29227 \\
29648\end{array}$ & $\begin{array}{l}24351 \\
25554\end{array}$ & $\begin{array}{l}0.1125 \\
0,1133\end{array}$ & $\begin{array}{l}871 \\
894\end{array}$ & $\begin{array}{l}862 \\
885\end{array}$ & \\
\hline 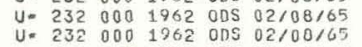 & $\begin{array}{l}137.500 \\
130 \\
1300\end{array}$ & $\begin{array}{l}0.8015 \\
0.051\end{array}$ & $\begin{array}{l}110,58269 \\
108,99159\end{array}$ & 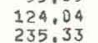 & $\begin{array}{l}1304.36 \\
2456.81\end{array}$ & $\begin{array}{l}28953 \\
2963\end{array}-1030$ & 96 & $\begin{array}{l}0.1141 \\
0 ; 1150 \\
0 ; 1150\end{array}$ & $\begin{array}{l}897 \\
780\end{array}$ & $\begin{array}{l}888 \\
772\end{array}$ & \\
\hline & $\begin{array}{l}13 \\
14\end{array}$ & $\begin{array}{l}0.7451 \\
0.8911\end{array}$ & $\begin{array}{l}107 ; 43459 \\
105,91072\end{array}$ & $\begin{array}{c}164,95 \\
64.65\end{array}$ & $\begin{array}{l}1709,73 \\
605,28\end{array}$ & $\begin{array}{l}29504 \\
29467\end{array}$ & & & $\begin{array}{l}792 \\
961\end{array}$ & $\begin{array}{l}784 \\
951\end{array}$ & \\
\hline U. 23200019620 & 141,500 & 0,8917 & 104,41903 & 64,25 & & & & & & & \\
\hline 2320001962 o0s $02 / 08 / 65$ & 142,500 & 0.9480 & 102,95864 & 29.9 & 303.57 & 29055 & 26228 & 1183 & 973 & 963 & \\
\hline 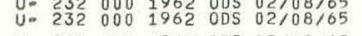 & $\begin{array}{l}143,500 \\
144,500\end{array}$ & $\begin{array}{l}0: 961 \frac{1}{0} \\
0,9823\end{array}$ & $\begin{array}{l}101.52868 \\
100,12830\end{array}$ & $\begin{array}{l}188 ; \\
10 ; 0\end{array}$ & $\begin{array}{l}188,91 \\
100,24\end{array}$ & $\begin{array}{l}29601 \\
29258 \\
258\end{array}$ & $\begin{array}{l}26761 \\
27365\end{array}$ & $\begin{array}{l}0: 1191 \\
0: 1199\end{array}$ & $\begin{array}{r}934 \\
1044\end{array}$ & $\begin{array}{r}944 \\
1034\end{array}$ & \\
\hline 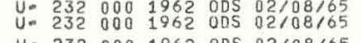 & $\begin{array}{l}1455: 500 \\
146: 500\end{array}$ & $\begin{array}{l}8: 9789 \\
0: 9668\end{array}$ & $\begin{array}{ll}98.56570 \\
97,41308\end{array}$ & $\begin{array}{l}11,93 \\
18,92\end{array}$ & $\begin{array}{l}1188,58 \\
186: 70\end{array}$ & $\begin{array}{l}29144 \\
29254\end{array}-100$ & $\begin{array}{l}27765 \\
26930\end{array}$ & $\begin{array}{l}0.1208 \\
0 ; 1216\end{array}$ & 929 & $\begin{array}{l}920 \\
895\end{array}$ & \\
\hline 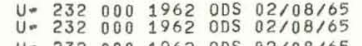 & $\begin{array}{l}147,500 \\
148,500\end{array}$ & $\begin{array}{l}0: 9819 \\
0: 9671\end{array}$ & $\begin{array}{ll}966: 09671 \\
94: 80693\end{array}$ & $\begin{array}{l}10.21 \\
18,73\end{array}$ & $\begin{array}{l}100013 \\
182 ; 38\end{array} \quad \longrightarrow$ & $\begin{array}{l}295056 \\
29322\end{array}$ & $\begin{array}{l}27166 \\
27001\end{array}$ & $\begin{array}{l}0.1224 \\
0: 1233\end{array}-1$ & $\begin{array}{l}928 \\
1036\end{array}$ & $\begin{array}{r}918 \\
1026\end{array}$ & \\
\hline 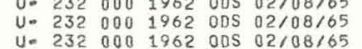 & $\begin{array}{l}150.500 \\
150 \\
151500\end{array}$ & $\begin{array}{l}0: 9744 \\
0.9842 \\
0.9842\end{array}$ & $\begin{array}{l}92,30379 \\
992 \\
990828\end{array}$ & $\begin{array}{r}9,77 \\
14.51 \\
8,92\end{array}$ & $\begin{aligned} 94,47 \\
139,43\end{aligned}$ & $\begin{array}{l}29805 \\
2902 \\
28033\end{array}$ & $\begin{array}{l}20692 \\
2092 \\
2713\end{array}$ & $\begin{array}{l}0.1249 \\
0.1249 \\
0.1257\end{array}$ & $\begin{array}{l}1993 \\
1053 \\
1032\end{array}$ & $\begin{array}{l}1648 \\
1042 \\
1022\end{array}$ & \\
\hline $005 \quad 02 / 08 / 65$ & 500 & 0.97 & 89,89859 & 3,3 & 126,94 & 405 & 336 & 1266 & 1141 & 1130 & \\
\hline $\begin{array}{l}O D S 02 / 08 / 65 \\
0 D S \\
021 / 8885\end{array}$ & $\begin{array}{l}153,500 \\
154,500\end{array}$ & 0.9 & $\begin{array}{l}88.73108 \\
87,58618\end{array}$ & 5.38 & $\begin{array}{l}1448,81 \\
1.83\end{array}$ & $\begin{array}{l}29372 \\
2965\end{array}$ & 27208 & 0.1274 & $\begin{array}{l}1156 \\
1190\end{array}-10$ & $\begin{array}{l}1144 \\
1187\end{array}$ & \\
\hline . & & & 6329 & & & & & & 1314 & 1301 & \\
\hline 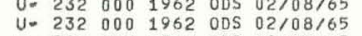 & $\begin{array}{l}156,500 \\
157,500\end{array}$ & $\begin{array}{l}0.97 \\
0: 97\end{array}$ & $\begin{array}{l}\begin{array}{l}85,36186 \\
84,28134\end{array} \\
\end{array}$ & $\begin{array}{l}13,69 \\
14,77\end{array}$ & $\begin{array}{l}122.52 \\
135.58\end{array}$ & $\begin{array}{l}2919 \\
28673\end{array}$ & $\begin{array}{l}27241 \\
26589\end{array}$ & $\begin{array}{l}0.1299 \\
0,1307\end{array}$ & $\begin{array}{l}13100 \\
1339\end{array}-$ & $\begin{array}{l}1297 \\
1326\end{array}$ & \\
\hline $\begin{array}{l}19620050202 / 18 / 65 \\
1962 \quad 005 \quad 02 / 08 / 65\end{array}$ & $\begin{array}{l}158,500 \\
159,500\end{array}$ & $\begin{array}{l}0.96 \\
0.97\end{array}-97-3$ & $\begin{array}{l}83,22121 \\
82,18095\end{array}$ & 14. & $\begin{array}{l}1990,83 \\
132,74\end{array}-\mathrm{l}-\mathrm{l}$ & $\begin{array}{l}288781 \\
28631\end{array}$ & & $\begin{array}{l}0.1316 \\
0.1324\end{array}$ & $\begin{array}{l}1371 \\
1399\end{array}-\mathrm{r}$ & $\begin{array}{l}1337 \\
1385\end{array}$ & \\
\hline 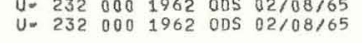 & $\begin{array}{l}1600.500 \\
161,500\end{array}$ & $\begin{array}{l}0.96 \\
0.96\end{array}$ & $\begin{array}{l}81.16008 \\
80.15811\end{array}$ & $\begin{array}{l}10.73 \\
21.82\end{array}$ & $\begin{array}{r}996,66 \\
195,34\end{array}$ & $\begin{array}{l}29119 \\
28851\end{array}$ & $\begin{array}{l}98 \\
19\end{array}$ & $\begin{array}{l}0.1332 \\
0.1340\end{array}$ & $\begin{array}{l}1473 \\
1406\end{array}$ & $\begin{array}{l}459 \\
451\end{array}$ & \\
\hline 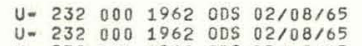 & 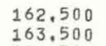 & $0:$ & $\begin{array}{l}17459 \\
0995\end{array}$ & & $\begin{array}{ll}32.09 \\
24.52\end{array}$ & $\begin{array}{l}720 \\
451\end{array}$ & & 49 & $\begin{array}{l}454 \\
579\end{array}$ & 39 & \\
\hline$\left\{\begin{array}{l}02 \\
022\end{array}\right.$ & & $\begin{array}{l}0.85 \\
0.65\end{array}$ & $\begin{array}{l}77.26107 \\
77.33023\end{array}$ & $\begin{array}{r}86.45 \\
238.26\end{array}$ & ${ }_{5}^{0}$ & $\begin{array}{l}28530 \\
2856 \\
2856\end{array}$ & & & $\begin{array}{l}1516 \\
1473\end{array}$ & $\begin{array}{l}1503 \\
158\end{array}$ & \\
\hline 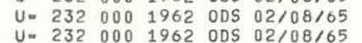 & $\begin{array}{l}166.500 \\
167500\end{array}$ & $\begin{array}{l}0.41 \\
0.53\end{array}$ & $\begin{array}{l}75,41610 \\
74,5130\end{array}$ & $\begin{array}{l}487 \\
349\end{array}$ & $\begin{array}{l}37.51 \\
237.91\end{array}$ & $\begin{array}{l}28154 \\
28192\end{array}$ & $\begin{array}{l}11223 \\
14384\end{array}$ & 82 & $\begin{array}{l}1595 \\
1594\end{array}$ & $\begin{array}{l}1579 \\
1578\end{array}$ & \\
\hline 1055 & $\begin{array}{l}168,500 \\
169,500\end{array}$ & $\begin{array}{l}0.801 \\
0.911\end{array}$ & 73 & $\begin{array}{r}123.98 \\
49.87\end{array}$ & $\begin{array}{r}1063,86 \\
45.442\end{array}$ & $\begin{array}{l}28312 \\
28299\end{array}$ & & $0:$ & $\begin{array}{l}1497 \\
1564\end{array}$ & $\begin{array}{l}1482 \\
1548\end{array}$ & \\
\hline 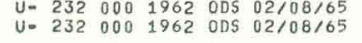 & $\begin{array}{l}170,500 \\
171.500\end{array}$ & $\begin{array}{l}0,9615 \\
0,9589\end{array}$ & $\begin{array}{l}71,91909 \\
71,08276\end{array}$ & $\begin{aligned} 21,98 \\
23,50\end{aligned}$ & $\begin{array}{l}196,39 \\
98,09\end{array}$ & $\begin{array}{l}28056 \\
27908\end{array}$ & $\begin{array}{l}25683 \\
25478\end{array}$ & $\begin{array}{l}15 \\
23 \\
23\end{array}$ & $\begin{array}{l}1594 \\
1500\end{array}$ & $\begin{array}{l}1558 \\
1544\end{array}$ & \\
\hline$U=2320001962$ ODS $02 / 08 / 65$ & 172.500 & 0.9536 & 70.26100 & 26.6 & 223.09 & 28251 & 25648 & 0,1432 & 1622 & 1605 & \\
\hline 65 & $\begin{array}{l}173 . \\
174 .\end{array}$ & $\begin{array}{l}0,97 \\
0.97\end{array}$ & $\begin{array}{l}69.45349 \\
68.65967\end{array}$ & 100 & & 21 & & & 1405 & & \\
\hline ODS $02 / 08 / 65$ & $\begin{array}{l}175.500 \\
176.500\end{array}$ & $0: 963$ & $6 \% .87945$ & & 173 & $\begin{array}{l}28 \\
28\end{array}$ & & & $\begin{array}{l}1402 \\
1391\end{array}$ & $\begin{array}{l}1388 \\
1377\end{array}$ & \\
\hline 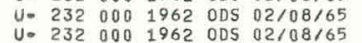 & $\begin{array}{l}177,500 \\
178,500\end{array}$ & $\begin{array}{l}0.9727 \\
0.9847\end{array}$ & $\begin{array}{l}66,35839 \\
65,61966\end{array}$ & $\begin{array}{c}15 \\
8 \\
\end{array}$ & $\begin{aligned} 126.24 \\
69.83\end{aligned}$ & $\begin{array}{l}28046 \\
27821\end{array}$ & & 3 & $\begin{array}{l}1360 \\
1317\end{array}$ & $\begin{array}{l}1347 \\
1304\end{array}$ & \\
\hline 65 & $\begin{array}{l}179,500 \\
180,500\end{array}$ & $\begin{array}{l}0.9690 \\
0,9560\end{array}$ & 64: & $\begin{array}{l}17 \\
25\end{array}$ & 201. & 27 & & & $\begin{array}{l}1264 \\
1332\end{array}$ & $\begin{array}{l}1252 \\
1318\end{array}$ & \\
\hline $232000 \quad 1962$ ODS $02 / 08 / 65$ & 181,500 & & 63,46573 & 21. & $5,1$. & 27734 & & & 1250 & 38 & \\
\hline 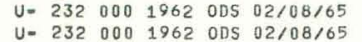 & $\begin{array}{r}182,500 \\
183500\end{array}$ & 0,99011 & 62,77212 & $\begin{array}{r}5.5 \\
13.0\end{array}$ & $\begin{array}{r}44,21 \\
103,11\end{array}$ & 516 & & & ${ }_{25}^{42}$ & 30 & \\
\hline $\begin{array}{l}021 \\
021 \\
021\end{array}$ & 10.0000 & 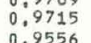 & $\begin{array}{l}61.41859 \\
60.7517\end{array}$ & 13.0 & $\begin{array}{ll}09 \\
51\end{array}$ & $\begin{array}{l}27806 \\
27806 \\
27611\end{array}$ & & & $\begin{array}{l}1220 \\
1220 \\
1189\end{array}$ & 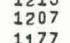 & \\
\hline 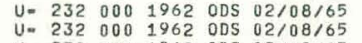 & $\begin{array}{l}185,500 \\
186.500\end{array}$ & $\begin{array}{l}0: 95 \\
0: 91\end{array}$ & $\begin{array}{l}60,75 \\
60,10\end{array}$ & $\begin{array}{l}25 \\
46\end{array}$ & $\begin{array}{l}\frac{1998,51}{363.02} \\
-102\end{array}$ & $\begin{array}{l}27711 \\
27528\end{array}$ & & & $\begin{array}{l}1189 \\
1242\end{array}$ & $\begin{array}{l}1177 \\
1229\end{array}$ & \\
\hline 1962 ODS $02 / 08 / 65$ & $\begin{array}{l}187,500 \\
188,500\end{array}$ & $\begin{array}{l}0.93 \\
0,95\end{array}$ & $\begin{array}{ll} & \end{array}$ & & 18 & & & & $\begin{array}{l}2206 \\
1130\end{array}$ & $\begin{array}{l}94 \\
19\end{array}$ & \\
\hline $\begin{array}{l}0.1962 \text { ODS } 02 / 108 / 65 \\
01962 \\
0\end{array}$ & $\begin{array}{l}190,500 \\
191500\end{array}$ & $\begin{array}{l}0.9619 \\
0.9863\end{array}$ & $\begin{array}{l}57.61063 \\
57.61053\end{array}$ & & 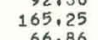 & 27613 & & & 1173 & 1011 & \\
\hline 5021 & & & & & & & & & 1161 & & \\
\hline $\begin{array}{l}00502 / 08 / 15 \\
005 \\
002 / 108 / 65\end{array}$ & 19 & & $55:$ & & & & & & 11 & & \\
\hline & & & & & & & & & & & \\
\hline & & & & & & & & & & & \\
\hline & & & & & & & & & & & \\
\hline p & $\begin{array}{l}199,500 \\
200,500\end{array}$ & $\begin{array}{l}0,8 \\
0.95\end{array}$ & & 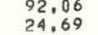 & & & & & & & \\
\hline 00019620 & & 0,9722 & 51,49 & & & & & & & & \\
\hline 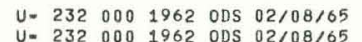 & 202,500 & 0.9739 & 50,98501 & 14,80 & & 26890 & 24 & & 1049 & 1039 & \\
\hline & & & & & & & & & & & \\
\hline & & & 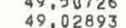 & & & & & & & & \\
\hline 1963 ons no? & & & & & & & & & 11 & & \\
\hline 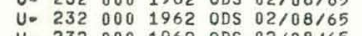 & & & 7.6 & & & & & & & & \\
\hline 19 & & & 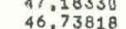 & & & & & & $\begin{array}{l}8977 \\
867\end{array}$ & & \\
\hline
\end{tabular}




\begin{tabular}{|c|c|c|}
\hline & IDEN & TIFIO \\
\hline U. & $\begin{array}{l}232 \\
232\end{array}$ & 000 \\
\hline & $\begin{array}{l}232 \\
232\end{array}$ & 000 \\
\hline U. & 232 & 000 \\
\hline u. & 232 & 000 \\
\hline U. & 232 & 000 \\
\hline U. & 232 & 000 \\
\hline U. & 232 & 000 \\
\hline U- & 232 & 000 \\
\hline U. & 232 & 000 \\
\hline U. & 232 & 000 \\
\hline$U=$ & 232 & 000 \\
\hline $\begin{array}{l}u= \\
u=\end{array}$ & $\begin{array}{l}232 \\
232\end{array}$ & $\begin{array}{l}000 \\
000\end{array}$ \\
\hline U. & 232 & 000 \\
\hline v. & 232 & 000 \\
\hline U. & 232 & 000 \\
\hline U. & 232 & 000 \\
\hline$U=$ & 232 & 000 \\
\hline$y=$ & 232 & 000 \\
\hline U. & 232 & 000 \\
\hline$U=$ & 232 & 000 \\
\hline U. & 232 & 000 \\
\hline U. & 232 & 000 \\
\hline$U=$ & 232 & 000 \\
\hline $\begin{array}{l}U= \\
U=\end{array}$ & $\begin{array}{l}232 \\
232\end{array}$ & 000 \\
\hline U. & $\begin{array}{l}232 \\
232\end{array}$ & $\begin{array}{l}000 \\
000\end{array}$ \\
\hline v. & $\begin{array}{l}232 \\
232\end{array}$ & 000 \\
\hline U. & 232 & 000 \\
\hline U. & 232 & 000 \\
\hline$u=$ & 232 & 000 \\
\hline U. & 232 & 000 \\
\hline U. & 232 & 000 \\
\hline$U=$ & 232 & 000 \\
\hline $\begin{array}{l}\text { U. } \\
u-\end{array}$ & $\begin{array}{l}232 \\
232\end{array}$ & 000 \\
\hline $\begin{array}{l}\text { U. } \\
\text { u. }\end{array}$ & $\begin{array}{l}232 \\
232\end{array}$ & 000 \\
\hline u. & $\begin{array}{l}232 \\
232\end{array}$ & 000 \\
\hline u. & $\begin{array}{l}232 \\
232\end{array}$ & $\begin{array}{l}000 \\
000\end{array}$ \\
\hline U. & 232 & 000 \\
\hline$u=$ & 232 & 000 \\
\hline U. & 232 & 000 \\
\hline U. & 232 & 000 \\
\hline$U=$ & 232 & 000 \\
\hline u- & 232 & 000 \\
\hline U. & 232 & 000 \\
\hline$u=$ & 232 & 000 \\
\hline u. & 232 & 000 \\
\hline v. & 232 & 000 \\
\hline u. & 232 & 000 \\
\hline U. & 232 & 000 \\
\hline U. & 232 & 000 \\
\hline U. & 232 & 000 \\
\hline U. & 232 & 000 \\
\hline U. & 232 & 000 \\
\hline U. & 232 & 000 \\
\hline U. & 232 & 000 \\
\hline U. & 232 & UUU \\
\hline U. & 232 & 000 \\
\hline U. & 232 & 000 \\
\hline U. & 232 & 000 \\
\hline u. & 232 & 000 \\
\hline U. & 232 & 000 \\
\hline U. & 232 & 000 \\
\hline U. & 232 & 000 \\
\hline$U=$ & 232 & 000 \\
\hline U. & 232 & 000 \\
\hline U. & 232 & 000 \\
\hline U. & 232 & 000 \\
\hline u= & 232 & 000 \\
\hline u- & 232 & 000 \\
\hline U: & $\begin{array}{l}232 \\
232\end{array}$ & $\begin{array}{ll}0 & 00 \\
0 & 0\end{array}$ \\
\hline U. & 232 & 000 \\
\hline u. & 232 & 000 \\
\hline u. & $\begin{array}{r}232 \\
233\end{array}$ & 000 \\
\hline & 232 & 000 \\
\hline$v=$ & $\begin{array}{l}232 \\
232\end{array}$ & 000 \\
\hline & $\begin{array}{l}232 \\
232\end{array}$ & 000 \\
\hline & & 000 \\
\hline J- & 232 & 000 \\
\hline j: & $\begin{array}{l}232 \\
232\end{array}$ & $\begin{array}{lll}0 & 0 & 0 \\
0 & 0 & 0\end{array}$ \\
\hline$U=$ & 232 & 000 \\
\hline U. & 232 & 000 \\
\hline U. & 232 & 000 \\
\hline U- & 232 & 000 \\
\hline 11. & 232 & 000 \\
\hline$U=$ & 232 & 000 \\
\hline$U=$ & 232 & 000 \\
\hline u. & 232 & 000 \\
\hline J. & & \\
\hline - & 232 & 000 \\
\hline U* & 232 & 000 \\
\hline 1. & 232 & 000 \\
\hline $1=$ & 232 & 000 \\
\hline 10 & 232 & 000 \\
\hline J:- & 232 & 000 \\
\hline : & 232 & 000 \\
\hline J & 232 & 000 \\
\hline
\end{tabular}




\begin{tabular}{|c|c|c|c|c|c|c|c|c|c|c|c|}
\hline DENTIFICATION & DELAY & trans. & ENERGY & $X-5 E C$ & $x=$ SEC & cou & & FRAC, & FAST & KGS & No, PIS \\
\hline 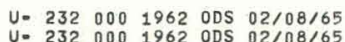 & 355.000 & 0.9471 & 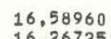 & 30.47 & 151 & $\begin{array}{l}\text { OPEN } \\
80259\end{array}$ & $\begin{array}{l}\text { SAPPLE } \\
72293\end{array}$ & $\begin{array}{l}\text { OF } \\
0,2946\end{array}$ & $\begin{array}{l}O P E N \\
969\end{array}$ & $\begin{array}{l}\text { MPLE } \\
959 \\
950\end{array}$ & \\
\hline a & 361.500 & $\begin{array}{l}0.9352 \\
0.9327\end{array}$ & $\begin{array}{l}26625 \\
99838\end{array}-100$ & $\begin{array}{l}37.53 \\
39.03\end{array}$ & $\begin{array}{l}151,38 \\
156,11\end{array}$ & $\begin{array}{l}55255 \\
59260\end{array}$ & $\begin{array}{l}52704 \\
52567\end{array}$ & $\begin{array}{l}0.2976 \\
0.3000\end{array}$ & $\begin{array}{l}746 \\
719\end{array}$ & $\begin{array}{l}739 \\
712\end{array}$ & 6 \\
\hline $\begin{array}{l}502 / 0 \\
502 / 0\end{array}$ & $\begin{array}{l}365,000 \\
368,500\end{array}$ & $\begin{array}{l}0.9171 \\
0.9204\end{array}$ & $\begin{array}{l}.69303 \\
.6934\end{array}$ & $\begin{array}{l}48.48 \\
46.50\end{array}-50$ & $\begin{array}{l}192,04 \\
192,46\end{array}$ & $\begin{array}{l}18585 \\
58573\end{array}$ & $\begin{array}{l}68544 \\
51268\end{array}$ & 0.3029 & 903 & 894 & \\
\hline $02 / 108 / 65$ & 2,000 & 0,9077 & 15,10799 & 54.25 & 210.86 & $\begin{array}{l}28575 \\
77500\end{array}$ & $\begin{array}{l}17268 \\
66902\end{array}$ & 0.3088 & 875 & 866 & \\
\hline 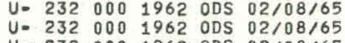 & $\begin{array}{l}376,000 \\
380,000\end{array}$ & $\begin{array}{l}0,9016 \\
0,8802\end{array}-2$ & $\begin{array}{l}14,78825 \\
14,47856\end{array}$ & $\begin{array}{l}58.03 \\
71.55\end{array}-5$ & $\begin{array}{l}2233.16 \\
272.26\end{array}-\mathrm{l}$ & $\begin{array}{l}75906 \\
75755\end{array}-100$ & $\begin{array}{l}55084 \\
63405\end{array}-100$ & $\begin{array}{l}0,3121 \\
0,3154\end{array}$ & $\begin{array}{l}874 \\
810\end{array}$ & $\begin{array}{l}865 \\
801\end{array}-1250$ & \\
\hline 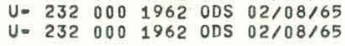 & $\begin{array}{l}384,000 \\
386,500\end{array}$ & $\begin{array}{l}0.8419 \\
0,8210\end{array}$ & $\begin{array}{l}14,17849 \\
13,9956\end{array}$ & $\begin{array}{r}96.44 \\
1110.54\end{array}$ & $\begin{array}{l}363.15 \\
43.53\end{array}-13$ & $\begin{array}{l}76210 \\
18443\end{array}$ & $\begin{array}{l}61014 \\
14399\end{array}$ & $\begin{array}{l}0,3187 \\
0,3208\end{array}-100$ & $\begin{array}{l}8344 \\
237\end{array}$ & $\begin{array}{l}826 \\
234\end{array}$ & \\
\hline S $02 / 08 / 65$ & 387,500 & 7980 & 352 & 126.48 & (5) & 18494 & 14033 & 0,3216 & 208 & 206 & \\
\hline 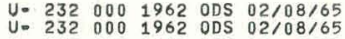 & $\begin{array}{l}388,500 \\
389,500\end{array}$ & $\begin{array}{l}0,7802 \\
0.7597\end{array}$ & $\begin{array}{l}13,85194 \\
13,78090\end{array}$ & $\begin{array}{l}139,09 \\
154: 05\end{array}$ & $\begin{array}{l}517: 66 \\
571 ; 86\end{array}-10$ & $\begin{array}{l}18476 \\
18048\end{array}$ & $\begin{array}{l}13708 \\
13038\end{array}$ & $\begin{array}{l}0.3225 \\
0: 3233\end{array}-3$ & $\begin{array}{l}183 \\
184\end{array}$ & 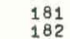 & \\
\hline 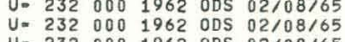 & $\begin{array}{l}390.500 \\
391.500\end{array}$ & $\begin{array}{l}0.7272 \\
0.7211\end{array}-\mathrm{l}-2$ & $\begin{array}{l}13 \\
13,7104046\end{array}$ & $\begin{array}{l}178.54 \\
183.51\end{array}$ & $\begin{array}{l}661.08 \\
677.00\end{array}$ & $\begin{array}{l}\begin{array}{l}18457 \\
18258\end{array} \\
-\end{array}$ & $\begin{array}{l}212763 \\
12518\end{array}-\mathrm{l}$ & $\begin{array}{l}0.3241 \\
0,3249\end{array}-32$ & $\begin{array}{l}{ }_{201}^{805} \\
201\end{array}$ & 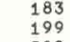 & \\
\hline 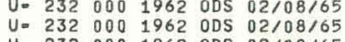 & $\begin{array}{l}392,500 \\
393,500 \\
500\end{array}$ & $\begin{array}{l}0.6810 \\
0.6581\end{array}-651$ & $\begin{array}{l}133,57104 \\
13,50215\end{array}$ & $\begin{array}{l}215,33 \\
234.53\end{array}$ & $\begin{array}{l}793.25 \\
861.77\end{array} \mathrm{~V}$ & 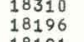 & $\begin{array}{l}11857 \\
11386 \\
1136\end{array}$ & $\begin{array}{l}0.3258 \\
0.3266\end{array}$ & $\begin{array}{l}212 \\
161 \\
205\end{array}$ & $\begin{array}{l}209 \\
159 \\
130\end{array}$ & \\
\hline 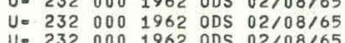 & $\begin{array}{l}394.500 \\
395 \\
305\end{array}$ & $\begin{array}{l}0.0020 \\
0.5452\end{array}$ & $\begin{array}{l}13.43599 \\
13.3659 \\
13\end{array}$ & $\begin{array}{l}284.44 \\
339.98 \\
3998\end{array}-9$ & $\begin{array}{l}1042.54 \\
1242.95\end{array}-92$ & $\begin{array}{l}18191 \\
17853\end{array}$ & $\begin{array}{c}10413 \\
9255 \\
925\end{array}$ & $\begin{array}{l}0,3274 \\
0,3283\end{array}-3284$ & $\begin{array}{l}185 \\
212 \\
212\end{array}$ & 210 & \\
\hline 0001962 ODS $02 / 08 / 65$ & 397.500 & 402 & 3,23178 & 509.6 & 3,80 & 18284 & 003 & & 1944 & & \\
\hline 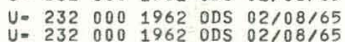 & $\begin{array}{l}398.500 \\
399.500\end{array}$ & $\begin{array}{l}0.2195 \\
0.2122\end{array}$ & $\begin{array}{l}13.16546 \\
13.09963\end{array}$ & $\begin{array}{l}639.61 \\
685.91\end{array}$ & $\begin{array}{l}2320,77 \\
31446\end{array}$ & $\begin{array}{l}18178 \\
18165\end{array}$ & $\begin{array}{l}5521 \\
3655\end{array}$ & $\begin{array}{l}0.3308 \\
0.3316\end{array}$ & 161 & 59 & \\
\hline & & & 13.03429 & 1124 & & 18307 & t & & & 193 & \\
\hline 21 & 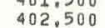 & & $\begin{array}{l}12,90508 \\
12,908\end{array}$ & 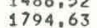 & 97 & $\begin{array}{lll}18032 \\
18032\end{array}$ & $\begin{array}{l}1204 \\
607\end{array}$ & 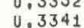 & 92 & 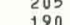 & \\
\hline 21 & 3. & & 12,84120 & 260 & & 7878 & 382 & 9 & & 94 & \\
\hline 196 & $\begin{array}{l}4.500 \\
4.500 \\
.500\end{array}-30$ & 11 & $\begin{array}{l}12,7178 \\
12,71484\end{array}$ & 28. & 8 & 88020 & $\begin{array}{l}244 \\
2.22\end{array}$ & 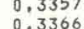 & & & \\
\hline $02 / 0$ & 406.500 & 153 & 12.65236 & 2342 . & & 17715 & 257 > 301 & 4 & & & \\
\hline 02108 & 407.50 & 0273 & 12,59034 & 2017.5 & 58,88 & 17635 & 458 & 0,3382 & 206 & 204 & \\
\hline 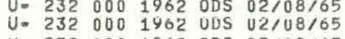 & $\begin{array}{l}408,500 \\
409,500\end{array}$ & $\begin{array}{l}0.0737 \\
0,1467\end{array}$ & $\begin{array}{l}12,52877 \\
12,46765\end{array}$ & $\begin{array}{l}1461,98 \\
1075,78\end{array}$ & $\begin{array}{l}1774.83 \\
798.54\end{array}-5$ & 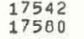 & $\begin{array}{l}1228 \\
2452\end{array}$ & $\begin{array}{l}0,3391 \\
0,3399\end{array}$ & $\begin{array}{l}188 \\
150\end{array}$ & $\begin{array}{l}186 \\
149\end{array}$ & \\
\hline $\begin{array}{l}232 \\
232 \\
2300\end{array}$ & $\begin{array}{l}410.500 \\
411.500\end{array}$ & $\begin{array}{l}0,2489 \\
0,3615\end{array}$ & $\begin{array}{l}12,40698 \\
12,34676\end{array}$ & $\begin{array}{l}779.43 \\
570.29\end{array}-1$ & $\begin{array}{l}\begin{array}{l}2745.42 \\
2003.89\end{array} \\
200\end{array}$ & $\begin{array}{l}17663 \\
17415\end{array}$ & $\begin{array}{l}4180 \\
5985\end{array}$ & $\begin{array}{l}0,3407 \\
0,3415\end{array}$ & $\begin{array}{l}167 \\
177\end{array}$ & $\begin{array}{l}166 \\
175\end{array}$ & \\
\hline 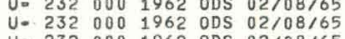 & $\begin{array}{l}412,500 \\
413,500 \\
4150\end{array}$ & $\begin{array}{l}0.4488 \\
0,5342 \\
045\end{array}$ & $\begin{array}{l}12,28697 \\
12,22761\end{array}$ & $\begin{array}{l}450.33 \\
351.41\end{array}$ & $\begin{array}{l}1578.55 \\
1228.82\end{array}$ & $\begin{array}{l}17563 \\
17331\end{array}$ & $\begin{array}{l}7477 \\
8802\end{array}$ & $\begin{array}{l}0,3424 \\
0,3432\end{array}$ & $\begin{array}{l}201 \\
164\end{array}$ & $\begin{array}{l}\frac{199}{162} \mathrm{~V} \mathrm{~V} \mathrm{~V} \\
162\end{array}$ & \\
\hline $232000196200502 / 08 / 65$ & 415.500 & 0.6622 & 12.11018 & 231.01 & $\begin{array}{l}10103.42 \\
803.91\end{array}$ & $\begin{array}{l}17454 \\
17393\end{array}$ & 10951 & 0 & $\begin{array}{l}168 \\
153\end{array}$ & & \\
\hline & & & & & & & & & & 194 & \\
\hline 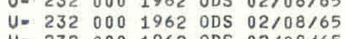 & $\begin{array}{l}417.500 \\
418,500\end{array}$ & 0.7496 & $\begin{array}{l}11.99443 \\
11.93718\end{array}$ & $\begin{array}{l}161,51 \\
148,46\end{array}$ & $\begin{array}{ll}512,94 & 5,35 \\
512 & \end{array}$ & $\begin{array}{l}17486 \\
17436\end{array}$ & $\begin{array}{l}12464 \\
12720\end{array}$ & $\begin{array}{l}0.3465 \\
0.3474\end{array}$ & $\begin{array}{l}165 \\
194\end{array}$ & ${ }_{103}^{163}$ & \\
\hline 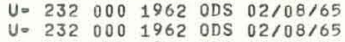 & $\begin{array}{l}419,500 \\
420,500\end{array}$ & $\begin{array}{l}0 \\
0\end{array}$ & $\begin{array}{l}11,88033 \\
11,82389\end{array}$ & $\begin{array}{l}128.71 \\
111633\end{array}-330$ & & $\begin{array}{l}17252 \\
17303\end{array}$ & $\begin{array}{l}13036 \\
13367\end{array}$ & ${ }^{2}$ & $\begin{array}{l}191 \\
213\end{array}$ & $\begin{array}{l}189 \\
211\end{array}$ & \\
\hline 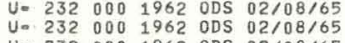 & $\begin{array}{l}421.500 \\
422.500\end{array}$ & $\begin{array}{l}0.8336 \\
0.8584\end{array}$ & $\begin{array}{l}11,76766 \\
11.71222\end{array}$ & $\begin{array}{l}102.00 \\
85.56\end{array}$ & $\begin{array}{l}349,90 \\
292.80\end{array}$ & $\begin{array}{l}17128 \\
16999\end{array}-V$ & $\begin{array}{l}13575 \\
13873\end{array}$ & 0 , & $\begin{array}{l}177 \\
170\end{array}$ & 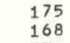 & \\
\hline ODS $02 / 08 / 65$ & $\begin{array}{l}425.000 \\
428.500\end{array}$ & 0. & $\begin{array}{l}11.57463 \\
11.38652\end{array}$ & $\begin{array}{c}63 \\
52\end{array}$ & $\begin{array}{l}21 \\
17\end{array}$ & $\begin{array}{l}67719 \\
50471\end{array}$ & $\begin{array}{l}57478 \\
43699\end{array}-109$ & 0. & 85 & $\begin{array}{l}730 \\
480\end{array}$ & \\
\hline 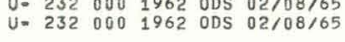 & $\begin{array}{l}432,000 \\
436,000\end{array}$ & $\begin{array}{l}0.9268 \\
0,9509\end{array}-3$ & $\begin{array}{l}11,20276 \\
10,99815\end{array}$ & $\begin{array}{l}42,58 \\
28,19\end{array}$ & $\begin{array}{l}12,52 \\
93,49\end{array}$ & $\begin{array}{l}67297 \\
65770\end{array}$ & $\begin{array}{l}59297 \\
59464\end{array}$ & $\begin{array}{l}0,3586 \\
0,3619\end{array}-2$ & $\begin{array}{l}7599 \\
682\end{array}$ & 年 615 & \\
\hline 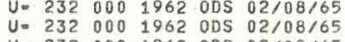 & $\begin{array}{l}440,000 \\
444,500\end{array}$ & & $\begin{array}{l}10,79909 \\
10,58154\end{array}$ & $\begin{array}{l}37 \\
29\end{array}$ & & $\begin{array}{l}\begin{array}{l}65843 \\
80306\end{array} \\
80\end{array}$ & & & $\begin{array}{l}6.6 \\
8\end{array}$ & $\begin{array}{l}632 \\
826\end{array}$ & ${ }_{0}^{8}$ \\
\hline 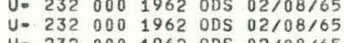 & $\begin{array}{l}\begin{array}{l}449,000 \\
453,000\end{array} \\
\end{array}$ & 0 : & $\begin{array}{l}10.37050 \\
10.18817\end{array}$ & $\begin{array}{l}24,06 \\
19,84\end{array}$ & $\begin{array}{l}77,49 \\
63 ; 34\end{array}-13$ & 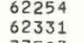 & 57190 & $\begin{array}{l}727 \\
760\end{array}$ & $\begin{array}{l}716 \\
701\end{array}$ & $\begin{array}{l}709 \\
694\end{array}$ & \\
\hline 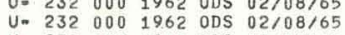 & $\begin{array}{l}457.500 \\
462.500\end{array}$ & $\begin{array}{l}0.9642 \\
0.9737\end{array}$ & $\begin{array}{l}9.98873 \\
9.77393\end{array}$ & 14: 20. & $\begin{array}{l}64,64 \\
46,79\end{array}$ & $\begin{array}{l}77503 \\
76481\end{array}-10$ & & & $\begin{array}{l}891 \\
890\end{array}$ & ${ }_{881}^{882}$ & \\
\hline 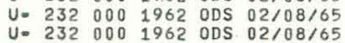 & $\begin{array}{l}467,000 \\
472,000\end{array}$ & $::-1-1$ & $\begin{array}{l}9.58647 \\
9.38444\end{array}$ & $\begin{array}{l}15 \\
17 \\
17\end{array}$ & 50 & $\begin{array}{l}60956 \\
89756\end{array}$ & $\begin{array}{l}56465 \\
82882\end{array}-125$ & & $\begin{array}{l}662 \\
935\end{array}$ & $\begin{array}{l}605 \\
655 \\
926\end{array}$ & \\
\hline 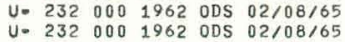 & $\begin{array}{l}477.500 \\
482.500\end{array}$ & 0.9652 & $\begin{array}{l}8.16950 \\
8.9045\end{array}$ & $\begin{array}{l}16.81 \\
19.88\end{array}$ & $\begin{array}{l}50.91 \\
54,58 \\
50\end{array}$ & $\begin{array}{l}73849 \\
73748\end{array}$ & $\begin{array}{c}00 \\
67\end{array}$ & $\begin{array}{l}0.3963 \\
0.4005\end{array}$ & 19 & $\begin{array}{l}811 \\
897\end{array}$ & \\
\hline 001962 ODS 021 & 488,000 & 0.9766 & 8.7 & 13 & 34 & 76 & 79 & 0. & 1027 & 1016 & \\
\hline 21 & 000 & & $\begin{array}{l}8,58 \\
8,39 \\
8,19\end{array}$ & & & & & & 1003 & 993 & \\
\hline 021 & & & 8,27981 & & & 13557 & & & 37 & 175 & \\
\hline 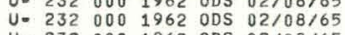 & $\begin{array}{l}504.500 \\
505\end{array}$ & 0 & $\begin{array}{l}8,21429 \\
8,21429\end{array}$ & & 10 & 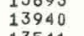 & $\begin{array}{lll}12878 \\
1283\end{array}$ & 0.8 & $1 / 1 / 2+3$ & $\begin{array}{l}109 \\
129\end{array}$ & \\
\hline $\begin{array}{lll}232 & 000 & 1962 \\
232 & 000 & 1962\end{array}$ & $\begin{array}{l}505.500 \\
506.500\end{array}$ & $0.97>-97$ & $\begin{array}{l}8.18183 \\
8.14955\end{array}$ & $\begin{array}{l}13 \\
12\end{array}$ & $\begin{array}{l}38,81 \\
35.08\end{array}$ & $\begin{array}{l}41 \\
56\end{array}$ & 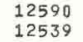 & $0:$ & $\begin{array}{l}170 \\
119\end{array}$ & $\begin{array}{l}168 \\
118\end{array}$ & \\
\hline 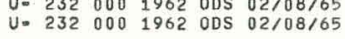 & $\begin{array}{l}507.500 \\
508.500\end{array}$ & $\begin{array}{l}0.9794 \\
0.9620\end{array}$ & $\begin{array}{l}8.17747 \\
8,08557\end{array}$ & $\begin{array}{l}\begin{array}{l}11.64 \\
21,73\end{array} \\
21\end{array}$ & $\begin{array}{l}33.16 \\
61.79\end{array}$ & 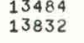 & $\begin{array}{l}125 \\
126\end{array}-\mathrm{l} \mathrm{V}$ & $\begin{array}{l}0,4212 \\
0,4221\end{array}-1$ & $\begin{array}{c}195 \\
143\end{array}$ & $\frac{3}{2}$ & \\
\hline 20001962 ODS $02 / 08 / 65$ & 500 & & 8.05386 & & & 13630 & & t & 183 & 181 & \\
\hline 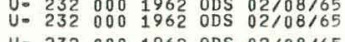 & $\begin{array}{l}510.500 \\
511.500\end{array}$ & $\begin{array}{l}0.0418 \\
0.9751\end{array}$ & $\begin{array}{l}8,0234 \\
7,99100 \\
79608\end{array}$ & 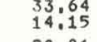 & $\left\{\begin{array}{l}27 \\
00\end{array}\right.$ & $\begin{array}{l}\frac{14116}{13208}-20 \\
1320\end{array}$ & 年12663 & $0:$ & 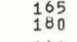 & $\begin{array}{l}163 \\
178\end{array}$ & \\
\hline 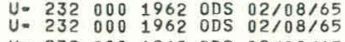 & $\begin{array}{l}512.500 \\
513.500\end{array}$ & $\begin{array}{l}0.9645 \\
0: 9387\end{array}$ & $\begin{array}{l}77,95985 \\
7: 92888 \\
9\end{array}$ & $\begin{array}{l}2000 \\
35 ; 4\end{array}$ & $\begin{array}{l}56: 45 \\
99: 82\end{array}-120$ & $\begin{array}{l}{ }_{13950}^{13930} \\
13958\end{array}$ & $\begin{array}{l}12803 \\
12480\end{array}$ & $\begin{array}{l}0,4254 \\
0,4202\end{array}$ & $\begin{array}{l}161 \\
184\end{array}$ & $\begin{array}{l}160 \\
183\end{array}$ & \\
\hline 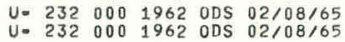 & $\begin{array}{l}514,500 \\
515,500\end{array}$ & $\begin{array}{l}0,9511 \\
0,9658\end{array}$ & $\begin{array}{l}7: 8908 \\
7: 6747 \\
7: 6747\end{array}$ & $\begin{array}{l}28.0 \\
19.5\end{array}$ & $\begin{array}{l}78.92 \\
54.97\end{array}$ & $\begin{array}{l}13556 \\
13422\end{array}$ & 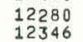 & $0:$ & $\begin{array}{l}172 \\
124\end{array}$ & 1 & \\
\hline $\begin{array}{l}02 / 88 / 65 \\
02 / 08 / 65\end{array}$ & $\begin{array}{l}516,500 \\
517500\end{array}$ & 98. & $\begin{array}{l}7,8304 \\
7: 0678 \\
7: 0678\end{array}$ & 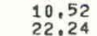 & $: 44$ & $\begin{array}{l}12990 \\
12786\end{array}$ & $\begin{array}{l}12142 \\
11704\end{array}$ & $\begin{array}{l}0: 4287 \\
0 ; 4295\end{array}$ & & $\begin{array}{l}128 \\
180\end{array}$ & \\
\hline 20001962 ODS 021 & 5. & & 7.77669 & & & 13038 & & 0. & & & \\
\hline $\begin{array}{l}2320001962 \text { ODS } 02 / 08 / 165 \\
232000196200 S 02 / 08 / 65\end{array}$ & $\begin{array}{l}519.500 \\
520.500\end{array}$ & 9675 & 7,746 & & 1 & $\begin{array}{l}12885 \\
13273\end{array}$ & 11873 & 0. & $\begin{array}{l}159 \\
130\end{array}$ & 157 & \\
\hline & & & & & & & & & & $\begin{array}{l}129 \\
167\end{array}-\mathrm{J}$ & \\
\hline & & & & & & & & & & 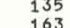 & \\
\hline & & & & & & & & & & & \\
\hline $\begin{array}{l}19 \\
19\end{array}$ & $\begin{array}{l}525,500 \\
526.500\end{array}$ & 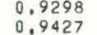 & $\begin{array}{l}7,57 \\
7,54\end{array}$ & & 112 & & & & & 3 & \\
\hline $\begin{array}{llll}232 & 000 & 1962 & 0 \\
232 & 000 & 1962 & 01\end{array}$ & 500 & $\begin{array}{l}9297 \\
9445\end{array}$ & 48519 & & & & & & & & \\
\hline 20001962 oDS $02 / 08 / 6$ & .500 & & 7.456 & & & 12665 & 119 & & 146 & & \\
\hline & & & & & & & & & & & \\
\hline 021 & $\begin{array}{r}532.5 \\
533.5\end{array}$ & & & & & & & & & 1 & \\
\hline 0210 & & & & & & & & & & & \\
\hline 1962 oD 1900 & .500 & 97 & 7.26 & 13,0 & & & $\begin{array}{l}113 \\
114\end{array}$ & $\begin{array}{l}0.4445 \\
0.4453\end{array}$ & & 10 & \\
\hline 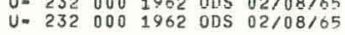 & 500 & 1513 & & & & & & $\begin{array}{l}4461 \\
4470\end{array}$ & & & \\
\hline
\end{tabular}




\begin{tabular}{|c|c|c|c|c|c|c|c|c|c|c|c|c|c|c|c|}
\hline \multicolumn{4}{|c|}{ IDENTIFICATION } & \multirow[t]{2}{*}{ DATE } & \multirow[t]{2}{*}{ DELAY } & \multirow{2}{*}{ TRANS, } & \multirow{2}{*}{ ENERGY } & \multirow{2}{*}{$x=S E C$} & \multirow{2}{*}{$\begin{array}{l}x=S E C \\
\text { ROOT E }\end{array}$} & \multicolumn{2}{|c|}{ COUNTS } & & AST & BKGS & NO, PTS \\
\hline U. 232 & 000 & 1962 & ODS & & & & & & & OPEN & SAMPLE & OF OCT & OPEN & SAMPLE & AVE, \\
\hline 232 & 000 & 1962 & ODS & $02 / 08 / 65$ & 540.500 & 0.9782 & 7.15651 & $\begin{array}{l}24,26 \\
12,35\end{array}$ & 65.01 & 12325 & 11232 & 0,4478 & 149 & 148 & \\
\hline U. 232 & 000 & 1962 & ODS & $02 / 08 / 65$ & 541.500 & 0.9413 & 7.13010 & 33,90 & $\begin{array}{l}33,05 \\
90,52\end{array}$ & 12422 & 11560 & 0,4486 & 160 & 159 & 2 \\
\hline U. 232 & 000 & 1962 & ODS & $02 / 08 / 65$ & 542.500 & 0.9480 & 7.10384 & 29.94 & 79,80 & 12340 & $\begin{array}{l}10949 \\
11123\end{array}$ & $\begin{array}{l}0,4494 \\
0,4503\end{array}$ & $\begin{array}{l}158 \\
195\end{array}$ & 156 & 2 \\
\hline U. 232 & 000 & 1962 & ODS & $02 / 08 / 65$ & 545.000 & 0.9513 & 7.03881 & 27,98 & 74.23 & 49210 & 44474 & 0,4523 & $\begin{array}{l}195 \\
660\end{array}$ & $\begin{array}{l}193 \\
653\end{array}$ & $\begin{array}{l}2 \\
8\end{array}$ \\
\hline$u=232$ & 000 & 1962 & ODS & $02 / 08 / 65$ & 549.000 & 0.9469 & 6,93662 & 30,58 & 80.54 & 48028 & 43112 & 0,4557 & 588 & 583 & 8 \\
\hline$U=232$ & 000 & 1962 & ODS & $02 / 08 / 65$ & 553,000 & 0,9422 & 6.83663 & 33.40 & 87,33 & 47409 & 42193 & 0,4590 & 643 & 636 & 8 \\
\hline U. 232 & 000 & 1962 & ODS & $02 / 08 / 65$ & 557,000 & 0,9369 & 6.73879 & 36.52 & 94,80 & 46655 & 41293 & 0,4623 & 616 & 610 & 8 \\
\hline 232 & 000 & 1962 & ODS & $02 / 08 / 65$ & 561.000 & 0,9168 & .64304 & 48,67 & 125,44 & 46486 & 40352 & 0,4656 & 639 & 633 & 8 \\
\hline$v=232$ & 000 & 1962 & ODS & $02 / 08 / 65$ & 565.500 & 0,9204 & 6.53774 & 46.52 & 118,94 & 56688 & 49489 & 0,4694 & 791 & 783 & 10 \\
\hline$U=232$ & 000 & 1962 & ODS & $02 / 08 / 65$ & 570.000 & 0.8887 & 6.43492 & 66.16 & 167,82 & 44563 & 37614 & 0.4731 & 656 & 649 & 8 \\
\hline $\begin{array}{l}U: \\
U=232 \\
U 232\end{array}$ & $\begin{array}{l}000 \\
000\end{array}$ & $\begin{array}{l}1962 \\
1962\end{array}$ & $\begin{array}{l}\text { ODS } \\
\text { ODS }\end{array}$ & $\begin{array}{l}02 / 08 / 65 \\
02 / 08 / 65\end{array}$ & $\begin{array}{l}572.500 \\
573.500\end{array}$ & $\begin{array}{l}0,8450 \\
0,8314\end{array}$ & $\begin{array}{l}6,37884 \\
6,35661\end{array}$ & $\begin{array}{r}94.42 \\
103.51\end{array}$ & $\begin{array}{l}238,47 \\
260: 98\end{array}$ & $\begin{array}{l}11074 \\
11151\end{array}$ & $\begin{array}{l}8893 \\
8812\end{array}$ & $\begin{array}{l}0,4752 \\
0,4760\end{array}$ & $\begin{array}{l}141 \\
152\end{array}$ & $\begin{array}{l}140 \\
150\end{array}$ & $\begin{array}{l}2 \\
2\end{array}$ \\
\hline$v=232$ & 000 & 1962 & ODS & $02 / 08 / 65$ & 574,500 & 0.8146 & 6,33450 & 114.93 & 289,25 & 11076 & 8578 & 0,4768 & 174 & 172 & 2 \\
\hline U. 232 & 000 & 1962 & ODS & $02 / 08 / 65$ & 575.500 & 0,8103 & 6.31251 & 117.90 & 296.23 & 11029 & 8497 & 0.4777 & 161 & 160 & 2 \\
\hline$U=232$ & 000 & 1962 & ODS & $02 / 08 / 65$ & 576.500 & 0.7826 & 6,29063 & 137.37 & 344.54 & 11265 & 8385 & 0,4785 & & 143 & 2 \\
\hline$U=232$ & 000 & 1962 & ODS & $02 / 08 / 65$ & 577.500 & 0,7348 & 6,26886 & 172.70 & 432,39 & 11265 & 7874 & 0,4793 & 174 & 172 & 2 \\
\hline U. 232 & 000 & 1962 & ODS & $02 / 08 / 65$ & 578,500 & 0,7043 & 6,24721 & 196,48 & 491,08 & 11079 & 7423 & 0.4802 & 146 & 144 & 2 \\
\hline$U=232$ & 000 & 1952 & ODS & $02 / 08 / 65$ & 79.500 & 0,6660 & 6.22566 & 227.83 & 568.48 & 10900 & 6907 & 0.4810 & 160 & 159 & 2 \\
\hline$U=232$ & & 1962 & ODS & $02 / 08 / 65$ & 580,500 & 0,6318 & 6.20423 & 257.35 & 641.02 & 10722 & 6446 & 0.4818 & 174 & 172 & 2 \\
\hline$v=232$ & 000 & 1962 & ODS & $02 / 08 / 65$ & 581.500 & 0.5271 & 6.18291 & 358,92 & 892.47 & 10683 & 5358 & 0.4826 & 159 & 157 & 2 \\
\hline $\begin{array}{l}y=232 \\
y=232\end{array}$ & $\begin{array}{l}000 \\
000\end{array}$ & $\begin{array}{l}1962 \\
1962\end{array}$ & $\begin{array}{l}\text { ODS } \\
\text { ODS }\end{array}$ & $\begin{array}{l}02 / 08 / 65 \\
02 / 08 / 65\end{array}$ & $\begin{array}{l}582,500 \\
583,500\end{array}$ & $\begin{array}{l}0,4538 \\
0.3345\end{array}$ & 6.16170 & 442,83 & 1099,22 & 10687 & 4615 & 0.4835 & 108 & 107 & 2 \\
\hline $\begin{array}{l}=232 \\
u=232\end{array}$ & 000 & 1962 & ODS & $02 / 08 / 65$ & 584,500 & $\begin{array}{l}0.3345 \\
0.2388\end{array}$ & $\begin{array}{l}6.14060 \\
6.11961\end{array}$ & 613.84 & 1521.11 & 10810 & 3440 & 0,4843 & $\begin{array}{l}165 \\
140\end{array}$ & $\begin{array}{l}163 \\
138\end{array}$ & $\begin{array}{l}2 \\
2\end{array}$ \\
\hline$u=232$ & 000 & 1962 & ODS & $02 / 08 / 65$ & 585.500 & 0.1315 & $\begin{array}{l}0.11961 \\
6.09872\end{array}$ & $\begin{array}{r}802,75 \\
1137,31\end{array}$ & $\begin{array}{l}1985,82 \\
2808,64\end{array}$ & $\begin{array}{l}10733 \\
10776\end{array}$ & $\begin{array}{l}2438 \\
1347\end{array}$ & $\begin{array}{l}0,4851 \\
0.4860\end{array}$ & $\begin{array}{l}140 \\
159\end{array}$ & $\begin{array}{l}138 \\
157\end{array}$ & $\begin{array}{l}2 \\
2\end{array}$ \\
\hline U. 232 & 000 & 1962 & ODS & $02 / 08 / 65$ & 586,500 & 0.0711 & 6,07794 & 1482.14 & 3653.98 & 10715 & 724 & 0,4868 & 154 & 153 & 2 \\
\hline 0.232 & 000 & 1962 & UDS & $02 / 08 / 65$ & 587,500 & 0,0435 & 6,05727 & 1757.55 & 4325,60 & 10457 & 432 & 0. & 153 & 152 & 2 \\
\hline U. 232 & 000 & 1962 & ODS & $02 / 08 / 65$ & 588,500 & 0,0248 & 6.03670 & 2073.11 & 5093.58 & 10614 & 249 & 0.4 & 175 & 173 & 2 \\
\hline$y=232$ & 000 & & ODS & $02 / 08 / 65$ & 589.500 & 0,0288 & 6.01624 & 1989.13 & 4878.94 & 10504 & 287 & 0.4893 & 141 & 140 & 2 \\
\hline$U=232$ & 000 & 1962 & ODS & $02 / 08 / 65$ & 590,500 & 0,0197 & 5,99588 & 2200,18 & 5387.47 & 10656 & 200 & 0,4901 & 155 & 154 & 2 \\
\hline$U=232$ & 000 & 1962 & ODS & $02 / 08 / 65$ & 591.500 & 0,0099 & 5.97562 & 2585,96 & 6321.41 & 10366 & 97 & 0,4 & 176 & 174 & 2 \\
\hline & 000 & 1962 & ODS & $02 / 08 / 65$ & 592,500 & 0.0136 & 95547 & 240 & 5875,61 & 10551 & 136 & 0,4 & 145 & 143 & 2 \\
\hline$v=232$ & 000 & 1962 & ODS & $02 / 08 / 65$ & 593,500 & 0.0127 & 542 & 2445,23 & 5957.25 & 10658 & 129 & 0.4926 & 148 & 147 & 2 \\
\hline$u=232$ & 000 & 1962 & ODS & $02 / 08 / 65$ & 594.500 & 0.0191 & 5.91546 & 2219,74 & 5398.80 & 10391 & 188 & 0,4934 & 172 & 171 & 2 \\
\hline $\begin{array}{l}U=232 \\
032\end{array}$ & 000 & 1962 & ODS & $02 / 08 / 65$ & 595,500 & 0.0495 & 5.89561 & 1684.85 & 4090.96 & 10160 & 478 & 0.4 & 154 & 153 & 2 \\
\hline U. 232 & 000 & 1962 & ODS & $02 / 08 / 65$ & 596.500 & 0.1279 & 5.87586 & 1152.65 & 2794.03 & 10290 & 1252 & 0 & 139 & 137 & 2 \\
\hline U. 232 & 000 & 1962 & ODS & $02 / 08 / 65$ & 597,500 & 0.2157 & 85621 & 859.66 & 2080,34 & 10265 & 2106 & 0,4959 & 163 & 161 & 2 \\
\hline$v=232$ & 000 & 1962 & ODS & $02 / 08 / 65$ & 598,5 & 0,3356 & 5,83666 & 611.98 & 1478,49 & 10164 & 3245 & 0,4 & 146 & 144 & 2 \\
\hline$v=232$ & 000 & 1962 & ODS & $02 / 08 / 65$ & 599,500 & 0.4324 & 5,81720 & 469.89 & 1133.32 & 10141 & 4172 & 0.4976 & 152 & 150 & 2 \\
\hline u. 232 & UOU & 1962 & ODS & $02 / 08 / 65$ & 600,500 & 0.5067 & 5.79784 & 381,10 & 917.63 & 10297 & 4964 & 0,4984 & 175 & 173 & 2 \\
\hline$u=232$ & 000 & 1962 & ODS & $02 / 08 / 65$ & 01,500 & 0.5793 & 5.77858 & 305.95 & 735.47 & 10175 & 5609 & 92 & 163 & 161 & 2 \\
\hline & 000 & 1962 & ODS & $02 / 08 / 65$ & 02.500 & 0.6323 & 5.75942 & 256.88 & 616.49 & 10168 & 6119 & 0.5 & 164 & 162 & 2 \\
\hline$u=232$ & 000 & 1962 & ODS & & 10 & 0.66 & 5,74035 & 226.57 & 542 & 10112 & 64 & & 157 & 155 & 2 \\
\hline $\begin{array}{r}U=232 \\
y=233\end{array}$ & 000 & 1962 & ODS & $02 / 08 / 65$ & 4.500 & 0.7226 & 5.72137 & 182.13 & 435.65 & 10116 & 6956 & 0.5 & 164 & 162 & 2 \\
\hline$U=232$ & 000 & 1962 & ODS & $02 / 08 / 65$ & 5,500 & .7465 & 5,70249 & 163.89 & 391.37 & 9992 & 7099 & 0.5 & 11 & & 2 \\
\hline$U=232$ & 000 & 1962 & ODS & $02 / 08 / 65$ & 36,500 & 0.7755 & 5,68370 & 142.52 & 339.77 & 10051 & 7419 & 0,5 & 14 & 148 & 2 \\
\hline U. 232 & 000 & 1962 & ODS & $02 / 08 / 65$ & 607,500 & 0,7911 & 5,66500 & 131.3 & 312.54 & 10022 & 7547 & 0.5 & 152 & 150 & 2 \\
\hline$u=232$ & 000 & 1962 & ODS & $02 / 08 / 65$ & 608,500 & 0,8026 & 5,64640 & 123,26 & 292,8 & 10226 & 7812 & 0,5 & 199 & 197 & 2 \\
\hline U. 232 & 000 & 1962 & $O D S$ & $02 / 08 / 65$ & 609.500 & 0.8359 & 5.62788 & 100,45 & 238,3 & 10034 & 798 & 0.5 & 180 & 178 & 2 \\
\hline u. 232 & 000 & 1962 & ODS & $02 / 08 / 65$ & 610.500 & 0.8439 & 5.60946 & 95,14 & 225,33 & 10057 & 8079 & 0.5067 & 146 & 144 & 2 \\
\hline$u=232$ & 000 & 1962 & ODS & $02 / 08 / 65$ & 13.500 & 0,8688 & 5,55474 & & & & & & & & 10 \\
\hline$v=232$ & 000 & & & & & & & & 112 & 4 & 50 & & 1008 & 998 & $?$ \\
\hline$v=232$ & 000 & 1962 & ODS & $02 / 08 /$ & 100 & 0.9 & 5220 & 3 & 88.65 & 56506 & 502 & & 1055 & 1044 & 12 \\
\hline$y=232$ & 000 & 1962 & ODS & $02 / 08$ & 10 & 9 & & & 70. & 54741 & 493 & & 909 & 900 & 12 \\
\hline$U=232$ & 000 & 1962 & ODS & $02 / 0$ & & & & & 57. & 53776 & 48925 & 0.5 & 973 & & 12 \\
\hline$U=232$ & 000 & 1962 & ODS & $02 / 081$ & 6,5 & 0.96 & 5,00214 & 21.41 & 47,89 & 113169 & 103661 & 0.53 & 2049 & 2028 & 26 \\
\hline$U=232$ & 000 & 1962 & ODS & $02 / 08 / 65$ & 660,000 & 0.9650 & & 20. & 43,81 & 116406 & 106877 & 0.5 & 2236 & 2214 & 28 \\
\hline$U=232$ & 000 & 1962 & ODS & $02 / 08 / 65$ & 674,000 & 9691 & 4.60228 & 17 & 37.68 & 110860 & 102 & 0.5 & 2478 & 2453 & 28 \\
\hline$u=232$ & 000 & 1962 & ODS & $02 / 0$ & 689,000 & 0.9629 & 4,40407 & 21 & 44,52 & 115266 & 10563 & 0.5 & 2794 & 2766 & 32 \\
\hline 32 & 000 & 1962 & ODS & $02 / 08 / 65$ & 705,500 & 0,9649 & 4,20048 & 20.04 & 41.07 & 111627 & 102542 & 0,5856 & 3082 & 3052 & 34 \\
\hline U. 232 & 000 & 1962 & ODS & 0 & 0 & 0,9681 & 9 & 18 & & 117998 & 108766 & 0.6001 & 3354 & 3320 & 36 \\
\hline$U=232$ & 000 & $196 ?$ & ODS & ก2/08/65 & 741.500 & 0,9688 & & 17 & & 35 & & & 3635 & & 38 \\
\hline 32 & 000 & 1962 & ODS & $02 / 0$ & & & & 18 & & 118 & & & & 4140 & 42 \\
\hline$U=232$ & 000 & 1962 & ODS & $02 / 081$ & 3.500 & 0,96 & 3.4057 & 17 & 31. & 116688 & 107602 & 0,65 & 4569 & 4523 & 46 \\
\hline$U=232$ & 000 & 1962 & ODS & $02 / 0$ & & & & 15 & 27 & 113107 & 104789 & 0.67 & 5317 & 5264 & 52 \\
\hline$U=232$ & 000 & 1962 & ODS & & 0 & 0.96 & 3. & 18. & 31.93 & 95131 & 87644 & 0.6 & 5931 & 587 & 54 \\
\hline$U=232$ & 000 & 1962 & ODS & $02 / 08 / 65$ & 863.500 & 0.9692 & 2.80393 & & 29,37 & 81929 & 7560 & & 8226 & 8144 & 62 \\
\hline U. 232 & 000 & 1962 & ODS & $02 / 08 / 65$ & 896.500 & 0,9687 & .60131 & 17.85 & 28,79 & 61710 & 56909 & 0,7441 & 12713 & 12586 & 70 \\
\hline
\end{tabular}


RUN 1963

\begin{tabular}{|c|c|c|c|c|c|c|c|c|c|c|c|}
\hline IDENTIFICATION & DELAY & trans. & ENERGY & $x-5 E C$ & $\begin{array}{l}\text { X.SEC } \\
\text { ROOT E }\end{array}$ & OPEN & $\begin{array}{l}\text { ITS } \\
\text { SAMPLE }\end{array}$ & $\begin{array}{l}\text { FRAC, } \\
\text { OF OCT }\end{array}$ & $\underset{\substack{\text { FAST } \\
\text { OPEN }}}{2}$ & $\begin{array}{l}\text { KGS } \\
\text { SAMPLE }\end{array}$ & NO $_{\text {AVE, }}^{\text {PTS }}$ \\
\hline 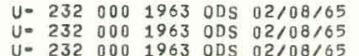 & $\begin{array}{l}15.050 \\
16.002 \\
11.005\end{array}$ & $\begin{array}{l}0,9868 \\
1: 0085 \\
1.0051\end{array}$ & $\begin{array}{l}9202,70911 \\
8091.67847 \\
7370296\end{array}$ & $\begin{array}{r}29.47 \\
-18.79 \\
-11.32\end{array}$ & $\begin{array}{r}2826,80 \\
-1960.16\end{array}$ & $\begin{array}{l}21692 \\
1877 \\
190738\end{array}$ & $\begin{array}{l}20918 \\
18205\end{array}$ & $\begin{array}{l}0,0122 \\
0.0130 \\
0.138\end{array}$ & $\begin{array}{l}7776 \\
8721 \\
6070\end{array}$ & $\begin{array}{l}7776 \\
8421\end{array}$ & 2 \\
\hline 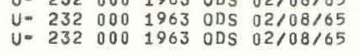 & $\begin{array}{l}11.000 \\
19: 050 \\
19.050\end{array}$ & $\begin{array}{l}1,0051 \\
1,012 \\
0.9912\end{array}$ & 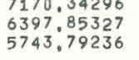 & $\begin{array}{l}-11,32 \\
-37.87 \\
19.50\end{array}$ & $\begin{array}{l}-3958.46 \\
-302 \% 33 \\
1477.94\end{array}$ & $\begin{array}{l}19308 \\
16458 \\
18353\end{array}$ & $\begin{array}{l}18964 \\
16595 \\
17776\end{array}$ & $\begin{array}{l}0.0138 \\
0.0146 \\
0.0154\end{array}$ & $\begin{array}{l}6079 \\
653 \\
5016\end{array}$ & $\begin{array}{l}6079 \\
6553 \\
5016\end{array}$ & \\
\hline $\begin{array}{l}0001963 \text { oDs } 02 / 08 / 65 \\
0001963 \text { ODS } 02 / 08 / 65\end{array}$ & $\begin{array}{l}20.050 \\
21.050\end{array}$ & $\begin{array}{l}0.9982 \\
0.9975\end{array}-\mathrm{l}$ & $\begin{array}{l}5185.13342 \\
4704.18604\end{array}$ & $\begin{array}{l}3.97 \\
5.49\end{array}$ & $\begin{array}{l}285.85 \\
376.58\end{array}$ & 18730 & $1826 \varepsilon$ & 0,0162 & 4583 & 583 & \\
\hline 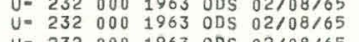 & $\begin{array}{l}21,0.050 \\
22,030\end{array}$ & $\begin{array}{l}0.9955 \\
0.9967\end{array}$ & $\begin{array}{l}\begin{array}{l}4704,18604 \\
4287,17786\end{array} \\
20\end{array}$ & $\begin{array}{l}5.49 \\
7.27\end{array}$ & $\begin{array}{l}377.58 \\
475.82\end{array}$ & $\begin{array}{l}20357 \\
19417\end{array}$ & 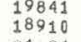 & $\begin{array}{l}0.0171 \\
0.0179\end{array}$ & $\begin{array}{l}2441 \\
2659 \\
269\end{array} \mathrm{~V}$ & 559 & \\
\hline 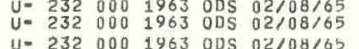 & $\begin{array}{l}233: 300 \\
24: 550 \\
23,550\end{array}$ & $\begin{array}{l}0: 9950 \\
1: 0076 \\
4.9810\end{array}$ & $\begin{array}{l}3839.51932 \\
3458: 48343\end{array}$ & $\begin{array}{l}11.17 \\
-16.75 \\
4140\end{array}$ & $\begin{array}{l}641.96 \\
-985: 27\end{array}$ & $\begin{array}{l}27498 \\
19968 \\
21971\end{array}$ & $\begin{array}{l}26084 \\
19658 \\
2069\end{array}$ & $\begin{array}{l}0.0189 \\
0.0199 \\
0.01997\end{array}$ & $\begin{array}{l}4335 \\
3196\end{array}$ & $\begin{array}{l}4335 \\
3196 \\
1964\end{array}$ & \\
\hline 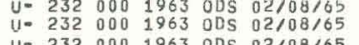 & $\begin{array}{l}26.550 \\
26.550\end{array}$ & $\begin{array}{l}0.489 \\
0.9730 \\
0.9730\end{array}$ & $\begin{array}{l}3193.05856 \\
2957.05601\end{array}$ & $\begin{array}{l}40,49 \\
60,78\end{array}$ & $\begin{array}{l}2288,23 \\
3305,26\end{array}$ & $\begin{array}{l}21571 \\
20865\end{array}$ & $\begin{array}{l}20694 \\
19834\end{array}$ & $\begin{array}{l}0.027 \\
0.0215 \\
0.0215\end{array}$ & $\begin{array}{l}1984 \\
1945\end{array}$ & 1844 1845 & \\
\hline 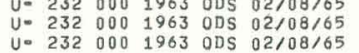 & $\begin{array}{l}27.500 \\
28: 550 \\
29.550\end{array}$ & $\begin{array}{l}0.9643 \\
1,0139 \\
0.967\end{array}$ & $\begin{array}{l}2746.28424 \\
2557.26929 \\
2387\end{array}$ & $\begin{aligned} 800.57 \\
-30.59\end{aligned}$ & $\begin{aligned} 4222.499 \\
-1546,92\end{aligned}$ & $\begin{array}{l}11066 \\
16569\end{array}$ & $\begin{array}{l}17964 \\
16216\end{array}-160$ & $\begin{array}{l}0.023 \\
0.0231 \\
0.0230\end{array}$ & $\begin{array}{l}2115 \\
2511\end{array}$ & $\begin{array}{l}2115 \\
2511\end{array}$ & \\
\hline & & 0.9941 & 2233,39871 & 13.10 & & 179 & & & & & \\
\hline $\begin{array}{l}02 / 18 / 15 \\
02 / 08 / 65\end{array}$ & 550 & $\begin{array}{l}0,9987 \\
0,9838\end{array}$ & $\begin{array}{l}2094,06406 \\
196737303\end{array}$ & $\begin{array}{r}22.87 \\
36,30\end{array}$ & $\begin{array}{l}131,36 \\
1610,10\end{array}$ & $\begin{array}{l}19959 \\
2144\end{array}$ & $\begin{array}{l}19476 \\
2059\end{array}$ & & $\begin{array}{l}1381 \\
1259\end{array}$ & $59^{\circ}-2>$ & \\
\hline 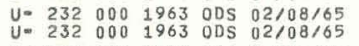 & $\begin{array}{l}33.550 \\
34: 550\end{array}$ & $\begin{array}{l}0,9901 \\
0,9667\end{array}$ & $\begin{array}{l}1851,84079 \\
1146,1941\end{array}$ & $\begin{array}{l}22,16 \\
25,12\end{array}$ & $\begin{aligned} 953,68 \\
3139.03\end{aligned}$ & $\begin{array}{l}21626 \\
22557\end{array}$ & $\begin{array}{l}20916 \\
21116 \\
2116\end{array}$ & 0.0272 & $\begin{array}{l}1332 \\
1068\end{array}$ & $\begin{array}{l}1332 \\
1308\end{array}$ & \\
\hline 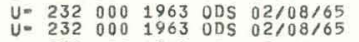 & $\begin{array}{l}35.550 \\
36,550\end{array}$ & $\begin{array}{l}0,9863 \\
0,9760\end{array}$ & $\begin{array}{l}1649.33731 \\
1560 ; 32094\end{array}$ & $\begin{array}{l}30,68 \\
53 ; 82\end{array}$ & $\begin{array}{l}1245: 97 \\
2125 ; 80\end{array}$ & $\begin{array}{l}22384 \\
22297\end{array}$ & $\begin{array}{l}21565 \\
21268\end{array}$ & $\begin{array}{l}0.0288 \\
0.0096\end{array}$ & 1122 & $\begin{array}{r}762 \\
1122 \\
1122\end{array}$ & \\
\hline 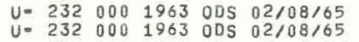 & $\begin{array}{l}37,550 \\
38: 550\end{array}$ & $\begin{array}{l}0,9921 \\
0,9945\end{array}$ & $\begin{array}{l}1478,32123 \\
1402,61969\end{array}$ & $\begin{array}{l}17,56 \\
12,23\end{array}$ & $\begin{array}{l}675.31 \\
488.09\end{array}$ & $\begin{array}{l}21686 \\
21725\end{array}$ & $\begin{array}{l}21021 \\
21109\end{array}$ & $\begin{array}{l}0.0304 \\
0.0312\end{array}$ & $\begin{array}{l}1157 \\
1301\end{array}$ & $\begin{array}{l}157 \\
151 \\
01\end{array}$ & \\
\hline 320001963 ODS $02 / 08 / 65$ & $39,5 ? 0$ & 0.9891 & 1332,58745 & 24.33 & 888.03 & 21874 & 21139 & 0.0320 & 1518 & 1518 & \\
\hline $\begin{array}{l}000 \quad 1963 \text { ODS } 02 / 08 / 65 \\
000 \\
00063\end{array}$ & $\begin{array}{l}40.550 \\
41.550\end{array}$ & $\begin{array}{l}0,9823 \\
0,9931\end{array}$ & $\begin{array}{l}1267.67223 \\
1207.38739\end{array}$ & $\begin{array}{l}39.66 \\
15,25\end{array}$ & & $\begin{array}{l}21722 \\
20413\end{array}$ & & & 32 & & \\
\hline 1963 & $\begin{array}{l}42.550 \\
43.550\end{array}$ & $\begin{array}{ll}0.9782 \\
0,9967\end{array}$ & $\begin{array}{l}1151 ; 30281 \\
11599.03716\end{array}$ & $\begin{array}{r}48,84 \\
7.40\end{array}$ & $\begin{array}{l}1657.31 \\
245.16\end{array}$ & $\begin{array}{l}18351 \\
1843\end{array}$ & $\begin{array}{l}17535 \\
17959\end{array}$ & $\begin{array}{l}0.0345 \\
0.0053\end{array}$ & 2376 & 2376 & \\
\hline 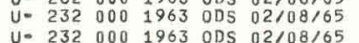 & $\begin{array}{l}44.550 \\
45.550\end{array}$ & $\begin{array}{l}0.9796 \\
0.9944\end{array}$ & $\begin{array}{l}1050.25142 \\
100464339\end{array}$ & $\begin{array}{r}45.67 \\
13.67\end{array}$ & $\begin{array}{l}1479: 18 \\
1493\end{array}$ & $\begin{array}{l}1049 \\
19757 \\
2063\end{array}$ & $\begin{array}{l}18959 \\
180037\end{array}$ & $\begin{array}{l}0,0361 \\
0.0369\end{array}$ & $\begin{array}{l}2206 \\
2467 \\
2356\end{array}$ & $\begin{array}{lll}22407 \\
2467 \\
2536\end{array}$ & \\
\hline 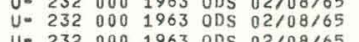 & $\begin{array}{l}46.550 \\
46.550\end{array}$ & $\begin{array}{l}0,944 \\
0,9967\end{array}$ & $\begin{aligned} 1004.64339 \\
961.94296\end{aligned}$ & $\begin{array}{l}12.40 \\
7.35\end{array}$ & 227.81 & 20197 & 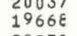 & $\begin{array}{l}0,0337 \\
0,0377\end{array}$ & $\begin{array}{l}2536 \\
3029\end{array}$ & $\begin{array}{l}2536 \\
3029\end{array}$ & \\
\hline $\begin{array}{l}02108 / 155 \\
02 / 08 / 65\end{array}$ & $\begin{array}{l}47,550 \\
48 ; 550\end{array}$ & $\begin{array}{l}0,9778 \\
0 ; 9792\end{array}$ & $\begin{array}{l}921,90814 \\
884,32159\end{array}$ & $\begin{array}{l}49,85 \\
46,71\end{array}$ & $\begin{array}{l}15133,70 \\
1389 ; 03\end{array}$ & $\begin{array}{l}21211 \\
20582\end{array}$ & $\begin{array}{l}20272 \\
19690\end{array}$ & $\begin{array}{l}0.0355 \\
0,0393\end{array}$ & $\begin{array}{l}2534 \\
2868\end{array}$ & 534 & \\
\hline $2 / 1$ & & 1,0028 & 848,987 & -6.10 & & 20535 & & & & & \\
\hline 年 & 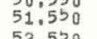 & $\begin{array}{l}0.99 \\
0.99\end{array}$ & $\begin{array}{l}713: 38875 \\
784: 38875\end{array}$ & $\frac{14}{20}>>C$ & 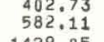 & $\begin{array}{l}20174 \\
20112\end{array}$ & $\begin{array}{l}1955 \\
19466 \\
\end{array}$ & & $\begin{array}{l}3086 \\
2978\end{array}$ & $\begin{array}{l}3086 \\
30278\end{array}$ & \\
\hline $\begin{array}{ll}0 \\
0270865 \\
0218865\end{array}$ & 50 & $\begin{array}{l}0.9898 \\
0.9892\end{array}$ & $\begin{array}{l}726.89175 \\
72955\end{array}$ & $\begin{array}{l}22.01 \\
23.97\end{array}$ & $\begin{array}{l}1429.05 \\
646.19\end{array}$ & $\begin{array}{l}21002 \\
20436 \\
20436\end{array}$ & $\begin{array}{ll}20043 \\
19752\end{array}$ & 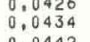 & $\begin{array}{l}2612 \\
2657\end{array}$ & 157 & \\
\hline 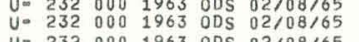 & $\begin{array}{l}54,550 \\
55,550\end{array}$ & $\begin{array}{l}0,9973 \\
0,9913\end{array}$ & $\begin{array}{l}700.48556 \\
675: 49255\end{array}$ & $\begin{array}{l}48.56 \\
19.41\end{array}$ & $\begin{array}{l}1285.32 \\
504: 40\end{array}$ & $\begin{array}{l}2071 \\
20451\end{array}$ & 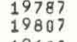 & $\begin{array}{l}0,042 \\
0,0450\end{array}$ & $\begin{array}{l}24244 \\
2255\end{array}$ & $\begin{array}{l}2484 \\
2255\end{array}-\mathrm{l}$ & \\
\hline 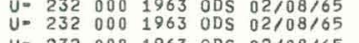 & 6.550 7,550 & $\begin{array}{l}0,989 \\
0: 9707\end{array}$ & $\begin{array}{l}6511,81368 \\
629,35841\end{array}$ & $\begin{array}{l}42,67 \\
65,92\end{array}$ & $\begin{array}{l}1008930 \\
1653: 80\end{array}$ & $\begin{array}{l}20452 \\
20012\end{array}$ & $\begin{array}{l}19606 \\
18975\end{array}$ & $\begin{array}{l}0.0458 \\
0,0466\end{array}$ & $\begin{array}{l}2385 \\
2480\end{array}$ & $\begin{array}{l}2385 \\
2480\end{array}$ & \\
\hline $\begin{array}{l}232 \\
232 \\
232000\end{array}$ & 9.550 & $\begin{array}{l}0,9878 \\
0,9896\end{array}$ & $\begin{array}{l}6088.04384 \\
587,79401\end{array}$ & $\begin{array}{l}27.20 \\
23.13\end{array}$ & $\begin{array}{l}670.66 \\
560.84\end{array}$ & $\begin{array}{l}20805 \\
19969\end{array}$ & $\begin{array}{l}20076 \\
19307\end{array}$ & $\begin{array}{l}0.044 \\
0.0482\end{array}$ & $\begin{array}{l}1576 \\
2162\end{array}$ & $\begin{array}{l}1576 \\
2162\end{array}$ & \\
\hline $\begin{array}{ll}20001963 \text { ODS } 02 / 08 / 65 \\
2\end{array}$ & 60,550 & 0,9882 & 568,53918 & 26.31 & 627.22 & 20095 & 19401 & 0490 & 1790 & 79 & \\
\hline 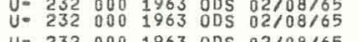 & 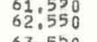 & $\begin{array}{l}0: 965 \\
0: 9852\end{array}$ & $\begin{array}{l}550: 21519 \\
532: 76301\end{array}$ & $\begin{array}{l}30,0 ; 1 \\
32,9\end{array}$ & $\begin{array}{l}760.28 \\
761: 06\end{array}$ & $\begin{array}{l}19903 \\
19891\end{array}-120$ & $\begin{array}{l}1918 \varepsilon \\
1914 \varepsilon\end{array}$ & 99 & 05 & 052 & \\
\hline 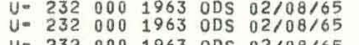 & $\begin{array}{l}63.5 \\
64.5\end{array}$ & $\begin{array}{l}0.9768 \\
0.9916\end{array}$ & $\begin{array}{l}516.12819 \\
500.26049\end{array}$ & $\begin{array}{l}52.13 \\
18.64\end{array}$ & $\begin{array}{l}1184.36 \\
416: 85\end{array}$ & $\begin{array}{l}19772 \\
19838 \\
1938-3\end{array}$ & $\begin{array}{l}18866 \\
19215\end{array}$ & $\begin{array}{l}\frac{15}{23} \\
23\end{array}$ & 9 & $\begin{array}{l}1574 \\
1229\end{array}$ & \\
\hline 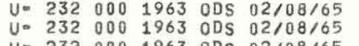 & $\begin{array}{l}65,550 \\
66,550\end{array}$ & $\begin{array}{l}0.965 \\
0.9870\end{array}$ & $\begin{array}{l}485.113444 \\
470.6406\end{array}$ & $\begin{array}{l}89.31 \\
28.99\end{array}$ & $\begin{array}{r}1967.17 \\
628.81\end{array}$ & $\begin{array}{l}20247 \\
19508\end{array}$ & $\begin{array}{l}18995 \\
18811\end{array}$ & $\begin{array}{l}0,005 \\
0,05\end{array}$ & $\begin{array}{l}1078 \\
1000\end{array}$ & $\begin{array}{l}1078 \\
1000\end{array}$ & \\
\hline $\begin{array}{l}02 / 08865 \\
02 / 08 / 65\end{array}$ & $\begin{array}{l}550 \\
550\end{array}$ & $\begin{array}{l}0,902 \\
0 ; 9699\end{array}$ & $\begin{array}{l}4566.81251 \\
443.58186\end{array}$ & $\begin{array}{l}44,32 \\
67,76\end{array}$ & $\begin{array}{r}9477: 29 \\
1427: 13\end{array}$ & $\begin{array}{l}19753 \\
19148\end{array}$ & $\begin{array}{l}18916 \\
18144\end{array}$ & 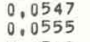 & $\begin{array}{l}739 \\
840\end{array}$ & $\begin{array}{l}739 \\
840\end{array}$ & \\
\hline 20001963 ODS $02 / 08 / 65$ & 0 & 0.9731 & 430.91779 & 60.4 & 1255.27 & 19177 & 18231 & 0.0563 & $0.7-2$ & 81 & \\
\hline 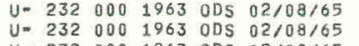 & .550 & $\begin{array}{l}1,0009 \\
0,9637\end{array}$ & $\begin{array}{l}418,78842 \\
407,16405\end{array}$ & $\begin{array}{l}-2,06 \\
81,94\end{array}$ & $\begin{array}{r}-42.18 \\
1153.49\end{array}$ & $\begin{array}{l}18024 \\
17783\end{array}$ & 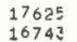 & 50571 & 930 & 930 & \\
\hline $\begin{array}{l}02 / 0 \\
02 / 0\end{array}$ & $\begin{array}{l}2.550 \\
3,550\end{array}-20$ & $\begin{array}{l}0.97 \\
0.98\end{array}$ & 385,3 & & & $\begin{array}{l}10089 \\
10018\end{array}$ & & & 692 & 692 & \\
\hline $02 / 08165$ & 4.550 & 0844 & 375.05366 & 34.91 & 676.15 & $\begin{array}{l}14924 \\
1924\end{array}$ & 14393 & 0.0 & 年 & 年 & \\
\hline $\begin{array}{l}1963 \text { ODS } 022 / 108 / 165 \\
1963 \text { ODS } 02 / 08 / 65\end{array}$ & $\begin{array}{l}75,550 \\
76,550\end{array}$ & $\begin{array}{l}0.9893 \\
1 ; 0045\end{array}$ & $\begin{array}{l}365.190074 \\
355,7183\end{array}$ & $\begin{array}{l}23,78 \\
-9,87\end{array}$ & $\begin{array}{l}\begin{array}{r}454.52 \\
-186.19\end{array} \\
\end{array}$ & $\begin{array}{l}12260 \\
{ }_{8388}\end{array}$ & 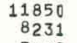 & 0.0 & $\begin{array}{l}591 \\
715\end{array}$ & 勇151 & \\
\hline $\begin{array}{l}23 \\
23 \\
23\end{array}$ & $\begin{array}{l}78: 5 \\
79.5\end{array}$ & $\begin{array}{l}0.96 \\
0.98 \\
0.98\end{array}$ & $\begin{array}{l}336,82853 \\
337.82853\end{array}$ & 29.58 & 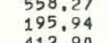 & $\begin{array}{l}5363 \\
5120 \\
7720\end{array}$ & 51 & & 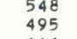 & s & \\
\hline 65 & & & 321.2 & & & 11575 & & & & & \\
\hline $\begin{array}{l}02 / 08 / 65 \\
02 / 08 / 65\end{array}$ & .550 $.500-300$ & $\begin{array}{l}0.987 \\
0: 9859\end{array}$ & $\begin{array}{l}315 ; 43015 \\
305: 88244\end{array}$ & $\begin{array}{l}70,40 \\
33,39\end{array}$ & $\begin{array}{l}1246 ; 36 \\
58 ; 98\end{array}$ & $\begin{array}{l}211 \\
1621\end{array}$ & $\begin{array}{l}13420 \\
15617 \\
150\end{array}$ & $\begin{array}{l}0.0661 \\
0.069\end{array}$ & 年 & 402 & \\
\hline 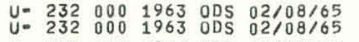 & $\begin{array}{l}3.550 \\
4.590\end{array}$ & $\begin{array}{l}0: 917 \\
0: 9928\end{array}$ & $\begin{array}{l}299860411 \\
291 ; 58251\end{array}$ & $\begin{array}{l}63.7 \\
16.1\end{array}$ & $\begin{array}{ll}11011 \\
274: 96\end{array}$ & $\begin{array}{l}17026 \\
17377\end{array}$ & $\begin{array}{l}161635 \\
16853\end{array}$ & $0: 00$ & $\begin{array}{l}843 \\
684\end{array}$ & $\begin{array}{l}084 \\
684\end{array}$ & \\
\hline 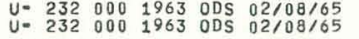 & $\begin{array}{l}5.550 \\
6.550\end{array}$ & $\begin{array}{l}0,9957 \\
0,9883\end{array}$ & $\begin{array}{l}284: 80569 \\
277: 26242\end{array}$ & $\begin{array}{c}96,2 \\
26,0\end{array}$ & 161: & $\begin{array}{l}183 \\
184,\end{array}$ & $\begin{array}{l}1700 \\
17782\end{array}$ & 0 & 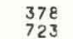 & 723 & \\
\hline 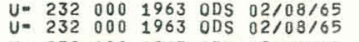 & .5 & $\begin{array}{l}0.9980 \\
0: 9650\end{array}$ & $\begin{array}{l}277.99207 \\
265 ; 83463\end{array}$ & $78: 9^{4}$ & 1287 & $\begin{array}{l}187 \\
189\end{array}$ & . & & $\begin{array}{l}613 \\
801\end{array}$ & $\begin{array}{l}613 \\
801\end{array}$ & \\
\hline 2320001963 ODS $02 / 08 / 65$ & 8 & 0,98 & 259.93066 & 34.80 & 561.0 & 18582 & 17871 & 0.0725 & 942 & & \\
\hline 65 & .5 & $\begin{array}{l}0.96 \\
0.99\end{array}-19$ & $\begin{array}{l}254.2 \\
248: 6\end{array}-6$ & $\begin{array}{l}83.81 \\
17.25\end{array}-1$ & $\begin{array}{l}1336 . \\
272 .\end{array}$ & 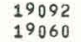 & $\begin{array}{l}17960 \\
1847 t\end{array}-10$ & $\begin{array}{l}.0733 \\
.0742\end{array}$ & $\begin{array}{l}705 \\
730\end{array}$ & 35 & \\
\hline $\begin{array}{l}02 / 108 / 65 \\
02 / 08 / 65\end{array}$ & 0 & $\begin{array}{l}0,9987 \\
0,9875\end{array}$ & 238.177 & $\begin{array}{l}47.67 \\
27.94\end{array}$ & $\begin{array}{l}743 \\
431 \\
4\end{array}$ & $\begin{array}{l}19439 \\
19128\end{array}$ & 18453 & & 76 & & \\
\hline $\begin{array}{l}8 / 65 \\
8 / 65\end{array}$ & $\begin{array}{l}4.550 \\
. .550\end{array}-50$ & $\begin{array}{l}0.99 \\
0.98\end{array}-19$ & $\begin{array}{l}233.16022 \\
228.31125 \\
2.325\end{array}$ & 30,77 & $\begin{array}{l}245 \\
464 \\
464\end{array}$ & $\begin{array}{l}18787 \\
18875\end{array}-\mathrm{l}$ & ${ }_{181}^{182}$ & & 780 & 780 & \\
\hline $02 / 0$ & & & $\begin{array}{l}8.00335 \\
.04540\end{array}$ & & & & & & & & \\
\hline 000196300 & & 1. & 33240 & & & & & & & & \\
\hline 0001903 ODS & & 1,0089 & 法 & & & & & & & & \\
\hline
\end{tabular}




\begin{tabular}{|c|c|c|c|c|c|c|c|c|c|c|c|}
\hline IDENTIFICATION & DELAY & TRANS, & ENERGY & $x-s \in c$ & $\begin{array}{l}x=S E C \\
R=00 T E\end{array}$ & OPEN & SAMI & $\begin{array}{l}\text { FRAC, } \\
\text { OFA OCT }\end{array}$ & $\begin{array}{c}\text { FAST } \\
\text { OPEN }\end{array}$ & S $_{\text {MPL }}$ & PYS PSS \\
\hline $\begin{array}{ll}u=2 \\
y=2 \\
a\end{array}$ & $\begin{array}{l}100.550 \\
101.520\end{array}$ & $\begin{array}{l}1,0114 \\
0.9806\end{array}$ & $\begin{array}{l}206,16956 \\
202,12910\end{array}$ & $\begin{array}{r}-25.09 \\
43.50\end{array}$ & $\begin{array}{l}-360.31 \\
-3618\end{array}$ & $\begin{array}{l}18982 \\
19959\end{array}$ & $\begin{array}{l}51876 \\
1857\end{array}$ & 0.0814 & 60 & 688 & \\
\hline 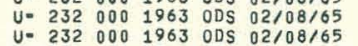 & $\begin{array}{l}102,550 \\
103,550\end{array}$ & 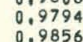 & $\begin{array}{ll}208 \\
198,20626 \\
1949652\end{array}$ & 46. & $\begin{array}{l}649.73 \\
648 \\
448\end{array}$ & 19105 & $\begin{array}{l}1828 \\
18280\end{array}-135$ & 0.0831 & $\begin{array}{l}820 \\
658 \\
625\end{array}$ & $\begin{array}{lll}620 \\
658 \\
625\end{array}$ & \\
\hline 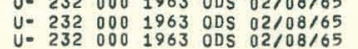 & $\begin{array}{l}104350 \\
104550 \\
105\end{array}$ & 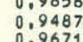 & $\begin{array}{l}194.09652 \\
1906558\end{array}$ & 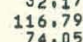 & $\begin{array}{l}461.51 \\
1612.83 \\
1012.61\end{array}$ & $\begin{array}{r}18834 \\
19280\end{array}$ & $\begin{array}{l}17785 \\
71725\end{array}$ & $\begin{array}{l}0.0839 \\
0.0847\end{array}-0845-3$ & $\begin{array}{l}625 \\
544\end{array}$ & $\begin{array}{l}625 \\
544\end{array}$ & \\
\hline & & & & & & & & & 577 & & \\
\hline 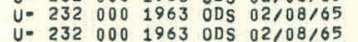 & 107.550 & 0.9915 & $\begin{array}{l}80.20543 \\
0150049\end{array}$ & 18.87 & $\begin{array}{l}253.31 \\
446.61\end{array}-100$ & 19429 & 18820 & 0.0871 & 608 & 608 & \\
\hline 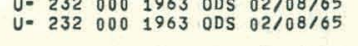 & $\begin{array}{l}108.550 \\
109.550\end{array}$ & 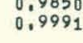 & $\begin{array}{l}176,90049 \\
173,68565\end{array}$ & 2,00 & 26.31 & 19056 & 18600 & 0,0887 & 746 & 746 & \\
\hline 2320001963 ODS $02 / 08 / 65$ & 110.550 & 1.0089 & 170,55765 & -19.73 & -257.64 & 18939 & 18668 & 0,0895 & 564 & 564 & \\
\hline 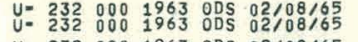 & $\begin{array}{l}\frac{111}{112}: 5500 \\
550\end{array}$ & $\begin{array}{l}0: 9807 \\
0: 9825\end{array}-250$ & $\begin{array}{l}167,51340 \\
164.54993\end{array}$ & $\begin{array}{l}44325 \\
39\end{array} 25$ & $\begin{array}{l}5592789 \\
503: 49\end{array}-20$ & $\begin{array}{l}18864 \\
18640\end{array}$ & $\begin{array}{l}18169 \\
17891\end{array}$ & $\begin{array}{l}0: 0904 \\
0: 0912\end{array}-12-100$ & 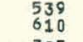 & $\begin{array}{l}539 \\
610\end{array}$ & \\
\hline 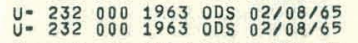 & $\begin{array}{l}113.550 \\
114: 550\end{array}$ & 0,9782 & $\begin{array}{l}161: 06441 \\
158: 85413\end{array}$ & $\begin{array}{l}488,88 \\
80.62\end{array}$ & $\begin{array}{l}621.55 \\
1016: 17\end{array}$ & $\begin{array}{l}18648 \\
19050\end{array}$ & $\begin{array}{l}17281 \\
17946\end{array}$ & $\begin{array}{l}0,0920 \\
0: 0928\end{array}-10$ & $\begin{array}{l}705 \\
540\end{array}$ & $\begin{array}{l}775 \\
540\end{array}$ & \\
\hline 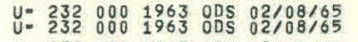 & $\begin{array}{l}115: 550 \\
116: 5 P_{0}\end{array}$ & $0: 97705$ & $\begin{array}{l}156: 11650 \\
153,44903\end{array}$ & 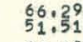 & $\begin{array}{l}828.32 \\
638 ; 09\end{array}$ & $\begin{array}{l}18971 \\
19251\end{array}$ & $\begin{array}{l}17889 \\
18378\end{array}$ & $\begin{array}{l}0: 0936 \\
0: 0944\end{array}-14$ & $\begin{array}{l}4999 \\
415\end{array}$ & $\begin{array}{l}499 \\
415\end{array}$ & \\
\hline 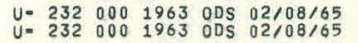 & $\begin{array}{l}1178,5500 \\
118: 550\end{array}$ & $0: 9712$ & $\begin{array}{l}150,84934 \\
148,31517\end{array}$ & $\begin{array}{l}64.68 \\
63.29 \\
63.29\end{array}$ & $794: 37$ & $\begin{array}{l}18873 \\
18581\end{array}$ & $\begin{array}{l}17907 \\
17641\end{array}$ & $\begin{array}{l}0,0952 \\
0,0960\end{array}-3$ & $\begin{array}{l}631 \\
656 \\
656-25 l\end{array}$ & $\begin{array}{l}6511 \\
656\end{array}$ & \\
\hline$U=2320001963$ ODS $02108 / 65$ & 119,550 & 0.9618 & 145,84432 & 86,46 & 1044,18 & 18613 & & 0,0968 & 383 & 383 & \\
\hline DS $02 / 08 / 65$ & 120,520 & .9262 & 143.43471 & 170.05 & 2036.58 & 18360 & 16512 & .0976 & 354 & 年 & \\
\hline 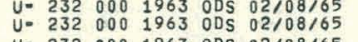 & $\begin{array}{l}121.550 \\
122,550\end{array}$ & $\begin{array}{l}0,9317 \\
0,9810\end{array}-20$ & $\begin{array}{l}141.08433 \\
138,79124\end{array}$ & $\begin{array}{r}1556.94 \\
42.52\end{array}$ & 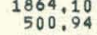 & $\begin{array}{l}17976 \\
17089\end{array}$ & $\begin{array}{l}166361 \\
1637 \varepsilon\end{array}$ & $\begin{array}{l}0: 0985 \\
0,0993\end{array}$ & $\begin{array}{c}507 \\
508\end{array}-1-30$ & $\begin{array}{l}557 \\
508\end{array}$ & \\
\hline 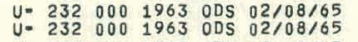 & $\begin{array}{l}123,550 \\
124: 550\end{array}$ & $\begin{array}{l}0,9808 \\
1,0007\end{array}$ & $\begin{array}{l}136,55361 \\
134,36966\end{array}$ & $\begin{array}{l}43,04 \\
-1,45\end{array}$ & $\begin{array}{r}5022.95 \\
-16: 75\end{array}$ & $\begin{array}{l}16651 \\
15223\end{array}$ & $\begin{array}{l}15554 \\
14882\end{array}$ & $\begin{array}{l}0,1001 \\
0,1009\end{array}$ & $\begin{array}{l}270 \\
460\end{array}$ & $\begin{array}{l}270 \\
460\end{array}$ & \\
\hline 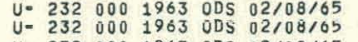 & $\begin{array}{l}125,520 \\
126.520\end{array}$ & $\begin{array}{l}0,9498 \\
0,8870\end{array}-100$ & $\begin{array}{l}132,23769 \\
130.15606\end{array}$ & $\begin{array}{l}114424 \\
265 ; 82\end{array}-120$ & $\begin{array}{l}13133.65 \\
3032.63 \\
303\end{array}$ & $\begin{array}{l}14598 \\
15752\end{array}$ & $\begin{array}{l}13565 \\
13650\end{array}$ & $\begin{array}{l}0.1017 \\
0,1025\end{array}$ & $\begin{array}{l}543 \\
383\end{array}$ & $\begin{array}{l}543 \\
383\end{array}$ & \\
\hline $\begin{array}{l}S 02 / 58 / 65 \\
s 02 / 08 / 65\end{array}$ & $\begin{array}{l}127.550 \\
128,550\end{array}$ & $\begin{array}{l}0,9482 \\
0.9739\end{array}-19$ & $\begin{array}{l}128,12320 \\
126,13759\end{array}$ & $\begin{array}{r}117,93 \\
58,69\end{array}$ & $\begin{aligned} 1334488 \\
659: 10\end{aligned}$ & 304 & $\begin{array}{l}15092 \mathrm{z} \\
1646 ?\end{array}$ & & $\begin{array}{l}466 \\
427 \\
427\end{array}-30$ & $\begin{array}{l}466 \\
427\end{array}$ & \\
\hline$u=2320001963$ ODS $02108 / 65$ & 129.550 & 0.9723 & $\begin{array}{ll} & \end{array}$ & 62.24 & $\begin{array}{l}693.102 \\
693\end{array}$ & 17788 & 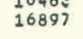 & 0.1049 & 421 & 421 & \\
\hline $502 / 08 / 65$ & 130.550 & 0,9828 & 122,30239 & 38.47 & 425.42 & 16818 & 147 & 057 & 386 & 386 & \\
\hline 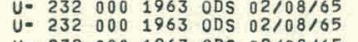 & $\begin{array}{l}131.550 \\
132.550\end{array}$ & 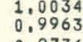 & $\begin{array}{l}\begin{array}{l}120.45005 \\
118.63948\end{array} \\
10396\end{array}$ & & $\begin{array}{l}-81.59 \\
88.67\end{array}$ & $\begin{array}{l}16330 \\
117768\end{array}$ & 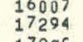 & $\begin{array}{l}0.1066 \\
0.1074\end{array}-\mathrm{r}-\mathrm{r}$ & $\begin{array}{l}404 \\
464 \\
464\end{array}$ & 464 & \\
\hline 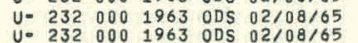 & $\begin{array}{l}133.550 \\
134.520\end{array}$ & $\begin{array}{l}0,9736 \\
0,9615\end{array}$ & $\begin{array}{l}116,86942 \\
115,13869\end{array}$ & $\begin{array}{l}59,38 \\
87.10\end{array}$ & $\begin{array}{l}641.95 \\
0344\end{array}$ & $\begin{array}{l}18830 \\
1894\end{array}$ & $\begin{array}{l}17909 \\
17797\end{array}$ & 1008 & $\begin{array}{l}366 \\
257\end{array}$ & $\begin{array}{l}366 \\
257\end{array}$ & \\
\hline $\begin{array}{llll}232 & 000 & 1963 & 0 \\
232 & 000 & 1963 & 0\end{array}$ & $\begin{array}{l}135.550 \\
136.550\end{array}$ & 0.9601 & $\begin{array}{l}113.44612 \\
111.79060\end{array}$ & $\begin{array}{l}90.32 \\
80.63\end{array}-12$ & $\begin{array}{l}962.00 \\
852,47\end{array}$ & $\begin{array}{l}18820 \\
18911\end{array}$ & $\begin{array}{l}17651 \\
17814\end{array}$ & $\begin{array}{l}0,1098 \\
0.1106\end{array}$ & $\begin{array}{l}382 \\
437\end{array}$ & $\begin{array}{l}382 \\
437\end{array}$ & \\
\hline 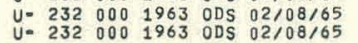 & $\begin{array}{l}137.550 \\
1385550\end{array}$ & $\begin{array}{l}0,79254 \\
0,8766\end{array}$ & $\begin{array}{l}110.17105 \\
108,58645\end{array}$ & $\begin{array}{l}\frac{171}{29}: 92 \\
21: 93\end{array}$ & $\begin{array}{l}\frac{1804}{3042: 46}-10 \\
3040\end{array}$ & $\begin{array}{l}18534 \\
18507\end{array}$ & $\begin{array}{l}16756 \\
11599\end{array}$ & $\begin{array}{l}0,1114 \\
0,1122\end{array}$ & $\begin{array}{l}460 \\
419\end{array}$ & $\begin{array}{l}460 \\
419\end{array}$ & \\
\hline U. 2320001963 OUS $02 / 08 / 65$ & 139,550 & 0.9219 & 107.03579 & 180.33 & 1865,68 & 3453 & 16615 & 0,1130 & 470 & 470 & \\
\hline $\begin{array}{l}02 / 08 / 65 \\
02 / 0865\end{array}$ & $\begin{array}{l}140.550 \\
141\end{array}$ & 0.9582 & 105.5 & & 75 & 95 & 500 & & 470 & 70 & \\
\hline $\begin{array}{l}02108 / 165 \\
02 / 65\end{array}$ & $\begin{array}{l}141,500 \\
142,550\end{array}$ & 0.9803 & $\begin{array}{l}104.05286 \\
102,57801\end{array}$ & $\begin{array}{l}75: \\
44:\end{array}$ & $\begin{array}{l}773.69 \\
445.62\end{array}$ & 567 & 792 & 75 & $\begin{array}{l}413 \\
456\end{array}$ & 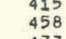 & \\
\hline 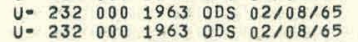 & $\begin{array}{l}143.550 \\
144.550\end{array}$ & $\begin{array}{l}0,9974 \\
0,9822\end{array}-12$ & $\begin{array}{l}101.15382 \\
99.75910\end{array}$ & $\begin{array}{r}5,86 \\
39,77\end{array}$ & $\begin{array}{r}58,94 \\
397,17\end{array}$ & $\begin{array}{l}18214 \\
18392\end{array}-V 0$ & $1764 \varepsilon$ & 0.1171 & 412 & 4: & \\
\hline 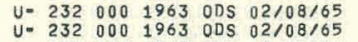 & $\begin{array}{l}145.550 \\
146.550\end{array}$ & 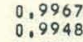 & $\begin{array}{l}98,3930202 \\
9705481\end{array}$ & $\begin{array}{r}7,24 \\
11.64\end{array}$ & $\begin{array}{r}71,82 \\
114: 68\end{array}$ & $\begin{array}{l}18453 \\
18432\end{array}$ & $\begin{array}{l}17966 \\
17918\end{array}$ & $\begin{array}{c}1179 \\
1187\end{array}$ & 列 534 & $\begin{array}{l}534 \\
485\end{array}$ & \\
\hline $\begin{array}{llll}232 & 2000 & 1963 \\
232 & 000 & 1963\end{array}$ & $\begin{array}{l}147.550 \\
148,550\end{array}$ & $\begin{array}{l}0,0948 \\
1,0033\end{array}-100$ & $\begin{array}{l}95,74371 \\
94,45901\end{array}$ & $\begin{array}{l}11.50 \\
-7.31\end{array}-31$ & $\begin{array}{l}112,51 \\
-71,03 \\
-17\end{array}$ & $\begin{array}{l}3686 \\
3501\end{array}$ & 134 & 1203 & $\begin{array}{l}409 \\
541\end{array}$ & $\begin{array}{l}409 \\
541\end{array}$ & \\
\hline DS $02 / 08 / 65$ & 149.550 & 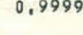 & 93,19999 & 0.31 & 2,98 & & 860 & & 412 & 2 & \\
\hline 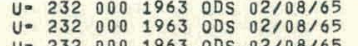 & $\begin{array}{l}150.550 \\
151.520\end{array}$ & $\begin{array}{l}0.9890 \\
0.9898\end{array}-120$ & $\begin{array}{l}91.96597 \\
90,7531\end{array}$ & $\begin{array}{l}24.59 \\
22.80\end{array}-30$ & $\begin{array}{l}5.80 \\
: 17\end{array}$ & $\begin{array}{l}676 \\
6320\end{array}$ & $\begin{array}{l}2 \varepsilon \\
1,3 \\
1.3\end{array}$ & 11228 & $\begin{array}{l}490 \\
555\end{array}$ & 年 & \\
\hline 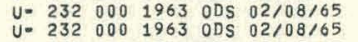 & $\begin{array}{l}152,550 \\
153,550\end{array}$ & $\begin{array}{l}0,9716 \\
0,9929\end{array}-19$ & $\begin{array}{l}89.57035 \\
88: 40749\end{array}$ & $\begin{array}{l}63.94 \\
15,82\end{array}-320$ & $\begin{array}{l}605 \\
148: 12 \\
67\end{array}$ & $\begin{array}{lll}1 & 17021 \\
1\end{array}$ & ${ }_{16}^{16}>-1$ & 0.1244 & 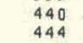 & 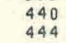 & \\
\hline 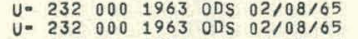 & $\begin{array}{l}154.520 \\
155.550\end{array}$ & $\begin{array}{l}0,9961 \\
0,9871\end{array}$ & $\begin{array}{l}87.26773 \\
86.1469\end{array}$ & $\begin{array}{r}88.70 \\
28.75\end{array}$ & $\begin{array}{r}81.29 \\
266: 80\end{array}$ & $\begin{array}{l}17966 \\
17766\end{array}$ & $\begin{array}{l}1748 z \\
1713 z\end{array}$ & 60 & $\begin{array}{l}605 \\
671\end{array}$ & $\begin{array}{l}605 \\
671\end{array}$ & \\
\hline 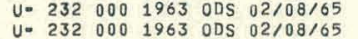 & $\begin{array}{r}156.550 \\
157.520\end{array}$ & 1.0 & $\begin{array}{l}85.05 \\
83.9\end{array}$ & 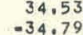 & $\begin{array}{l}318.41 \\
318.79\end{array}$ & & & & & & \\
\hline 02108 & 158.550 & 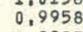 & 82.91941 & 9.22 & 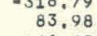 & 68 & 17848 & 84 & 540 & $\begin{array}{ll}540 & 0\end{array}$ & \\
\hline 0210 & 159,5 & 0.99 & 81,8 & 16 & 12 & 8809 & & 92 & 518 & & \\
\hline $\begin{array}{l}0001963 \text { ODS } 02 / 08 / 65 \\
000 \quad 1963 \text { DOS } 02 / 08 / 65 \\
000\end{array}$ & $\begin{array}{l}160.550 \\
161.550\end{array}$ & 0.9922 & 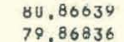 & 17.3 & $\begin{array}{l}6.34 \\
7,07\end{array}$ & $\begin{array}{l}18685 \\
1058\end{array}$ & 11 & & 56 & 56 & \\
\hline $\begin{array}{l}1278765 \\
02108 / 55\end{array}$ & $\begin{array}{l}101,520 \\
162,520\end{array}$ & 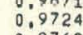 & 78.88 & 6 & & $\begin{array}{ll}18584 \\
18502\end{array}$ & & & 744 & & \\
\hline 1963 ODS $02 / 08 / 65$ & $\begin{array}{l}163,550 \\
164,550\end{array}$ & 0,9909 & $76: 98266$ & 30.60 360 & 88.66 & $\begin{array}{l}1072 \\
18297\end{array}$ & & & 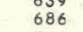 & 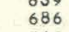 & \\
\hline 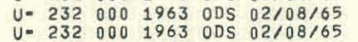 & $\begin{array}{l}165.550 \\
166,550\end{array}$ & $\begin{array}{l}0,8688 \\
0.7550\end{array}$ & $\begin{array}{l}76.05555 \\
75.1488\end{array}$ & $\begin{array}{l}311,92 \\
622,99\end{array}$ & $\begin{array}{l}2720.28 \\
540044\end{array}$ & $\begin{array}{l}1857 \\
1888\end{array}$ & $\begin{array}{l}1575 \varepsilon \\
1357\end{array}$ & 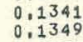 & $\begin{array}{l}766 \\
7611 \\
81\end{array}$ & $\begin{array}{l}766 \\
811\end{array}$ & \\
\hline $\begin{array}{l}02 / 0865 \\
02 / 108 / 65\end{array}$ & $\begin{array}{l}167.550 \\
168.550\end{array}$ & $\begin{array}{l}0,8047 \\
0,9232\end{array}-120$ & $\begin{array}{l}74,25058 \\
73 ; 37214\end{array}$ & $\begin{array}{l}481,79 \\
177: 17\end{array}$ & $\begin{array}{l}\begin{array}{l}4151,55 \\
151 ; 760\end{array} \\
100\end{array}$ & $\begin{array}{l}187 \\
186\end{array}$ & $\begin{array}{l}147 \\
168\end{array}$ & & 766 & $\frac{8}{7}$ & \\
\hline$U=232000 \quad 1963 \quad$ ODS $02 / 08 / 65$ & 169,550 & 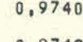 & 72,50920 & 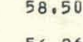 & & & & & 784 & & \\
\hline 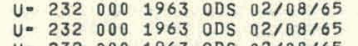 & $\begin{array}{l}170.550 \\
171.550\end{array}$ & $\begin{array}{l}0,9749 \\
0,9841\end{array}-120$ & $\begin{array}{l}71.66139 \\
70.82837\end{array}$ & $\begin{array}{l}56.26 \\
35.59\end{array}$ & $\begin{array}{l}7.27 \\
69.56\end{array}$ & 1707 & 178 & & $\begin{array}{l}60 \\
21\end{array}$ & 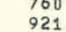 & \\
\hline & $\begin{array}{l}172.550 \\
173550\end{array}$ & & & & & & & & & & \\
\hline $\begin{array}{lll}1963 \text { ODS } 02 / 0 & \end{array}$ & $\begin{array}{l}1735.550 \\
17550\end{array}$ & $\begin{array}{l}0.9969 \\
0.9962\end{array}$ & $\begin{array}{l}68.41463 \\
68.203\end{array}$ & $\because:$ & 16 & $\begin{array}{l}18019 \\
18018\end{array}$ & & & 800 & 8 & \\
\hline 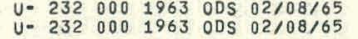 & $\begin{array}{l}175.550 \\
176,550\end{array}$ & $\begin{array}{l}0,9984 \\
1\end{array}$ & $\begin{array}{l}67.63742 \\
68.87338\end{array}$ & 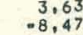 & $\begin{array}{r}29,85 \\
-69 ; 30\end{array}-30-100$ & $\begin{array}{l}17082 \\
16797\end{array}-30$ & $\begin{array}{l}16661 \\
1647 \%\end{array}$ & & 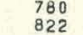 & 82 & \\
\hline & t7 & & & & & & & & & & \\
\hline 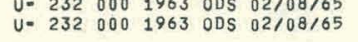 & $\begin{array}{l}178,550 \\
179,550\end{array}$ & $\begin{array}{l}0,9875 \\
0,9903\end{array}$ & $\begin{array}{l}65.38362 \\
64.65735\end{array}$ & $\begin{array}{l}27,96 \\
21.68\end{array}$ & 29 & $\begin{array}{l}18399 \\
18090\end{array}$ & 501 & & 790 & 90 & \\
\hline 1963 ODS 02 & 180.5 & 1.0 & & & & & & & a & 型 & \\
\hline & ${ }_{182}^{181}$ & & $\begin{array}{l}63 ; 2,2 \\
62,5\end{array}$ & & $?^{0}$ & 16 & & & & $\begin{array}{l}7.5 \\
68\end{array}$ & \\
\hline $\begin{array}{l}02 / 0 \\
02 / 0\end{array}$ & $\begin{array}{l}183.550 \\
184.550\end{array}$ & $\begin{array}{l}1: 053 \\
0 ; 9915\end{array}$ & $\begin{array}{l}61,86997 \\
61 ; 20129\end{array}$ & $\begin{array}{r}-11: 65 \\
18: 95\end{array}$ & $\begin{array}{l}-91,63 \\
148,24\end{array}$ & $\begin{array}{l}18175 \\
18130\end{array}$ & $\begin{array}{l}178 \\
175\end{array}$ & & $\begin{array}{l}687 \\
630\end{array}$ & $\begin{array}{l}687 \\
630\end{array}$ & \\
\hline 1963 ODS $02 / 08 / 65$ & $\begin{array}{l}185.550 \\
186.550\end{array}$ & 0.9 & $\begin{array}{l}60.54340 \\
59: 89605\end{array}$ & $\begin{array}{l}20.87 \\
66494\end{array}$ & $\begin{array}{l}162.38 \\
502.58\end{array}$ & $\begin{array}{l}18135 \\
18339\end{array}$ & $\frac{175}{173}$ & & $\begin{array}{l}688 \\
645\end{array}$ & $\begin{array}{l}6 . \\
6 \\
6\end{array}$ & \\
\hline & & & $\begin{array}{l}59.2 \\
58.6\end{array}$ & & & & & & 6.2 & & \\
\hline $232000196300502 / 08 / 6$ & 189,55 & 0,989 & 58,0151 & 24. & & 8 & 17 & & 497 & 497 & \\
\hline $\begin{array}{l}02 / 08 / 65 \\
02 / 18865\end{array}$ & $\begin{array}{l}190,550 \\
191.550\end{array}$ & 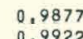 & $\begin{array}{l}57.40778 \\
56.8095\end{array}$ & 27. & & $\begin{array}{l}18037 \\
18143\end{array}$ & $\begin{array}{l}17403 \\
17585\end{array}$ & & 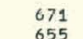 & 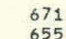 & \\
\hline & 192 & 0 & $\begin{array}{l}56.22140 \\
55 \% 641155\end{array}$ & 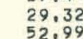 & & 146 & & & 566 & 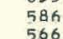 & \\
\hline 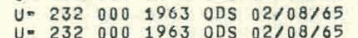 & 194. & & 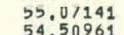 & 然 & & $8029-2-3-3$ & & & 677 & $\begin{array}{l}65 \\
67 \\
67\end{array}$ & \\
\hline & $196.550 \mathrm{C} C \mathrm{C}$ - $\mathrm{C}$ & & & & & & & & & & \\
\hline 年 & 198, & & & & & & & & 711 & & \\
\hline & & & & 64.66 & & & & & í. & & \\
\hline
\end{tabular}




\begin{tabular}{|c|c|c|c|c|c|c|c|c|c|c|c|}
\hline IDENTIFICATION & DELA* & TRANS. & ENERGY & $x-s \varepsilon c$ & $X=S E C$ & OPEN COU & & $\begin{array}{l}\text { FRAC, } \\
\text { OF OCT }\end{array}$ & $\begin{array}{l}\text { FAST } \\
\text { OPEN }\end{array}$ & GSS & $\begin{array}{l}\text { No, PTS } \\
\text { AVE, }\end{array}$ \\
\hline $\begin{array}{l}\text { DS } \\
\text { DS } 02 / 08 / 165 \\
02 / 08 / 65\end{array}$ & $\begin{array}{l}200,550 \\
201.550\end{array}$ & $\begin{array}{l}0,9838 \\
1,0069\end{array}$ & $\begin{array}{l}51.82548 \\
51.31249\end{array}$ & $\begin{array}{r}36,27 \\
-15.18\end{array}$ & $\begin{array}{l}2601,10 \\
-108,71\end{array}$ & $\begin{array}{l}18148 \\
17861\end{array}$ & $\begin{array}{l}\text { la } \\
17441 \\
17568\end{array}$ & $\begin{array}{l}0.1624 \\
0,1633\end{array}$ & $\begin{array}{r}546 \\
580 \\
580\end{array}$ & $\begin{aligned} 546 \\
580\end{aligned}$ & \\
\hline 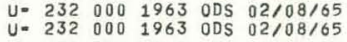 & $\begin{array}{l}202,530 \\
203.550\end{array}$ & $\begin{array}{l}0,9897 \\
1: 0142\end{array}-100$ & $\begin{array}{l}50.80708 \\
50: 30909\end{array}$ & $\begin{aligned} 22,94 \\
-31,34\end{aligned}$ & $\begin{array}{l}.163,51 \\
.222,26\end{array}$ & $\begin{array}{l}17990 \\
17863\end{array}$ & 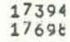 & $\begin{array}{ll}0 & 0,1641 \\
0 ; 1649 & \end{array}$ & $\begin{array}{l}543 \\
486\end{array}$ & $\begin{array}{l}543 \\
486\end{array}$ & \\
\hline 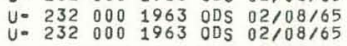 & $\begin{array}{l}204,550 \\
205,550\end{array}$ & $\begin{array}{l}0.9946 \\
0.9878\end{array}-130$ & $\begin{array}{l}49881399 \\
44: 34384\end{array}$ & $\begin{array}{l}\frac{12}{22} ; 04 \\
27.21\end{array}$ & $\begin{aligned} 84.98 \\
191: 11\end{aligned}$ & $\begin{array}{l}17772 \\
17744\end{array}$ & $\begin{array}{l}17365 \\
17126\end{array}$ & $\begin{array}{l}0: 1657 \\
0: 1665\end{array}$ & $\begin{array}{l}548 \\
561\end{array}$ & $\begin{array}{l}548 \\
561\end{array}$ & \\
\hline 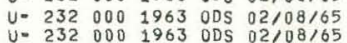 & $\begin{array}{l}206.550 \\
207550\end{array}$ & 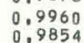 & $\begin{array}{l}488.85829 \\
448862\end{array}$ & $\begin{array}{r}8.86 \\
32.57\end{array}$ & $\begin{array}{l}61.92 \\
226.56\end{array}$ & $\begin{array}{l}17788 \\
11768\end{array}$ & $\begin{array}{l}17307 \\
17104\end{array}$ & $\begin{array}{l}0.1673 \\
0 ; 161\end{array}$ & $\begin{array}{l}489 \\
495\end{array}$ & $\begin{array}{l}489 \\
495\end{array}$ & \\
\hline 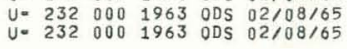 & $\begin{array}{l}208,550 \\
209,550\end{array}$ & $\begin{array}{l}0.9729 \\
0.9642\end{array}$ & $\begin{array}{l}47 \% 92568 \\
47: 46936\end{array}$ & $\begin{array}{l}61,02 \\
80,86\end{array}$ & $\begin{array}{l}422,45 \\
527,13\end{array}$ & $\begin{array}{l}17741 \\
117972\end{array}$ & $\begin{array}{l}16860 \\
16928 \\
1692-30\end{array}$ & $\begin{array}{l}0.1689 \\
0,1697\end{array}$ & $\begin{array}{l}550 \\
548\end{array}$ & $\begin{array}{l}550 \\
548\end{array}$ & \\
\hline 20001963 ODS $02 / 08 / 65$ & 210.550 & 0.9705 & 47.01952 & 66.38 & 455.18 & 17946 & 17014 & 0.1705 & 415 & 415 & \\
\hline $\begin{array}{l}\text { ODS } 022 / 108 / 65 \\
\text { ODS } 02 / 08 / 65\end{array}$ & $\begin{array}{l}211.550 \\
212.550\end{array}$ & $\begin{array}{l}0,9947 \\
0,9909\end{array}-99$ & $\begin{array}{l}46.57065 \\
46.13882\end{array}$ & $\begin{array}{l}11,88 \\
20,34\end{array}$ & $\begin{array}{r}81.10 \\
138,18\end{array}$ & $\begin{array}{l}17647 \\
17756\end{array}$ & 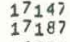 & $\begin{array}{l}0,1714 \\
0 ; 1722\end{array}$ & $\begin{array}{l}3833 \\
392\end{array}$ & $\begin{array}{l}383 \\
392 \\
392\end{array}$ & \\
\hline 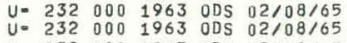 & $\begin{array}{l}213.550 \\
214: 550\end{array}$ & $\begin{array}{l}0,9838 \\
1,0063\end{array}-100$ & $\begin{array}{l}455,70772 \\
45.28263\end{array}$ & $\begin{aligned} 36.31 \\
-13 ; 87\end{aligned}$ & $\begin{array}{r}245.49 \\
-93: 31\end{array}$ & $\begin{array}{l}17751 \\
17092\end{array}$ & $\begin{array}{l}16771 \\
16801\end{array}$ & $\begin{array}{l}0: 1730 \\
0: 1738\end{array}$ & $\begin{array}{l}467 \\
387\end{array}$ & $\begin{array}{l}467 \\
387\end{array}$ & \\
\hline 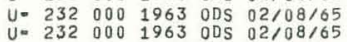 & $\begin{array}{l}215.550 \\
216.550\end{array}$ & $\begin{array}{l}0.9924 \\
0.9677\end{array}-170$ & $\begin{array}{l}44.86345 \\
44: 45006\end{array}$ & $\begin{array}{l}16 ; 91 \\
72 ; 82\end{array}$ & $\begin{array}{l}113.26 \\
485.52\end{array}$ & $\begin{array}{l}17132 \\
17408\end{array}$ & 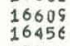 & $\begin{array}{l}0: 1746 \\
0: 1754\end{array}$ & $\begin{array}{l}332 \\
435\end{array}$ & $\begin{array}{l}332 \\
335 \\
435\end{array}$ & \\
\hline $\begin{array}{lllll}32 & 000 & 1963 & 005 & 02 / 08 / 65 \\
32 & 000 & 1963 & 005 & 02 / 0865\end{array}$ & $\begin{array}{l}217.550 \\
218.550\end{array}$ & $\begin{array}{l}0.9239 \\
0.8192\end{array}-192$ & $\begin{array}{l}44.04235 \\
43: 64023\end{array}$ & $\begin{array}{l}175,37 \\
44,24\end{array}$ & $\begin{array}{l}1163.84 \\
292.84\end{array}$ & $\begin{array}{l}17516 \\
17616\end{array}$ & $\begin{array}{l}15810 \\
14097\end{array}$ & $\begin{array}{l}0.1762 \\
0.1770\end{array}$ & $\begin{array}{l}485 \\
470\end{array}$ & $\begin{array}{l}485 \\
470\end{array}$ & \\
\hline 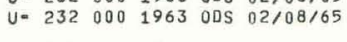 & 219,550 & 0.7777 & 43.24360 & 557.46 & 3665,83 & 17437 & 13247 & 0.1778 & 376 & 376 & \\
\hline $\begin{array}{lll}5 & 022 \\
5 & 022\end{array}$ & $\begin{array}{l}220.550 \\
221.550\end{array}$ & 0.95 & $\begin{array}{l}42.85534 \\
42.46637\end{array}$ & $\begin{array}{l}292,65 \\
104.70\end{array}$ & $\begin{array}{r}1915.72 \\
682.30\end{array}$ & $\begin{array}{l}17707 \\
17611\end{array}$ & & & $\begin{array}{l}372 \\
479\end{array}$ & $\begin{array}{l}372 \\
479\end{array}$ & \\
\hline 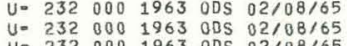 & $\begin{array}{l}222.550 \\
223.550\end{array}$ & 0.9910 & $\begin{array}{l}42,08850 \\
41.70992\end{array}$ & $\begin{array}{l}51.68 \\
20.09\end{array}-19$ & $\begin{array}{l}335,26 \\
129.73\end{array}$ & $\begin{array}{l}17737 \\
17721\end{array}$ & $\begin{array}{l}16926 \\
17159\end{array}-125$ & $\begin{array}{l}0,1803 \\
0,1811\end{array}$ & $\begin{array}{l}350 \\
383\end{array}$ & $\begin{array}{l}350 \\
383\end{array}$ & \\
\hline 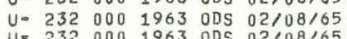 & $\begin{array}{l}224.550 \\
225.550 \\
550\end{array}$ & $\begin{array}{l}0.9858 \\
0.9843\end{array}$ & $\begin{array}{l}41.3525 \\
40.97350\end{array}$ & 35.16 & $\begin{aligned} 225.06 \\
.25 .06\end{aligned}$ & $\begin{array}{l}17509 \\
17574\end{array}$ & $\begin{array}{l}10861 \\
16898\end{array}$ & $\begin{array}{l}0.1819 \\
0.1827\end{array}$ & 373 & 373 & \\
\hline $\begin{array}{l}2 \\
2000 \\
2 \\
0\end{array}$ & $\begin{array}{l}226,550 \\
227.520 \\
550\end{array}$ & $\begin{array}{l}1,0077 \\
0,9921\end{array}$ & $\begin{array}{l}440.6158 \\
40.2561\end{array}$ & $\begin{array}{l}-177.00 \\
17.67\end{array}$ & $\begin{array}{l}-108,32 \\
112,11\end{array}$ & $\begin{array}{l}11447 \\
17530\end{array}$ & & & $\begin{array}{l}413 \\
406\end{array}$ & $\begin{array}{l}413 \\
406 \\
406\end{array}$ & \\
\hline 2320001963 ons $02 / 08 / 65$ & $\begin{array}{l}228,550 \\
229,550\end{array}$ & $\begin{array}{l}0.9943 \\
0.9796\end{array}$ & $\begin{array}{l}39.90940 \\
39.5579 B\end{array}$ & $\begin{array}{l}12,58 \\
45,74\end{array}-20$ & $\begin{array}{l}79 ; 45 \\
287,67\end{array}$ & $\begin{array}{l}17376 \\
17284\end{array}$ & $\begin{array}{l}168 \pi \varepsilon \\
16540\end{array}$ & $\begin{array}{l}0,1851 \\
0.1859\end{array}$ & $\begin{array}{l}590 \\
293\end{array}$ & $\begin{array}{l}\begin{array}{l}340 \\
293\end{array} \\
2930\end{array}$ & \\
\hline $\begin{array}{ll}01963 \text { ODS } 02 / 08 / 65 \\
0\end{array}$ & $\begin{array}{r}230.5 \\
231\end{array}$ & 1,0008 & 39.21556 & -1.85 & & 16913 & & & 351 & 351 & \\
\hline $\begin{array}{l}212808755 \\
2708 / 65\end{array}$ & $\begin{array}{l}231,550 \\
232,550\end{array}$ & 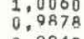 & $\begin{array}{l}38,8757 \\
38 ; 5493\end{array}$ & $\begin{array}{l}-133.22 \\
27,18\end{array}$ & $\begin{array}{l}-822.43 \\
160.75\end{array}$ & $\begin{array}{l}17109 \\
17361\end{array}$ & $\begin{array}{l}1669 \\
162\end{array}$ & $\begin{array}{l}0,1876 \\
0,1884\end{array}$ & $\begin{array}{l}\begin{array}{l}325 \\
341\end{array} \\
3\end{array}-10$ & $\begin{array}{l}325 \\
341\end{array}$ & \\
\hline $\begin{array}{l}02 / 18 / 65 \\
02 / 08 / 65\end{array}$ & $\begin{array}{l}2333.550 \\
234.550\end{array}$ & $\begin{array}{l}0.9942 \\
0,9762\end{array}$ & $\begin{array}{l}388.2157 \\
37.88941\end{array}$ & $\begin{array}{l}12.93 \\
53.48\end{array}$ & $\begin{array}{l}79.93 \\
329: 19\end{array}$ & $\begin{array}{l}17386 \\
17490\end{array}$ & $\begin{array}{l}16805 \\
16675\end{array}-3$ & $\begin{array}{l}0.1892 \\
0.1900\end{array}$ & $\begin{array}{l}403 \\
365\end{array}$ & $\begin{array}{l}403 \\
365\end{array}$ & \\
\hline 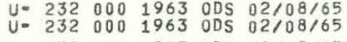 & $\begin{array}{l}2355.550 \\
236.550\end{array}$ & $\begin{array}{l}0.9968 \\
0,9839\end{array}-19$ & $\begin{array}{l}37.563838 \\
37: 25142\end{array}$ & $\begin{array}{l}7: 07 \\
36: 03\end{array}$ & $\begin{array}{l}413.34 \\
219: 92\end{array}$ & $\begin{array}{l}17388 \\
17588\end{array}$ & $\begin{array}{l}16932 \\
16904\end{array}$ & $\begin{array}{l}0.1908 \\
0: 1916\end{array}$ & $\begin{array}{l}375 \\
250\end{array}$ & $\begin{array}{l}\begin{array}{l}375 \\
250\end{array} \\
250-10\end{array}$ & \\
\hline 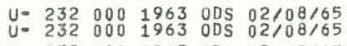 & $\begin{array}{l}237.550 \\
238.550\end{array}$ & $\begin{array}{l}0,9877 \\
0: 9758\end{array}$ & $\begin{array}{l}36: 93845 \\
36: 62940\end{array}$ & $\begin{array}{l}27: 50 \\
54: 20\end{array}$ & $\begin{array}{l}167: 13 \\
328: 04\end{array}$ & $\begin{array}{l}17332 \\
17211\end{array}$ & $\begin{array}{l}16722 \\
16407\end{array}$ & $\begin{array}{lll}0 & 1994 \\
0: 1932 & \end{array}$ & $\begin{array}{l}3449 \\
397\end{array}$ & 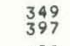 & \\
\hline 20001963 ODS $02 / 08 / 65$ & 239.520 & 1,0022 & 36.32422 & $-4,80$ & & 16825 & $1647 \mathrm{a}$ & 940 & 400 & 400 & \\
\hline 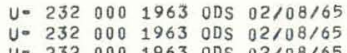 & $\begin{array}{l}240 \\
241\end{array}$ & $\begin{array}{l}0,9896 \\
0.9871\end{array}$ & $\begin{array}{l}36.02284 \\
35.72519\end{array}$ & $\begin{array}{l}23.14 \\
28.85\end{array}$ & 38 & $\begin{array}{l}16999 \\
11049\end{array}$ & $\begin{array}{l}16434 \\
16439\end{array}$ & & $\begin{array}{l}352 \\
345 \\
3450\end{array}-2$ & $\begin{array}{l}352 \\
345\end{array}$ & \\
\hline $\begin{array}{llll}232 & 000 & 1963 & 00 \\
32 & 000 & 1963 & 0 D\end{array}$ & $\begin{array}{l}242,520 \\
243,5>0\end{array}$ & & $\begin{array}{l}35.43122 \\
35: 14086\end{array}$ & $\begin{array}{l}25.75 \\
22.35\end{array}-\mathrm{l}$ & & $\begin{array}{l}16923 \\
16958\end{array}$ & $\begin{array}{l}16341 \\
16395\end{array}-\mathrm{l}$ & & $\begin{array}{l}301 \\
345\end{array}$ & $\begin{array}{l}301 \\
345\end{array}$ & \\
\hline 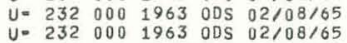 & $\begin{array}{l}244,550 \\
245,550\end{array}$ & 9887 & $\begin{array}{l}34.85406 \\
34,57757\end{array}$ & $\begin{array}{r}25.26 \\
117.12\end{array}$ & $\begin{array}{l}149.14 \\
774.42\end{array}$ & $\begin{array}{l}16988 \\
11043\end{array}$ & $\begin{array}{l}16407 \\
15721\end{array}$ & & $\begin{array}{l}318 \\
279\end{array}$ & $\begin{array}{l}318 \\
279\end{array}$ & \\
\hline 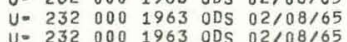 & $\begin{array}{l}246.550 \\
247550\end{array}$ & 0.9360 & $\begin{array}{l}34.29088 \\
34.01408\end{array}$ & $\begin{array}{l}146.61 \\
146.61\end{array}$ & $\begin{array}{l}858.54 \\
255.54\end{array}$ & $\begin{array}{l}17138 \\
17738\end{array}$ & $\begin{array}{l}15670 \\
15647\end{array}-20$ & 0.0 .1997 & $\begin{array}{l}295 \\
295 \\
305\end{array}$ & $\begin{array}{l}295 \\
205 \\
335\end{array}$ & \\
\hline 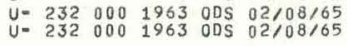 & $\begin{array}{l}248,550 \\
249,550\end{array}$ & $\begin{array}{l}0,9946 \\
0,9776\end{array}$ & $\begin{array}{l}333,74125 \\
33 ; 47137\end{array}$ & $\begin{array}{l}11: 94 \\
50: 19\end{array}$ & $\begin{array}{l}69.37 \\
290.34\end{array}$ & $\begin{array}{l}16905 \\
117346\end{array}$ & $\begin{array}{l}16425 \\
16566\end{array}$ & $\begin{array}{l}0,2013 \\
0 ; 2021\end{array}$ & $\begin{array}{l}354 \\
314\end{array}$ & $\begin{array}{l}354 \\
314\end{array}$ & \\
\hline 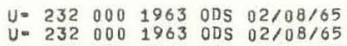 & $\begin{array}{l}250,550 \\
252.050\end{array}$ & $\begin{array}{l}0.9972 \\
0.9829\end{array}-19$ & $\begin{array}{l}33,20472 \\
32,81068\end{array}$ & $\begin{array}{l}6.13 \\
38.24\end{array}$ & $\begin{array}{l}5.35 \\
9.03 \\
0.03\end{array}$ & $\frac{16930}{3005}$ & $\begin{array}{l}16493 \\
32727\end{array}$ & $\begin{array}{l}0,2029 \\
0 ; 2042\end{array}$ & $\begin{array}{l}327 \\
682\end{array}$ & $\begin{array}{l}327 \\
662\end{array}$ & \\
\hline $\begin{array}{l}1963 \text { ODS } 02 / 08 / 65 \\
1963 \text { ODS } 02 / 101865\end{array}$ & $\begin{array}{l}254,050 \\
256,050\end{array}$ & $\begin{array}{l}9950 \\
9823\end{array}$ & $\begin{array}{l}32,29612 \\
331,79356\end{array}$ & $\begin{array}{l}11,20 \\
39,52\end{array}$ & $\begin{array}{l}3.63 \\
2.86\end{array}$ & $\begin{array}{l}33962 \\
33470\end{array}$ & $\begin{array}{l}33005 \\
32790\end{array}$ & 0. & $\begin{array}{l}6359 \\
536\end{array}$ & $\begin{array}{l}6359 \\
536\end{array}$ & \\
\hline 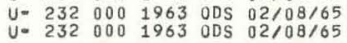 & $\begin{array}{l}258,050 \\
260: 300\end{array}$ & $\begin{array}{l}0.9866 \\
0,9973\end{array}-9973$ & $\begin{array}{l}31.30264 \\
30: 76383\end{array}$ & $\begin{array}{r}29.85 \\
6.00\end{array}$ & $\begin{array}{c}167.03 \\
33.26\end{array}$ & $\begin{array}{l}33953 \\
44638\end{array}$ & $\begin{array}{l}32724 \\
4348 \mathrm{E}\end{array}$ & $\begin{array}{l}0: 2090 \\
0 ; 2108\end{array}$ & $\begin{array}{l}609 \\
826\end{array}$ & $\begin{array}{l}609 \\
826\end{array}$ & \\
\hline 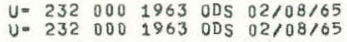 & $\begin{array}{l}262.550 \\
264: 550\end{array}$ & $\begin{array}{l}0,9733 \\
0: 9921\end{array}-1$ & $\begin{array}{l}30.23881 \\
29.78332\end{array}$ & $\begin{array}{l}59.90 \\
17.50\end{array}$ & $\begin{aligned} 329.38 \\
95.53\end{aligned}$ & $\begin{array}{l}34015 \\
33812\end{array}$ & $\begin{array}{l}32342 \\
32770\end{array}-100$ & $\begin{array}{l}0,2127 \\
0,2143\end{array}$ & $\begin{array}{l}593 \\
580\end{array}$ & $\begin{array}{l}593 \\
580\end{array}$ & \\
\hline 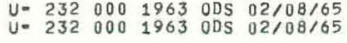 & $\begin{array}{l}266,800 \\
269,300\end{array}$ & $\begin{array}{l}0,9866 \\
0,9780\end{array}$ & $\begin{array}{l}29.28310 \\
28: 7493\end{array}$ & $\begin{array}{l}29,86 \\
49,22\end{array}-12$ & $\frac{161.59}{263.88}$ & $\begin{array}{l}39983 \\
44025\end{array}$ & $\begin{array}{l}37766 \mathrm{E} \\
428 a 6\end{array}$ & $\begin{array}{l}0.2161 \\
0 ; 2181\end{array}$ & $\begin{array}{l}645 \\
731\end{array}$ & $\begin{array}{l}645 \\
731\end{array}$ & \\
\hline no0 1963 ops $02 / 08 / 65$ & 271,050 & 771 & 28. & 1.3 & 10 & 50 & 15797 & 6 & 51 & 251 & \\
\hline 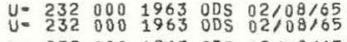 & $\begin{array}{l}2722: 050 \\
273: 050\end{array}$ & $\begin{array}{l}0.9382 \\
0,8312\end{array}$ & $\begin{array}{l}28 \\
27: 165789\end{array}$ & $\begin{array}{l}141.45 \\
410: 02\end{array}$ & $\begin{array}{l}7500: 70 \\
2167: 97\end{array}$ & $\begin{array}{l}15582 \\
14778\end{array}$ & 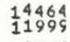 & $\begin{array}{l}0 \\
0: 22124 \\
0: 2212\end{array}$ & $\begin{array}{l}281 \\
330\end{array}$ & $\begin{array}{l}281 \\
330\end{array}$ & \\
\hline 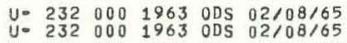 & $\begin{array}{l}274: 050 \\
275: 050\end{array}$ & $\begin{array}{l}0,7866 \\
0,8637\end{array}-17$ & $\begin{array}{l}27.75422 \\
27: 55278\end{array}$ & $\begin{array}{l}532: 16 \\
324: 77\end{array}$ & $\begin{array}{l}2803: 55 \\
1704: 72\end{array}$ & $\begin{array}{l}14193 \\
15345\end{array}$ & $\begin{array}{l}10906 \\
12947\end{array}$ & $\begin{array}{l}0 ; 2220 \\
0 ; 228\end{array}$ & $\begin{array}{l}265 \\
250\end{array}$ & $\begin{array}{l}265 \\
250\end{array}$ & \\
\hline 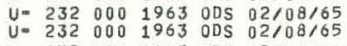 & $\begin{array}{l}276.050 \\
277 \% 090\end{array}$ & $\begin{array}{l}0: 9567 \\
0,9644\end{array}$ & $\begin{array}{l}27735352 \\
27 ; 15641\end{array}$ & $\begin{array}{l}98,08 \\
80,33\end{array}-1$ & $\begin{array}{l}512.95 \\
418: 59\end{array}$ & $\begin{array}{l}15586 \\
16404\end{array}$ & $\begin{array}{l}14940 \\
15454\end{array}$ & $\begin{array}{l}0.2236 \\
0.2244\end{array}$ & $\begin{array}{l}264 \\
242\end{array}$ & $\begin{array}{l}264 \\
242\end{array}$ & \\
\hline 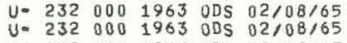 & $\begin{array}{l}278,380 \\
280,050\end{array}$ & $\begin{array}{l}0.9955 \\
0,9887\end{array}-190$ & $\begin{array}{l}26.91301 \\
26,57771\end{array}$ & $\begin{array}{l}10,05 \\
25,13\end{array}$ & $\begin{array}{r}52.14 \\
129 \\
1253\end{array}$ & $\begin{array}{l}21939 \\
33260\end{array}$ & $\begin{array}{l}21334 \\
32124\end{array}$ & ${ }_{8}^{4}$ & $\begin{array}{l}235 \\
545\end{array}$ & $\begin{array}{l}2355 \\
545\end{array}$ & \\
\hline 1320001963 ODS 02 & & 0.9867 & 26.20212 & 29. & 18 & & & & & & \\
\hline 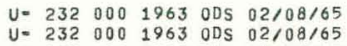 & $\begin{array}{l}284,050 \\
286,300\end{array}$ & $\begin{array}{l}0.99 \\
0.98\end{array}$ & $\begin{array}{l}25.83444 \\
25 ; 42998\end{array}$ & $\begin{array}{l}21.71 \\
30,83\end{array}$ & $\begin{array}{l}32 \\
47\end{array}$ & $\begin{array}{r}33078 \\
44211\end{array}$ & 31998 & & 491 & 491 & \\
\hline 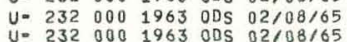 & $\begin{array}{l}288.800 \\
290.550\end{array}$ & 0.9743 & $\begin{array}{l}24.99162 \\
2406947\end{array}$ & $\begin{array}{l}70.69 \\
57.62\end{array}$ & $\begin{array}{l}355.41 \\
28.43 \\
28.31\end{array}$ & $\begin{array}{l}38634 \\
164037\end{array}$ & $\begin{array}{l}36556 \\
15625\end{array}-100$ & $\begin{array}{l}0.2339 \\
0.2353\end{array}$ & 535 & $\begin{array}{r}535 \\
576\end{array}$ & \\
\hline $502 / 108165$ & 291550 & & 24.52238 & 100.86 & & $\begin{array}{l}10617 \\
16508\end{array}$ & $\begin{array}{l}15409 \\
1540\end{array}$ & & 144 & 141 & \\
\hline 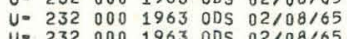 & 293.550 & 1 & 24.18937 & 402.25 & 1978.36 & & $\begin{array}{l}13312 \\
13316\end{array}$ & & 19 & $\begin{array}{l}245 \\
196\end{array}$ & \\
\hline & & & 29.02540 & 2047,07 & & & & & & 256 & \\
\hline & & & 23. & 1083 & & & & & 212 & & \\
\hline 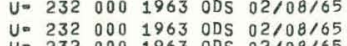 & $\begin{array}{l}297.550 \\
298.550\end{array}$ & $0_{0}^{0}-(-2-1$ & $\begin{array}{l}23.54 \\
23,38\end{array}$ & & & & & & 172 & $\begin{array}{l}172 \\
305\end{array}$ & \\
\hline 320001963 & & & & & & & & & & $\begin{array}{l}253 \\
203\end{array}$ & \\
\hline $02 / 08 / 65$ & & & $\begin{array}{ll}22.0292 \\
22.69656\end{array}$ & 60.04 & $\begin{array}{l}286.06 \\
280,00\end{array}$ & $\begin{array}{l}22485 \\
3246\end{array}$ & 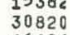 & & $\begin{array}{l}21 \\
48 \\
48\end{array}$ & $\begin{array}{l}213 \\
482\end{array}$ & \\
\hline $02 / 08 / 65$ & & & $\begin{array}{l}22.02088 \\
21.89463\end{array}$ & & & & & & & & \\
\hline & & & 21. & 119 & & & & & & & \\
\hline & & & & & & & & & & & \\
\hline 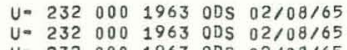 & $\begin{array}{l}312.54 \\
313.5\end{array}$ & 0,9 & $\begin{array}{l}21,33 \\
21,20\end{array} \mathrm{~V} \mathrm{~V}$ & $\begin{array}{l}1999: \\
342:\end{array}$ & & $\begin{array}{l}15717 \\
15733\end{array}$ & $\begin{array}{l}14 \\
13\end{array}$ & 2 & $\begin{array}{l}192 \\
160\end{array}$ & & \\
\hline 02108 & & 87 & $\begin{array}{l}21.06 \\
20.93\end{array}$ & $\begin{array}{l}347: 0 \\
29: 7\end{array}$ & $\begin{array}{ll}593 \\
371\end{array}$ & $\begin{array}{l}15720 \\
15902\end{array}$ & ${ }_{13}^{13}$ & & & $\begin{array}{l}185 \\
178\end{array}$ & \\
\hline 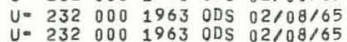 & $\begin{array}{l}316.5 \\
317.5\end{array}$ & 0.89 & $\begin{array}{l}20080195 \\
2006714\end{array}$ & $\begin{array}{l}237.5 \\
12,7\end{array}$ & & 16 & & & & 11 & \\
\hline 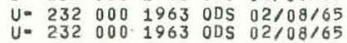 & $\begin{array}{l}318.5 \\
319 ; 5\end{array}$ & & $\begin{array}{l}20.54 \\
20: 41\end{array}$ & $\begin{array}{l}148.40 \\
142.10\end{array}$ & & 15 & & & 1 & $\begin{array}{l}169 \\
209\end{array}$ & \\
\hline 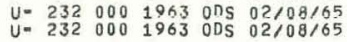 & $\begin{array}{l}3220.550 \\
321550\end{array}$ & $00,9741 \frac{1}{0 ; 9673}$ & $\begin{array}{l}20,28603 \\
20 ; 1005\end{array}$ & 12 & & 60 & & & $\begin{array}{l}\frac{183}{214} \\
214\end{array}-\mathrm{l}$ & 18 & \\
\hline
\end{tabular}




\begin{tabular}{|c|c|c|c|c|c|c|c|c|c|c|c|}
\hline IDENTIFICATION & DELAY & TRANS, & ENERGY & $x$-SEC & $\begin{array}{l}x=S E C \\
R O O T E\end{array}$ & OPEN & $\begin{array}{l}\text { IS } \\
\text { SAMP }\end{array}$ & $\begin{array}{l}\text { FRAC, } \\
\text { OF OCT }\end{array}$ & $\begin{array}{l}\text { FAST } \\
\text { OPEN }\end{array}$ & GS & $\begin{array}{l}\text { Oa PTS } \\
\text { AVE, }\end{array}$ \\
\hline $\begin{array}{l}\text { ODS } 02 / 08 / 65 \\
\text { ODS } 02 / 08 / 65\end{array}$ & $\begin{array}{l}322.550 \\
324.050\end{array}$ & $\begin{array}{l}0,9786 \\
0,9848\end{array}$ & $\begin{array}{l}20.03524 \\
19: 85019\end{array}$ & $\begin{array}{l}48.02 \\
33.89\end{array}$ & $\begin{array}{l}214.95 \\
150.98\end{array}$ & $\begin{array}{l}15711 \\
31163\end{array}$ & $\begin{array}{l}15019 \\
29980\end{array}$ & $\begin{array}{l}0.2613 \\
0.2625\end{array}$ & $\begin{array}{l}164 \\
400\end{array}$ & $\begin{array}{l}164 \\
408\end{array}$ & \\
\hline $\begin{array}{l}\text { DSS } 02208065 \\
\text { DS } 02 / 08 / 65\end{array}$ & $\begin{array}{l}326: 300 \\
322 ; 800\end{array}$ & $\begin{array}{l}0,981 \\
0 ; 9803 \\
0 ; 803\end{array}$ & $\begin{array}{l}199.57738 \\
19.28080\end{array}$ & $\begin{array}{l}44 ; 48 \\
44: 14\end{array}$ & $\begin{array}{l}196,87 \\
193 ; 82\end{array}$ & $\begin{array}{l}41500 \\
35445\end{array}$ & $\begin{array}{l}39934 \\
33948\end{array}$ & $\begin{array}{l}0.2643 \\
0.2663\end{array}-3$ & 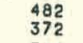 & $\begin{array}{l}482 \\
372\end{array}$ & \\
\hline 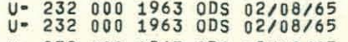 & $\begin{array}{l}331 \\
334.5500 \\
300\end{array}$ & $\begin{array}{l}0 \\
0: 97784 \\
0: 9787\end{array}$ & $\begin{array}{l}18 \\
18: 65159 \\
85159\end{array}$ & $\begin{array}{l}59,76 \\
47,76\end{array}$ & $\begin{array}{l}200,25 \\
206.25\end{array}$ & $\begin{array}{l}45219 \\
40749\end{array}$ & $\begin{array}{l}42997 \\
38957\end{array}$ & $\begin{array}{l}0.2686 \\
0,2708\end{array}$ & $\begin{array}{l}536 \\
411\end{array}$ & $\begin{array}{l}536 \\
411\end{array}$ & \\
\hline 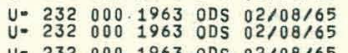 & $\begin{array}{l}336,800 \\
339,550\end{array}$ & $\begin{array}{l}0: 9910 \\
0.9923\end{array}$ & $\begin{array}{l}18,37573 \\
118.0728\end{array}$ & $\begin{array}{l}19,98 \\
17.15\end{array}$ & $\begin{array}{l}85.63 \\
72: 91\end{array}$ & $\begin{array}{l}34988 \\
46064\end{array}$ & $\begin{array}{l}33871 \\
44651\end{array}$ & $\begin{array}{l}0: 2728 \\
0: 2750\end{array}$ & $\begin{array}{l}384 \\
431\end{array}$ & $\begin{array}{l}384 \\
431\end{array}$ & \\
\hline 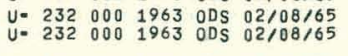 & $\begin{array}{l}342.550 \\
345.550\end{array}$ & $\begin{array}{l}0: 9820 \\
0,9904\end{array}$ & $\begin{array}{l}17,76400 \\
17,45689\end{array}$ & $\begin{array}{l}40.31 \\
21,35\end{array}$ & $\begin{array}{r}169.90 \\
89.20\end{array}$ & $\begin{array}{l}\begin{array}{l}46230 \\
45486\end{array} \\
4540\end{array}$ & $\begin{array}{l}44346 \\
44007\end{array}$ & $\begin{array}{l}0.2775 \\
0.2799\end{array}$ & $\begin{array}{l}457 \\
433\end{array}$ & $\begin{array}{l}457 \\
433\end{array}$ & \\
\hline U. 2320001963 ODS $02 / 08 / 65$ & $\begin{array}{l}348.550 \\
35.550\end{array}$ & 0,9866 & 17.15768 & 29,95 & 124.06 & 45755 & 44096 & & 363 & 363 & \\
\hline 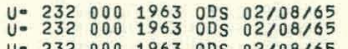 & $\begin{array}{l}351,550 \\
354: 840 \\
358\end{array}$ & $\begin{array}{l}0: 9949 \\
0: 9981 \\
0.975\end{array}$ & $\begin{array}{l}16.86609 \\
16: 55852\end{array}$ & $\begin{array}{l}11.25 \\
4.31 \\
57.28\end{array}$ & $\begin{array}{r}46: 19 \\
17: 54\end{array}$ & $\begin{array}{l}45321 \\
55098\end{array}$ & $\begin{array}{l}44047 \\
53718 \\
43148\end{array}$ & $\begin{array}{l}0,288 \\
0: 2874 \\
0.2907\end{array}$ & $\begin{array}{l}374 \\
528 \\
382\end{array}$ & $\begin{array}{l}374 \\
528 \\
382\end{array}$ & \\
\hline 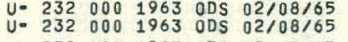 & $\begin{array}{l}358.050 \\
361: 300\end{array}$ & $\begin{array}{l}0: 975 \\
0: 9863\end{array}$ & $\begin{array}{l}166.25258 \\
15.96808\end{array}$ & $\begin{array}{l}57: 28 \\
30: 59\end{array}$ & $\begin{array}{l}230.97 \\
122.24\end{array}$ & 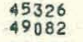 & $\begin{array}{l}413148 \\
47285\end{array}$ & $\begin{array}{l}0.2900 \\
0 ; 2927\end{array}$ & $\begin{array}{l}382 \\
350\end{array}$ & $\begin{array}{l}382 \\
350\end{array}$ & \\
\hline 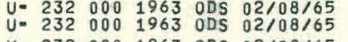 & $\begin{array}{l}364: 800 \\
368: 300\end{array}$ & $\begin{array}{l}0: 9759 \\
0: 9874\end{array}$ & $\begin{array}{l}155: 66315 \\
15: 36686\end{array}$ & $\begin{array}{l}54.88 \\
28.20\end{array}$ & $\begin{array}{l}214.044 \\
110: 54\end{array}$ & $\begin{array}{l}54939 \\
48813\end{array}$ & $\begin{array}{l}53237 \\
47080\end{array}$ & $\begin{array}{l}0.255 \\
0 ; 2983\end{array}$ & $\begin{array}{l}470 \\
319\end{array}$ & $\begin{array}{l}470 \\
319\end{array}$ & \\
\hline 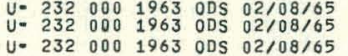 & $\begin{array}{l}372,050 \\
375 ; 800 \\
370\end{array}$ & $\begin{array}{l}0.983 \\
0 ; 9688\end{array}$ & $\begin{array}{l}15.05585 \\
14: 75962\end{array}$ & $\begin{array}{l}44.22 \\
20.21 \\
2.21\end{array}$ & $\begin{array}{l}171.59 \\
269.73\end{array}$ & $\begin{array}{l}55063 \\
54159\end{array}$ & $\begin{array}{l}55556 \\
51255\end{array}$ & $\begin{array}{l}0: 314 \\
0: 3044 \\
0\end{array}$ & 398 & $\begin{array}{l}366 \\
398 \\
398\end{array}$ & \\
\hline $\begin{array}{l}1963 \text { ODS } 02 / 08 / 65 \\
1963 \text { ODS } 02 / 08 / 65\end{array}$ & 379,550 & 0.9796 & 14.46941 & 45,66 & & 57600 & 55120 & & 375 & 375 & \\
\hline 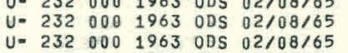 & $\begin{array}{l}386.050 \\
387.050\end{array}$ & $\begin{array}{l}0,986 \\
0,940 \\
0,9711\end{array}$ & $\begin{array}{l}114.16918 \\
13.98626 \\
13.91408\end{array}$ & $\begin{array}{r}10.80 \\
123.10 \\
64.99\end{array}$ & : & $\begin{array}{l}57493 \\
14334 \\
14181\end{array}$ & $\begin{array}{l}54396 \\
13246 \\
13456\end{array}$ & 7 & $\begin{array}{l}406 \\
101 \\
109\end{array}$ & $\begin{array}{l}406 \\
101\end{array}$ & \\
\hline 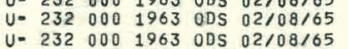 & $\begin{array}{l}388,020 \\
389,300\end{array}$ & $\begin{array}{l}0.911 \\
0: 924 \\
0.9292\end{array}$ & 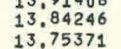 & $\begin{array}{l}134.62 \\
131.62\end{array}$ & $\begin{array}{l}249 \\
489.71 \\
6047\end{array}$ & $\begin{array}{l}11431 \\
14381\end{array}$ & 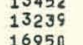 & 集 & $\begin{array}{r}96 \\
54 \\
.143\end{array}$ & $\begin{array}{r}98 \\
54 \\
.43\end{array}$ & \\
\hline 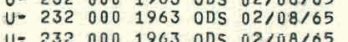 & $\begin{array}{l}390.550 \\
301\end{array}$ & $\begin{array}{ll}0.9534 \\
0.954\end{array}$ & $\begin{array}{l}133.66581 \\
133\end{array}$ & $\begin{array}{l}105.90 \\
105.91\end{array}$ & $\begin{array}{ll}391.53 \\
713\end{array}$ & $\begin{array}{l}11212 \\
14212\end{array}$ & 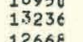 & $0:$ & 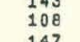 & $\begin{array}{l}145 \\
108\end{array}$ & \\
\hline 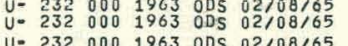 & $\begin{array}{l}391.550 \\
392,550 \\
303\end{array}$ & $\begin{array}{l}0: 9164 \\
0: 9016 \\
0.9093\end{array}$ & 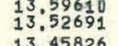 & $\begin{array}{l}1139,63 \\
229,65 \\
2352\end{array}$ & 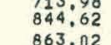 & $\begin{array}{l}14337 \\
14277\end{array}$ & 年 & 80 & 68 & $\begin{array}{l}1.8 \\
68 \\
108\end{array}$ & \\
\hline 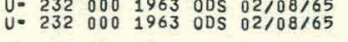 & $\begin{array}{l}3933.520 \\
394.550\end{array}$ & $\begin{array}{l}0: 893 \\
0 ; 812 \\
0.812\end{array}$ & $\begin{array}{l}133.45826 \\
13: 39012\end{array}$ & $\begin{array}{l}285.55 \\
280.28\end{array}$ & $\begin{array}{l}0025,60 \\
1025\end{array}$ & $\begin{array}{l}14160 \\
14160\end{array}-100$ & 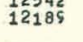 & 3196 & 139 & 139 & \\
\hline $\begin{array}{l}2320001963 \text { ODS } 02 / 08 / 65 \\
\end{array}$ & $\begin{array}{r}395.550 \\
396\end{array}$ & 0.8457 & 13,32251 & 371,46 & 5,81 & 14341 & 11848 & 3204 & 99 & 99 & \\
\hline 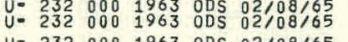 & $\begin{array}{l}3996: 550 \\
397,550\end{array}$ & $\begin{array}{l}0.8149 \\
0: 8049\end{array}$ & $\begin{array}{l}133 \\
13: 18580 \\
13\end{array}$ & $\begin{array}{l}453: 89 \\
481 ; 25\end{array}$ & $\begin{array}{l}1652: 53 \\
1747: 72\end{array}$ & 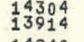 & $\begin{array}{l}11385 \\
10935\end{array}$ & 20 & 88 & $\begin{array}{l}89 \\
83\end{array}$ & \\
\hline 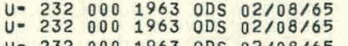 & $\begin{array}{l}398,550 \\
399: 550\end{array}$ & $\begin{array}{l}007226 \\
0: 6736\end{array}$ & $\begin{array}{l}133.12270 \\
13.05709\end{array}$ & $\begin{array}{l}770: 41 \\
876: 09\end{array}$ & $\begin{array}{l}2609: 69 \\
3165 ; 72\end{array}$ & $\begin{array}{l}1240 \\
14018\end{array}$ & $\begin{array}{l}10051 \\
9223 \\
923\end{array}$ & $\begin{array}{l}0,328 \\
0: 3236\end{array}$ & $\begin{array}{l}67 \\
94\end{array}$ & 94 & \\
\hline 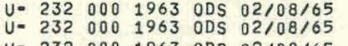 & $\begin{array}{l}400.550 \\
401,520\end{array}$ & $\begin{array}{l}0,5602 \\
0,4455\end{array}$ & $\begin{array}{l}122,99198 \\
12,92735\end{array}$ & $\begin{array}{l}128,85 \\
1792: 79\end{array}$ & $\begin{array}{l}\begin{array}{l}4631: 15 \\
6445: 89\end{array} \\
69\end{array}$ & $\begin{array}{l}14122 \\
13965\end{array}$ & $\begin{array}{l}7727 \\
6085\end{array}$ & $\begin{array}{l}0,3244 \\
0 ; 3253\end{array}$ & $\begin{array}{r}83 \\
109\end{array}$ & $\begin{array}{r}83 \\
109\end{array}$ & \\
\hline 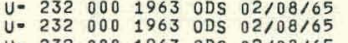 & $\begin{array}{l}402,550 \\
403 \% 550\end{array}$ & $\begin{array}{l}0.3371 \\
0.2452\end{array}$ & $\begin{array}{l}12,863200 \\
12,79953\end{array}$ & $\begin{array}{l}24410,85 \\
3116,32\end{array}$ & $\begin{aligned} 8646,59 \\
11149: 07\end{aligned}$ & $\begin{array}{l}\frac{138983}{13892} \\
1382-10\end{array}$ & $\begin{array}{l}4574 \\
3327\end{array}$ & $\begin{array}{l}0,3261 \\
0 ; 3269\end{array}$ & $\begin{array}{l}87 \\
67\end{array}$ & $\begin{array}{l}87 \\
67\end{array}$ & \\
\hline $02 / 08 / 65$ & $\begin{array}{l}404,5 \\
405,5\end{array}$ & & $\begin{array}{l}12,7 \\
12,6\end{array}$ & & & 13984 & 2777 & & 9 & 19 & \\
\hline $\begin{array}{l}\text { S } 021 / 108 / 65 \\
\text { s } 02 / 08 / 65\end{array}$ & $\begin{array}{l}406,550 \\
407,550\end{array}$ & $\begin{array}{ll}0.3138 \\
0 ; 4324\end{array}$ & $\begin{array}{l}12.61133 \\
12.5451\end{array}$ & $\begin{array}{l}256,25 \\
1568,25\end{array}$ & $\begin{array}{l}9124.04 \\
6053794\end{array}$ & $\begin{array}{l}13779 \\
13711\end{array}$ & $\begin{array}{l}5191 \\
4224 \\
5798\end{array}$ & 0 & 87 & 87 & \\
\hline 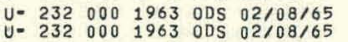 & $\begin{array}{l}408,550 \\
409 ; 550\end{array}$ & $\begin{array}{l}0.5617 \\
0.0686\end{array}$ & $\begin{array}{l}12,48815 \\
12 ; 42724\end{array}$ & $\begin{array}{l}1278,72 \\
892,39\end{array}$ & $\begin{array}{l}4516,82 \\
434588\end{array}$ & $\begin{array}{l}13848 \\
13927\end{array}$ & 7598 & $\begin{array}{l}0.3309 \\
0.3317\end{array}$ & $\begin{array}{l}42 \\
43 \\
43\end{array}$ & 42 & \\
\hline & $\begin{array}{l}140.330 \\
0.550\end{array}$ & & 12.36678 & 679. & & 3.0800 & 8 & & 01 & 61 & \\
\hline 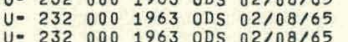 & $\begin{array}{l}41,550 \\
412,550\end{array}$ & 0.8265 & $\begin{array}{l}12,2416 \\
12,2816\end{array}$ & $\begin{array}{l}5 / 1.19 \\
422.49\end{array}$ & $\begin{array}{l}2003.99 \\
11478.55\end{array}$ & $\begin{array}{l}13997 \\
13973\end{array}$ & $\begin{array}{l}10567 \\
11281\end{array}$ & & 96 & 76 & \\
\hline 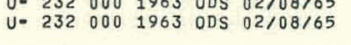 & $\begin{array}{l}413.550 \\
414.550\end{array}$ & 0.9025 & $\begin{array}{l}12,18801 \\
12,12928\end{array}$ & $\begin{array}{l}338.21 \\
227.54\end{array}$ & $\begin{array}{l}1180,75 \\
792,45\end{array}$ & $\begin{array}{l}13996 \\
13737\end{array}$ & $\begin{array}{l}738 \\
110\end{array}$ & 3358 & 02 & ${ }_{80}^{102}$ & \\
\hline 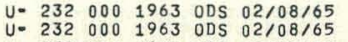 & $\begin{array}{l}415,550 \\
416,550\end{array}$ & $\begin{array}{l}0,8922 \\
0 ; 9055\end{array}$ & $\begin{array}{l}12,07097 \\
12,01308\end{array}$ & $\begin{array}{l}235,67 \\
22010\end{array}$ & $\begin{array}{l}818,80 \\
762.86\end{array}$ & $\begin{array}{l}13723 \\
13715\end{array}$ & $\begin{array}{l}12053 \\
12131\end{array}$ & $\begin{array}{l}3366 \\
374\end{array}$ & $\begin{array}{l}142 \\
101\end{array}$ & $\begin{array}{l}142 \\
101\end{array}$ & \\
\hline 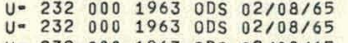 & $\begin{array}{l}417.550 \\
418.550\end{array}$ & $\begin{array}{l}0.9207 \\
0: 9497\end{array}$ & $\begin{array}{l}11.95561 \\
11.99855\end{array}$ & $\begin{array}{l}183,09 \\
114,42\end{array}$ & 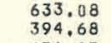 & $\begin{array}{l}13740 \\
13433\end{array}$ & $1246 \overline{8}$ & $0^{\circ}$ & 140 & 140 & \\
\hline 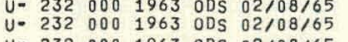 & $\begin{array}{l}419,550 \\
420.550\end{array}$ & $\begin{array}{l}0: 9450 \\
0: 9658\end{array}$ & $\begin{array}{l}\begin{array}{l}11.84900 \\
11: 78565\end{array} \\
\end{array}$ & $\begin{aligned} 125,52 \\
77,08\end{aligned}$ & $\begin{array}{l}431.95 \\
264.63\end{array}$ & $\begin{array}{l}13830 \\
13414\end{array}$ & $\begin{array}{l}12766 \\
12656\end{array}$ & $\begin{array}{l}98 \\
0.6\end{array}$ & $\begin{array}{l}76 \\
87\end{array}$ & $\begin{array}{l}76 \\
87\end{array}$ & \\
\hline 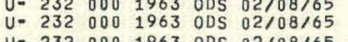 & $\begin{array}{l}421,550 \\
422,550\end{array}$ & $\begin{array}{l}0.9605 \\
0.9690\end{array}$ & $\begin{array}{l}11,79980 \\
11.6734 \\
7544\end{array}$ & $\begin{array}{l}89 ; 40 \\
69.81\end{array}$ & $\begin{array}{l}306.20 \\
238.51\end{array}$ & $\begin{array}{l}13512 \\
13683\end{array}$ & $\begin{array}{l}1267 \mathrm{E} \\
12952\end{array}$ & $\begin{array}{l}15 \\
23\end{array}$ & $\begin{array}{c}114 \\
61 \\
61\end{array}$ & $\underset{61}{114}$ & \\
\hline $\begin{array}{lllll}232 & 000 & 1963 & 005 & 02 \prime \\
232 & 000 & 1963 & 005 & 021\end{array}$ & $\begin{array}{l}424,860 \\
428,300\end{array}$ & $\begin{array}{l}0: 9673 \\
0.9732\end{array}$ & $\begin{array}{l}11,55100 \\
11 ; 36299\end{array}$ & $\begin{array}{l}73,77 \\
60,31\end{array}$ & $\begin{array}{l}250.74 \\
203.30\end{array}$ & $\begin{array}{l}50199 \\
44597\end{array}$ & 394 & $\begin{array}{l}441 \\
469\end{array}$ & $\begin{array}{l}332 \\
168\end{array}$ & $\begin{array}{l}332 \\
168\end{array}$ & \\
\hline 2000 1963 ons $02 / 08 / 65$ & & 0,9866 & 11.16 & 31. & .69 & 3213 & 1 & & ${ }^{317}$ & 317 & \\
\hline 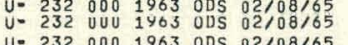 & $\begin{array}{l}436,050 \\
440,050 \\
40.300\end{array}$ & $\begin{array}{l}0: 936 \\
0: 964 \\
0860\end{array}$ & $\begin{array}{l}110: 96666 \\
10: 7627 \\
5527\end{array}$ & $\begin{array}{l}59: 2 \\
30,3 \\
3\end{array}$ & 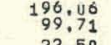 & $\begin{array}{l}53162 \\
52938\end{array}$ & $\begin{array}{l}50562 \\
5100 \\
5002\end{array}$ & & 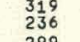 & $\begin{array}{l}3 \\
2 \\
2\end{array}$ & \\
\hline 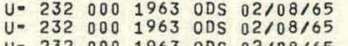 & $\begin{array}{l}44,300 \\
448.550\end{array}$ & 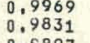 & $\begin{array}{l}10.5532 \\
10.36017\end{array}$ & 37,89 & $\begin{array}{r}22,50 \\
121.90\end{array}$ & $\begin{array}{l}60770 \\
50185\end{array}$ & $\begin{array}{l}59171 \\
48191 \\
4\end{array}$ & ; 36033 & 7 & $\begin{array}{l}299 \\
317\end{array}$ & \\
\hline 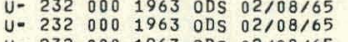 & $\begin{array}{l}452,800 \\
457,300\end{array}$ & $\begin{array}{l}0,597 \\
0,9972\end{array}$ & $\begin{aligned} 10.16660 \\
9.96750\end{aligned}$ & $\begin{array}{r}22,9 \\
6,1\end{array}$ & $\begin{array}{l}73.03 \\
19.32\end{array}$ & $\begin{array}{l}54929 \\
59858\end{array}-1010$ & $\begin{array}{l}53105 \\
58305\end{array}$ & $\begin{array}{l}0,0368 \\
0 ; 3704\end{array}$ & $\begin{array}{l}340 \\
294\end{array}$ & $\begin{array}{l}340 \\
294\end{array}$ & \\
\hline $\begin{array}{lll}232 & 000 & 1965 \\
232 & 000 & 196\end{array}$ & $\begin{array}{l}462,050 \\
467,0050\end{array}$ & & 9.55 & $\begin{array}{l}11,49 \\
18,43\end{array}$ & $\begin{array}{l}1.96 \\
96\end{array}$ & $\begin{array}{l}62972 \\
62340\end{array}$ & & & $\begin{array}{l}394 \\
449\end{array}$ & $\begin{array}{l}394 \\
449\end{array}$ & \\
\hline $232000 \quad 1963$ & 472,050 & 0,9872 & 9,35433 & 28,5 & & & & & 239 & 239 & \\
\hline 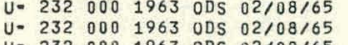 & $\begin{array}{l}4777.050 \\
482.300\end{array}-300$ & $\begin{array}{l}0.9922 \\
0.9861\end{array}$ & $\begin{array}{l}9.1 .152727 \\
8.96095\end{array}$ & $\begin{array}{l}17,2 \\
31,1\end{array}$ & 52.24 & $\begin{array}{l}61971 \\
64385\end{array}$ & & & 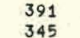 & $\begin{array}{l}91 \\
45\end{array}$ & \\
\hline 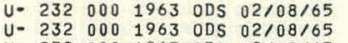 & $\begin{array}{l}4877800 \\
493,300\end{array}$ & $\begin{array}{l}0.9921 \\
0,9969\end{array}$ & $\begin{array}{l}8.76002 \\
8.56577\end{array}$ & 17.: & 20.08 & $\begin{array}{l}69230 \\
62807\end{array}$ & & & 351 & $\begin{array}{l}383 \\
351\end{array}$ & \\
\hline 021 & 502,530 & & & $-2 \quad 2>>$ & & & & & 31 & 314 & \\
\hline 232 & 503.550 & 0.9833 & 8.220 & $36:$ & & 11706 & & & & & \\
\hline 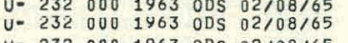 & $\begin{array}{l}50.550 \\
505.550\end{array}$ & 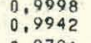 & 8.15805 & $\begin{array}{c}0,3 \\
12.8\end{array}$ & $\begin{array}{l}11.11 \\
36.68\end{array}$ & 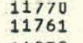 & $\begin{array}{l}11492 \\
11422\end{array}$ & (4) & $\begin{array}{l}88 \\
40\end{array}$ & $\begin{array}{l}88 \\
40\end{array}$ & \\
\hline$U=2320001963$ ODS $02 / 08 / 65$ & 506,550 & 0.9791 & 8.12352 & 46. $>$ & 13 & & 323 & 4103 & 64 & 64 & \\
\hline $\begin{array}{llll}232 & 000 & 1963 & 0 D S\end{array}$ & & $\begin{array}{l}0,9967 \\
0.9683\end{array}$ & $\begin{array}{l}8.09 \\
8.05\end{array}$ & & & 839 & & & $\begin{array}{l}64 \\
53\end{array}$ & $\begin{array}{l}64 \\
53\end{array}$ & \\
\hline $\begin{array}{l}20208065 \\
02 / 00165 \\
02165\end{array}$ & & $0.98 \mathrm{~g} \quad \mathrm{C}$ & $\begin{array}{l}8,02814 \\
7.99672\end{array}$ & 23.4 & . & 11574 & & & 43 & 43 & \\
\hline 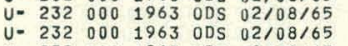 & $\begin{array}{l}51,550 \\
512,550\end{array}$ & & 7:965449 & $40,0>2>$ & 113. & $\begin{array}{l}11603 \\
11557\end{array}$ & & & & 86 & \\
\hline & $51355^{\circ}$ & & $7: 90$ & & & & & & & & \\
\hline 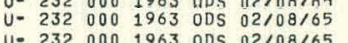 & $\begin{array}{l}515.550 \\
516.550\end{array}$ & 01 & $\begin{array}{l}7.84237 \\
7.81233\end{array}$ & $\begin{array}{l}-22,7 \\
-0.5\end{array}$ & $\begin{array}{l}-63 \\
-26 \\
-26\end{array}$ & & & & 51 & 51 & \\
\hline 19 & 516. & & & & & 233 & & & 57 & 57 & \\
\hline 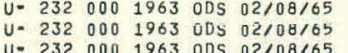 & $\begin{array}{l}517.550 \\
518.550\end{array}-50$ & $\begin{array}{l}0,986 \\
0,986\end{array}$ & 7.78 & $\begin{array}{l}26 . \\
30 .\end{array}$ & & $\begin{array}{l}131 \\
146\end{array}$ & & & $\begin{array}{l}72 \\
80\end{array}$ & $\begin{array}{l}72 \\
80\end{array}$ & \\
\hline 1963 ODS $02 / 0$ & $\begin{array}{l}519 \\
520\end{array}$ & $0,99 \quad>$ & 7.72 & & $\begin{array}{r}133 \\
44\end{array}$ & & & & & 85 & \\
\hline $\begin{array}{l}963 \text { ons } 02 / 08865 \\
.963 \text { ODS } 02 / 108 / 65\end{array}$ & $\begin{array}{l}521.550 \\
522.520\end{array}-2000$ & 00 & 70603366 & $\begin{array}{l}-8,8 \\
34,5 \\
-3\end{array}$ & $\begin{array}{l}-24 \\
95\end{array}$ & & & & & $\begin{array}{l}55 \\
91 \\
91\end{array}$ & \\
\hline $\begin{array}{ll}5 & 021 \\
5 & 021\end{array}$ & & & $\begin{array}{l}7.60 \\
7.57\end{array}$ & & & & & & 58 & 58 & \\
\hline 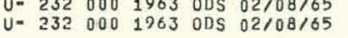 & $\begin{array}{l}525.550 \\
526.550\end{array}$ & $\begin{array}{l}0.9777 \\
0.9954\end{array}$ & $\begin{array}{l}7.55676 \\
7.51812\end{array}$ & 10,16 & & 11060 & & & 然 & 98 & \\
\hline
\end{tabular}




\begin{tabular}{|c|c|c|c|c|c|c|c|c|c|c|c|}
\hline IDENTIFICATION & DELAY & TRANS, & ENERGY & $x=\varepsilon_{E C}$ & 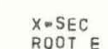 & COPEN & VTS & $\begin{array}{l}F R A C_{1} \\
O E O C T\end{array}$ & $\begin{array}{l}\text { FAST B } \\
\text { OPEN }\end{array}$ & GS & No, PTS \\
\hline 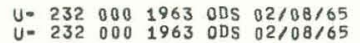 & $\begin{array}{l}527.550 \\
528.550\end{array}$ & $\begin{array}{l}1.0072 \\
0.9998\end{array}$ & $7: 48965$ & $\begin{array}{r}-15.89 \\
0.45\end{array}$ & $\begin{array}{r}-43.48 \\
1.22\end{array}$ & $\begin{array}{l}11045 \\
11195\end{array}$ & $\begin{array}{l}10867 \\
10933\end{array}$ & $\begin{array}{l}0: 4273 \\
0: 4281\end{array}$ & $\begin{array}{l}55 \\
69\end{array}$ & $\begin{array}{l}55 \\
69 \\
69\end{array}$ & \\
\hline $\begin{array}{l}0 \\
0 \\
0 \\
0\end{array}$ & $\begin{array}{l}529.550 \\
530.550\end{array}$ & $\begin{array}{l}0.9940 \\
0.9944\end{array}$ & $\begin{array}{l}7: 43318 \\
7: 0519\end{array}$ & $\begin{array}{l}13.35 \\
12.55\end{array}$ & $\begin{array}{l}36.41 \\
34.16\end{array}$ & $\begin{array}{l}111008 \\
11165\end{array}$ & $\begin{array}{l}1088 \mathrm{E} \\
10844\end{array}$ & $\begin{array}{l}0: 4289 \\
0: 4297\end{array}$ & 995 & 99 & $\begin{array}{c}2 \\
2 \\
2\end{array}$ \\
\hline $\begin{array}{l}0.000 \\
000 \\
0006303\end{array}$ & $\begin{array}{l}531,550 \\
532,550\end{array}$ & $\begin{array}{l}0.6964 \\
0.9858\end{array}$ & $\begin{array}{l}7,37735 \\
7: 34967\end{array}$ & $\begin{array}{l}8,02 \\
31,60\end{array}$ & $\begin{array}{l}21,78 \\
85.68\end{array}$ & $\begin{array}{l}11107 \\
11084\end{array}-\mathrm{V} \mathrm{l}$ & 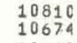 & $\begin{array}{l}0.4306 \\
0,4314\end{array}-200$ & $\begin{array}{l}54 \\
65\end{array}$ & $\begin{array}{l}54 \\
65\end{array}$ & \\
\hline 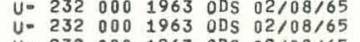 & $\begin{array}{l}533.550 \\
534.520\end{array}-100$ & $\begin{array}{l}0.9654 \\
0.9937\end{array}$ & $\begin{array}{r}7: 32215 \\
7: 29478\end{array}$ & $\begin{array}{l}78,00 \\
14,04\end{array}$ & $\begin{array}{r}211,07 \\
37,93\end{array}$ & $\begin{array}{l}10800 \\
10911\end{array}-\mathrm{V} \mathrm{V}$ & $\begin{array}{l}10445 \\
10590\end{array}$ & $\begin{array}{l}0,4322 \\
0,4330\end{array}-2$ & $\begin{array}{l}112 \\
110\end{array}$ & $\begin{array}{l}112 \\
110\end{array}$ & \\
\hline 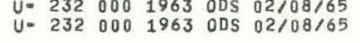 & $\begin{array}{l}5355.550 \\
536.550\end{array}$ & $\begin{array}{l}0.951 \\
1.0121\end{array}$ & $\begin{array}{l}7,2656 \\
7.24050\end{array}$ & $\begin{array}{l}10.89 \\
-26,75\end{array}$ & $\begin{array}{rl}29: 36 & .71: 97\end{array}$ & $\begin{array}{l}10978 \\
10843\end{array}$ & $\begin{array}{l}10671 \\
10720\end{array}$ & $\begin{array}{l}0.4338 \\
0.4346\end{array}$ & $\begin{array}{l}61 \\
92\end{array}$ & $\begin{array}{l}61 \\
92\end{array}$ & \\
\hline $232000 \quad 196300 S \quad 02 / 08 / 65$ & 537,550 & 0.9844 & 7.21358 & 34,92 & 93,80 & 11011 & 10587 & 0.4354 & 42 & 42 & \\
\hline 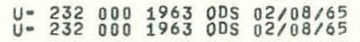 & $\begin{array}{l}538,550 \\
539,550\end{array}$ & $\begin{array}{l}0,9776 \\
1 ; 023\end{array}$ & $7: 186820$ & $\begin{array}{l}50: 12 \\
-5: 14\end{array}$ & $\begin{array}{l}134: 37 \\
-137\end{array}$ & $\begin{array}{l}10873 \\
10944\end{array}$ & $\begin{array}{l}10383 \\
10715\end{array}$ & $\begin{array}{l}0: 4362 \\
0: 4370\end{array}$ & $\begin{array}{l}72 \\
40\end{array}$ & $\begin{array}{l}72 \\
40\end{array}$ & \\
\hline 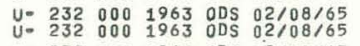 & $\begin{array}{l}540,550 \\
541,550\end{array}$ & $\begin{array}{l}0.9796 \\
1 ; 082\end{array}$ & $7: 137373$ & $\begin{array}{l}45,74 \\
-18: 10\end{array}$ & $\begin{array}{l}122,17 \\
-48\end{array}$ & $\begin{array}{l}10963 \\
10697\end{array}$ & ${ }_{10534}^{1049}$ & $\begin{array}{l}0 ; 4378 \\
0 ; 438\end{array}$ & $\begin{array}{r}72 \\
106\end{array}$ & $\begin{array}{c}72 \\
106\end{array}$ & \\
\hline 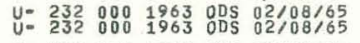 & $\begin{array}{l}542,550 \\
544: 800\end{array}$ & 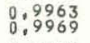 & $\begin{array}{l}7: 08224 \\
7: 0287\end{array}$ & $\begin{array}{l}8,21 \\
6 ; 82\end{array}$ & $\begin{array}{l}21.85 \\
18: 07\end{array}$ & $\begin{array}{l}10828 \\
40057\end{array}$ & 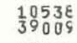 & $\begin{array}{l}0: 4395 \\
0: 4413\end{array}$ & $22^{5 \frac{1}{3}}$ & $\begin{aligned} 28 \frac{1}{3} \\
2\end{aligned}$ & \\
\hline 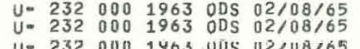 & $\begin{array}{l}548,300 \\
552 ; 300\end{array}-10$ & $\begin{array}{l}0,9832 \\
0,9785\end{array}$ & $\begin{array}{l}6.93350 \\
6: 83343\end{array}$ & $\begin{array}{l}37,49 \\
48 ; 15\end{array}$ & $\begin{array}{r}98,73 \\
125,86\end{array}$ & $\begin{array}{l}35548 \\
50445\end{array}-12 \mathrm{r}-2$ & $\begin{array}{l}335752 \\
48216\end{array}-100$ & $\begin{array}{l}0 \\
0 ; 4474 \\
0,447\end{array}$ & $\begin{array}{l}200 \\
311\end{array}$ & $\begin{array}{l}200 \\
311\end{array}$ & \\
\hline & & & & & & & & & & & \\
\hline 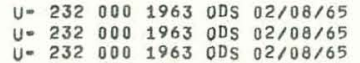 & $\begin{array}{l}560.550 \\
564.800\end{array}$ & $\begin{array}{l}0.9917 \\
0.9983 \\
0.9712\end{array}$ & $\begin{array}{l}6.63376 \\
6.53330 \\
6.534141\end{array}$ & $\begin{array}{l}18.52 \\
23.80\end{array}$ & $\begin{array}{r}47,70 \\
60.84 \\
64.86\end{array}$ & $\begin{array}{l}41520 \\
4006\end{array}$ & 126 & & $\begin{array}{l}255 \\
366\end{array}$ & $\begin{array}{l}236 \\
236\end{array}$ & \\
\hline 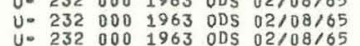 & $\begin{array}{l}572,050 \\
573050\end{array}$ & $\begin{array}{ll}0.9571 \\
0.947 \\
0.948\end{array}$ & 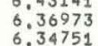 & $\begin{aligned} 64,85 \\
97,25 \\
114,20\end{aligned}$ & $\begin{array}{l}164.46 \\
245.45 \\
2,45\end{array}$ & $\begin{array}{l}48009 \\
10201 \\
10206\end{array}-\mathrm{l}$ & $\begin{array}{r}46302 \\
9537 \\
9556\end{array}$ & & $\begin{array}{c}348 \\
61 \\
60\end{array}$ & $\begin{array}{c}348 \\
61 \\
61\end{array}$ & \\
\hline $022108 / 165$ & 574.050 & 0.09709 & & 65.57 & & & & & 80 & 80 & \\
\hline $\begin{array}{l}1963 \text { ODS } 02 / 08655 \\
1963 \text { oDS } 02 / 10865 \\
1963005\end{array}$ & $\begin{array}{l}575.050 \\
576.050\end{array}$ & $\begin{array}{l}0.940 \\
0,9402 \\
0.9402\end{array}$ & . 283457 & $\begin{array}{l}137,19 \\
136,64\end{array}-\mathrm{V}$ & 340.47 & $\begin{array}{l}1027 \\
10035\end{array}$ & $\begin{array}{l}9295 \\
9216 \\
9216\end{array}-10$ & & 15 & $\begin{array}{l}06 \\
15 \\
\end{array}$ & \\
\hline 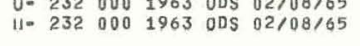 & $\begin{array}{l}978.050 \\
578,050\end{array}$ & $\begin{array}{l}0,0200 \\
0,920\end{array}$ & $\begin{array}{l}6.23816 \\
6.2382\end{array}$ & $\begin{array}{l}113.42 \\
184.84\end{array}$ & $\begin{array}{l}408.87 \\
461.67\end{array}$ & $\begin{array}{l}10036 \\
9989\end{array}$ & $\begin{array}{l}8106 \\
8976\end{array}$ & $\begin{array}{l}0.4674 \\
0.4682\end{array}$ & $\begin{array}{c}133 \\
61\end{array}$ & 11 & \\
\hline $\begin{array}{l}02 / 08 / 65 \\
02 / 8165\end{array}$ & $\begin{array}{l}579,050 \\
580\end{array}$ & 0.8984 & 1065 & 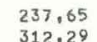 & 2,53 & $\begin{array}{l}10041 \\
10198\end{array}$ & $\begin{array}{l}8811 \\
8652 \\
865-30\end{array}$ & 要 & & ${ }^{43}$ & \\
\hline $\begin{array}{l}-10165 \\
02120165 \\
02 / 0165\end{array}$ & $\begin{array}{l}581: \\
582:\end{array}$ & $\begin{array}{l}0.858 \\
0.809\end{array}$ & 3 & $337:$ & & 10113 & 8485 & & & & \\
\hline 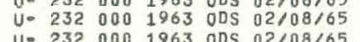 & $\begin{array}{l}583.050 \\
584050\end{array}$ & $\begin{array}{l}0.0122 \\
0.741 \\
0.645\end{array}$ & $\begin{array}{l}0.1375 \\
6.13165\end{array}$ & 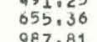 & $\begin{array}{l}121030 \\
1622.82\end{array}$ & $\begin{array}{ll}9928 \\
9958\end{array}-173$ & $\begin{array}{l}7237 \\
6236 \\
6200\end{array}$ & & $\begin{array}{lll}84 \\
80\end{array}$ & 年 & \\
\hline $\begin{array}{l}19630050202 / 18 / 65 \\
1963 \text { ons } 02208 / 65\end{array}$ & $\begin{array}{l}584.050 \\
585.050\end{array}$ & $\begin{array}{l}0,645 \\
0,5103\end{array}$ & $\begin{array}{l}6.11067 \\
6.00980\end{array}$ & $\begin{array}{r}987,81 \\
1491,30\end{array}$ & $\begin{array}{l}2441.84 \\
3680.15\end{array}$ & 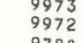 & $\begin{array}{l}6235 \\
4971 \\
4971\end{array}$ & 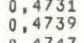 & $\begin{array}{c}94 \\
110\end{array}$ & $\begin{array}{l}44 \\
0\end{array}$ & \\
\hline $\begin{array}{l}02208 / 155 \\
02 / 08 / 65\end{array}$ & $\begin{array}{l}586,050 \\
587: 050\end{array}$ & $\begin{array}{l}0.4068 \\
0: 2530\end{array}$ & 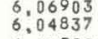 & 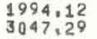 & $\begin{array}{l}4912,60 \\
7494,33\end{array}-100$ & $\begin{array}{l}9780 \\
9900\end{array}-100$ & $\begin{array}{l}3886 \\
2446\end{array}-10$ & $\begin{array}{l}4747 \\
4755\end{array}$ & $\begin{array}{l}57 \\
73\end{array}$ & $\begin{array}{l}57 \\
73\end{array}$ & \\
\hline 2320001963 ODS $02 / 08 / 65$ & 588.050 & 0.1534 & 6.02782 & 4156,46 & 10204.78 & & 1481 & 3 & 83 & 83 & \\
\hline 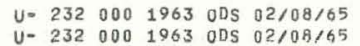 & $\begin{array}{l}589.050 \\
590\end{array}$ & 0,0983 & $\begin{array}{l}6.00737 \\
5.98702\end{array}$ & $\begin{array}{l}5142,47 \\
5263,29\end{array}$ & & & 943 & 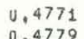 & 98 & 98 & \\
\hline 1963 oDs 027108165 & $\begin{array}{l}591.050 \\
59.050\end{array}$ & $\begin{array}{l}0.091 \\
0.059 \\
0.137\end{array}$ & $\begin{array}{l}5: 9678 \\
5 ; 8\end{array}$ & 5197,24 & 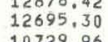 & 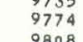 & 919 & 8 & 88 & 列 & \\
\hline $\begin{array}{l}502 / 188 / 55 \\
02 / 08 / 65\end{array}$ & $\begin{array}{l}592,050 \\
593.050\end{array}$ & $\begin{array}{l}0.1374 \\
0.2105\end{array}$ & 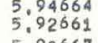 & $\begin{array}{l}4400: 10 \\
3454: 74\end{array}$ & $\begin{array}{r}10729,96 \\
8410,43\end{array}$ & $\begin{array}{l}9808 \\
9850\end{array}-10$ & $\begin{array}{l}1316 \\
2028\end{array}$ & $\begin{array}{l}0.4864 \\
0.404\end{array}$ & 796 & $\begin{array}{l}46 \\
79\end{array}$ & \\
\hline $\begin{array}{l}0 \\
02 / 08865 \\
02 / 08 / 65\end{array}$ & $\begin{array}{l}594: 050 \\
595: 050\end{array}$ & $\begin{array}{l}0.3538 \\
0: 4910\end{array}$ & $\begin{array}{l}55: 90667 \\
5 ; 8683\end{array}$ & $\begin{array}{l}2303,56 \\
1576,80\end{array}$ & $\begin{array}{l}5598,50 \\
3825: 75\end{array}$ & $\begin{array}{l}9758 \\
9889 \\
988020\end{array}$ & $\begin{array}{l}3372 \\
4743 \\
473-100\end{array}$ & $\begin{array}{l}0.4812 \\
0: 4820\end{array}$ & $\begin{array}{l}57 \\
66\end{array}$ & $\begin{array}{l}57 \\
66\end{array}$ & \\
\hline 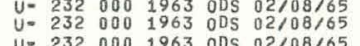 & $\begin{array}{l}596 \% 050 \\
597.050\end{array}$ & $\begin{array}{l}0 \\
0.6352 \\
0.748\end{array}$ & $\begin{array}{l}5: 86710 \\
5: 8746 \\
5\end{array}$ & $\begin{array}{l}1050.99 \\
663.27 \\
6.27\end{array}$ & $\begin{array}{l}2436,71 \\
1652.26\end{array}$ & $\begin{array}{l}9667 \\
9484 \\
945\end{array} \mathrm{l}$ & $\begin{array}{l}5998 \\
6807\end{array}-2$ & $\begin{array}{l}4828 \\
4836\end{array}-136$ & $\frac{41}{3}$ & $\frac{4 \frac{1}{3}}{3}$ & \\
\hline & & & & & & & & & & & \\
\hline $232000196300002 / 08 / 65$ & 600.550 & $\begin{array}{l}0.8432 \\
0.8772\end{array}$ & 77050 & $\begin{array}{l}378,21 \\
330,24\end{array}$ & . & & 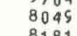 & & 30 & $30,0>030$ & \\
\hline $\begin{array}{l}02108 / 65 \\
02208 / 65 \\
0205\end{array}$ & $\begin{array}{l}602.530 \\
602.550\end{array}$ & 0.8832 & $\begin{array}{l}4420 \\
21219 \\
2219\end{array}-100$ & $\begin{array}{l}275.47 \\
116.97\end{array}$ & $\begin{array}{l}6600 \\
401:\end{array}$ & 9571 & $\frac{6}{1}$ & & $\begin{array}{l}65 \\
73\end{array}$ & 73 & \\
\hline $\begin{array}{l}02108165 \\
021085 \\
0210865\end{array}$ & $\begin{array}{l}604.550 \\
605550\end{array}$ & $\begin{array}{l}0.9171 \\
0.930\end{array}$ & $\begin{array}{l}5: 70327 \\
5.68445\end{array}$ & $\begin{array}{l}191,83 \\
156,04\end{array}$ & $\begin{array}{l}356 \\
372 \\
372\end{array}$ & $\begin{array}{l}9491 \\
9547\end{array}-1$ & $\begin{array}{l}850 द \\
8601 \\
8601\end{array}$ & & $\begin{array}{l}81 \\
83\end{array}$ & $8 \frac{1}{83}$ & \\
\hline $\begin{array}{l}02708 \\
02108\end{array}$ & $\begin{array}{l}606.550 \\
60.550\end{array}$ & $\begin{array}{l}0 \\
0,9458 \\
0.937\end{array}$ & 5.6 & $\begin{array}{l}123,44 \\
1124,438\end{array}$ & 29 & & 8717 & & & 40 & \\
\hline $\begin{array}{l}1963 \text { OUS } 02 / 0865 \\
1963 \text { ODS } \\
02 / 08 / 65\end{array}$ & $\begin{array}{l}608,550 \\
600\end{array}$ & $\begin{array}{l}0: 9370 \\
0: 9410\end{array}$ & $\begin{array}{l}5,62855 \\
5 ; 6280\end{array}$ & $\begin{array}{l}1144,78 \\
134,76\end{array}$ & 319 & 9412 & $\begin{array}{l}8751 \\
8651\end{array}$ & 9929 & $\begin{array}{l}76 \\
70\end{array}$ & 70 & \\
\hline $02 / 081$ & 609,550 & 0.93 & 5,61009 & 143,2 & 339.33 & 9408 & 8615 & 1937 & 62 & 62 & \\
\hline $\begin{array}{l}02 / 08,65 \\
02 / 00 / 65 \\
0200\end{array}$ & $\begin{array}{l}610.550 \\
613 ; 550\end{array}$ & $\begin{array}{l}0: 971 \\
0: 962 \\
0.992\end{array}$ & $\begin{array}{l}5: 5973 \\
5: 53718 \\
5\end{array}$ & - & 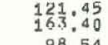 & $\begin{array}{l}9214 \\
4648 \\
6297\end{array}$ & $\begin{array}{l}88794 \\
44006 \\
4006\end{array}$ & 50 & $3^{8}$ & $\begin{array}{l}855 \\
352 \\
525\end{array}$ & \\
\hline $\begin{array}{l}0202108 / 155 \\
02 / 08 / 65\end{array}$ & $\begin{array}{l}618,800 \\
624.550\end{array}$ & $\begin{array}{l}0: 981 \\
0: 9758\end{array}$ & $\begin{array}{l}5: 44462 \\
5.34385\end{array}$ & $\begin{array}{l}\begin{array}{l}42,23 \\
54,38\end{array}-13 \\
5\end{array}$ & $\begin{array}{r}98 \\
125 \\
\end{array}$ & $\begin{array}{l}5268 \\
5433\end{array}$ & $\begin{array}{l}50494 \\
51786\end{array}$ & 2 & 620 & $\begin{array}{l}505 \\
620 \\
620\end{array}-2$ & \\
\hline $\begin{array}{l}02108 / 65 \\
02 / 08 / 65\end{array}$ & $\begin{array}{l}\begin{array}{r}630,550 \\
636,590\end{array} \\
\end{array}$ & $\begin{array}{l}0: 977 \\
0,9823\end{array}$ & $\begin{array}{l}5.242 \\
5.144\end{array}$ & $\begin{array}{l}47,7 \\
39 ;\end{array}$ & $\begin{array}{r}109 \\
89\end{array}$ & $\begin{array}{l}58390 \\
52973\end{array}$ & $\begin{array}{l}51516 \\
50830\end{array}$ & & 10 & $0_{0}^{2}$ & \\
\hline & & & & $\begin{array}{l}42, \\
26,\end{array}$ & & & & & 994 & $\begin{array}{l}107 \\
98\end{array}$ & \\
\hline & & 0.989 & & 23. & & & & & & & \\
\hline 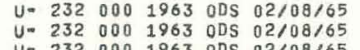 & $\begin{array}{l}688,880 \\
705,050\end{array}$ & $\begin{array}{l}0.993 \\
0: 9861\end{array}$ & $\begin{array}{l}4,3 \\
4: 1\end{array}$ & $\begin{array}{l}2,6 \\
31,0\end{array}$ & & $\begin{array}{l}115555 \\
118047\end{array}$ & $\begin{array}{l}111 \\
113\end{array}$ & & $\begin{array}{l}1110 \\
1380\end{array}$ & $\begin{array}{l}1110 \\
1380\end{array}$ & \\
\hline 0216 & $\begin{array}{l}722 . \\
741 .\end{array}$ & & & $\begin{array}{l}6.6 \\
24:\end{array}$ & & & & & & & \\
\hline 1963 o & $\begin{array}{l}761,800 \\
784,050\end{array}$ & $\begin{array}{l}0,99 \\
0,99\end{array}$ & & 21. & & $\begin{array}{l}137862 \\
134987\end{array}$ & & & & & \\
\hline $\begin{array}{llll}0 & 000 & 1963 & 00 \\
0 & 1963 & 0 & 0\end{array}$ & & & & & & & & & & & \\
\hline $000196300502 / 08 / 65$ & 896,840 & 0.9887 & 2.59178 & & & & & & & & \\
\hline
\end{tabular}




\section{Appendix B}

$$
\text { A Cross Section Compilation for }{ }^{232} \mathrm{U}
$$

This cross section compilation was taken from the data listed in Appendix A. In general, the thick sample data were used to obtain cross sections in the valleys and the thin sample data were used to give the peak cross sections. The data points shown in Figs. 1 and 2 were plotted from this compilation. These data include instrumentai resolution and Doppler effects. The multilevel fits shown in Figs. 1 and 2 were made to describe these data by using the inverse sample thickness of 2217 barms/atom when doing the instrumental resolution broadening. A debye temperature of $334^{\circ} \mathrm{C}$ was used for the Doppler broadening. 


\begin{tabular}{|c|c|c|c|c|c|c|c|}
\hline $\begin{array}{l}10 \\
1954 \\
1954 \\
1954 \\
1954 \\
1954 \\
1954 \\
1954 \\
1954 \\
1954 \\
1954\end{array}$ & $\begin{array}{l}\text { ENERGY } \\
\text { (EV) } \\
0,01006 \\
0,01055 \\
0,01108 \\
0.01164 \\
0,01223 \\
0,01284 \\
0,01347 \\
0,01416 \\
0,01487 \\
0,01560\end{array}$ & $\begin{array}{c}\text { SIGHA } \\
\text { (BARNS) } \\
265,97 \\
237,06 \\
237,51 \\
239,94 \\
226,92 \\
222,64 \\
216,12 \\
211,75 \\
204,79 \\
203,19\end{array}$ & $\begin{array}{r}\text { SIGMA } \\
\text { ROOT E } \\
26.67 \\
24.35 \\
25.00 \\
25,89 \\
25.10 \\
25.23 \\
25.09 \\
25.19 \\
24.98 \\
25.38\end{array}$ & $\begin{array}{c}1 D \\
1954 \\
1954 \\
1954 \\
1954 \\
1954 \\
1954 \\
1954 \\
1954 \\
1954 \\
1954\end{array}$ & $\begin{array}{l}\text { ENERGY } \\
\text { (EV) } \\
0.11541 \\
0.12127 \\
0.12708 \\
0.13332 \\
0.14004 \\
0.14727 \\
0.15441 \\
0.16208 \\
0.17033 \\
0.17839\end{array}$ & $\begin{array}{r}\text { SIGMA } \\
\text { (BARNS) } \\
69.88 \\
68.40 \\
66.64 \\
64.03 \\
60.82 \\
59.80 \\
58.62 \\
57.81 \\
56.18 \\
53.51\end{array}$ & $\begin{array}{r}\text { SIGMA } \\
\text { ROOT E } \\
23.74 \\
23.82 \\
23.76 \\
23,38 \\
22.76 \\
22.95 \\
23.03 \\
23.27 \\
23.19 \\
22.60\end{array}$ \\
\hline $\begin{array}{l}1954 \\
1954 \\
1954 \\
1954 \\
1954 \\
1954 \\
1954 \\
1954 \\
1954 \\
1954\end{array}$ & $\begin{array}{l}0.01636 \\
0.01718 \\
0.01806 \\
0.01898 \\
0.01991 \\
0,02088 \\
0.02192 \\
0.02304 \\
0.02421 \\
0.02542\end{array}$ & $\begin{array}{l}195,63 \\
196: 52 \\
193,46 \\
189,33 \\
187,77 \\
175,24 \\
172,32 \\
171,51 \\
167,32 \\
164,11\end{array}$ & $\begin{array}{l}25.03 \\
25.76 \\
26.00 \\
26.09 \\
26.50 \\
25.32 \\
25.51 \\
26.03 \\
26.03 \\
26.17\end{array}$ & $\begin{array}{l}1954 \\
1954 \\
1954 \\
1954 \\
1954 \\
1954 \\
1954 \\
1954 \\
1954 \\
1954\end{array}$ & $\begin{array}{l}0.18704 \\
0.19633 \\
0.20633 \\
0.21712 \\
0.24009 \\
0.25226 \\
0.26538 \\
0.27792 \\
0.29137 \\
0.30583\end{array}$ & $\begin{array}{l}52.01 \\
48.65 \\
49.27 \\
45.85 \\
43.69 \\
43.63 \\
42.59 \\
40.43 \\
37.10 \\
40.41\end{array}$ & $\begin{array}{l}22.50 \\
21.55 \\
22.38 \\
21.36 \\
21.41 \\
21.91 \\
21.94 \\
21.31 \\
20.03 \\
22.35\end{array}$ \\
\hline $\begin{array}{l}1954 \\
1954 \\
1954 \\
1954 \\
1954 \\
1954 \\
1954 \\
1954 \\
1954 \\
1954\end{array}$ & $\begin{array}{l}0.02668 \\
0.02798 \\
0.02937 \\
0.03088 \\
0.03244 \\
0.03405 \\
0.03578 \\
0.03757 \\
0.03941 \\
0.04139\end{array}$ & $\begin{array}{l}157,85 \\
155,37 \\
148,99 \\
146,89 \\
141,07 \\
139,04 \\
135,64 \\
131,44 \\
128,20 \\
125,12\end{array}$ & $\begin{array}{l}25.78 \\
25.99 \\
25.54 \\
25.81 \\
25.41 \\
25.66 \\
25.66 \\
25.48 \\
25.45 \\
25.46\end{array}$ & $\begin{array}{l}1954 \\
1954 \\
1954 \\
1954 \\
1954 \\
1954 \\
1954 \\
1954 \\
1954 \\
1954\end{array}$ & $\begin{array}{l}0,32138 \\
0,33816 \\
0,35394 \\
0,37085 \\
0,391770 \\
0,41142 \\
0,42954 \\
0.45223 \\
0,47676 \\
0,49942 .\end{array}$ & $\begin{array}{l}36.69 \\
33.01 \\
32.81 \\
34.91 \\
30.58 \\
32.56 \\
27.11 \\
29.35 \\
26.58 \\
29.16\end{array}$ & $\begin{array}{l}20.80 \\
19.19 \\
19.52 \\
21.26 \\
19.14 \\
20.88 \\
17.77 \\
19.74 \\
18.35 \\
20.61\end{array}$ \\
\hline $\begin{array}{l}1954 \\
1954 \\
1954 \\
1954 \\
1954 \\
1954 \\
1954 \\
1954 \\
1954 \\
1954\end{array}$ & $\begin{array}{l}0.04342 \\
0.04561 \\
0.04796 \\
0.05037 \\
0.05284 \\
0.05549 \\
0.05835 \\
0.06126 \\
0.06422 \\
0.06740\end{array}$ & $\begin{array}{r}122,04 \\
119,70 \\
115,29 \\
112,09 \\
109,48 \\
105,20 \\
102,43 \\
99,54 \\
96,50 \\
93,83\end{array}$ & $\begin{array}{l}25.43 \\
25.56 \\
25.25 \\
25.16 \\
25.17 \\
24.78 \\
24.74 \\
24.64 \\
24.45 \\
24.36\end{array}$ & $\begin{array}{l}1954 \\
1954 \\
1954 \\
1954 \\
1954 \\
1954 \\
1954 \\
1954 \\
1954 \\
1954\end{array}$ & $\begin{array}{l}0.52372 \\
0.54985 \\
0.57798 \\
0.60833 \\
0.63549 \\
0.66450 \\
0.70202 \\
0.73577 \\
0.77200 \\
0.81099\end{array}$ & $\begin{array}{l}25.10 \\
25.41 \\
27.78 \\
25.24 \\
24.51 \\
26.01 \\
26.48 \\
20.98 \\
21.96 \\
23.60\end{array}$ & $\begin{array}{l}18,17 \\
18.84 \\
21,12 \\
19.69 \\
19.54 \\
21,20 \\
22.18 \\
17.99 \\
19.29 \\
21,25\end{array}$ \\
\hline $\begin{array}{l}1954 \\
1954 \\
1954 \\
1954 \\
1954 \\
1954 \\
1954 \\
1954 \\
1954 \\
1954\end{array}$ & $\begin{array}{l}0.07082 \\
0.07428 \\
0.07800 \\
0.08201 \\
0.08606 \\
0.09041 \\
0.09510 \\
0.09982 \\
0.10489 \\
0.10996\end{array}$ & $\begin{array}{l}91,29 \\
89,36 \\
86,73 \\
84,02 \\
80,56 \\
79,59 \\
76,62 \\
76,55 \\
72,68 \\
71,82\end{array}$ & $\begin{array}{l}24.29 \\
24.36 \\
24.22 \\
24.06 \\
23.63 \\
23.93 \\
23.63 \\
24.19 \\
23.54 \\
23.82\end{array}$ & $\begin{array}{l}1954 \\
1954 \\
1954 \\
1954 \\
1897 \\
1897 \\
1897 \\
1897 \\
1960 \\
1897\end{array}$ & $\begin{array}{l}0.05300 \\
0.89837 \\
0.93732 \\
0.97885 \\
1.17485 \\
1.37520 \\
1.57539 \\
2.38130 \\
2.41200 \\
2.58112\end{array}$ & $\begin{array}{l}23.31 \\
21.52 \\
21.42 \\
20.26 \\
21.28 \\
21.51 \\
21.08 \\
18.97 \\
20.91 \\
17.61\end{array}$ & $\begin{array}{l}21.53 \\
20.40 \\
20.73 \\
20.05 \\
23.06 \\
25.22 \\
26.46 \\
29.27 \\
32.48 \\
28.29\end{array}$ \\
\hline
\end{tabular}




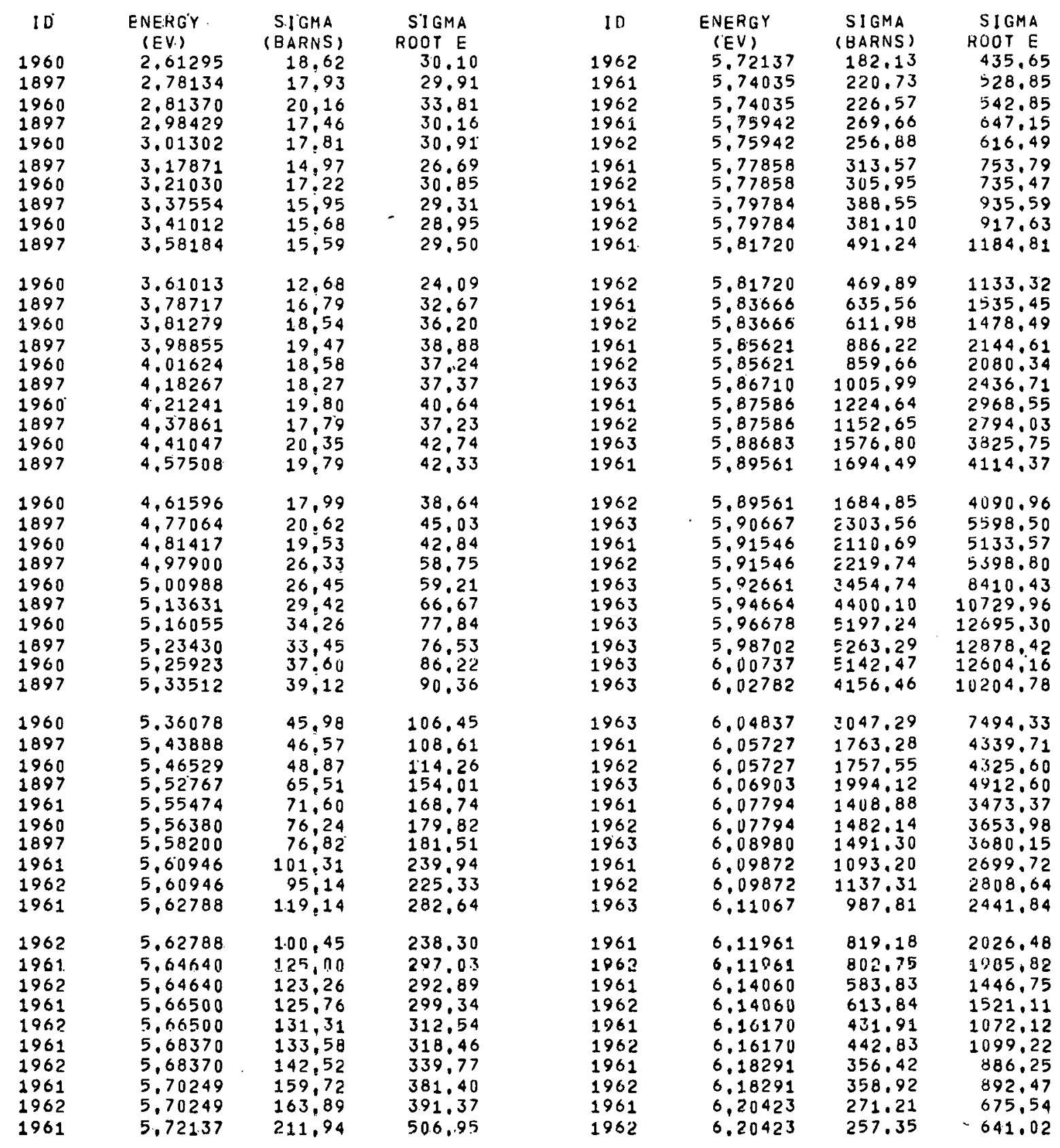




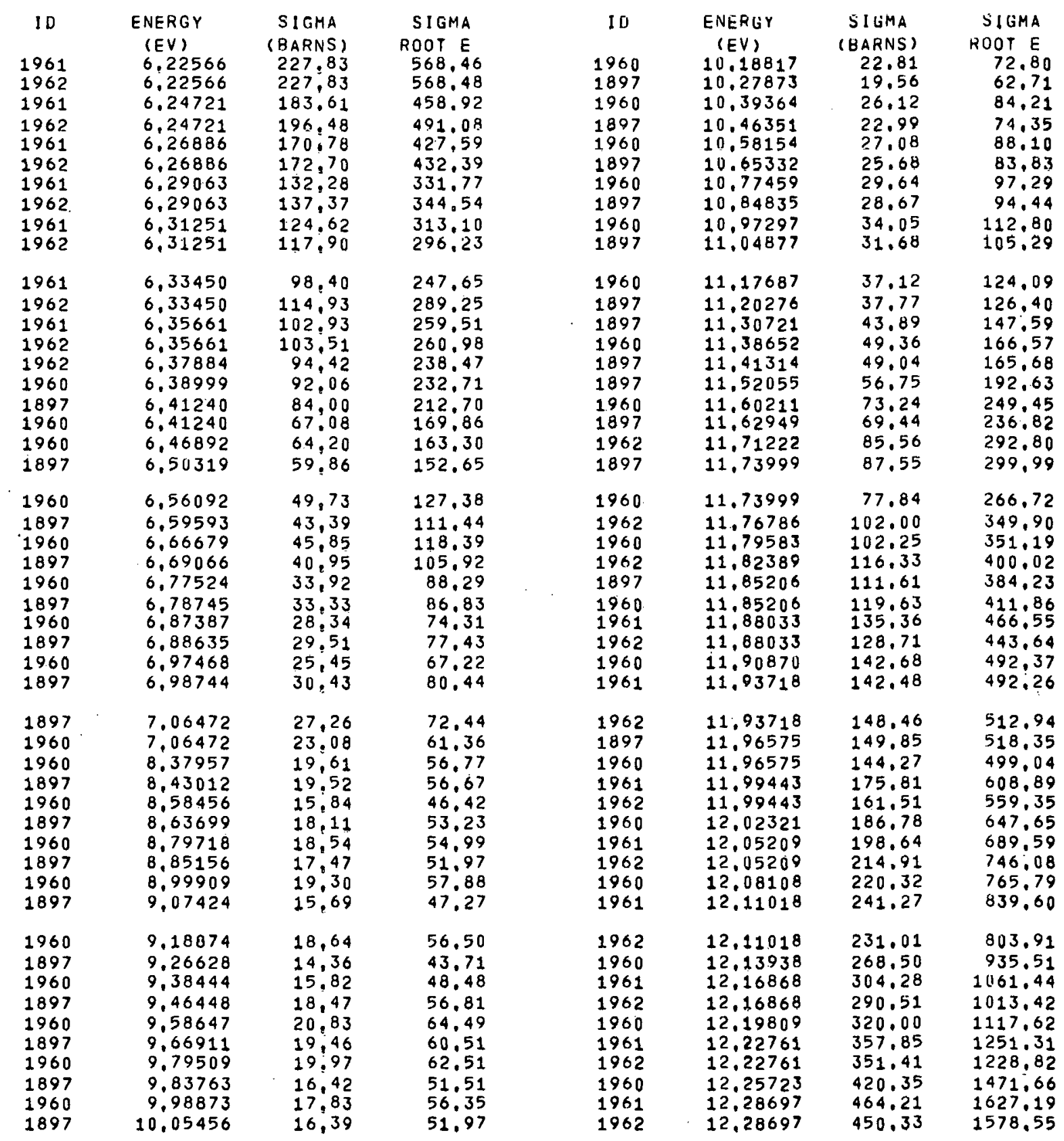




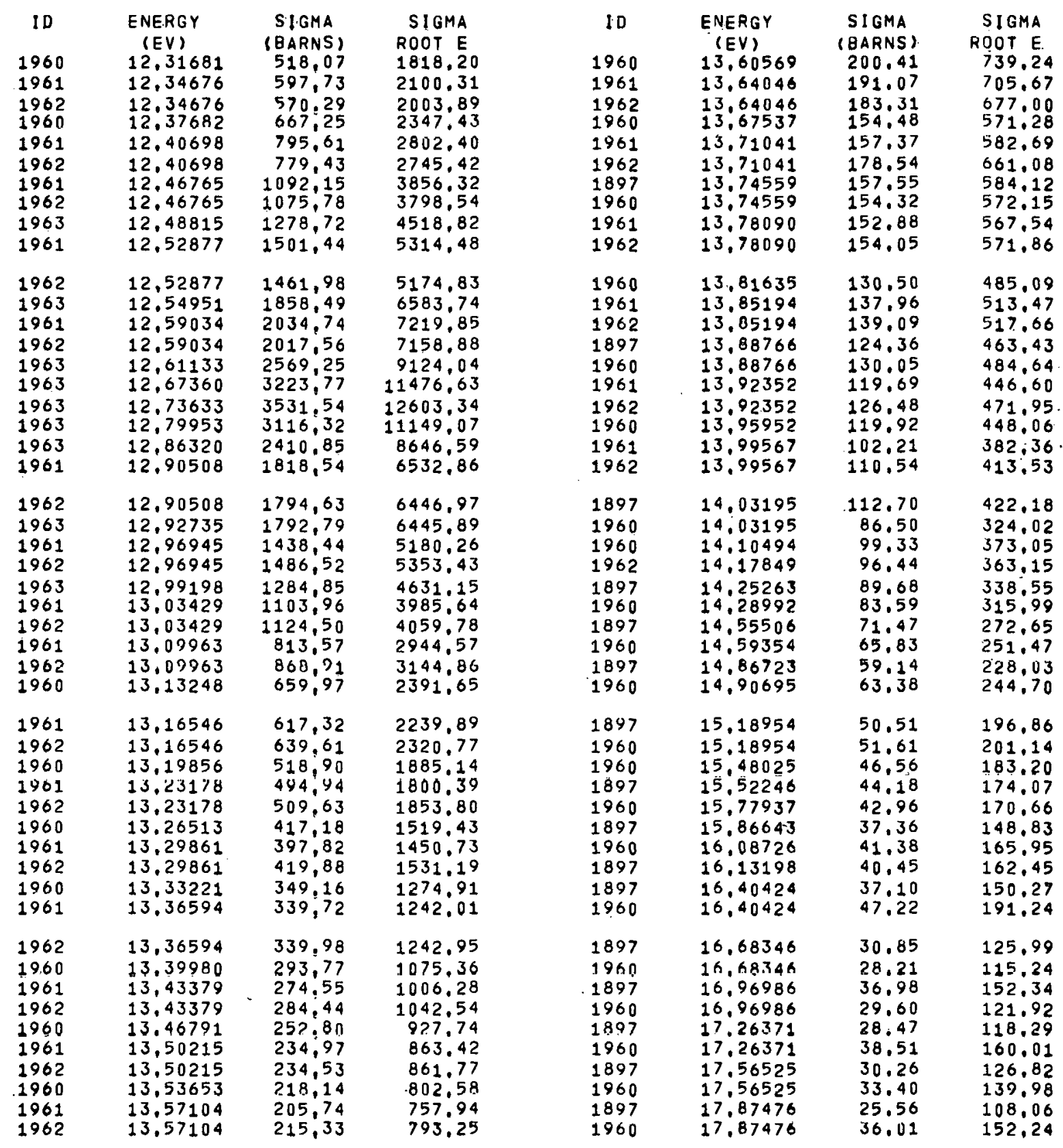




\begin{tabular}{|c|c|c|c|c|c|c|c|}
\hline $\begin{array}{l}10 \\
1897 \\
1960 \\
1897 \\
1960 \\
1897 \\
1960 \\
1897 \\
1960 \\
1960 \\
1897\end{array}$ & $\begin{array}{l}\text { ENERGY } \\
\text { (EV) } \\
18,19253 \\
18.19253 \\
18.51885 \\
18,51885 \\
18,74130 \\
18,79753 \\
19,08256 \\
19,08256 \\
19.37411 \\
19.43322\end{array}$ & $\begin{array}{l}\text { SIGMA } \\
\text { (BARNS) } \\
31,97 \\
32,71 \\
34,64 \\
33,38 \\
35,49 \\
38,93 \\
41,31 \\
43,66 \\
39,17 \\
48,34\end{array}$ & $\begin{array}{l}\text { SIGMA } \\
\text { ROOT E } \\
136.35 \\
139.51 \\
149.08 \\
143.64 \\
153.62 \\
168.79 \\
180.46 \\
190.73 \\
172.41 \\
213.11\end{array}$ & $\begin{array}{l}10 \\
1897 \\
1960 \\
1897 \\
1960 \\
1897 \\
1960 \\
1897 \\
1960 \\
1960 \\
1961\end{array}$ & $\begin{array}{l}\text { ENERGY } \\
\text { (EV) } \\
21.89654 \\
22.03896 \\
22.32799 \\
22.47465 \\
22.62275 \\
22.84766 \\
22,92338 \\
23,07595 \\
23.23004 \\
23,30767\end{array}$ & $\begin{array}{c}\text { SIGMA } \\
\text { (BARNS) } \\
68.51 \\
40.00 \\
28.97 \\
30.91 \\
30.67 \\
34.78 \\
41.97 \\
45.87 \\
67.29 \\
92.29\end{array}$ & $\begin{array}{l}\text { SIGMA } \\
\text { ROOT E } \\
320.57 \\
187.79 \\
136.88 \\
146.55 \\
145.88 \\
166.25 \\
200.97 \\
220.35 \\
324,34 \\
445.56\end{array}$ \\
\hline $\begin{array}{l}1897 \\
1960 \\
1897 \\
1960 \\
1897 \\
1960 \\
1960 \\
1961 \\
1962 \\
1897\end{array}$ & $\begin{array}{l}19,67240 \\
19,67240 \\
19,91602 \\
19,97763 \\
20,16419 \\
20,16419 \\
20,29002 \\
20,35338 \\
20,35338 \\
20,41703\end{array}$ & $\begin{array}{r}45,14 \\
49,30 \\
58,21 \\
62,24 \\
74,66 \\
64,64 \\
83,47 \\
103,10 \\
98,40 \\
106,72\end{array}$ & $\begin{array}{l}200.20 \\
218.65 \\
259.76 \\
278.20 \\
335.24 \\
290.24 \\
375.99 \\
465.14 \\
443,92 \\
482.24\end{array}$ & $\begin{array}{l}1962 \\
1960 \\
1961 \\
1962 \\
1961 \\
1962 \\
1963 \\
1963 \\
1963 \\
1961\end{array}$ & $\begin{array}{l}23,30767 \\
23,38569 \\
23,46410 \\
23,46410 \\
23, .62211 \\
23,62211 \\
23,70243 \\
23,86309 \\
24,02540 \\
24,10582\end{array}$ & $\begin{array}{r}96.00 \\
120.57 \\
183.36 \\
166.81 \\
428.08 \\
394.77 \\
1083.98 \\
1728.82 \\
1047.67 \\
554.79\end{array}$ & $\begin{array}{r}463.47 \\
583.06 \\
888.21 \\
808.01 \\
2080.60 \\
1918.66 \\
5277.38 \\
8445.28 \\
5135,23 \\
2723.89\end{array}$ \\
\hline $\begin{array}{l}1960 \\
1961 \\
1962 \\
1961 \\
1962 \\
1961 \\
1962 \\
1963 \\
1961 \\
1962\end{array}$ & $\begin{array}{l}20,41703 \\
20.48098 \\
20,48098 \\
20,60980 \\
20.60980 \\
20.73983 \\
20,73983 \\
20.80195 \\
20.87109 \\
20.87109\end{array}$ & $\begin{array}{l}103,41 \\
125,70 \\
116,66 \\
145,26 \\
160,69 \\
196,61 \\
190,10 \\
237,56 \\
269,97 \\
266,39\end{array}$ & $\begin{array}{r}467.26 \\
568.89 \\
527.98 \\
659.44 \\
729.49 \\
895.37 \\
865.72 \\
1.083 .50 \\
1233.35 \\
1217.02\end{array}$ & $\begin{array}{l}1962 \\
1961 \\
1962 \\
1960 \\
1961 \\
1962 \\
1960 \\
1897 \\
1960 \\
1897\end{array}$ & $\begin{array}{l}24.10582 . \\
24.27037 \\
24.27037 \\
24.35327 \\
24.43660 \\
24.43660 \\
24.52036 \\
24.85974 \\
24.85974 \\
25.20621\end{array}$ & $\begin{array}{r}593.74 \\
240.24 \\
256.03 \\
138.22 \\
122.31 \\
127.01 \\
84.68 \\
55.58 \\
45.83 \\
38.91\end{array}$ & $\begin{array}{r}2915.14 \\
1183.54 \\
1261.33 \\
682.12 \\
604.60 \\
627.85 \\
419.31 \\
277.14 \\
228.51 \\
195.34\end{array}$ \\
\hline 1963 & 20.93400 & 299.77 & 1371.57 & $\begin{array}{l}1960 \\
1807\end{array}$ & 25.29396 & 31.65 & $159 \cdot 16$ \\
\hline $\begin{array}{l}1901 \\
1962 \\
1963 \\
1961 \\
1962\end{array}$ & $\begin{array}{l}21.00360 \\
21.00360 \\
21.13732 \\
21.13738\end{array}$ & $\begin{array}{l}345,09 \\
354,57 \\
347,08 \\
359,93 \\
374,67\end{array}$ & $\begin{array}{l}1585.20 \\
1624,99 \\
1593.06 \\
1654.78 \\
1722.54\end{array}$ & $\begin{array}{l}1897 \\
1960 \\
1897 \\
1960 \\
1897\end{array}$ & $\begin{array}{l}25.55998 \\
25.64958 \\
25.92125 \\
26.01276 \\
26.29023\end{array}$ & $\begin{array}{l}26.83 \\
24,91 \\
28,43 \\
32,70\end{array}$ & $\begin{array}{l}173.70 \\
135.86 \\
126.85 \\
144.98 \\
167.64\end{array}$ \\
\hline $\begin{array}{l}1963 \\
1961 \\
1962 \\
1963\end{array}$ & $\begin{array}{l}21: 20191 \\
21,27245 \\
21.27245 \\
21.33780\end{array}$ & $\begin{array}{l}342,39 \\
300,03 \\
322,64 \\
199,00\end{array}$ & $\begin{array}{r}1576,56 \\
1383,80 \\
1488,10 \\
919.24\end{array}$ & $\begin{array}{l}1960 \\
1897 \\
1960 \\
1897\end{array}$ & $\begin{array}{l}26.38370 \\
26,66714 \\
26.76264 \\
27.05222\end{array}$ & $\begin{array}{l}26.14 \\
29.52 \\
30.69 \\
46.24\end{array}$ & $\begin{array}{l}134.26 \\
152.46 \\
158.75 \\
240.50\end{array}$ \\
\hline $\begin{array}{l}1961 \\
1962 \\
1963\end{array}$ & $\begin{array}{l}21.40881 \\
21.40881 \\
21.47500\end{array}$ & $\begin{array}{l}204,17 \\
214,30 \\
170,95\end{array}$ & $\begin{array}{l}944.70 \\
991.56 \\
792.20\end{array}$ & $\begin{array}{l}1960 \\
1962 \\
1960\end{array}$ & $\begin{array}{l}27.05222 \\
27.14980 \\
27.24790\end{array}$ & $\begin{array}{l}54.64 \\
63.27 \\
81.77\end{array}$ & $\begin{array}{l}284.21 \\
329.65 \\
426.86\end{array}$ \\
\hline $\begin{array}{l}1960 \\
1961 \\
1962 \\
1963\end{array}$ & $\begin{array}{l}21.47748 \\
21.54649 \\
21.54649 \\
21.61353\end{array}$ & $\begin{array}{l}152,66 \\
131,20 \\
148,96 \\
114,98\end{array}$ & $\begin{array}{l}707.51 \\
609.01 \\
691.44 \\
534.53\end{array}$ & $\begin{array}{l}1961 \\
1962 \\
1960 \\
1961\end{array}$ & $\begin{array}{l}27.34653 \\
27.34653 \\
27.44570 \\
27.54542\end{array}$ & $\begin{array}{r}116.41 \\
98.96 \\
174.23 \\
280.04\end{array}$ & $\begin{array}{r}608.76 \\
517.52 \\
912.78 \\
1469.78\end{array}$ \\
\hline $\begin{array}{l}1960 \\
1962 \\
1960\end{array}$ & $\begin{array}{l}21,61582 \\
21,68549 \\
21,75550\end{array}$ & $\begin{array}{r}114,80 \\
94,84 \\
94,61 \\
65,20\end{array}$ & $\begin{array}{l}440.95 \\
440.57 \\
304.09\end{array}$ & $\begin{array}{l}1901 \\
1962 \\
1963 \\
1961\end{array}$ & $\begin{array}{l}27.54542 \\
27.55278 \\
27.74648\end{array}$ & $\begin{array}{l}257.58 \\
324.77 \\
540.05\end{array}$ & $\begin{array}{l}1351.85 \\
1704.72 \\
2844.71\end{array}$ \\
\hline
\end{tabular}




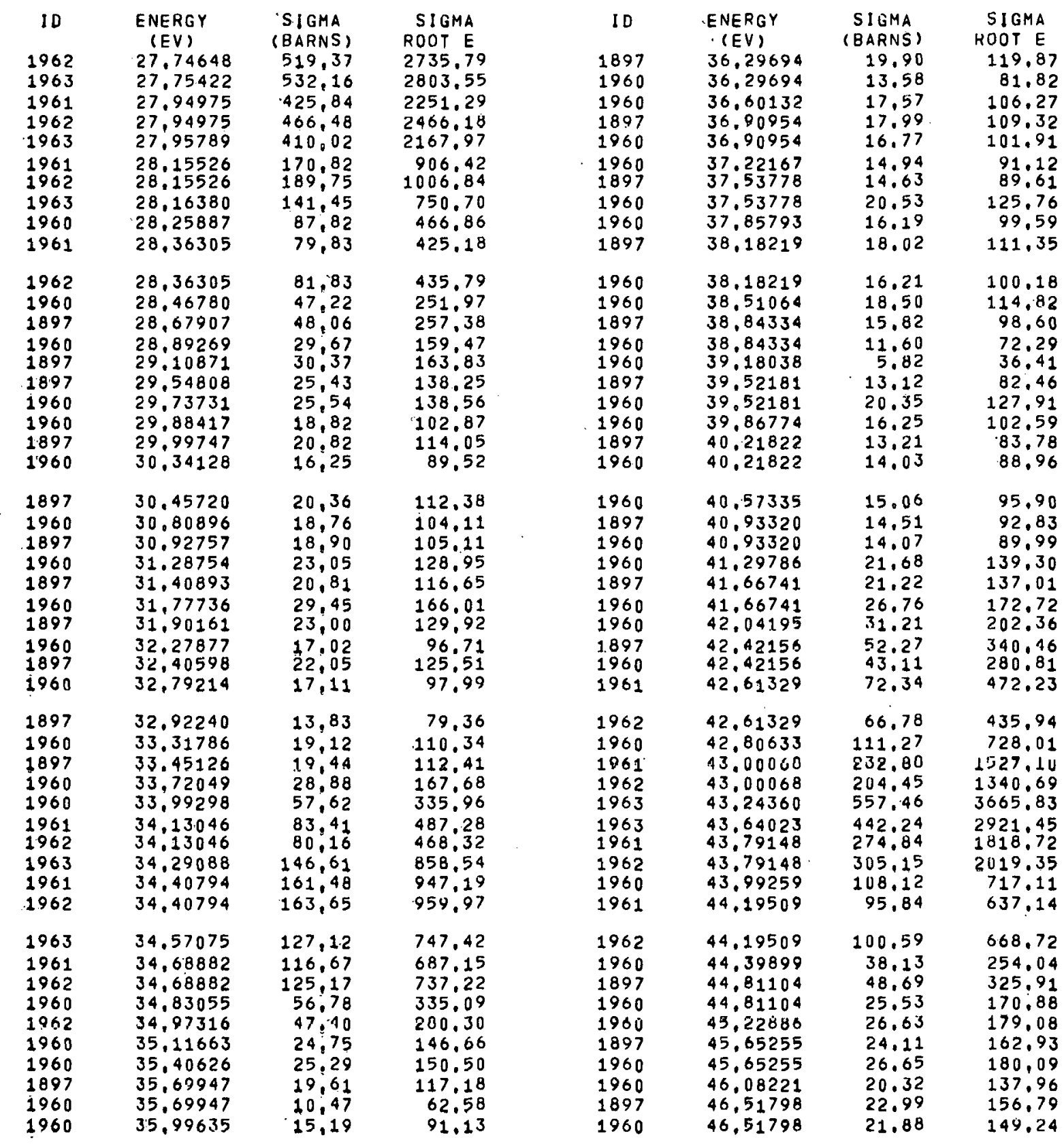




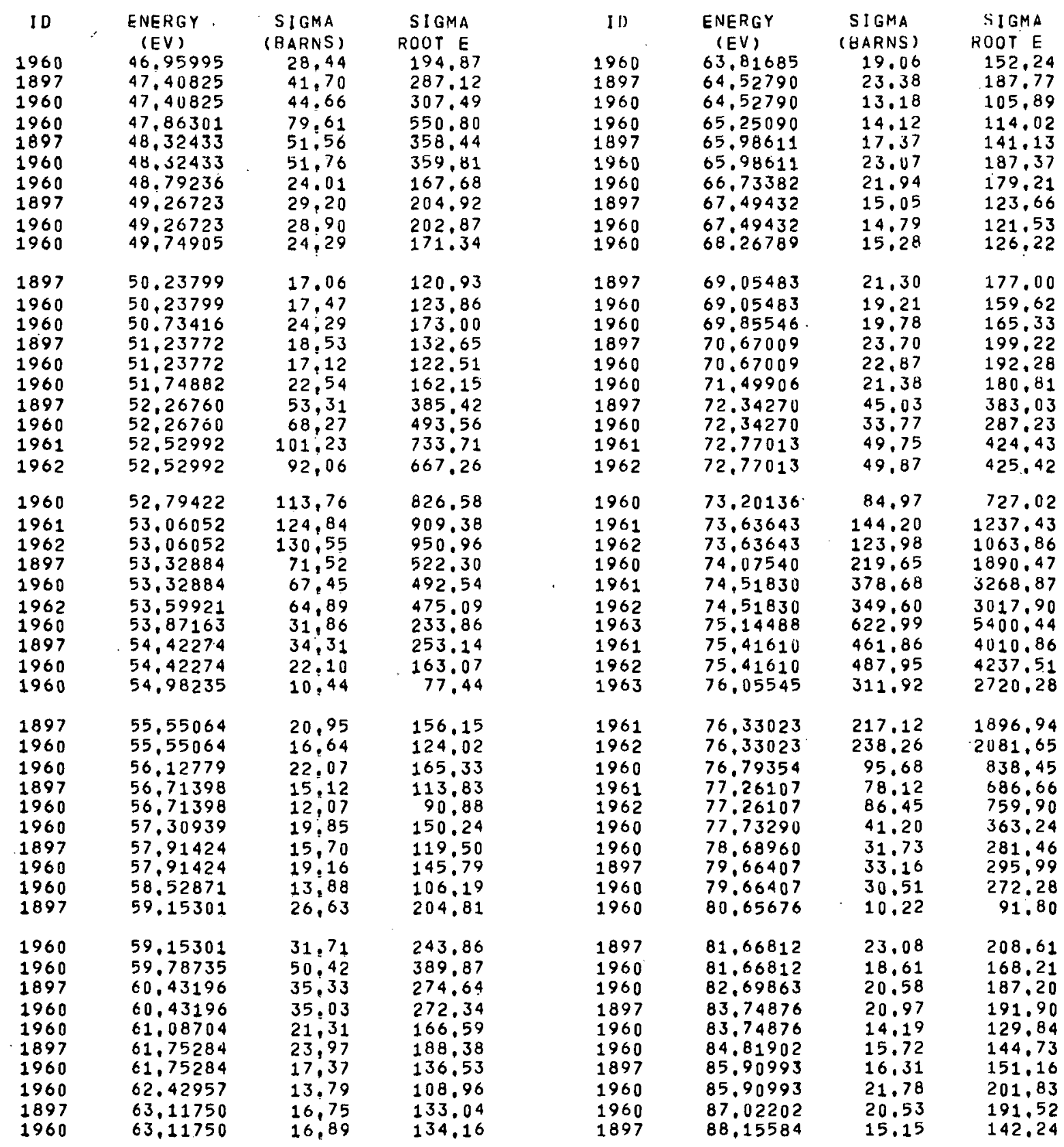




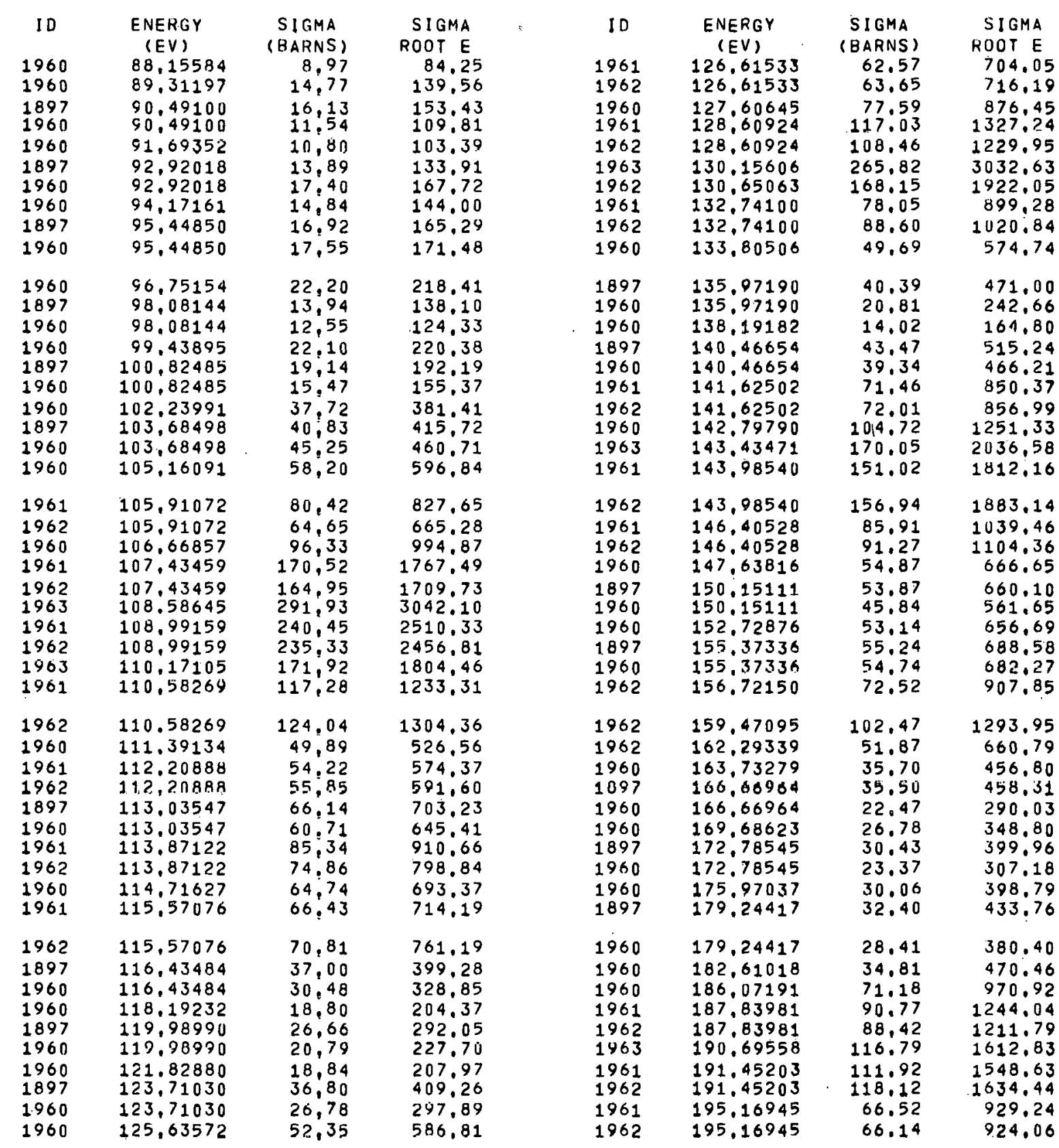




\begin{tabular}{|c|c|c|c|c|c|c|c|}
\hline $\begin{array}{l}10 \\
1960 \\
1897 \\
1960 \\
1960 \\
1697\end{array}$ & $\begin{array}{c}\text { ENERGY } \\
(E V) \\
197.06890 \\
200.95194 \\
200.95194 \\
204.95089 \\
209.07040\end{array}$ & $\begin{array}{c}\text { SIGMA } \\
\text { (BARNS) } \\
37,72 \\
43,83 \\
32,86 \\
27,29 \\
23,77\end{array}$ & $\begin{array}{l}\text { SIGMA } \\
\text { RDOT E } \\
529.52 \\
621.31 \\
465.89 \\
390.68 \\
343.67\end{array}$ & $\begin{array}{l}10 \\
1960 \\
1897 \\
1960 \\
1960 \\
1897\end{array}$ & $\begin{array}{c}\text { ENERGY } \\
\text { (EV) } \\
414.73993 \\
426.67428 \\
426.67428 \\
439.13127 \\
452.14186\end{array}$ & $\begin{array}{c}\text { SIGMA } \\
\text { (BARNS) } \\
32.47 \\
36.42 \\
37.24 \\
41.17 \\
40.89\end{array}$ & $\begin{array}{c}\text { SIGMA } \\
\text { ROOT E } \\
661.34 \\
752.24 \\
769.14 \\
862.65 \\
869.37\end{array}$ \\
\hline $\begin{array}{l}1960 \\
1960 \\
1897 \\
1960 \\
1960\end{array}$ & $\begin{array}{l}209.07040 \\
213.31537 \\
217.69096 \\
217.69096 \\
222.20257\end{array}$ & $\begin{array}{l}29,53 \\
34,20 \\
34,25 \\
36,69 \\
28,61\end{array}$ & $\begin{array}{l}426.92 \\
499.53 \\
505.34 \\
541.32 \\
426.46\end{array}$ & $\begin{array}{l}1960 \\
1960 \\
1897 \\
1960 \\
1960\end{array}$ & $\begin{array}{l}452.14186 \\
465.73936 \\
479.95959 \\
479.95959 \\
494.84118\end{array}$ & $\begin{array}{l}37.81 \\
29.40 \\
34.04 \\
28.05 \\
31.52\end{array}$ & $\begin{array}{l}804.01 \\
634.55 \\
745.76 \\
614.44 \\
701.07\end{array}$ \\
\hline $\begin{array}{l}1897 \\
1960 \\
1960 \\
1897 \\
1960 \\
1960 \\
1960 \\
1962 \\
1960 \\
1961\end{array}$ & $\begin{array}{l}226.85590 \\
226.85590 \\
231.65695 \\
236.61204 \\
236,61204 \\
241.72783 \\
247.01134 \\
249.71829 \\
252,46999 \\
255.26742\end{array}$ & $\begin{array}{l}23,82 \\
31,01 \\
12,55 \\
25,75 \\
25,75 \\
23,09 \\
25,58 \\
32,66 \\
54,35 \\
81,88\end{array}$ & $\begin{array}{l}358.71 \\
467.02 \\
190.94 \\
396.11 \\
396.03 \\
358.93 \\
402.03 \\
516.09 \\
863.57 \\
1308.28\end{array}$ & $\begin{array}{l}1897 \\
1960 \\
1960 \\
1897 \\
1960 \\
1960 \\
1897 \\
1960 \\
1960 \\
1897\end{array}$ & $\begin{array}{l}510.42578 \\
510.42578 \\
526.75838 \\
543.88761 \\
543.88761 \\
561.86616 \\
580.75111 \\
580.75111 \\
600.60442 \\
621.49345\end{array}$ & $\begin{array}{l}31.89 \\
35.73 \\
21.55 \\
26.22 \\
26.42 \\
22.52 \\
32.13 \\
29.09 \\
30.57 \\
28.25\end{array}$ & $\begin{array}{l}7.20 .46 \\
807.25 \\
494.65 \\
611.46 \\
616.20 \\
533.83 \\
774.29 \\
701.15 \\
749.14 \\
704.36\end{array}$ \\
\hline 1962 & 255,26742 & 85,93 & 1372.96 & 1.960 & 621.49345 & 33.23 & 828,44 \\
\hline $\begin{array}{l}1961 \\
1962 \\
1960 \\
1962 \\
1960 \\
1960 \\
1897 \\
1960 \\
1960\end{array}$ & $\begin{array}{l}261,00359 \\
261,00359 \\
263.94445 \\
266,93530 \\
269.97727 \\
276,21931 \\
282,68037 \\
282.68037 \\
289.37079\end{array}$ & $\begin{array}{l}89,97 \\
95,86 \\
54,82 \\
50,24 \\
33,38 \\
30,48 \\
29,00 \\
23,65 \\
23,17\end{array}$ & $\begin{array}{r}1453.59 \\
1548.61 \\
890.65 \\
820.78 \\
548.47 \\
506.56 \\
487.61 \\
397.58 \\
394.12\end{array}$ & $\begin{array}{l}1960 \\
1897 \\
1960 \\
1960 \\
1897 \\
1960 \\
1960 \\
1897 \\
1960\end{array}$ & $\begin{array}{l}643,49153 \\
666,67857 \\
666.67857 \\
691.14182 \\
716.97668 \\
716.97668 \\
744.28764 \\
773,18935 \\
773,18935\end{array}$ & $\begin{array}{l}33.91 \\
34.51 \\
36.19 \\
26.64 \\
25.58 \\
27.11 \\
32.83 \\
28.50 \\
28.57\end{array}$ & $\begin{array}{l}860.31 \\
891.02 \\
934.45 \\
700.28 \\
684.91 \\
725.90 \\
895.55 \\
792.57 \\
794.49\end{array}$ \\
\hline $\begin{array}{l}1897 \\
1960 \\
1960\end{array}$ & $\begin{array}{l}296.30159 \\
296.30159 \\
303\end{array}$ & $\begin{array}{l}26,41 \\
29: 84 \\
34: 75\end{array}$ & $\begin{array}{l}454.57 \\
513.57 \\
605.43\end{array}$ & $\begin{array}{l}1960 \\
1897 \\
1960\end{array}$ & $\begin{array}{l}803,80776 \\
836,28159 \\
836\end{array}$ & $\begin{array}{l}21.56 \\
24.02 \\
25\end{array}$ & $\begin{array}{l}611.21 \\
694.55 \\
742.74\end{array}$ \\
\hline $\begin{array}{l}1960 \\
1897 \\
1960 \\
1960\end{array}$ & $\begin{array}{l}303.48439 \\
310,93159 \\
310,93159 \\
318,65630\end{array}$ & $\begin{array}{l}34.75 \\
30,87 \\
37.82 \\
46,55\end{array}$ & $\begin{array}{l}605.43 \\
544.25 \\
666.84 \\
830.88\end{array}$ & $\begin{array}{l}1960 \\
1960 \\
1897 \\
1960\end{array}$ & $\begin{array}{l}836.28159 \\
870.76385 \\
907.92361 \\
907,42361\end{array}$ & $\begin{array}{l}25,68 \\
21.11 \\
23.39 \\
20,69\end{array}$ & $\begin{array}{l}742.74 \\
622.96 \\
704.47 \\
623.39\end{array}$ \\
\hline $\begin{array}{l}1897 \\
1960 \\
1960 \\
1897\end{array}$ & $\begin{array}{l}326,67250 \\
326.67250 \\
334.99503 \\
343.63971\end{array}$ & $\begin{array}{l}38,31 \\
38,20 \\
40,07 \\
40,32\end{array}$ & $\begin{array}{l}692.49 \\
690.44 \\
733.46 \\
747.44\end{array}$ & $\begin{array}{l}1960 \\
1897 \\
1960 \\
1960\end{array}$ & $\begin{array}{r}946,44817 \\
988.04536 \\
988,04536 \\
1032.44641\end{array}$ & $\begin{array}{l}23.89 \\
26.40 \\
27.73 \\
26.27\end{array}$ & $\begin{array}{l}734.97 \\
829.95 \\
871.68 \\
844.10\end{array}$ \\
\hline $\begin{array}{l}1960 \\
1960 \\
1897\end{array}$ & $\begin{array}{l}343.63971 \\
352.62337 \\
361.96399\end{array}$ & $\begin{array}{l}40,17 \\
43,07 \\
31,00\end{array}$ & $\begin{array}{l}744,59 \\
808,84 \\
589,78\end{array}$ & $\begin{array}{l}1897 \\
1960 \\
1960\end{array}$ & $\begin{array}{l}1079.90909 \\
1079.90909 \\
1130.72147\end{array}$ & $\begin{array}{l}27.04 \\
27.59 \\
33.19\end{array}$ & $\begin{array}{r}888.67 \\
906.76 \\
1116.21\end{array}$ \\
\hline $\begin{array}{l}1960 \\
1960 \\
1897 \\
1960\end{array}$ & $\begin{array}{l}361.96399 \\
371.68071 \\
381.79401 \\
381.79401\end{array}$ & $\begin{array}{l}19,33 \\
15: 06 \\
24,55 \\
27.79\end{array}$ & $\begin{array}{l}367.79 \\
290.30 \\
479.60 \\
542.96\end{array}$ & $\begin{array}{l}1897 \\
1960 \\
1960 \\
1897\end{array}$ & $\begin{array}{l}1185.20634 \\
1185,20634 \\
1243.72635 \\
1306.68999\end{array}$ & $\begin{array}{l}24.98 \\
23.05 \\
26.50 \\
28.12\end{array}$ & $\begin{array}{r}859.82 \\
793.71 \\
934.51 \\
1016.52\end{array}$ \\
\hline $\begin{array}{l}1960 \\
1897 \\
1960\end{array}$ & $\begin{array}{l}392.32576 \\
403.29938 \\
403.29938\end{array}$ & $\begin{array}{l}26,89 \\
26,97 \\
18,75\end{array}$ & $\begin{array}{l}532.68 \\
541.71 \\
376.63\end{array}$ & $\begin{array}{l}1960 \\
1960 \\
1897\end{array}$ & $\begin{array}{l}1306.68999 \\
1374.55884 \\
1447.85594\end{array}$ & $\begin{array}{l}24.45 \\
24.40 \\
29.40\end{array}$ & $\begin{array}{r}883.88 \\
904.47 \\
1118,55\end{array}$ \\
\hline
\end{tabular}




\begin{tabular}{|c|c|c|c|}
\hline ID & ENERGY & SIGMA & SIGMA \\
\hline $\begin{array}{l}1960 \\
1960\end{array}$ & $\begin{array}{c}\text { (EV) } \\
1447.85594 \\
1527.17604\end{array}$ & $\begin{array}{r}\text { (BARNS) } \\
23.39 \\
29.64\end{array}$ & $\begin{array}{r}\text { ROOT E } \\
889.88 \\
1.158,12\end{array}$ \\
\hline $\begin{array}{l}1897 \\
1960 \\
1960\end{array}$ & $\begin{array}{l}1613,19753 \\
1613.19753 \\
1706,69713\end{array}$ & $\begin{array}{l}28,39 \\
26,18 \\
19,97\end{array}$ & $\begin{array}{r}1140,15 \\
1051.49 \\
825,08\end{array}$ \\
\hline $\begin{array}{l}1897 \\
1960 \\
1960\end{array}$ & $\begin{array}{l}1808,56746 \\
1808,56746 \\
1919,83838\end{array}$ & $\begin{array}{l}26,23 \\
21: 65 \\
23,18\end{array}$ & $\begin{array}{r}1115.54 \\
920.52 \\
1015.47\end{array}$ \\
\hline $\begin{array}{l}1897 \\
1960\end{array}$ & $\begin{array}{l}20.41,70313 \\
2041.70313\end{array}$ & $\begin{array}{l}22,57 \\
24,11\end{array}$ & $\begin{array}{l}1019.62 \\
1089.45\end{array}$ \\
\hline $\begin{array}{l}1960 \\
1897 \\
1960\end{array}$ & $\begin{array}{l}2175,0.5045 \\
2323,00443 \\
2323,00443\end{array}$ & $\begin{array}{l}26,95 \\
28,55 \\
22,71\end{array}$ & $\begin{array}{l}1257,15 \\
1375,84 \\
1094,35\end{array}$ \\
\hline $\begin{array}{l}1960 \\
1897\end{array}$ & $\begin{array}{l}2485,9.7382 \\
2666, .71426\end{array}$ & 27.23 & $\begin{array}{l}1357.61 \\
1344.52\end{array}$ \\
\hline $\begin{array}{l}1960 \\
19.60 \\
1897\end{array}$ & $\begin{array}{l}2666,71426 \\
2867,90671 \\
3092,75739\end{array}$ & $\begin{array}{l}17: 57 \\
20,35 \\
18,01\end{array}$ & $\begin{array}{r}907.57 \\
1089.65 \\
1001.43\end{array}$ \\
\hline $\begin{array}{l}1960 \\
1960\end{array}$ & $\begin{array}{l}3092,75739 \\
3345,12637\end{array}$ & $\begin{array}{l}23,62 \\
16,77\end{array}$ & $\begin{array}{r}1313.67 \\
970 . .20\end{array}$ \\
\hline 1897 & $3629,6,9443$ & 24,26 & 1461.68 \\
\hline $\begin{array}{l}1960 \\
1960\end{array}$ & $\begin{array}{l}3629.69443 \\
3952.18146\end{array}$ & $\begin{array}{l}24.62 \\
20,37 \\
26.69\end{array}$ & $\begin{array}{l}1483,14 \\
1280,31 \\
1754.29\end{array}$ \\
\hline $\begin{array}{l}1897 \\
1960\end{array}$ & $\begin{array}{l}4319.63635 \\
4319.63635\end{array}$ & $\begin{array}{l}26.69 \\
20.60\end{array}$ & $\begin{array}{l}1754.29 \\
1354.02\end{array}$ \\
\hline $\begin{array}{l}1960 \\
1960 \\
1960\end{array}$ & $\begin{array}{l}4319.63635 \\
4740.82538 \\
5226.75995\end{array}$ & $\begin{array}{l}20,60 \\
21,36 \\
15,02\end{array}$ & $\begin{array}{l}1354.02 \\
1470.49 \\
1085.68\end{array}$ \\
\hline $\begin{array}{l}1960 \\
1960 \\
1960\end{array}$ & $\begin{array}{l}5791,42377 \\
6452,79010 \\
7234,26984\end{array}$ & $\begin{array}{l}16,81 \\
22,19 \\
19,73\end{array}$ & $\begin{array}{l}1279.52 \\
1782.86 \\
1677.76\end{array}$ \\
\hline $\begin{array}{l}1960 \\
1960\end{array}$ & $\begin{array}{l}8166,81250 \\
9292,01770\end{array}$ & $\begin{array}{l}22,67 \\
22,09\end{array}$ & $\begin{array}{l}2048.32 \\
2129.44\end{array}$ \\
\hline
\end{tabular}

